\title{
Southern Fine Particulate Monitoring Project
}

\author{
Final Technical Report \\ Reporting Period: October 1, 2000 - September 30, 2002 \\ Principal Author: Ashley D. Williamson \\ Issued: November, 2005 \\ DOE Cooperative Agreement No. DE-FC26-00NT40770
}

\author{
Submitted to \\ U.S. DEPARTMENT OF ENERGY \\ National Energy Technology Laboratory \\ P. O. Box 10940 \\ 626 Cochrans Mill Road \\ Pittsburgh, PA 15236-0940 \\ Attn: William Aljoe \\ Prepared by \\ SOUTHERN RESEARCH INSTITUTE \\ 2000 Ninth Avenue South \\ P. O. Box 55305 \\ Birmingham, AL 35255-5305 \\ Principal Investigator: Ashley D. Williamson
}




\title{
Disclaimer
}

This report was prepared as an account of work sponsored by an agency of the United States Government. Neither the United States Government or any agency thereof, nor any of their employees, makes any warranty, express or implied, or assumes any legal liability or responsibility for the accuracy, completeness, or usefulness of any information, apparatus, product, or process disclosed, or represents that its use would not infringe privately owned rights. Reference herein to any specific commercial product, process, or service by trade name, trademark, manufacturer, or otherwise does not necessarily constitute or imply its endorsement, recommendation, or favoring by the United States Government or any agency thereof. The views and opinions of authors expressed herein do not necessarily state or reflect those of the United States Government or any agency thereof.

\begin{abstract}
This final project report presents experimental details, results and analysis of continuous onsite ambient fine particulate data at the North Birmingham sampling site during the October, 2001 September, 2002 study period. he host site for these measurement activities is the North Birmingham PM monitoring station by the Jefferson County Health Department in Birmingham, AL.The continuous data include $\mathrm{PM}_{2.5}$ mass concentrations measured by TEOM, particle sulfate using the R\&P 8400S monitor, particle size distributions measured by SMPS and APS monitors, and $\mathrm{PM}_{2.5}$ light scattering extinction coefficient as measured by nephelometer. During the course of the project, measurement intercomparison data were developed for these instruments and several complementary measurements at the site. The report details the instrument set and operating procedures and describes the resulting data. Report subsections present an overview summary of the data, followed by detailed description of the systematic time behavior of $\mathrm{PM}_{2.5}$ and other specific particulate size fractions. Specific subsections are included for particle size distribution, light scattering, and particle sulfate data. The final subsection addresses application of the measurements to the practical questions of fine PM generation and transport, source attribution, and $\mathrm{PM}_{2.5}$ management strategies.
\end{abstract}




\section{Table of Contents}

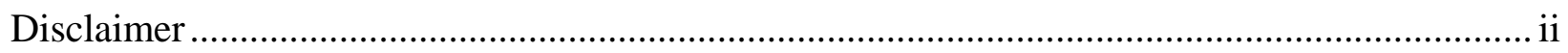

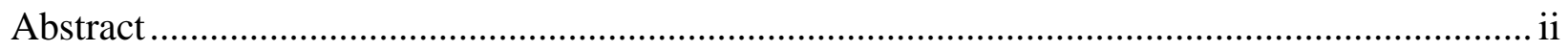

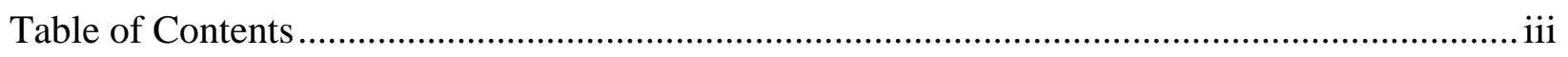

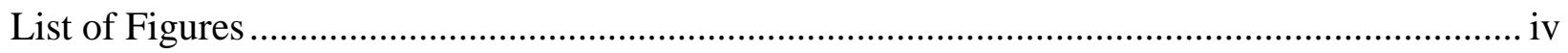

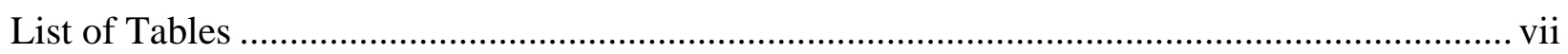

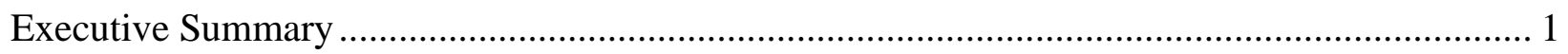

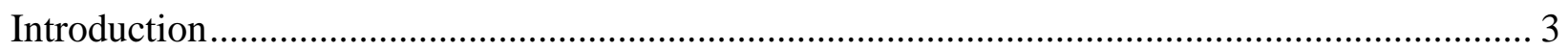

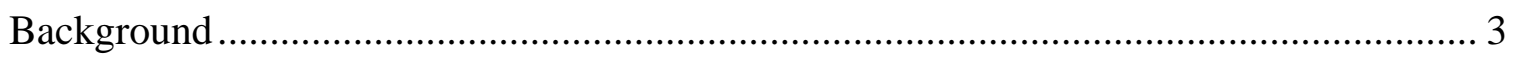

Characteristics of North Birmingham study location .................................................... 5

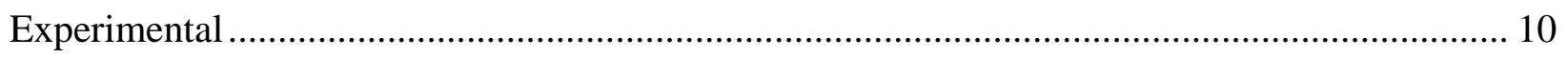

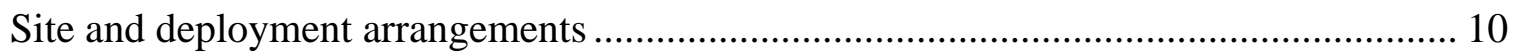

Instrument Configuration and Operating Procedures .............................................. 11

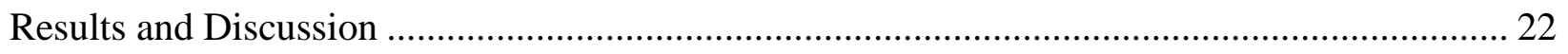

Summary of Particulate Measurement Data ........................................................... 22

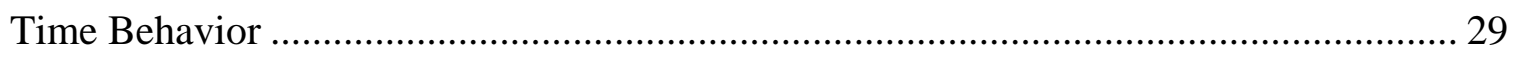

Comparative Measurements of Particulate Properties and Composition ........................ 55

Application to Source Attribution, PM Transport, and PM P.5 $_{2.5}$ Management Strategy ...... 91

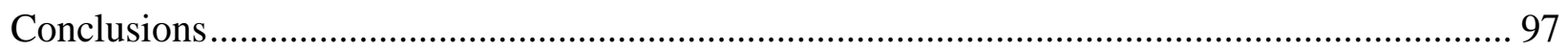




\section{List of Figures}

Figure 1. Map of Birmingham/Jefferson County showing location of North Birmingham Site (NOBI) and other County Ambient Air Quality Monitoring Stations.....

Figure 2. Map of Birmingham Metropolitan Area showing N. Birmingham site and sources outside of the city limits.

Figure 3. Expanded map of Downtown Birmingham showing monitoring site and local industrial sources.

Figure 4. Photograph of the North Birmingham Monitoring site managed and maintained by the Jefferson County Health Department.....

Figure 5. Rear view of the North Birmingham Monitoring site before installation of the sampling shelter for this project, showing the SEARCH shelter and towers. ............ 11

Figure 6. Floor plan of sampling shelter showing location of sampler instrument racks. ......... 12

Figure 7. Sampling shelter rooftop view showing sampler inlets ...................................... 13

Figure 8. Sampler racks containing R\&P 8400S sulfate monitor (left) and TSI 3321/3496 particle sizing instrument package (right) as deployed inside shelter.

Figure 9. Sampler rack containing TEOM and Nephelometer in left corner beside rack and gas cylinders for R\&P 8400S sulfate monitor. 15

Figure 10. Flow diagram of TEOM 1400ab unit with SES as used in project.......................... 16

Figure 11. Radiance 904 Nephelometer and “smart heater” relative humidity controller........... 17

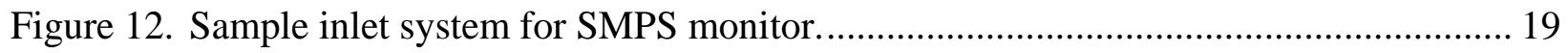

Figure 13. Daily average TEOM $\mathrm{PM}_{2.5}$ concentration over the project period, with corresponding AIRS FRM PM 2.5 data.

Figure 14. Comparison of monthly average $\mathrm{PM}_{2.5}$ concentration and 8400 S sulfate monitor data over the project period. 25

Figure 15. Comparison of monthly average $\mathrm{PM}_{2.5}, \mathrm{PM}_{10}$, and nephelometer data over the project period. 26

Figure 16. Composite monthly average particle size distribution for June, 2002 as measured by SMPS and APS.

Figure 17. Hourly average TEOM $\mathrm{PM}_{2.5}$ concentration and wind speed over four-month periods: (A) 7/1/01 - 10/31/01 summer; (B) 12/15/01 - 4/15/02 winter. 31

Figure 18. Hourly average TEOM $\mathrm{PM}_{2.5}$ concentration, wind speed and direction over two week period beginning 8/19/01. 33

Figure 19. Hourly average TEOM PM 2.5 concentration, wind speed and direction over two week period beginning 1/1/02. 34

Figure 20. PM data from several instruments for 72 hour period beginning noon, 8/23/01....... 36 
Figure 21. Real-time PM instrumental data for 48 hour period beginning noon, 1/11/02: (A) minute and hour average data; (B) TEOM, 8400S, and wind data.

Figure 22. Monthly average project period TEOM PM P.. $_{\text {in }}$ context of 2001-04 AIRS $\mathrm{PM}_{2.5}$, $\mathrm{PM}_{10}$, and PMc data: (A) by month; (B) by month of year. 39

Figure 23. Day of week average SRI TEOM PM 2.5 with AIRS $\mathrm{PM}_{2.5}, \mathrm{PM}_{10}$, and PMc data: (A) midnight to midnight; (B) starting at 5:00AM each day.

Figure 24. Day of week relative average $\mathrm{PM}_{2.5}, \mathrm{PM}_{10}$, and $\mathrm{PMc}$ data starting at 5:00AM each day.

Figure 25. Day of week relative average data from nephelometer and 8400S monitor.............. 44

Figure 26. Day of week relative average submicron size fractions from SMPS....................... 44

Figure 27. Day of week relative average APS 3321 size fractions (after March, 2002)............. 45

Figure 28. Day of week relative average concentrations from ARA gas monitor data. ............. 46

Figure 29. Hour of day average $\mathrm{PM}_{2.5}, \mathrm{PM}_{10}$, and PMc data from several instruments: (A) in mass concentration units; (B) relative to average for each instrument..................... 47

Figure 30. Hour of day relative average data from nephelometer and 8400S monitor............... 49

Figure 31. Hour of day relative average submicron size fractions from SMPS........................ 50

Figure 32. Hour of day relative average APS 3321 size fractions (after March, 2002)............. 51

Figure 33. Hour of day relative average concentrations from ARA gas monitor data................ 52

Figure 34. Hour of day ARA NO concentration average overlay for each day of the week...... 52

Figure 35. Hour of day $\mathrm{PM}_{10}$ and PMc concentration average overlay by day of the week........ 53

Figure 36. Hour of day $\mathrm{PM}_{2.5}$ and sulfate average overlay by day of the week........................ 54

Figure 37. Hour of day $\mathrm{SO}_{2}$ average overlay for ARA and 8400S monitor data....................... 55

Figure 38. Daily average $\mathrm{PM}_{2.5}$ concentration as measured by SRI and JCHD TEOMs and JCHD FRM. Ratios of TEOM averages to FRM are also shown..................................... 56

Figure 39. Daily average $\mathrm{PM}_{2.5}$ concentrations from Figure 38 with outliers deleted. Ratios of corresponding TEOM and FRM daily and monthly averages are also shown on expanded scale.

Figure 40. Daily average $\mathrm{PM}_{2.5}$ concentrations as measured by SRI and JCHD TEOMs. Ratios of daily averages for corresponding hours are also shown on expanded scale.............. 58

Figure 41. Comparison of daily average $\mathrm{PM}_{2.5}$ TEOM concentrations with JCHD FRM over the project period. 60

Figure 42. Comparison of daily average $\mathrm{PM}_{2.5}$ concentrations for corresponding hours measured by SRI and JCHD TEOMs over the project period.

Figure 43. Comparison of corresponding hourly average $\mathrm{PM}_{2.5}$ concentrations measured by SRI and JCHD TEOMs. Outliers shown were eliminated for fit. 
Figure 44. Daily average integrated SMPS mass plotted with $\mathrm{PM}_{2.5}$ concentration from JCHD FRM. Ratios of SMPS total and $\mathrm{PM}_{100}$ mass averages to SRI TEOM PM 2.5 are shown on second scale.

Figure 45. Daily average integrated SMPS number plotted with $\mathrm{PM}_{2.5}$ concentration from JCHD FRM. Ratios of SMPS total and N30 number averages to SRI TEOM PM 2.5 are shown on second scale.

Figure 46. Comparison of daily average integrated SMPS mass and number concentrations to SRI TEOM PM 2.5 over the year July, 2001 - June, 2002.

Figure 47. Comparison of daily average integrated SMPS size fractions to SRI TEOM PM 2.5 over the year July, 2001 - June, 2002.

Figure 48. Comparison of hourly average integrated SMPS mass and number concentrations to SRI TEOM PM 2.5 over the year July, 2001 - June, 2002. 66

Figure 49. Comparison of hourly average integrated SMPS size fractions to SRI TEOM PM 2.5 over the year July, 2001 - June, 2002.

Figure 50. Comparison of hourly average SMPS number concentrations in lowest (21 nm midpoint) channel to $\mathrm{PM}_{2.5}$ measured by SRI TEOM.

Figure 51. Monthly average SMPS concentrations and indicators of particle size for the project period. 68

Figure 52. Average SMPS particle size distribution for project months in differential concentration format: (A) number weighted, (B) mass weighted.

Figure 53. Average SMPS particle size distribution for project months in cumulative percent format: (A) number weighted, (B) mass weighted. 70

Figure 54. Hour of day average SMPS concentrations and indicators of particle size for the project period beginning July, 2001.

Figure 55. Hour of day average SMPS particle size distribution after July 1, 2001 in differential concentration format: (A) number weighted, (B) mass weighted.....

Figure 56. Hour of day average SMPS particle size distribution after July 1, 2001 in cumulative percent format: (A) number weighted, (B) mass weighted. 73

Figure 57. Hour of day average SMPS concentrations and indicators of particle size for August $24,2001$.

Figure 58. Hour of day average SMPS differential number weighted size distribution for 8/24/01: (A) hours before noon (B) hours after noon.

Figure 59. Expanded scale of SMPS differential number plot in Figure 58B, showing residual size distribution envelope. 76

Figure 60. Hour of day average mass weighted SMPS particle size distribution for 8/24/01.... 77

Figure 61. Daily average integrated APS mass below $2.5 \mu \mathrm{m}$ plotted with $\mathrm{PM}_{2.5}$ concentration from JCHD FRM. Ratios of APS mass averages to PM $_{2.5}$ from FRM and SRI TEOM are shown on second scale. 
Figure 62. Daily average integrated APS mass between 2.5 and $10 \mu \mathrm{m}$ plotted with PMc calculated from JCHD TEOMs. Ratios of APS mass averages to PMc and SRI TEOM $\mathrm{PM}_{2.5}$ are shown on second scale.

Figure 63. Comparison of daily average APS integrated mass concentrations $<2.5 \mu \mathrm{m}$ to $\mathrm{PM}_{2.5}$ from SRI TEOM before and after APS upgrade.

Figure 64. Comparison of daily average integrated PMc from APS before and after upgrade to PMc concentrations calculated from JCHD TEOMs.

Figure 65. Average monthly differential mass weighted particle size distribution for APS instruments: (A) APS 3320, (B) upgraded APS 3321.

Figure 66. Average monthly cumulative percent mass weighted particle size distribution for APS instruments: (A) APS 3320, (B) upgraded APS 3321.

Figure 67. Normalized monthly differential mass weighted particle size distribution for APS instruments: (A) APS 3320, (B) upgraded APS 3321.

Figure 68. Daily average Nephelometer Bsc values compared to $\mathrm{PM}_{2.5}$ mass concentration from JCHD FRM. Ratios of Nephelometer Bsc averages to $\mathrm{PM}_{2.5}$ from FRM and SRI TEOM are shown on second scale.

Figure 69. Scatter plot of daily average Nephelometer Bsc values compared to SRI TEOM and FRM PM 2.5 mass concentration. 88

Figure 70. Daily average particle sulfate mass concentration measurements by SRI $8400 \mathrm{~S}$ monitor compared to ARA PCM sulfate and SRI TEOM. Ratios of 8400S sulfate to PCM sulfate and to TEOM mass are shown on second scale.

Figure 71. Scatter plot of daily average 8400 S sulfate mass concentration compared to ARA PCM sulfate over project period.

Figure 72. Scatter plot of daily average 8400 S sulfate mass concentration compared to SRI PM 2.5 TEOM over project period.

Figure 73. Ten minute average wind direction values (binned in 10 degree increments) corresponding to 8400S data from July 2001 through January 2002.

Figure 74. Three individual episodes during July 10 - 16, 2001 analyzed for source identification utility.

Figure 75. SMPS differential number size distributions for two individual events on July 15, 2001.

\section{List of Tables}

Table 1. Monthly average particulate measurement data during project period (all values in $\mu \mathrm{g} / \mathrm{m}^{3}$ unless otherwise indicated). 


\section{Executive Summary}

This final technical report summarizes findings of the "Southern Fine Particulate Monitoring Project”, funded by the U.S. Department of Energy's National Energy Technology Laboratory under DOE Cooperative Agreement No. DE-FC26-00NT40770 to Southern Research Institute (SRI). In this two year project SRI conducted detailed studies of ambient fine particulate matter in the Birmingham, AL metropolitan area. Project objectives included: augmented measurements at the established site; detailed database of near-continuous fine particulate measurements; application to source attribution, time/transport properties, management strategies for $\mathrm{PM}_{2.5}$; measurement method validation/comparison.

The host site for these measurement activities is the North Birmingham PM monitoring station maintained and operated by the Jefferson County Health Department (JCHD) - the local regulatory agency for NAAQS matters - in Birmingham, AL. The other major benefit of the North Birmingham site for this proposed work is the opportunity for collaboration with the Southeastern Aerosol Research and Characterization (SEARCH) project, managed by Atmospheric Research \& Analysis, Inc. (ARA) for the Electric Power Research Institute (EPRI), with the Southern Company as a major sponsoring organization. The North Birmingham site is also one of eight sites in the regional monitoring network of the Southeastern Aerosol Research and Characterization (SEARCH) project, managed by Atmospheric Research \& Analysis, Inc. (ARA) for the Electric Power Research Institute (EPRI).

The project measurement suite consists of measurements made by five instruments in the new sampling shelter installed at the site. These include: an R\&P 1400ab Tapered Element Oscillating Microbalance (TEOM) particulate mass monitor with $\mathrm{PM}_{2.5}$ Sharp Cut Cyclone; Radiance 904 integrating nephelometer, an R\&P 8400S particulate sulfate monitor; and a particle sizing instrument package. The sizing instruments are a Model 3496 Scanning Mobility Particle Sizer (SMPS), and Model 3320 Aerodynamic Particle Sizer (APS), both products of TSI, Inc.. The APS was upgraded to a Model 3321 configuration during the project. During the course of the project, measurement intercomparison data were developed for these instruments and several complementary measurements at the site.

The entire measurement suite was installed in the project shelter in June, 2001, and became fully operational in July; preliminary measurements had been made with most of the instruments in March. The planned operation for the project was to continue until August, 2002, though most instruments were left running with reduced operator attention through the end of the project period in September, 2002, and the data were used, where suitable, in the analyses.

Several relevant findings of this project regarding local fine particulate are summarized below.

The North Birmingham site experiences ambient $\mathrm{PM}_{2.5}$ concentrations in excess of the Ambient Air Quality Standard. While no day in our study exceeded the $65 \mu \mathrm{g} / \mathrm{m}^{3}$ daily average standard for $\mathrm{PM}_{2.5}, 60 \%$ of the days exceeded the $15 \mu \mathrm{g} / \mathrm{m}^{3}$ annual standard, $39 \%$ were over $20 \mu \mathrm{g} / \mathrm{m}^{3}$, and $15 \%$ over $30 \mu \mathrm{g} / \mathrm{m}^{3}$, resulting in a (non-calendar year) twelve month average of $18.5 \mu \mathrm{g} / \mathrm{m}^{3}$, consistent with the standard exceedences every year since the beginning of the project.

$\mathrm{PM}_{2.5}$ concentrations at the site follow a seasonal time course, with elevated levels in the summer months and lower concentrations in the December - February periods. The short-term time course of the $\mathrm{PM}_{2.5}$ concentrations likewise has characteristic summer and winter patterns, related to the seasonal meterological patterns. The summer period is characterized by recurring 
multiday periods having elevated $\mathrm{PM}_{2.5}$ in conjunction with lower mean daily wind speed and very low overnight wind speed. These occur most notably as clear, sunny, stagnant periods under the influence of stable high pressure cells which also lead to episodes of elevated ambient ozone. During these periods particulate levels follow a prototypical daily pattern, with a characteristic morning peak shortly after sunrise, falling quickly to fairly stable low levels during the daylight hours, then rising gradually to elevated levels overnight, then rapidly into the next morning peak. Winter multiday episodes are driven by a different regional weather pattern: they are less frequent, shorter in duration, and generally lower in concentration. While the day/night pattern is always evident, the morning peak behavior is less regular and may be absent. These time patterns are described in detail in the full report.

During the stagnant episode periods with highest particulate concentrations, the impact of local sources is proportionally very high. This effect is seen in several study results, including:

- a significant weekday/weekend difference, which varies with pollutant and particle size range. The greatest variation is seen in $\mathrm{PMc}$, NO and NOy , and the ultrafine particle size range $(<100 \mathrm{~nm})$.

- The morning peak is reduced in size on weekends as source emissions decrease. For the most reactive species above, the peak disappears entirely on Sunday mornings.

- recurring patterns of submicron particle size distribution, as a stable aged urban/regional aerosol with peak particle size around 200nm mixes with a changing amounts of fresh locally generated aerosol. The local particle mix is numerically dominated by a particle mode around 50nm typical peak diameter, but contains frequent bursts of micron-range and coarse particles in local source plumes.

$\mathrm{SO}_{2}$ has a different time pattern from the other pollutants, and is less in sync with daily atmospheric mixing patterns. It is apparently much more affected by local source emissions patterns, and accordingly is vary useful as a tracer for these sources, which include several nearby industrial sources as well as the coal-fired utilities near the metropolitan area.

Other sources can be identified by combustion tracers (CO, NO/NOy) as well as characteristic particle size distributions.

Source attribution will likely be an important part of developing management strategy for $\mathrm{PM}_{2.5}$. This is especially true of the North Birmingham site, which among the local monitoring stations has the highest average concentrations. The site is clearly impacted by nearby sources, but the highest source contributions occur during stagnant periods and are not directly tied to the responsible sources by measured wind directions. 


\section{Introduction}

This final technical report summarizes findings of the "Southern Fine Particulate Monitoring Project”, funded by the U.S. Department of Energy's National Energy Technology Laboratory under DOE Cooperative Agreement No. DE-FC26-00NT40770 to Southern Research Institute (SRI). In this two year project SRI conducted detailed studies of ambient fine particulate matter in the Birmingham, AL metropolitan area. As addressed in this report, project objectives included:

- $\quad$ Augment existing measurements of primary and secondary aerosols at an established urban southeastern monitoring site

- $\quad$ Make a detailed database of near-continuous measurements of the time variation of fine particulate mass, composition, and key properties (including particle size distribution)

- $\quad$ Apply the measurements to source attribution, time/transport properties of fine PM, and implications for management strategies for $\mathrm{PM}_{2.5}$

- $\quad$ Validate and compare key measurement methods used in this study for applicability within other $\mathrm{PM}_{2.5}$ research by DOE-FE, EPA, NARSTO, and others.

\section{Background}

On July 17, 1997, the U.S. Environmental Protection Agency (EPA) revised the National Ambient Air Quality Standards (NAAQS) to address ambient air concentrations of particulate matter with an aerodynamic diameter of 2.5 micrometers or less $\left(\mathrm{PM}_{2.5}\right)$. The new $\mathrm{PM}_{2.5}$ standard (EPA 1997) established a 24-hour average concentration limit of $65 \mu \mathrm{g} / \mathrm{m}^{3}$ and an annual mean concentration limit of $15 \mu \mathrm{g} / \mathrm{m}^{3}$ to protect human health from both chronic and acute effects associated with the respiration of fine particulate matter (PM). The previous standard, which was in effect retained, regulated $\mathrm{PM}_{10}$ (particulate matter with an aerodynamic diameter of 10 micrometers or less), for which an ambient sampling network of some 1400 sites exists. The new standard would be monitored by a new network of up to 1500 monitoring stations for $\mathrm{PM}_{2.5}$, using a new Federal Reference Method (FRM) sampler developed by the EPA. The new network was necessitated by the near absence of systematic measurements of $\mathrm{PM}_{2.5}$ in the U.S. in previous years. The data compiled for the 1996 Particulate Matter Criteria Document (EPA 1996a) relied primarily on less than 50 urban sites and 38 non-urban IMPROVE (Interagency Monitoring of Protected Visual Environments) sites, located in national park sites (Malm, et al., 1994). Unfortunately, the spatial coverage of this historical data set left much to be desired.

The general picture deduced from the IMPROVE network data in the 1996 Criteria Document (EPA 1996a) is a general division of the country around the Mississippi River into two (eastern and western) characteristic regions of PM climatology. The non-urban eastern zone was typically higher in $\mathrm{PM}_{2.5}$ mass than the western zone, with highest levels in late summer, and annual averages close to the $15 \mu \mathrm{g} / \mathrm{m}^{3}$ annual NAAQS. Chemical speciation measurements suggested a fairly widespread dominance of sulfate aerosols (as ammonium sulfate) and organic carbon in the non-urban east, compared to a more variable aerosol in the west, with less sulfate but higher in nitrate as well as high in organic carbon. Most of the sulfate and some unknown portion of the carbon $\mathrm{PM}_{2.5}$ mass components were secondary particulate matter, formed by atmospheric reactions of gaseous precursors. Looking within the non-urban eastern region, the 
IMPROVE data suggested local maxima in $\mathrm{PM}_{2.5}$ mass, sulfate, and organics in the southeastern US. This is consistent with locally higher visibility attenuation that is also noted in this network, and which is modeled to be predominantly due to particulate sulfate constituents. The sulfate wet deposition flux follows this same geographic pattern, even though $\mathrm{SO}_{2}$ emissions are much lower in the southeast than in the Ohio River Valley area immediately to the north, and average ambient $\mathrm{SO}_{2}$ concentrations in the (southeastern) EPA Region 4 were the lowest in the eastern U.S. (EPA 1998). Clearly other factors beside precursor emissions or concentrations are active in this region.

Ironically, the historical distribution of study information left the deep South as the least-studied region likely to be highly impacted by the new $\mathrm{PM}_{2.5}$ standard. If the southeastern regional background was indeed as high as suggested by the IMPROVE results quoted above and by contemporary TVA analyses of historical data, many southeastern rural counties would be in marginal compliance with the standard before the effects of any local sources were added, leaving urban areas intractably in nonattainment status. Meanwhile, the effects of several important aspects of the regional climate were not well known, including the effects of the distinctive biogenic organic emissions, the higher humidity and temperatures characteristic of summertime episodes, and the distinctive meteorology which is highly affected by the Gulf of Mexico. Likewise, the effects of the somewhat distinctive emissions source patterns in the region were not well characterized. Compared to the Ohio River Valley area, emissions of important precursor gases (especially $\mathrm{SO}_{2}, \mathrm{NOx}$ and $\mathrm{NH}_{3}$ ) are much lower per unit area, and local urban sources tend to be less dense (EPA, 1998). In the major urban areas (Atlanta and Birmingham) mobile source emissions are influenced by high vehicle mile usage, while the industrial emissions of these two metropolitan areas are quite different from one another and from the industrial centers along the Gulf Coast (Houston, Mobile, and the lower Mississippi delta area). There was a clear need for further characterization of the deep South PM, both regional and urban, in conjunction with similar characterization at other major east central and northeastern areas.

In this time period several federally funded monitoring activities were established to mitigate the knowledge gaps regarding the sources and characteristics of ambient $\mathrm{PM}_{2.5}$ in several US regions. Most of this effort was assigned to the EPA; FY98 Congressional Appropriations also called for the U.S. Department of Energy's Office of Fossil Energy (DOE-FE) to initiate a research program to address these technical and scientific issues from the standpoint of the impact of the new standard on coal-based power systems. The largest component of DOE-FE's ambient PM2.5 monitoring activities was focused in the Ohio River Valley area, containing the largest population of coal-fired utility power units. This effort includes the Upper Ohio River Valley Project (UORVP), involving the installation and operation of ambient fine particulate monitoring sites in an area encompassing eastern Ohio, northwestern West Virginia, and western Pennsylvania, supplemented by the Pittsburgh Air Quality Study, and by complementary efforts at the DOE National Energy Technology Laboratory (NETL) in the Pittsburgh, PA area.

The Southern Fine Particulate Monitoring Project represents a complementary monitoring project to the DOE activities in the Upper Ohio River Valley area. This project applies similar goals and study methodology to a geographic focus in the southeast centered around Birmingham, AL. There are several expected benefits from the site of this project. First, the similarities and differences between the two urban centers allow a study comparison to highlight the results of different climatological settings and perhaps the differing effects of possible 
control strategies. The metropolitan areas have a similar historical source profile given the background of steel-related industries in the areas. They share a similar ridge-and-valley topography which affects air movement and pollution transport as well as meteorology. They both appear to be impacted by strong regional $\mathrm{PM}_{2.5}$ components, but also lie within the plumes of local and subregional fossil-fired utility and other industrial sources. On the other hand, the upwind source strength of important $\mathrm{PM}_{2.5}$ gaseous precursors (except for biogenic organic species) is stronger near Pittsburgh, while the regional $\mathrm{PM}_{2.5}$ background appears to be stronger near Birmingham. The mechanisms behind this difference were expected to be enlightening. Second, the southeastern study was opportune in that DOE funds could be highly leveraged by collaboration with ongoing measurement activities in the area (the EPRI-Southern Company SEARCH program) and with anticipated EPA-funded network activities by state and local agencies.

\section{Characteristics of North Birmingham study location}

The host site for SRI's measurement activities is the North Birmingham $\mathrm{PM}_{10}$ monitoring station maintained and operated by the Jefferson County Health Department (JCHD) - the local regulatory agency for NAAQS matters - in Birmingham, AL. Figure 1 contains a map of the Birmingham / Jefferson County area showing the locations of air quality monitoring stations. The North Birmingham site (shown as NOBI in the figure) is a key location in the state and region from the standpoints of historical measurement data as well as ongoing measurement data in the EPA NAAQS regulatory program. The site has been an EPA National Air Monitoring Station (NAMS) for $\mathrm{PM}_{10}$ for several years and is a NAMS Core $\mathrm{PM}_{2.5}$ mass monitoring station in the new EPA PM 2.5 network, as well as one of some 50 EPA PM$_{2.5}$ Speciation Trends Network (STN) sites across the country for trends monitoring. The county has for several years maintained continuous (TEOM - Tapered Element Oscillating Microbalance) and periodic FRM $\mathrm{PM}_{10}$ monitors, and around the beginning of this project added $\mathrm{PM}_{2.5}$ TEOM and FRM Sequential $\mathrm{PM}_{2.5}$ monitors, the Andersen RAAS speciation sampler, and an ozone monitor. The Birmingham Metropolitan Statistical Area (MSA) is also monitored by several other Jefferson County $\mathrm{PM}_{2.5}$ sites (4-6 other sites with FRMs, one of which is a second EPA speciation site). The County has historically encouraged collaborative research studies at this and other locations. The platform has adequate space and infrastructure to support method intercomparison studies, several of which have been conducted in the past few years. Thus, the opportunity for collaboration with the County was a major factor in the siting decision for this project.

The other major benefit of the North Birmingham site for this proposed work is the opportunity for collaboration with the Southeastern Aerosol Research and Characterization (SEARCH) project, managed by Atmospheric Research \& Analysis, Inc. (ARA) for the Electric Power Research Institute (EPRI), with the Southern Company as a major sponsoring organization. The North Birmingham site is one of eight sites in the SEARCH regional monitoring network, organized into four urban-rural pairs in GA, AL, FL, and MS. Since late 1998 the site has had an operating Particle Composition Monitor (PCM) designed by ARA, Inc., the operating contractor for the SEARCH program. The PCM is a batch speciation sampler which provides daily average (24 hr) filter samples of PM2.5 for analysis of major ions, trace metals, OC, and EC. Daily samples were taken and analyzed during 1999; during the period of this project, samples were generally taken on a 1 / three day schedule, supplemented by periods of daily data each January and July. Starting in mid-2000, the SEARCH program steadily added new measurements at the site. These include standard surface meteorological data from a $10 \mathrm{~m}$ tower, continuous gaseous 
pollutant measurements (CO, SO2, reactive nitrogen species), and continuous measurements of several PM components. These include particulate carbon (with an R\&P 5400 carbon analyzer) and black carbon (by aethelometer), and major secondary ions using a proprietary $\mathrm{NH}_{4} / \mathrm{NO}_{3} / \mathrm{SO}_{4}$ analyzer system developed by ARA. Identical samples are taken at the rural Centerville, AL SEARCH site. This pairing allows measurement comparison of local (urban) and regional (rural) $\mathrm{PM}_{2.5}$ and precursors. Like the Jefferson County Health Department, the SEARCH program was willing to collaborate with the proposed project, including coordinated measurements and data sharing.

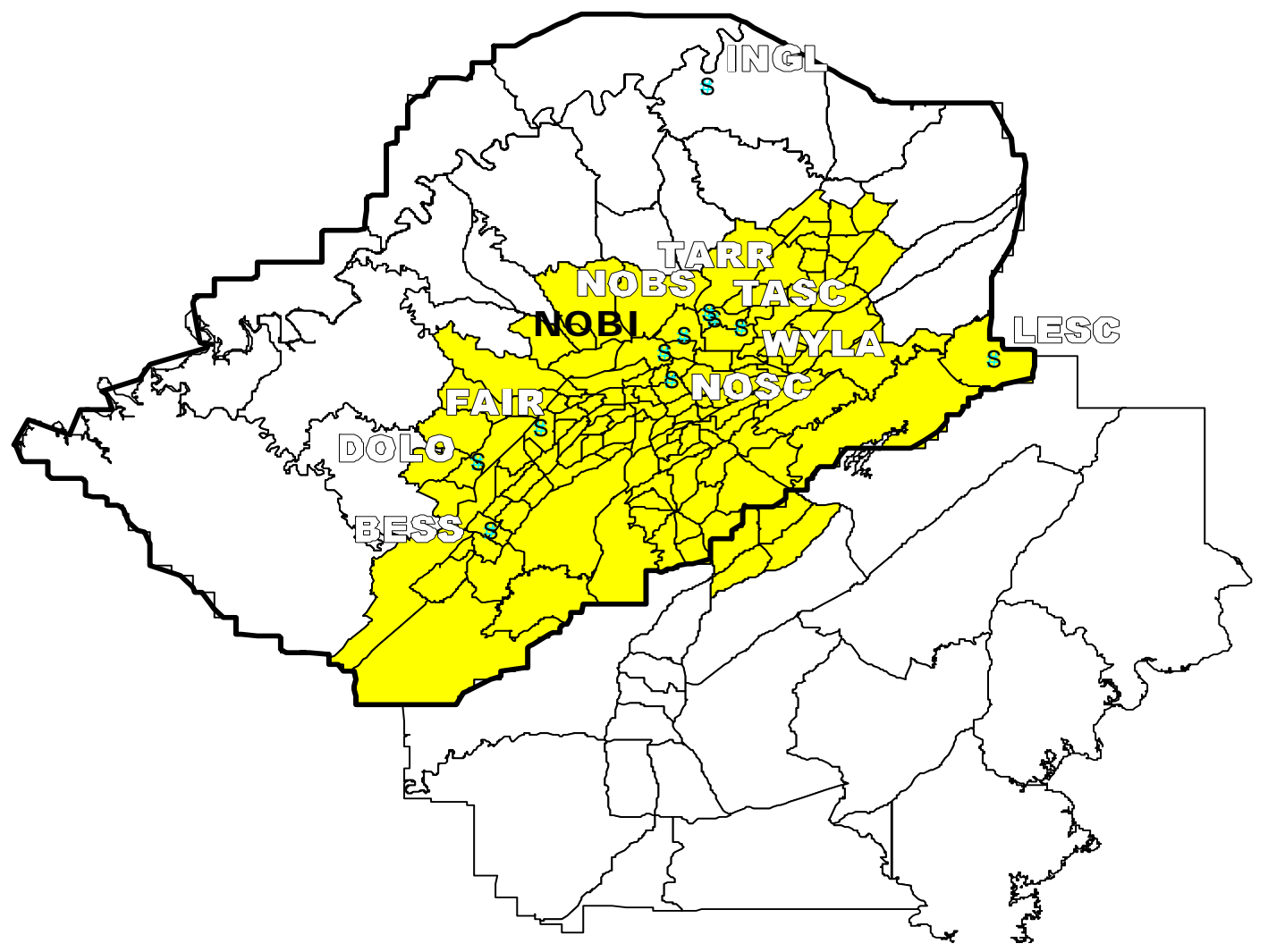

Figure 1. Map of Birmingham/Jefferson County showing location of North Birmingham Site (NOBI) and other County Ambient Air Quality Monitoring Stations.

Another site selection factor was the potential of the North Birmingham site data for source attribution analyses. Our initial assessment was optimistic in this regard due to the location of the site relative to several important classes of local and midrange emission sources. The maps in Figures 2 and 3 show some of these sources. As shown in the metropolitan area map in Figure 2, there are three major coal-fired utility stations in the vicinity of Birmingham, located at distances and bearings, respectively, of 24, 39, and $48 \mathrm{~km}$, and 292, 263, and 136 degrees, relative to North. Also shown are some sources to the southwest of the site associated with the iron and steel industry, including a coke plant, steel plant, and steel pipe mill. The locations of the major highways and metropolitan center indicate that mobile source emissions over a large range of directions will impact the site. Figure 3 provides a more detailed view of the area within 5-8 km of the monitoring site, showing several local industrial emission sources. As the figure shows, the North Birmingham site is centrally located relative to these sources, promising good separation of the contributions of at least three clusters of sources with changes in wind 
direction. Roughly in a line to the Northeast (bearing about $45^{\circ}$ ) are two coking plants, a mineral fiber plant, and a cast iron pipe foundry. To the Southeast (bearing from 115-135²), another mineral fiber plant and two iron and steel sources are located within $3 \mathrm{~km}$ of the site, roughly in line with one of the coal-fired power plants. Toward the Southwest (bearing from 230-245) are the iron and steel industry sources shown in Figure 2, as well as a cast iron pipe mill 2-km distant. No other significant local sources lie in the West to Northwest quadrant containing the remaining two coal-fired power plants. 


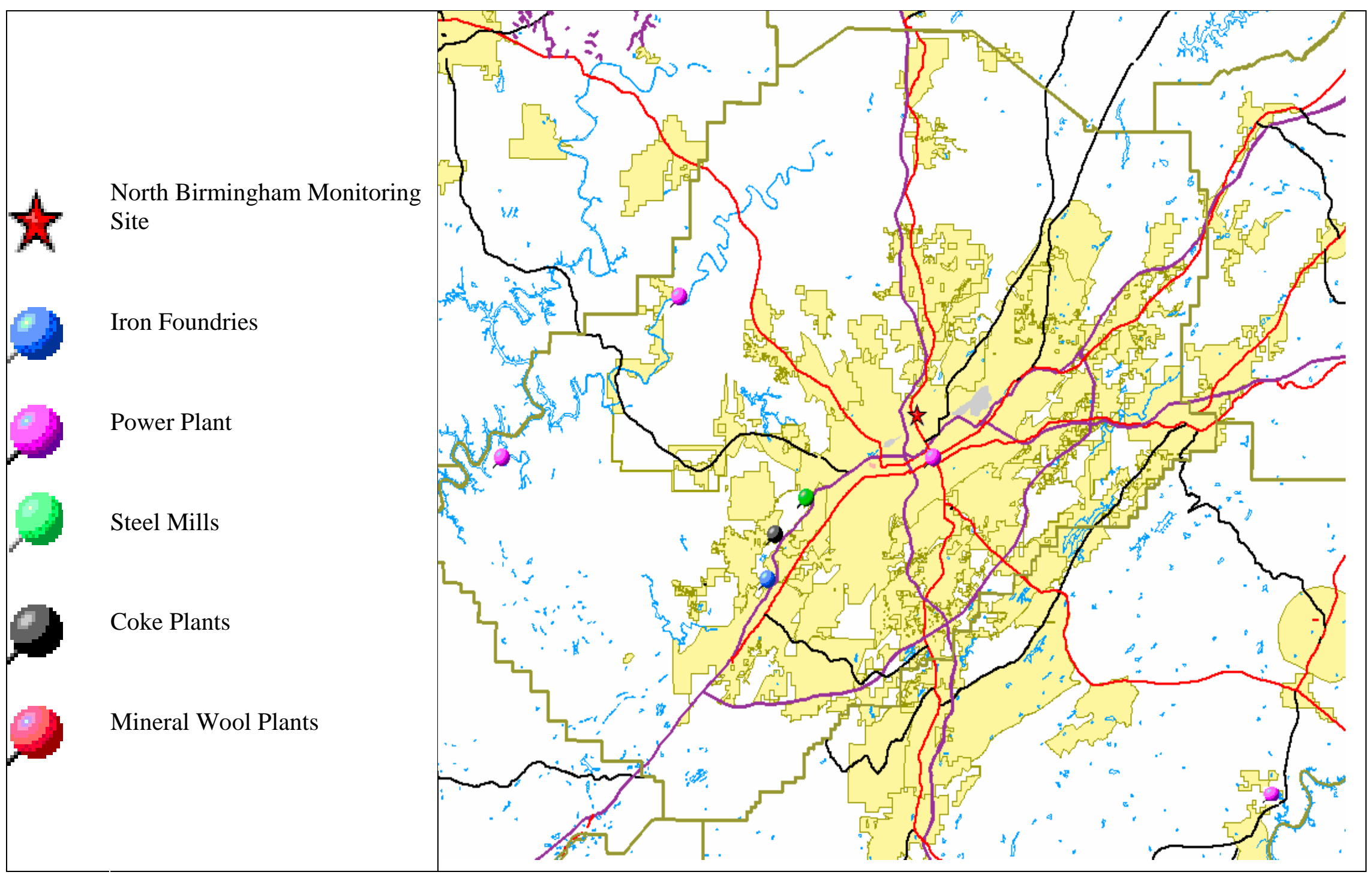

Figure 2. Map of Birmingham Metropolitan Area showing N. Birmingham site and sources outside of the city limits. 


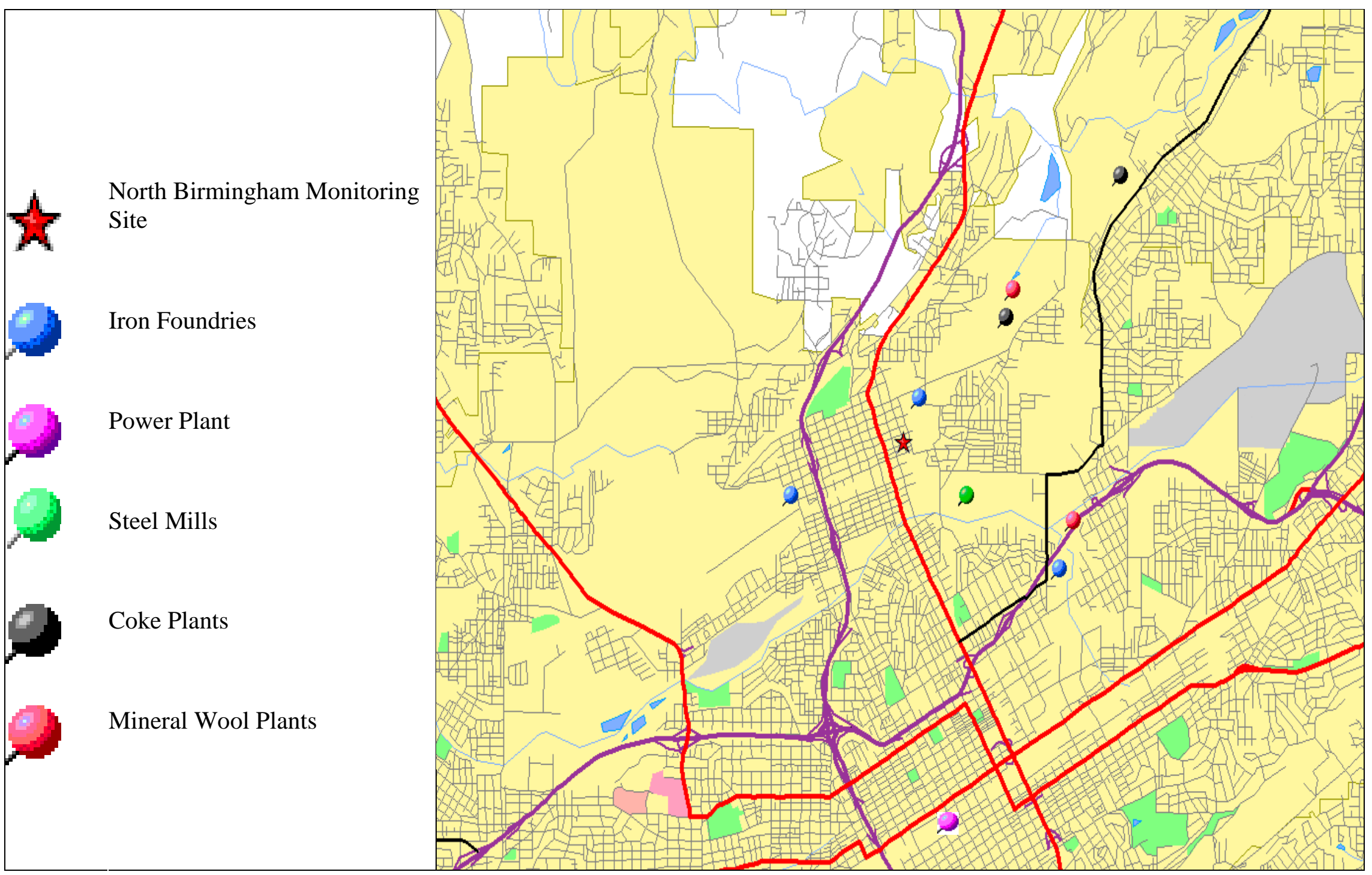

Figure 3. Expanded map of Downtown Birmingham showing monitoring site and local industrial sources. 


\section{Experimental}

\section{Site and deployment arrangements}

Figure 4 is a photograph of the North Birmingham monitoring station. Several filter-based particulate monitors are deployed on the (3 m elevation) platform; those visible on the near side of the platform are operated by the JCHD, as are the TEOM and ozone monitors in the shelter beneath the platform in the front of the figure. The shelter installed for this project can be partially seen on the right side of the picture behind the two storage buildings on that side. A side view of the eastern end of the station before this shelter was installed is shown in Figure 5 . The shelter and towers shown in the right side of this figure were installed by ARA for the SEARCH program, and the larger section of the platform over this shelter holds samplers used for SEARCH. Attached to the left side of this shelter is an isolated "pump shed" used to house vacuum pumps for both the SEARCH monitoring instruments and those installed for the current project. At the front of the figure is shown selected location of the shelter which was installed for the current project, as discussed further below.

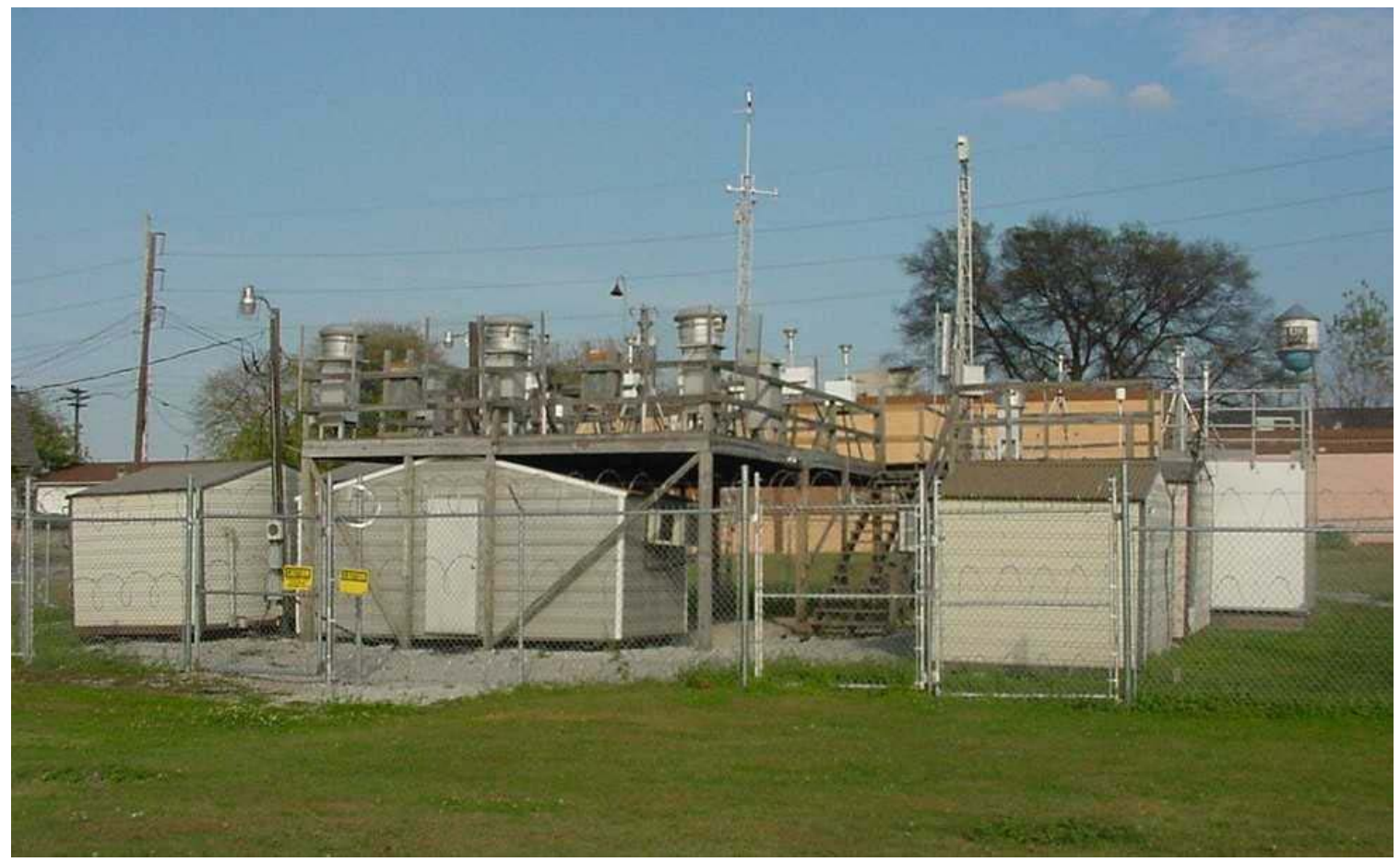

Figure 4. Photograph of the North Birmingham Monitoring site managed and maintained by the Jefferson County Health Department.

In the initial planning for this project, it was anticipated that the shelter to be brought to the site for the EPRI SEARCH program would have sufficient extra space for instruments from this project, which was offered for our use. Accordingly, we planned to deploy our package in that shelter, realizing considerable savings in time and infrastructure cost. Afterwards, this plan became unrealistic for two reasons. First, the shelter available to the SEARCH project was smaller than initially planned, reducing the available extra space. Second, increases in that project budget allowed addition of new measurements not originally planned. As a result, 
additional shelter space not anticipated in initial planning was needed to accommodate all anticipated instruments for the project.

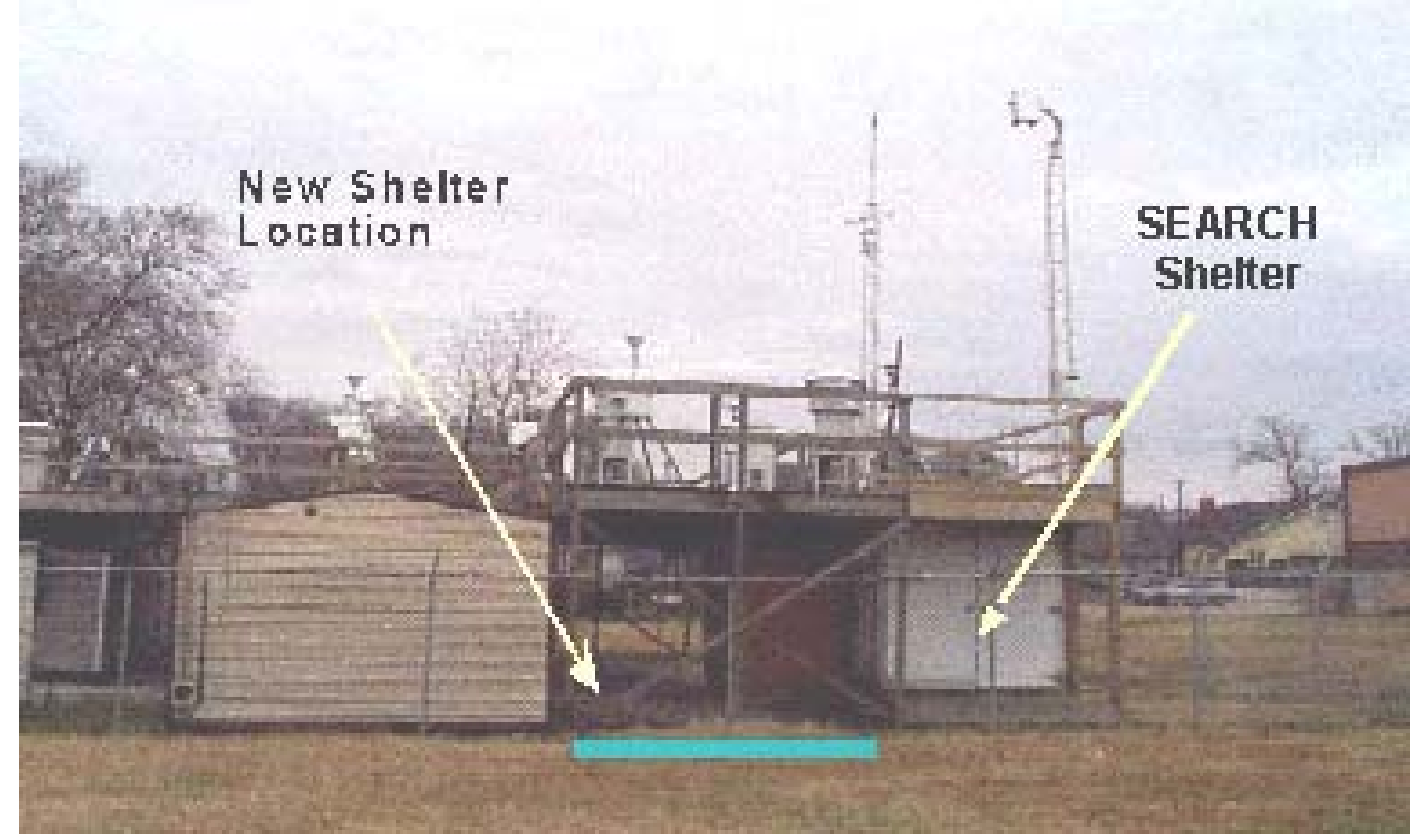

Figure 5. Rear view of the North Birmingham Monitoring site before installation of the sampling shelter for this project, showing the SEARCH shelter and towers.

In order to minimize data loss, the following plan was discussed at the kickoff meeting and agreed to by all parties. First, initial instruments in the SRI project package were installed in the SEARCH shelter while an additional shelter was acquired and installed to accommodate the full instrument package for the project. These initial instruments included the $\mathrm{PM}_{2.5}$ TEOM, the particle sizing monitors (TSI Models 3320 APS and 3934 SMPS), all installed by February 13, 2001, and the nephelometer (Radiance Research Model 903), installed April 13. In the interim, plans for the new shelter were developed and reviewed, and infrastructure changes (power, phone lines, changes to platform and fencing) were coordinated with Health Department and ARA staff. The new shelter was installed in early June, and the TEOM and Nephelometer redeployed on June 18 . The R\&P 8400S Sulfate monitor finally was delivered in early June and was installed on June 19, and the particle sizing package on July 2.

\section{Instrument Configuration and Operating Procedures}

The final measurement suite consists of measurements made by five instruments in the Southern Research sampling shelter at the site: a Tapered Element Oscillating Microbalance (TEOM) particulate mass monitor, an integrating nephelometer, two particle sizing instruments, and an R\&P 8400S particulate sulfate monitor. These instruments were mounted in three standard 19 inch racks located along one wall of the shelter as indicated in the floor plan in Figure 6 below. This wall is immediately adjacent to the existing sampling platform containing other particulate samplers operated by Jefferson County or ARA. On the opposite wall of the shelter is a desk, used for the onsite computer and for supplies. 


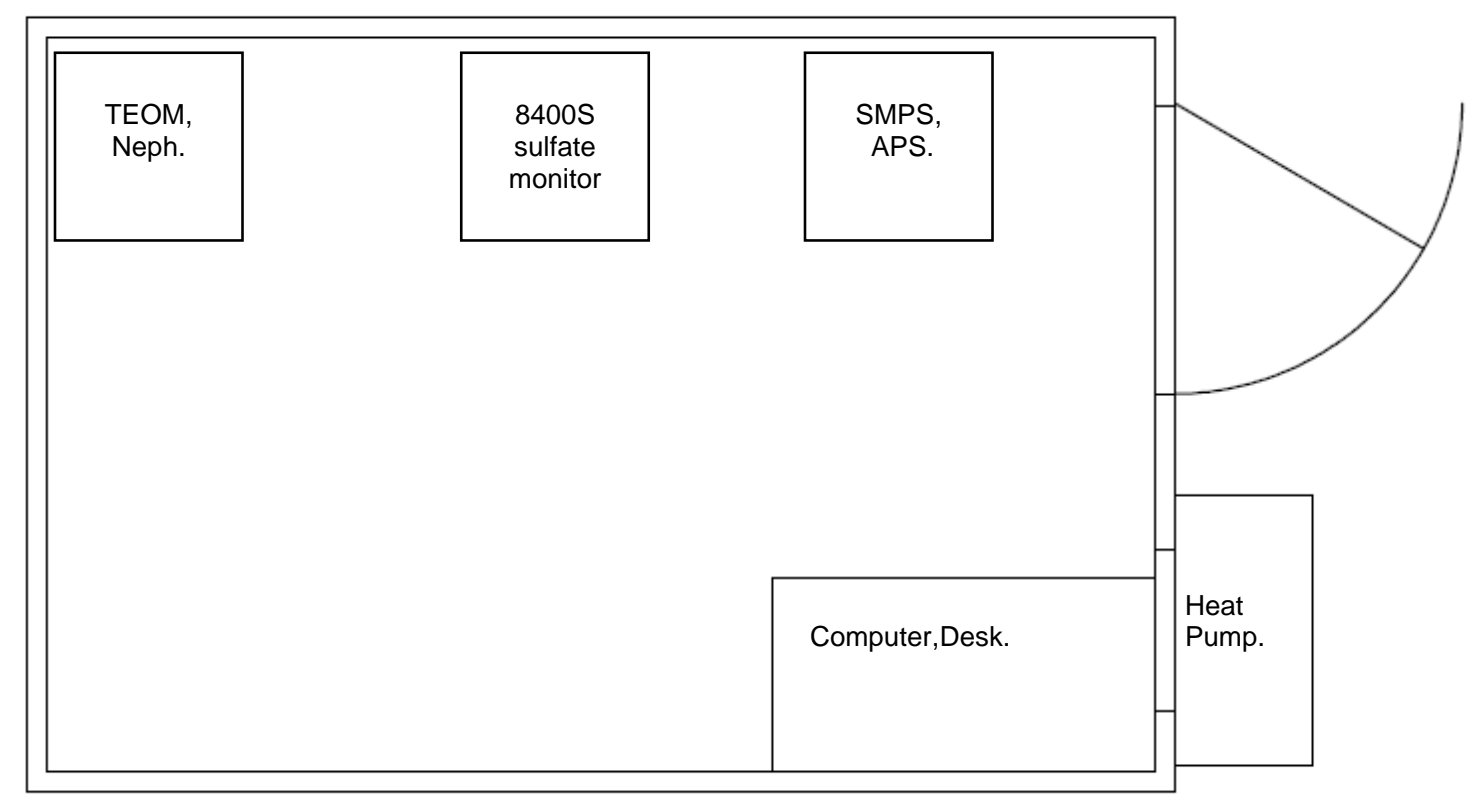

Figure 6. Floor plan of sampling shelter showing location of sampler instrument racks.

All instruments output data in digital form and were controlled by or downloaded automatically via serial communication lines to the onsite computer which collected and stored all raw data in separate files. This computer was polled remotely via modem at least 3 times per week, and the data were assembled at the institute home office and inspected for indications of instrument malfunction. The site was physically visited at least twice per week for routine instrument maintenance and as necessary for intervention or repair. Each instrument has a maintenance schedule that includes replenishment of consumable materials or reagents, cleaning of inlets or sample lines, replacement of filters, etc. at stated intervals or after inspection. Scheduled QA items include checks or adjustment of flowrates, calibration of flow meters, zero/span type calibrations as indicated, and QA review of the collected data.

Figure 7 is a rooftop view of the shelter from the deck of the elevated wooden sampling platform at the site. The perspective here is from the top of Figure 6 facing the bottom of that figure. Along the near side of the shelter roof (white, with metal safety rails) can be seen penetrations leading to the sampler inlets mounted above the three instrument racks depicted in Figure 6. The inlet on the right, which leads to the TEOM/ nephelometer rack, is a standard R\&P PM 10 inlet/ $\mathrm{PM}_{2.5}$ Sharp Cut Cyclone (SCC) unit as typically configured for ambient TEOM PM 2.5 monitoring applications. A similar $\mathrm{PM}_{10}$ inlet (without the SCC) in the left of the figure leads to the particle size monitors below. The stock inlet for the $8400 \mathrm{~S}$ sulfate monitor, partially obscured in the photo, is between the tripod mounts for the other two inlets. 


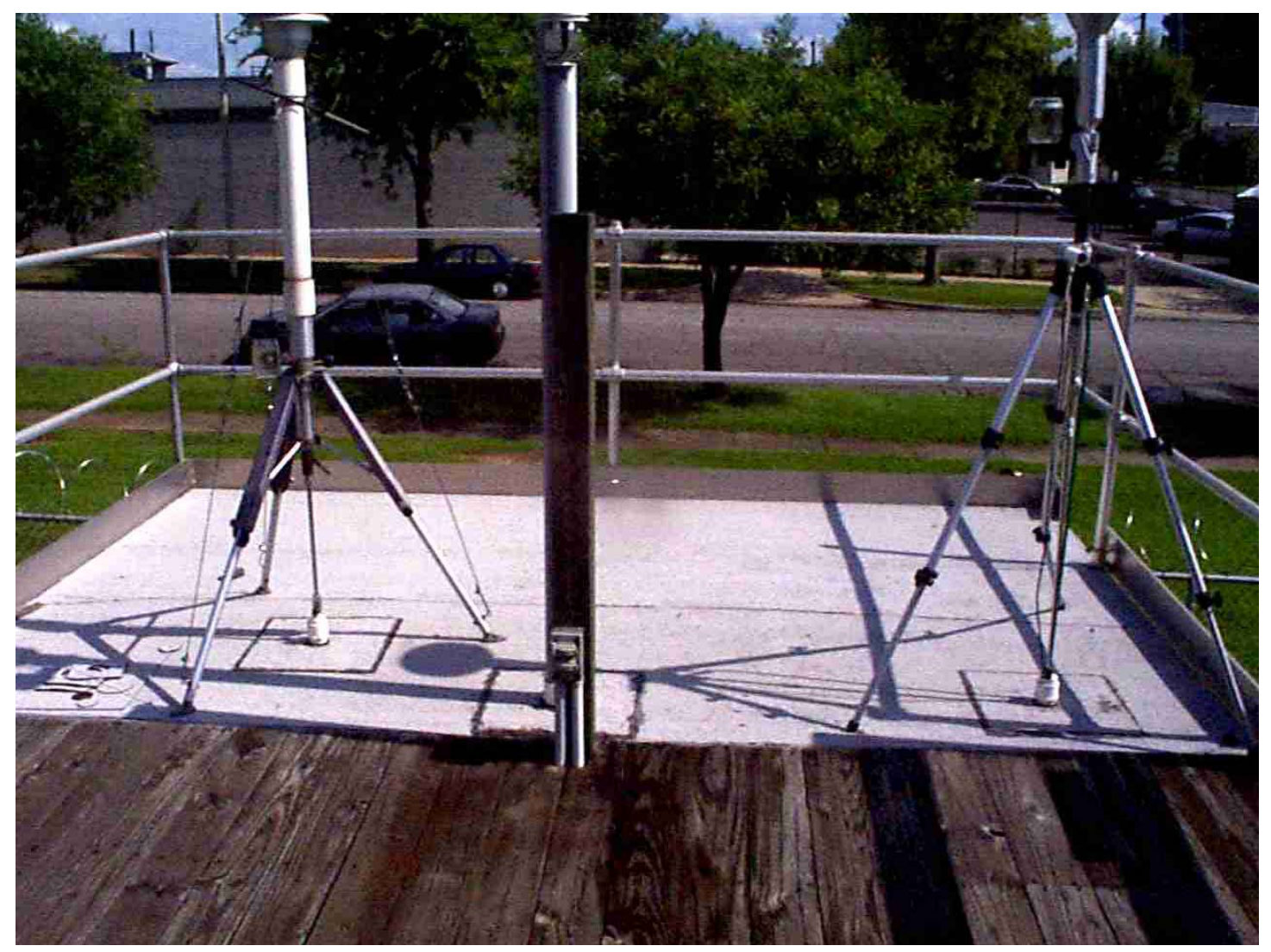

Figure 7. Sampling shelter rooftop view showing sampler inlets .

Figures 8 and 9 below are indoor views of the three instrument racks containing the monitoring instruments deployed inside the shelter. The rack containing the R\&P 8400S sulfate monitor appears in the left of Figure 8 and the right of Figure 9, along with the TSI 3321/3496 particle sizing instrument package (in the right of Figure 8) and the TEOM/Nephelometer rack in the left of Figure 9. The sides and front of this rack were normally covered by insulation board to minimize transients in the TEOM signal that tended to occur when the heat pump cycled on in either heating or cooling mode. For the same reason the TEOM inlet lines are also wrapped in fiberglass insulation, some of which can be seen in the top of Figure 9. Vacuum lines to all instruments are routed along the common wall to a conduit near the shelter door, and passed to the external pump room behind the ARA shelter. Specific configuration and operation details for individual instruments are described below, with emphasis on the features and procedures that are not routine for the devices. 


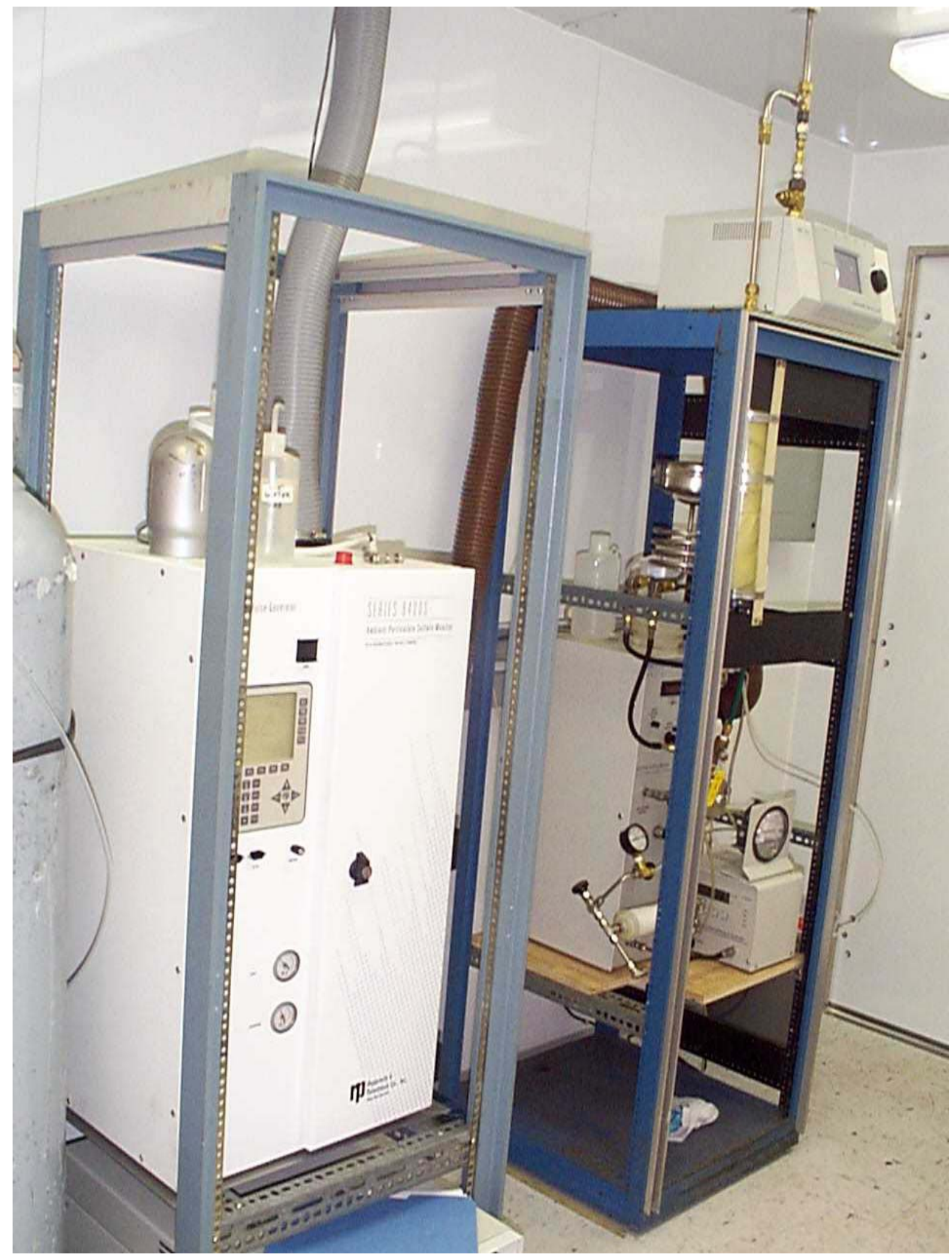

Figure 8. Sampler racks containing R\&P 8400S sulfate monitor (left) and TSI 3321/3496 particle sizing instrument package (right) as deployed inside shelter. 


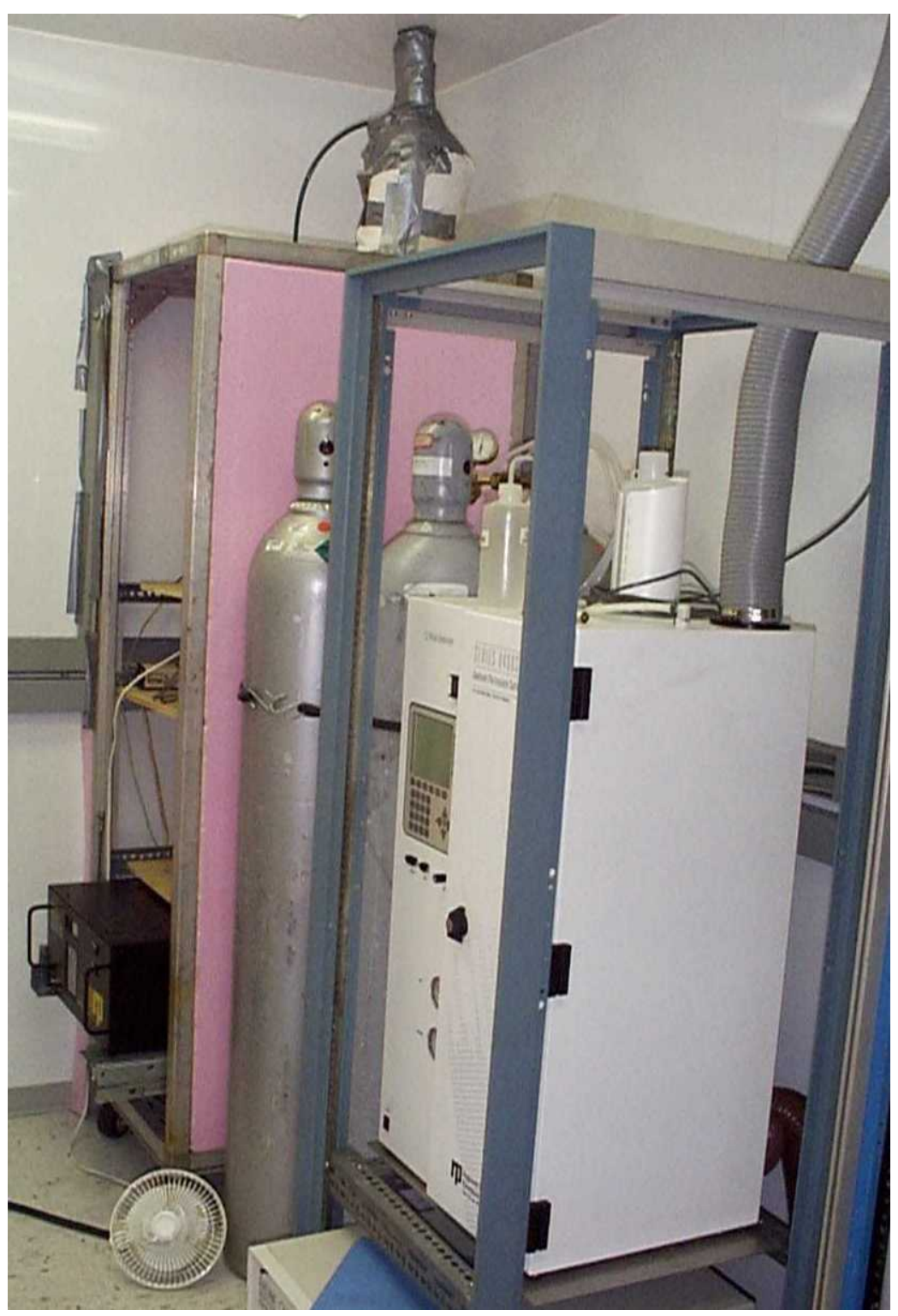

Figure 9. Sampler rack containing TEOM and Nephelometer in left corner beside rack and gas cylinders for $R \& P$ 8400S sulfate monitor.

$\underline{\mathrm{PM}}_{2.5}$ TEOM: The R\&P 1400ab TEOM used in this study is a standard unit equipped with an $\mathrm{R} \& \mathrm{P}$ Sample Equilibration System (SES) dehumidifier and operated at $30{ }^{\circ} \mathrm{C}$ analyzer temperature. The flow configuration is shown in Figure 10, which is adapted from a diagram in the R\&P SES manual. The flow path was modified for our operation in that the 13.7 lpm bypass flow from the inlet is routed through the nephelometer before returning to the TEOM flow control unit. Since the bypass flow is used only to maintain the proper inlet flow rate for the size cuts, this change would not affect TEOM operation unless a leak or flow obstruction occurs in the line. As is shown in the figure, the nephelometer flow can be withdrawn either before or after the SES drier. The SES module has temperature and relative humidity sensors for both sample and bypass (main and auxiliary) flow lines. Main flow temp and RH channel outputs were logged as such, but the bypass line sensors are redundant to the sensors on the Nephelometer, so 
these sensors were removed from the sample line and instead allowed to passively sample the temperature and RH at a point within the rack for the TEOM and Nephelometer units. Since the TEOM has a known susceptibility to temperature fluctuations, these two channels were used to monitor shelter HVAC operation for stability and to identify conditions that could affect instrument performance.

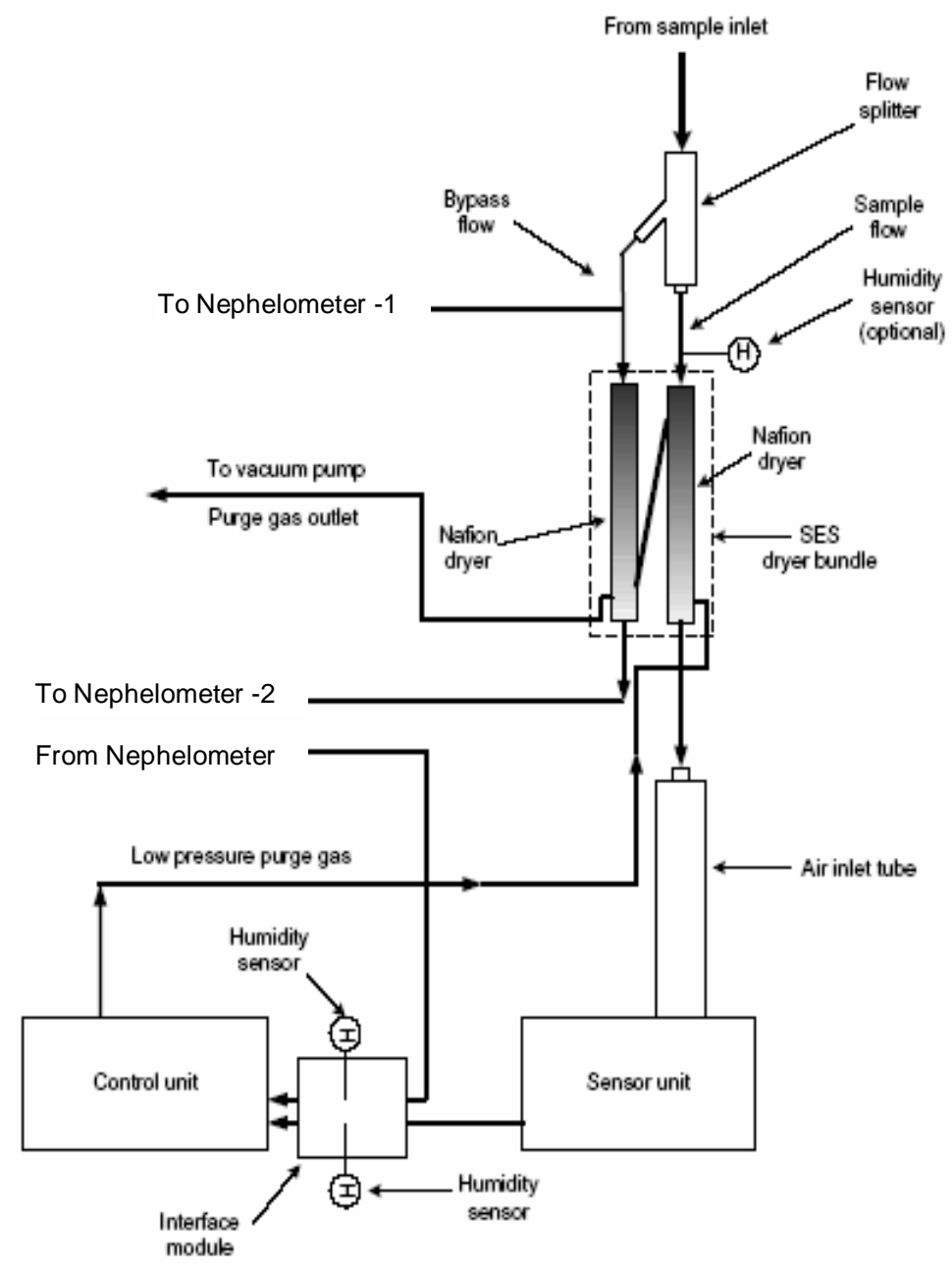

Figure 10. Flow diagram of TEOM 1400ab unit with SES as used in project.

The TEOM unit was operated since installation in the "standard" operating mode defined for its use as an EPA Equivalent Test Method for $\mathrm{PM}_{10}$ - that is, sample flowrate is controlled to be 3 liters/min at actual temperature and pressure, but the reported mass concentrations are expressed in terms of standard temperature and pressure conditions (STP $-25^{\circ} \mathrm{C}$ at $1 \mathrm{~atm}$ pressure). Further, the internal linear correction equation was applied in the instrument firmware. (In terms of instrument setup parameters, this corresponds to values of $99^{\circ} \mathrm{C}, 9 \mathrm{~atm}, 25^{\circ} \mathrm{C}, 1 \mathrm{~atm}, 3$, and 1.03, respectively, for the parameters T-A, P-A, T-S, P-S, Constant A, and Constant B). Other setup choices are possible for TEOM operation, especially since $\mathrm{PM}_{2.5}$ is defined for regulatory purposes in terms of local conditions and the Federal Reference Method (FRM) $\mathrm{PM}_{2.5}$ sampler is operated at constant actual volumetric flow rate. In fact, the $\mathrm{PM}_{2.5}$ TEOM operated by Jefferson County at the site beginning in July, 2001, reports continuous $\mathrm{PM}_{2.5}$ at local temperature and pressure conditions (in terms of instrument setup parameters, T-S and P-S, like T-A and P-A, 
are set at $99^{\circ} \mathrm{C}$ and $9 \mathrm{~atm}$, respectively). The county TEOM further differs from our device in that it is operated in the more common $50^{\circ} \mathrm{C}$ internal temperature mode, filter changes are initiated by high pressure drop conditions rather than scheduled weekly, and the wait time parameter is reduced from the $1800 \mathrm{~s}$ default value used on our instrument. The last two differences allow the JCHD to reduce down time, but may lead to measurement differences immediately after filter changes and perhaps later in the operating life of individual filters. Like our instrument, the county TEOM is operated using the internal correction equation with the default values for the constants described above.

TEOM data are stored digitally in the instrument and downloaded with 1 minute sample resolution. This time frequency is consistent with our goal of near-continuous response, although we recognize the internal averaging time of the instrument is closer to 5 minutes.

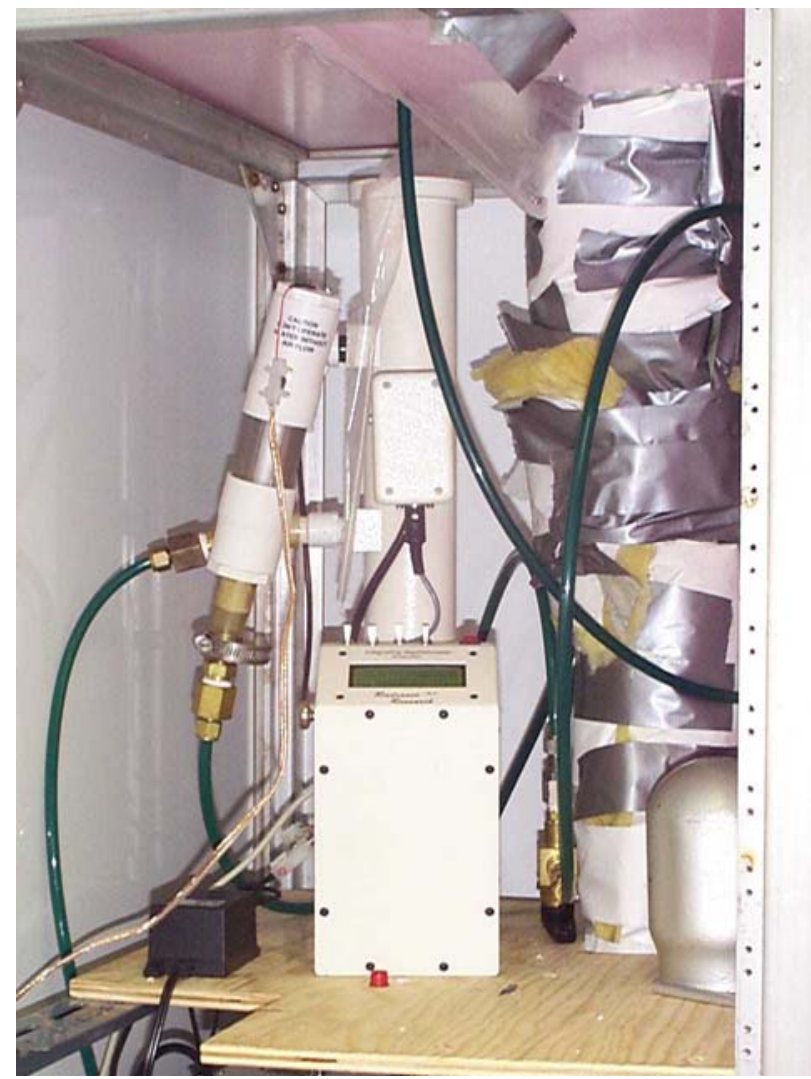

Figure 11. Radiance 904 Nephelometer and "smart heater" relative humidity controller.

Nephelometer: as described above, the Radiance 904 nephelometer operates downstream of the TEOM PM 2.5 SCC in the $13.6 \mathrm{lpm}$ bypass line of the TEOM system. Figure 6 shows the nephelometer and Radiance "smart" humidity controller as connected in the instrument rack. The gas inlet could be switched (using the three-way valve partially visible at the right of the nephelometer body) to sample the low RH air downstream of the SES drier, or to sample upstream of the drier at ambient absolute humidity, with the corresponding relative humidity determined by the roughly $23^{\circ} \mathrm{C}$ shelter temperature (or up to a limiting relative humidity controlled by the heating element in the Radiance "smart" humidity controller). However, during the period of this study, data were collected only in the dry mode downstream of the SES. 
The nephelometer was operated in its logger mode with all default settings for this mode other than the sample logging interval, which was reduced to 1 minute sample periods, consistent with the operating cycles of the TEOM and APS. In this mode data are stored digitally in the instrument and downloaded to the onsite computer on a daily schedule using a serial communications software routine written at SRI. This download is in the form of the nephelometer's "short format" download.

Since the gas flow for the nephelometer was controlled by the TEOM controller, the nephelometer data are invalid during periods of TEOM maintenance such as leak checks, flow audits, filter changes and $\mathrm{K} 0$ calibrations. These periods were noted in the maintenance log, and the corresponding records manually flagged in the nephelometer database.

Particle Size Distribution Measurements: For the two particle sizing instruments we used a consistent protocol after installation in the sampling shelter at the beginning of July 2001, summarized as follows. Submicron size measurements are performed using the TSI, Inc. Model 3496 Scanning Mobility Particle Sizer (SMPS), which measures particle size by electrical mobility. The SMPS operated with continuously repeating 5 minute scanning cycles. The flow range $(0.2 / 2 \mathrm{lpm})$ and scan time were selected for a $20 \mathrm{~nm}$ - $1000 \mathrm{~nm}$ physical diameter size range, and full spectra at particle size resolution of 32 channels/ decade are retained over this range. Larger sizes ( $>0.54 \mu \mathrm{m}$ aerodynamic) are sampled using the TSI Aerodynamic Particle Sizer (APS), which counts individual particles segregated by aerodynamic equivalent diameter using a time-of-flight method. (As described below, the original instrument was a TSI Model 3320; this was upgraded to a Model 3321 in early 2002 when that upgrade became available) Spectra are accumulated continuously at 1 min cycle resolution. The APS and the Nephelometer are both capable of tracking events at this time resolution. APS spectra are retained at particle size resolution of 32 channels/ decade over the range of 0.54 - $20 \mu \mathrm{m}$ aerodynamic diameter, though particles with diameters over $10 \mu \mathrm{m}$ are subject to sampling line losses, poor counting statistics and artifact "ghost" particle counts, and therefore not generally analyzed. The same effects introduce biases in the coarse $(2.5-10 \mu \mathrm{m})$ size range and limit the use of the APS for quantitative measurements in this range. Each of these instruments is controlled by the TSI AIM software on the onsite computer, which collects and stores data directly from both instruments.

The instruments share a common sample inlet system designed for this study. The inlet system described below was installed in the dedicated shelter at the beginning of July, 2001; during the preceding three month period in the SEARCH shelter, a functionally similar inlet system was used with an omnidirectional inlet head constructed at Southern Research facilities. Approximately $3.2 \mathrm{lpm}$ of ambient air is drawn through an R\&P $\mathrm{PM}_{10}$ sampling inlet, which is predicted to have a higher native cutpoint and sample transmission when operating at $20 \%$ of its nominal design flowrate. As can be seen in the top of Figure 8, the sample path descends into a flow splitting assembly directly coupled to the APS sample inlet. The $1 \mathrm{lpm}$ APS sample flow is sampled from the center of the flow line to minimize particle losses, while the remaining air flows in an annulus to the tube in the figure which exits horizontally and passes downward through the top of the rack housing to the diffusion drier, with glass wool thermal insulation visible, that is attached to the near front rack frame member. This drier is actually a TEOM SES unit, only the main flow path of which is used here. The $4 \mathrm{lpm}$ sheath air flow for the APS is drawn from air in the shelter through the small elbow fitting just above the APS in the figure.

The remainder of the SMPS flow path can be seen in Figure 12. Sample air exits the diffusion drier to a "cross" fitting partly visible in the figure. Most of the flow passes through the $1 / 4$ inch 
stainless tubing extending from the right leg of the cross to the stainless steel cylindrical filter assembly mounted to the sheath air inlet on the right front of the SMPS body. This is the $2 \mathrm{lpm}$ sheath air flow used by the device the other two legs are alternate paths to the sample inlet as determined by the position of the three-way valve mounted to the top of the SMPS sample inlet impactor stage. The direct path, which passes downward to the right side of the valve, is not used for normal sampling operation in this study. Rather, the $0.2 \mathrm{lpm}$ sample flow passes left through a one liter volume mixing chamber (partly obscured in the figure) before entering the left side of the three-way valve to the SMPS sample inlet impactor. The purpose of the chamber is to provide a physical time averaging "buffer" whose time constant is comparable to the five minute SMPS scan period used here. This configuration and operation mode was designed to obtain the broadest size range, including the largest particle diameters, feasible for the single mobility tube available with the present unit. This choice entails sacrifices of sensitivity, sample time resolution, and the lower boundary of the particle size range.

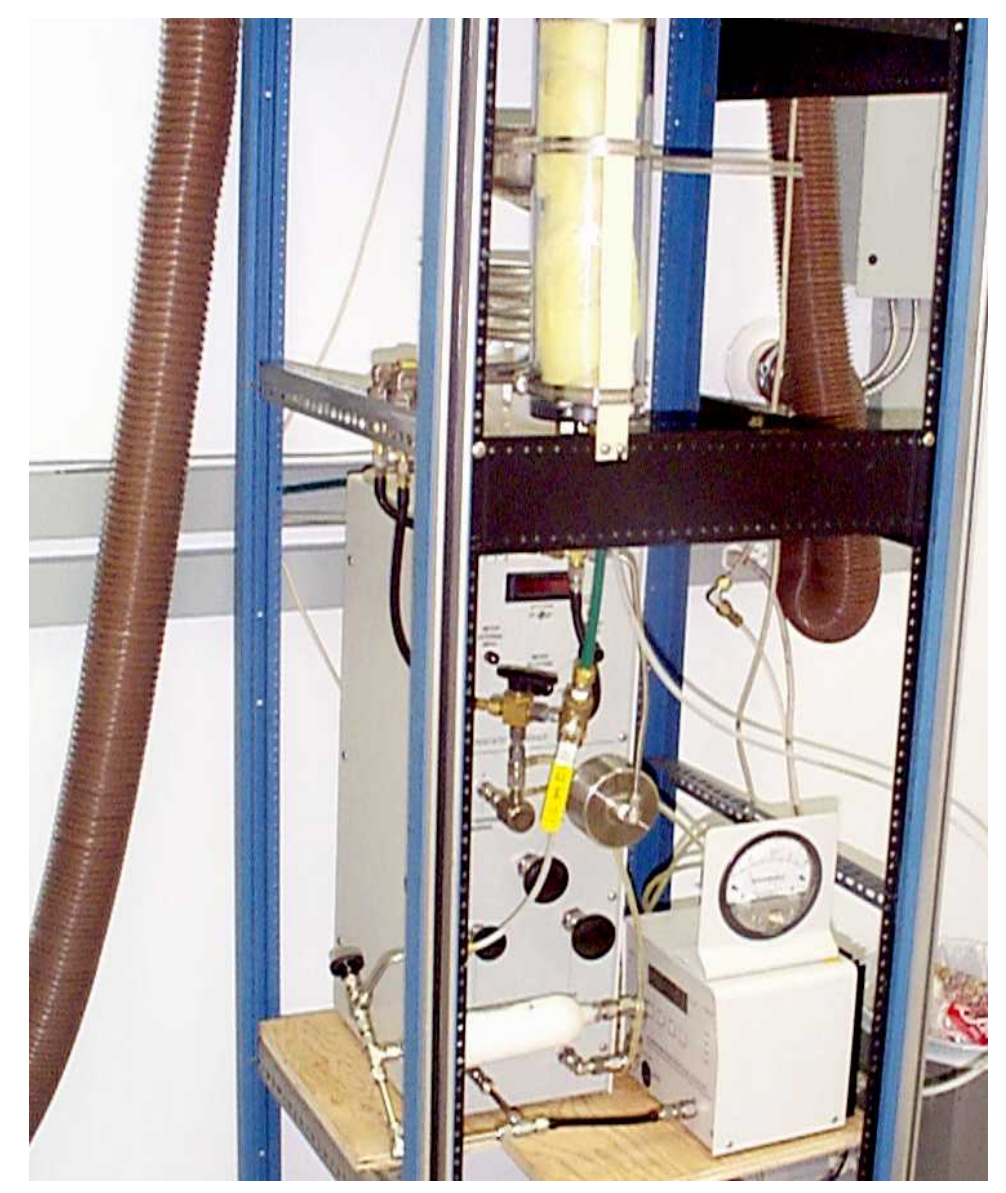

Figure 12. Sample inlet system for SMPS monitor.

The APS was the only instrument that underwent significant modification during the project. After we acquired the original APS Model 3320, TSI introduced the redesigned Model 3321 which was available as an upgrade. This redesign was intended to overcome two reported limitations of the APS 3320; that is, reduced counting efficiency at higher count levels and artifact counts in the largest size bins (especially over 5-10 $\mu \mathrm{m}$ ). Both effects were noted in our data, so we made arrangements with TSI to upgrade our unit APS 3320 to an APS 3321, which 
was done in January 2002. Prior to the upgrade several operational issues were noted. Much of the data during the months of July and August, 2001 had high dead time readings, indicating the possibility of counting efficiency artifacts to which the 3220 was subject. On September 13, 2001 the measured concentrations abruptly dropped and remained at low levels until operational halt on October 30 with a plugged sampling nozzle.

The instrument was removed from the North Birmingham site and returned to SRI for routine cleaning and testing. The inner and outer nozzles of the APS were removed, thoroughly inspected and cleaned. After the cleaning, the instrument's performance was verified by creating an aerosol with 3 different polystyrene latex standards within the instrument's detection limit. All three standards fell within the proper size channels of the APS. The instrument was redeployed to the air monitoring station 11/2/01. During the next three months the gas flow path remained stable; however the sampling efficiency of the device relative to other PM monitors did not recover to the original levels until the upgraded instrument was reinstalled at the site in midFebruary. Some promising changes in instrument performance were immediately noted. The dead time in the upgraded 3321 was reduced by three orders of magnitude (from around 67,000 units to 65) from its predecessor, promising reduced coincidence loss and better response. The Event 4 parameter, indicating "phantom" large particles, was similarly cut by three orders of magnitude. More significantly, the count rate corresponding to submicron particles immediately increased by roughly a factor of five. More specific instrument comparisons will be discussed further in the results section.

As mentioned above, both particle sizing instruments were controlled by the TSI AIM software on the onsite computer, which collects and stores data directly from both instruments. This arrangement, though necessary, led to some data vulnerabilities. The remaining instruments have internal data logging and some power fail recovery capability, and generally have limited data loss on power interruptions and similar incidents. The AIM software control will be interrupted by these events or other computer "hang-ups", and data acquisition will cease until the program is restarted by the operator. Data availability is therefore lower for these instruments.

On several occasions, the instruments and the AIM software were not able to maintain complete time synchronization during data acquisition. For the SMPS, this resulted in truncated scans that were reported as zero concentration in the output and had to be removed and flagged during data screening. For the APS, output data quality was apparently unaffected, but the time base was distorted for certain periods. The operating specifications selected for the APS were sample periods beginning every minute, each involving $55 \mathrm{~s}$ of sample time, allowing $5 \mathrm{sec}$ for processing and recovery by the computer. If the computer could not meet this schedule, the start time would slip and the time between samples became slightly greater than a minute. (The sample collection time was later reduced to $50 \mathrm{~s}$ in an attempt to mitigate this problem). On other occasions, the samples terminated early and the time between samples was slightly less than a minute for extended parts of a sample run (sample runs were generally restarted twice weekly during site visits, but shorter and longer run periods were common). The APS output data quality was apparently not compromised, as the instrument normalizes concentrations for accumulated "live time"; however, the sliding output time can complicate comparison with other instrument data, especially synchronization with other one minute time samples. Our approach to this issue for data processing is described in the next section.

Particle Sulfate Measurements: The R\&P 8400S sulfate analyzer is a commercial version of the flash volatilization ambient sulfate monitor developed by Suzanne Hering of Aerosol Dynamics, 
Inc. and is operated in its typical mode for this project. The monitor operates continuously with a 10 minute cycle time, of which the first 485s is sample acquisition time, and the final 2 minutes are the analysis portion. Daily analyzer audits were scheduled at $3 \mathrm{AM}$ with a standard gas mixture of $\mathrm{SO}_{2}$. These serve as equivalent span measurements and analyzer zero corrections are built into each sample cycle. Periodic aqueous calibrations with an ammonium sulfate standard solution were scheduled at least every two weeks, and conducted weekly when possible.

Like the R\&P TEOM monitor, the 8400S has an internal datalogger from which internal data from the monitor can be downloaded using the vendor's RPComm software. This was done on a twice daily schedule to the onsite computer; the R\&P format text files were transferred to the Southern Research facilities for screening and compilation. 


\section{Results and Discussion}

In the course of this two year project, SRI obtained extensive measurements of ambient fine particulate matter in the Birmingham, AL metropolitan area during the period February, 2001 to September, 2002. As described in the introduction to this report, the experimental portion of this work was guided by the following project objectives:

1) Augment existing measurements of primary and secondary aerosols at an established urban southeastern monitoring site

2) Make a detailed database of near-continuous measurements of the time variation of fine particulate mass, composition, and key properties (including particle size distribution)

3) Apply the measurements to source attribution, time/transport properties of fine PM, and implications for management strategies for $\mathrm{PM}_{2.5}$

4) Validate and compare key measurement methods used in this study for applicability within other $\mathrm{PM}_{2.5}$ research by DOE-FE, EPA, NARSTO, and others.

This project complemented the existing monitoring instruments at the site with a suite of measurements designed to obtain continuous real-time data on $\mathrm{PM}_{2.5}$ mass, particulate sulfate composition, particle size distribution, and light scattering coefficient (Objectives 1 and 2 above). The instrument set and operating procedures were detailed in the previous section; in this section the resulting data will be presented and described. The following subsections will present an overview summary of the data, followed by detailed description of the systematic time behavior of $\mathrm{PM}_{2.5}$ and other specific particulate size fractions. Specific subsections are included for particle size distribution, light scattering, and particle sulfate data. These sections will present both the specific results and the outcome of comparative studies of the instruments and measurement techniques involved (Objective 4). The final subsection will address application of the measurements to the practical questions of fine PM generation and transport, source attribution, and $\mathrm{PM}_{2.5}$ management strategies (Objective 3).

\section{Summary of Particulate Measurement Data}

$\underline{\mathrm{PM}_{2.5}}$ mass concentrations

Figure 13 contains daily average $\mathrm{PM}_{2.5}$ mass concentrations, where available, as measured by the project TEOM monitor over the course of the project time period. For comparison purposes, the plot also contains daily PM $_{2.5}$ concentrations measured by the JCHD onsite FRM sampler and reported on the EPA AIRS database. These plots are generally indistinguishable on the scale of the figure, and the FRM data fill in the days for which valid TEOM daily averages are not available. As will be discussed further below, the daily average concentrations show repeated short term fluctuations from values less than 10 to greater than $40 \mu \mathrm{g} / \mathrm{m}^{3}$, superimposed over a long-term trend of higher concentrations from July - November 2001, lower concentrations through April 2002, then repeated higher concentrations for the remainder of the project period. As will be seen, historical data show this variation to be a recurring seasonal effect. 


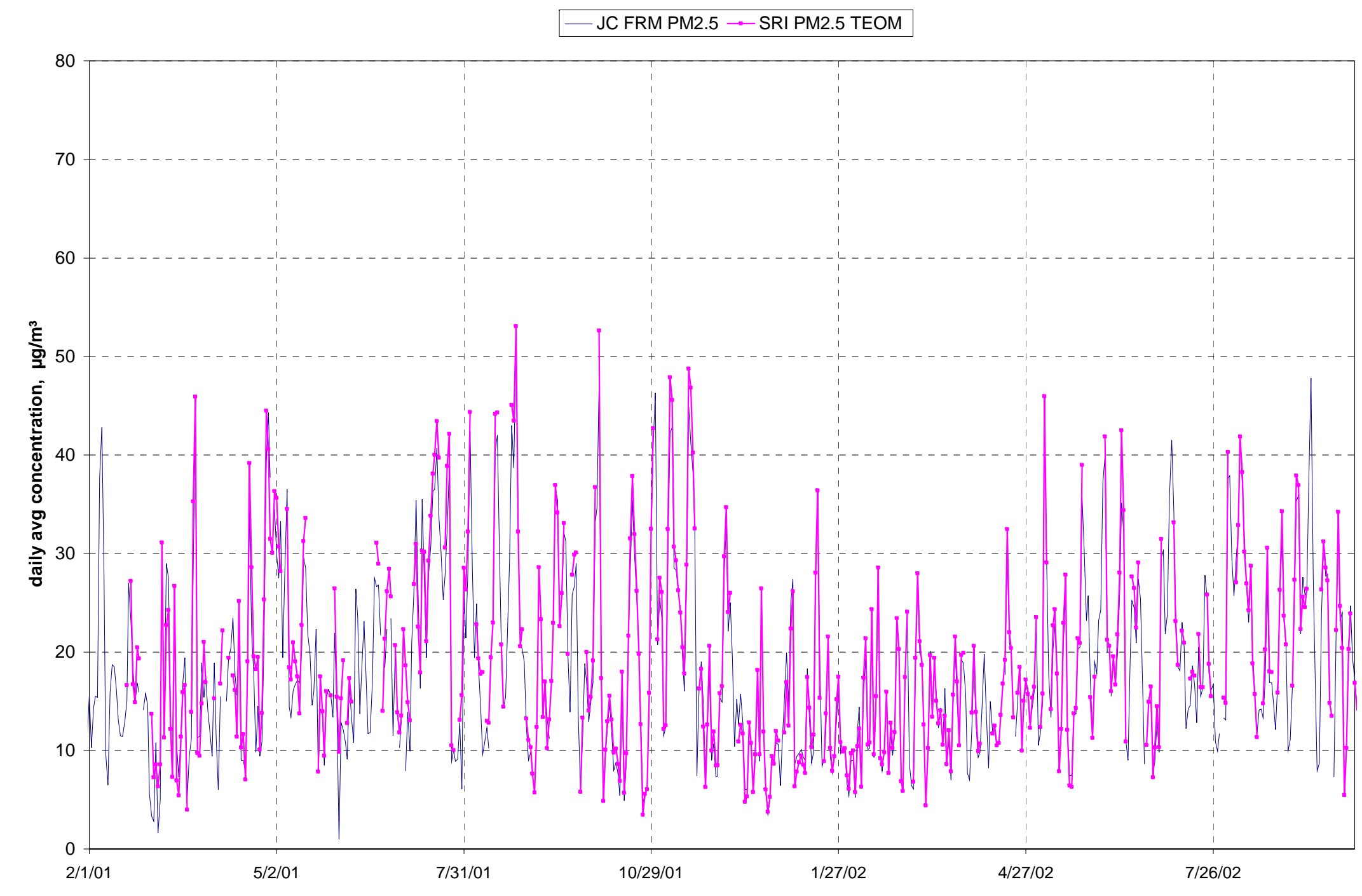

Figure 13. Daily average TEOM PM${ }_{2.5}$ concentration over the project period, with corresponding AIRS FRM PM FI. $_{2.5}$ data. 
The data in this study indicate that the Birmingham Metropolitan area is likely to exceed the $\mathrm{PM}_{2.5}$ Ambient Air Quality Standard. Although the data in Figure 13 did not cover a complete calendar year, the average $\mathrm{PM}_{2.5}$ mass concentration over the year July 1, 2001 - June 30, 2002 was $18.5 \mu \mathrm{g} / \mathrm{m}^{3}$, more than 20 percent over the $15 \mu \mathrm{g} / \mathrm{m}^{3}$ annual standard. Of 469 valid daily averages measured over the project period, $60 \%$ exceeded $15 \mu \mathrm{g} / \mathrm{m}^{3}$, 39\% were over $20 \mu \mathrm{g} / \mathrm{m}^{3}$, and $15 \%$ over $30 \mu \mathrm{g} / \mathrm{m}^{3}$. None of the days in the study, however, exceeded the $65 \mu \mathrm{g} / \mathrm{m}^{3}$ daily average standard for $\mathrm{PM}_{2.5}$.

Related quantities: PM sulfate, Bsc, and $\mathrm{PM}_{10}$

Table 1 summarizes monthly average measurements of particulate mass or related properties from three of the continuous instruments used in this project, as well as related data measured by JCHD at the site. The same results are shown graphically in Figures 14 and 15. The monthly plots illustrate the seasonal variations seen in all the major particulate quantities studied here.

Table 1. Monthly average particulate measurement data during project period (all values in $\mu \mathrm{g} / \mathrm{m}^{3}$ unless otherwise indicated).

\begin{tabular}{|c|c|c|c|c|c|c|c|c|}
\hline Month & $\begin{array}{c}\text { SRI PM }_{2.5} \\
\text { TEOM }\end{array}$ & $8400 \mathrm{~S} \mathrm{SO}_{4}$ & $\begin{array}{c}8400 \mathrm{~S} \mathrm{SO}_{2} \\
(\mathrm{ppb})\end{array}$ & $\begin{array}{c}\text { Bsc, } \\
\times 10^{-6} \mathrm{~m}^{-1} \\
\end{array}$ & $\begin{array}{c}{\text { AIRS } \mathrm{PM}_{2.5}}_{\text {TEOM }} \\
\end{array}$ & $\begin{array}{c}\text { AIRS PM }_{10} \\
\text { TEOM }\end{array}$ & $\begin{array}{c}\text { AIRS } \\
\text { calculated } \\
\text { PMc }\end{array}$ & $\begin{array}{l}\text { AIRS PM }_{2.5} \\
\text { FRM }\end{array}$ \\
\hline Jan-01 & & & & & & 26.6 & & 19.5 \\
\hline Feb-01 & & & & & & 32.9 & & 17.6 \\
\hline Mar-01 & 14.6 & & & & & 27.8 & & 14.6 \\
\hline Apr-01 & 20.6 & & & & & 41.3 & & 18.5 \\
\hline May-01 & 22.3 & & & 34.9 & & 42.2 & & 19.9 \\
\hline Jun-01 & & & & 41.2 & & 33.5 & & 17.0 \\
\hline Jul-01 & 26.5 & 6.7 & 2.4 & 62.2 & & 41.0 & & 23.1 \\
\hline Aug-01 & 26.4 & 6.8 & 3.0 & 58.4 & 25.4 & 45.7 & 20.4 & 24.3 \\
\hline Sep-01 & 19.8 & 5.2 & 3.8 & 47.8 & 20.0 & 38.1 & 18.1 & 19.7 \\
\hline Oct-01 & 19.5 & 3.0 & 4.1 & 33.9 & 19.7 & 45.5 & 26.8 & 18.9 \\
\hline Nov-01 & 22.8 & 3.8 & 4.7 & 36.1 & 21.6 & 53.9 & 31.7 & 22.1 \\
\hline Dec-01 & 12.9 & 2.8 & 3.9 & 16.8 & 12.8 & 26.9 & 14.2 & 13.4 \\
\hline Jan-02 & 13.0 & 3.2 & 5.0 & 15.9 & 13.0 & 25.2 & 12.1 & 14.0 \\
\hline Feb-02 & 12.1 & 2.7 & 4.3 & 13.4 & 12.7 & 24.3 & 12.0 & 12.4 \\
\hline Mar-02 & 14.3 & 3.7 & 2.9 & 14.4 & 14.4 & 25.7 & 9.8 & 14.0 \\
\hline Apr-02 & 15.3 & 4.0 & 3.2 & 15.8 & 16.1 & 33.2 & 16.8 & 14.8 \\
\hline May-02 & 19.0 & 4.6 & 2.9 & 24.3 & 20.0 & 36.2 & 16.3 & 18.9 \\
\hline Jun-02 & 21.5 & 4.7 & 4.0 & 26.2 & 21.4 & 43.6 & 22.2 & 20.3 \\
\hline Jul-02 & 21.0 & 6.4 & 4.3 & 37.3 & 21.7 & 37.1 & 15.4 & 20.4 \\
\hline Aug-02 & 24.3 & & & 38.0 & 23.8 & 51.5 & 25.7 & 23.1 \\
\hline Sep-02 & 23.4 & & & 32.2 & 23.8 & 47.5 & 23.5 & 23.1 \\
\hline
\end{tabular}

Figure 14 summarizes monthly average data from the $\mathrm{PM}_{2.5}$ TEOM and 8400S particulate sulfate monitor used in this project. In addition, the figure contains data from two $\mathrm{PM}_{2.5}$ instruments operated by JCHD the FRM daily mass sampler and the JCHD TEOM used to compute the particulate Pollutant Standard Index (PSI) for the site. As described earlier, this TEOM was 
installed in July, 2001 and is operated at the more common $50 \mathrm{C}$ sample head temperature without the SES diffusional drier used on our $30 \mathrm{C}$ sampler. As can be seen in Figure 14, the three measurements track closely except for April - July 2001, when our TEOM averages were $12-15 \%$ higher than the FRM results. For the remaining months of the study, the three devices were more comparable: our TEOM average was 2.2 percent higher overall than the FRM and less than $0.5 \%$ lower than the JCHD TEOM. These results will be discussed in more detail in the section on instrument comparisons. Figure 14 also contains monthly average data from the 8400S sulfate monitor. The monthly average particulate sulfate at the site rises and falls in concert with the total $\mathrm{PM}_{2.5}$ mass, but not proportionally. The sulfate concentration in the first three months of instrument operation averaged $26 \%$ of the total $\mathrm{PM}_{2.5}$ measured by the TEOM; over the next two months, the ratio falls to $16 \%$, rising to intermediate values in later months. The gaseous $\mathrm{SO}_{2}$, measured as an auxiliary output of the 8400S, shows an inverted seasonal pattern. Average $\mathrm{SO}_{2}$ is lowest in the first summer when $\mathrm{PM}_{2.5}$ and particle sulfate are highest, and peak during the winter months when $\mathrm{PM}_{2.5}$ and particle sulfate are lowest.

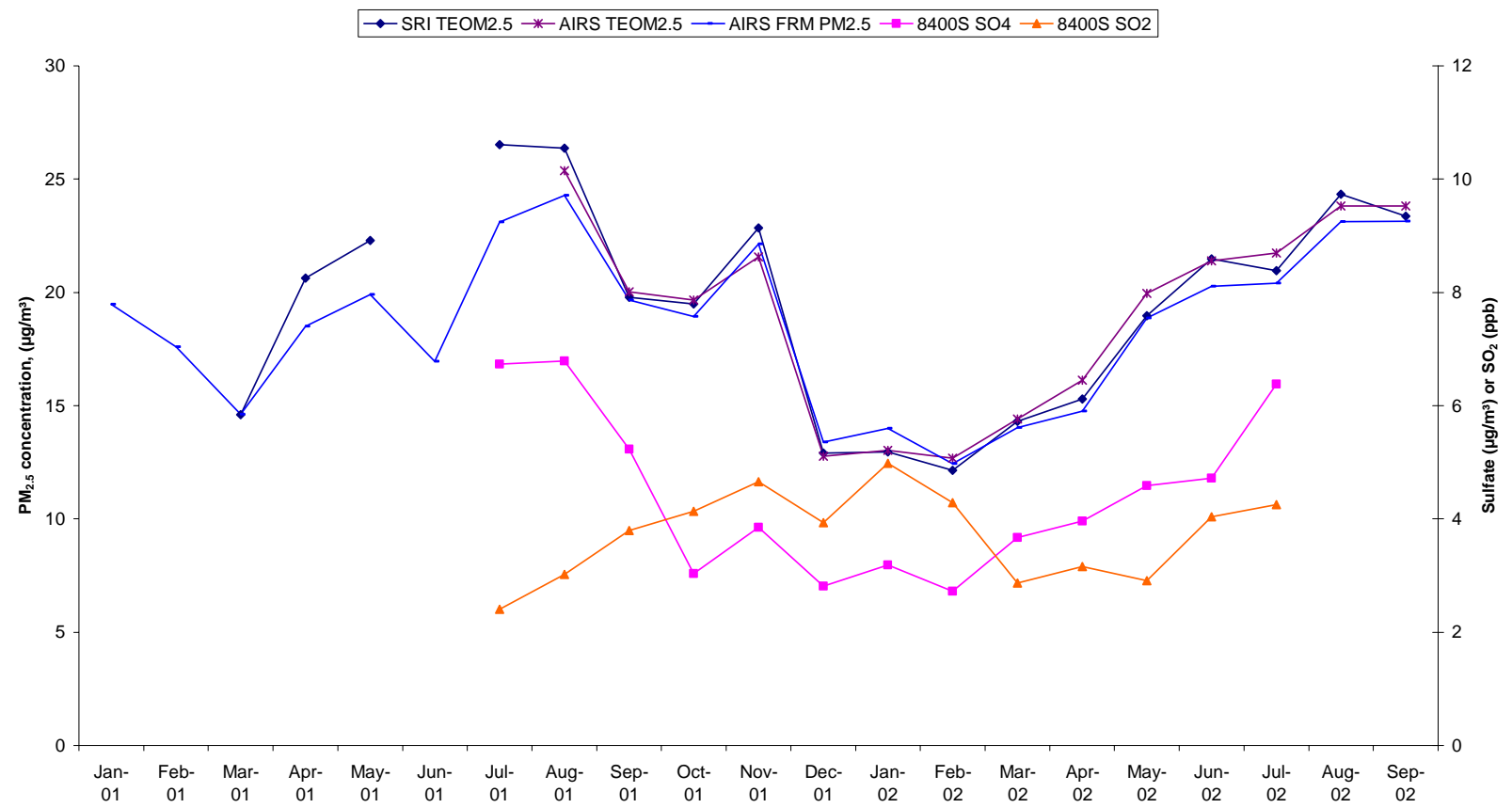

Figure 14. Comparison of monthly average $\mathbf{P M}_{2.5}$ concentration and 8400 S sulfate monitor data over the project period.

In addition to the monthly average data from the two $\mathrm{PM}_{2.5}$ TEOM samplers from Figure 14, Figure 15 contains JCHD data relevant to the coarse particulate fraction. This consists of the $\mathrm{PM}_{10}$ TEOM measurement data from the site, as reported on the AIRS database, and the value of the coarse (PMc, or the 2.5 - $10 \mu \mathrm{m}$ fraction) computed by the difference of the two sampler measurements. For most months in the study period, the computed PMc is approximately equal to or slightly less than the measured $\mathrm{PM}_{2.5}$. The exception to this pattern is the two-month period of October and November 2001. In addition to enhanced $\mathrm{PM}_{10}$ (and consequently PMc), this period has unseasonable values of several other measured quantities. Figure 15 also contains monthly average Bsc values as measured by the Radiance 903 Nephelometer. Like the sulfate data in the previous figure, the average Bsc tracks the average $\mathrm{PM}_{2.5}$ but not linearly. The relative 
nephelometer response per unit $\mathrm{PM}_{2.5}$ appears to be greater in the warmer months. Since ambient humidity and particle bound water are also expected to be higher at these times, one possible explanation is retention of particle bound water in spite of the diffusional drier on the inlet of the nephelometer.

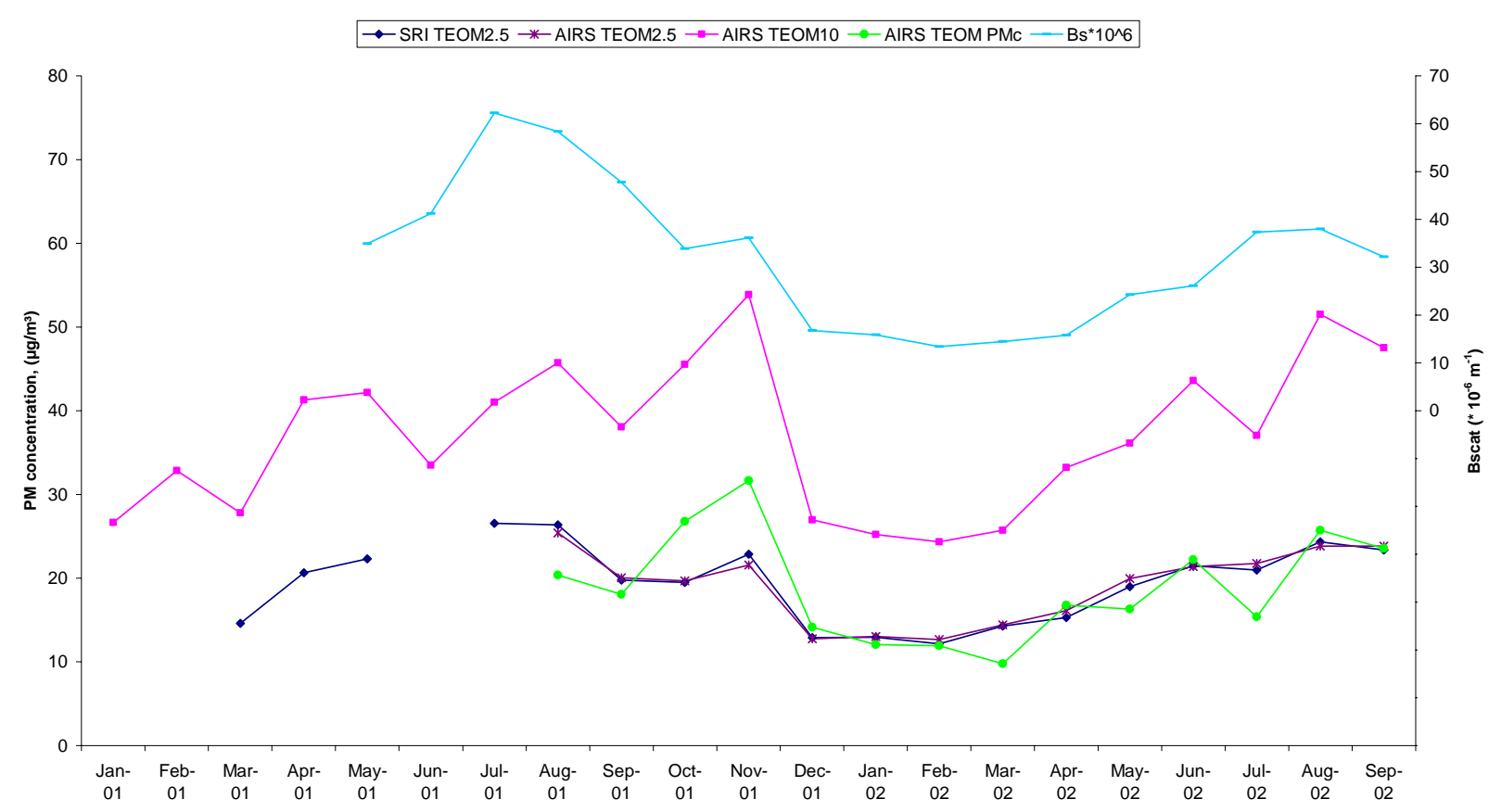

Figure 15. Comparison of monthly average $\mathbf{P M}_{2.5}, \mathbf{P M}_{10}$, and nephelometer data over the project period.

Particle size distribution and size fractions

Figure 16 depicts a composite of the average particle size distribution spectra obtained from the APS and SMPS analyzers during the month of June, 2002. The figure has features typical of particle size over the whole study period, and illustrates several key particle size fractions which will be analyzed in more detail in coming sections. On the left side of the plot is the SMPS particle distribution with differential number weighting, which here shows a single mode with peak at 58nm. The ultrafine particle range, here defined as smaller than $100 \mathrm{~nm}$ physical diameter, invariably contains most particles by number, though typically less than $5 \%$ of the $\mathrm{PM}_{2.5}$ mass (4\% in the period shown). The instruments did not cover particle sizes smaller than $20 \mathrm{~nm}$, which is most sensitive to two notable particle sources. The first are nucleation "events" noted in other studies in the eastern US, in which great numbers of secondary particles formed smaller than $5 \mathrm{~nm}$ which grow into the $30-50 \mathrm{~nm}$ range. Also in this size range are primary particles that are products of combustion from mobile and some stationary sources. While our SMPS is far less sensitive to most of these particles than nano-SMPS instruments, we have monitored several size fraction metrics to enhance detection of these events. These include particle number $<30 \mathrm{~nm}$ (N30 about 6-8\% of our total number) and particle volume fractions under 50 or $100 \mathrm{~nm}\left(\mathrm{PM}_{50}\right.$ or $\mathrm{PM}_{100}$ here; respectively about 0.4 and $4 \%$ of $\mathrm{PM}_{2.5}$, and comparable to data from nano-DMA systems). 
When plotted with mass weighting, the SMPS size distribution in the center of the figure approximates a log-normal distribution which peaks at about $380 \mathrm{~nm}$, a classic presentation of an ambient particulate accumulation mode. The shape of this distribution, with maxima between 300 and 400nm, is reproduced in the other monthly averages during the study, and is qualitatively representative of short- term average size distributions as well. Within this pattern, the mean particle size and the shape of the size distribution in this range vary systematically over the course of a typical day or pollution episode, as will be described in a later section. The overall accumulation mode is the dominant portion of the $\mathrm{PM}_{2.5}$ mass, and typically extends slightly beyond the $1 \mu \mathrm{m}$ size limit attainable with the SMPS in this operating mode.

The average APS size distribution has considerably more structure than the SMPS distribution, with evidence of modes around 3 and $7 \mu \mathrm{m}$ and some contributions from particles larger than 10 $\mu \mathrm{m}$. This general shape is reproduced in other monthly average size distributions after the instrument upgrade in February, 2002. Short-term averages are much more variable, and the size distribution over the PMc clearly is a mixture of source- specific components. Typically, the APS spectra show a minimum between 1 and $2 \mu \mathrm{m}$, consisting of a mixture of the "tails" of the course and the submicrometer accumulation modes. This size region apparently contributes about $15 \%$ of the $\mathrm{PM}_{2.5}$ mass at this site. The APS size spectra have not been corrected for sizedependent sampling losses in the PMc fraction. Accordingly, the integrated concentration of particles between 2.5 and $10 \mu \mathrm{m}$ aerodynamic diameter is only 33\% of the PMc computed from the difference of the $\mathrm{PM}_{10}$ and $\mathrm{PM}_{2.5}$ TEOM hourly averages during the June 2002 period shown in the figure. However, since the two coarse particle measurements track well over short-term periods when the APS is functioning, the APS is still useful to identify size-specific variations in source contributions, especially over short time periods which can be resolved using the oneminute time resolution of the APS. 


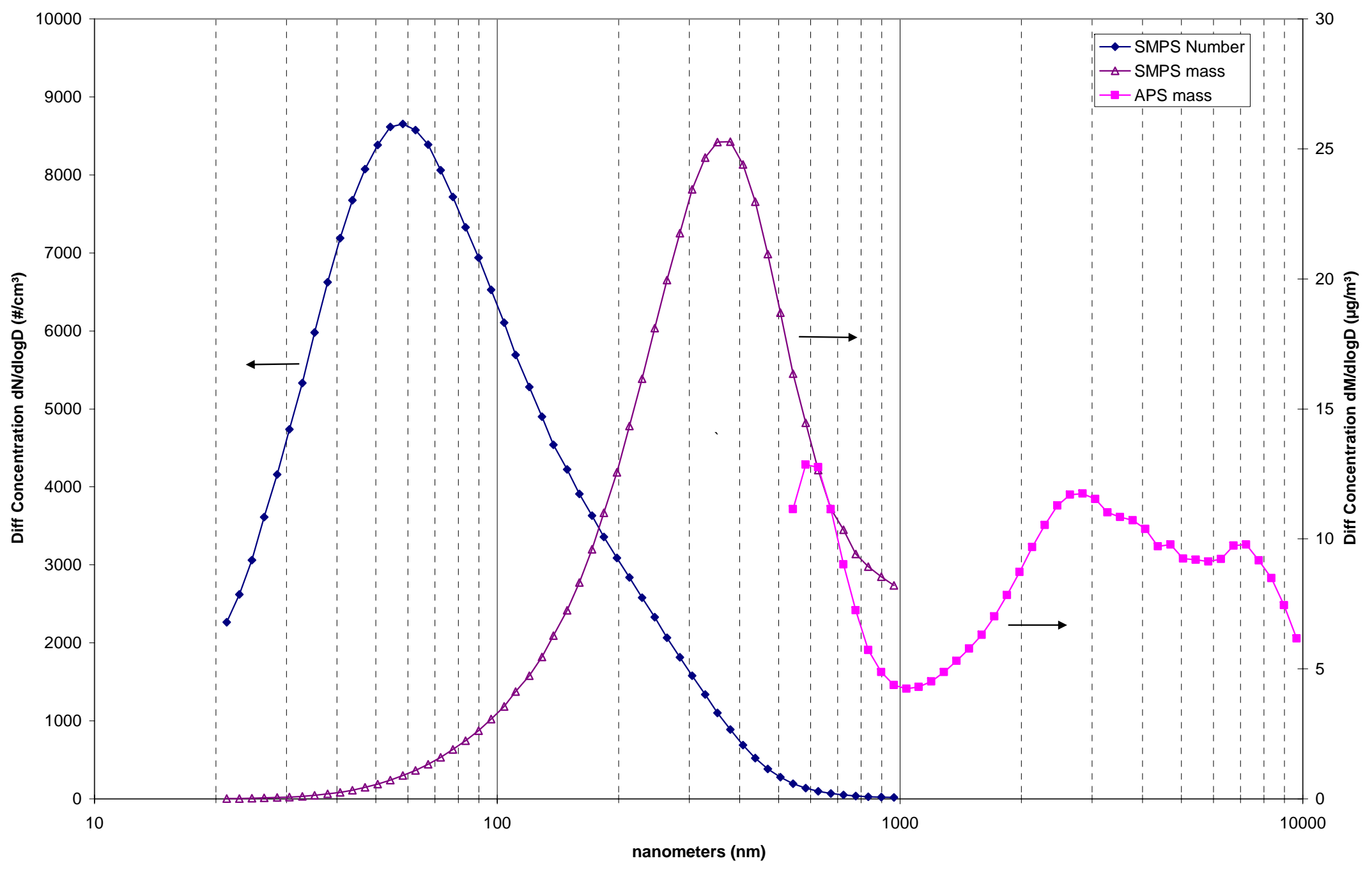

Figure 16. Composite monthly average particle size distribution for June, 2002 as measured by SMPS and APS. 


\section{Time Behavior}

The time variation of particulate matter observed at the study site is complex, and can be described as a mixture of several recognizable patterns on differing short and long time scales. Consideration of the factors and mechanisms of particle generation, transformation, transport, and loss immediately suggests several characteristic time scales that may be important. For example, emissions of primary particles or their gaseous precursors generally vary with characteristic time signatures, often very predictable. At the short-time scales, emissions processes can fluctuate within minutes or less, and frequently vary by hour. Industrial and mobile sources often have a repetitive emission pattern over the course of a day, which can also differ by day of the week. Natural emissions sources (e.g., vegetation, wind-blown dust) also show a time of day and day-to day variation, as well as seasonal time patterns. Anthropogenic sources may also vary seasonally, and may have important longer-term variations as major sources are brought into service, change process outputs, or add emissions controls. There are also characteristic timescales for the atmospheric processes that affect particle formation, transport, transformation and loss. At the shortest times are wind shifts affecting plume transport, and the initiation of rain or nucleation events, which can occur within minutes or less. Other systematic atmospheric processes occur repetitively over the course of a day, and less regularly over the course of several days with the passage of weather fronts or storm systems. Finally, atmospheric processes show a systematic yearly seasonal cycle, but with notable year-to year variation. These longer-term variations are not easily predicted from measurements over shorter periods, but can be of great practical significance for air quality management, as evidenced by the recurring regulatory issues in ozone air quality attainment in major U.S cities.

These considerations suggest that several time scales may be significant for this study:

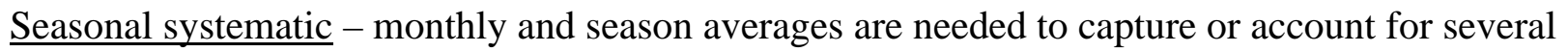
source and atmospheric variations, including:

- Photochemical production or transformations of secondary particulate, especially sulfate and organic carbon fractions

- Production and transport of resuspended crustal particulate matter

- Particle-gas partitioning, particle-bound water and other factors affected by temperature and humidity

Multiday systematic: to capture day of week source variations

Multiday irregular: correlated with meteorological cycles

Daily: to capture or account for regular and irregular source variations, daily meteorological patterns, hour time resolution or better is optimal.

Sub-daily: to analyze or separate irregular source variations, source-specific plume transits, or other short-term events involving nearby sources, sub-hourly time resolution (1-5 minutes) may be required. The continuous measurements in this study were selected to achieve this resolution where technically feasible.

Since the time scales mentioned above, and the required time resolutions, cover a large range, it is a challenge to visually present the time sequence data. An instructive overview can be obtained by inspection of the data over a series of decreasing observation periods, beginning with the plot of daily average $\mathrm{PM}_{2.5}$ over the project period previously shown in Figure 13 . The daily averages in this figure show a recognizable day to day autocorrelation, with a recurring 
pattern of minima and maxima with peak to valley ratios in the range of 2 to 10 . These peaks are spaced by irregular intervals typically on the order of $5-8$ days and generally correlated with the passage of synoptic weather systems, as is frequently seen in time series of other ambient air pollutants. The overall seasonal trend in average $\mathrm{PM}_{2.5}$ concentrations is seen as an irregular envelope over the daily average peaks and valleys, both of which follow the general seasonal trend of the monthly mean concentrations.

Figures 17A and B contain an expanded plots of four month time periods, where now hourly averages of the $\mathrm{PM}_{2.5}$ TEOM are plotted, along with hourly average wind speed measured by the onsite 10 meter met tower operated by ARA. Figure 17A contains a plot of the four month time period from July - October, 2001. This time frame covers the first summer of the study, and shows features also seen in the summer of 2002. The figure shows more clearly the multiday structures as clusters of 2-10 days of elevated $\mathrm{PM}_{2.5}$ concentration separated by multiday intervals of lower concentration. The correlation of $\mathrm{PM}_{2.5}$ trends with meteorological conditions can be seen in the wind speed data in the top portion of the figure. The multiday intervals having elevated $\mathrm{PM}_{2.5}$ concentration generally correspond to intervals with lower mean daily wind speed and very low overnight wind speed. These are characteristic of a recurring summer weather pattern which also lead to episodes of elevated ambient ozone in the Birmingham airshed: episodes of subsidence inversion under the influence of high pressure cells, leading to clear, sunny, stagnant periods lasting several days, during which photochemically produced air pollutants accumulate. It is no great surprise to see that these episodes also include high levels of $\mathrm{PM}_{2.5}$.

The hourly resolution in Figure 17A reveals an added diurnal structure to the daily averages which will be seen more clearly in the next figures. These daily oscillations "ride" on the multiday structures seen in the previous figure, and like the pattern seen at the longer time scales, the multiday trend is followed by the daily maxima and minima as well as the daily mean concentrations. The amplitude of the daily period oscillation in this figure is generally on the same order as that of the multiday variation: during some multiday cycles (like 9/3-15/2001) the daily oscillations are smaller than the daily mean trend; during others (like 10/1-5 or 10/18-23) the daily oscillations dominate. The wind speed data also provide a fairly obvious explanation of these variation patterns. The stagnant overnight conditions during the pollutant episodes allow accumulation of locally produced $\mathrm{PM}_{2.5}$ under the thermal inversion layer that is also characteristic of these conditions. Since the accumulated concentrations are substantially reduced by the increased atmospheric mixing in the daylight hours, the same weather conditions that accompany the highest $\mathrm{PM}_{2.5}$ episodes produce the largest daily oscillations. In contrast, the days when the overnight wind speed remains higher than $0.3 \mathrm{~m} / \mathrm{s}$ correspond to periods of lower diurnal variation of $\mathrm{PM}_{2.5}$, presumably because the atmospheric mixing is greater and not as variable during the daily cycle. 


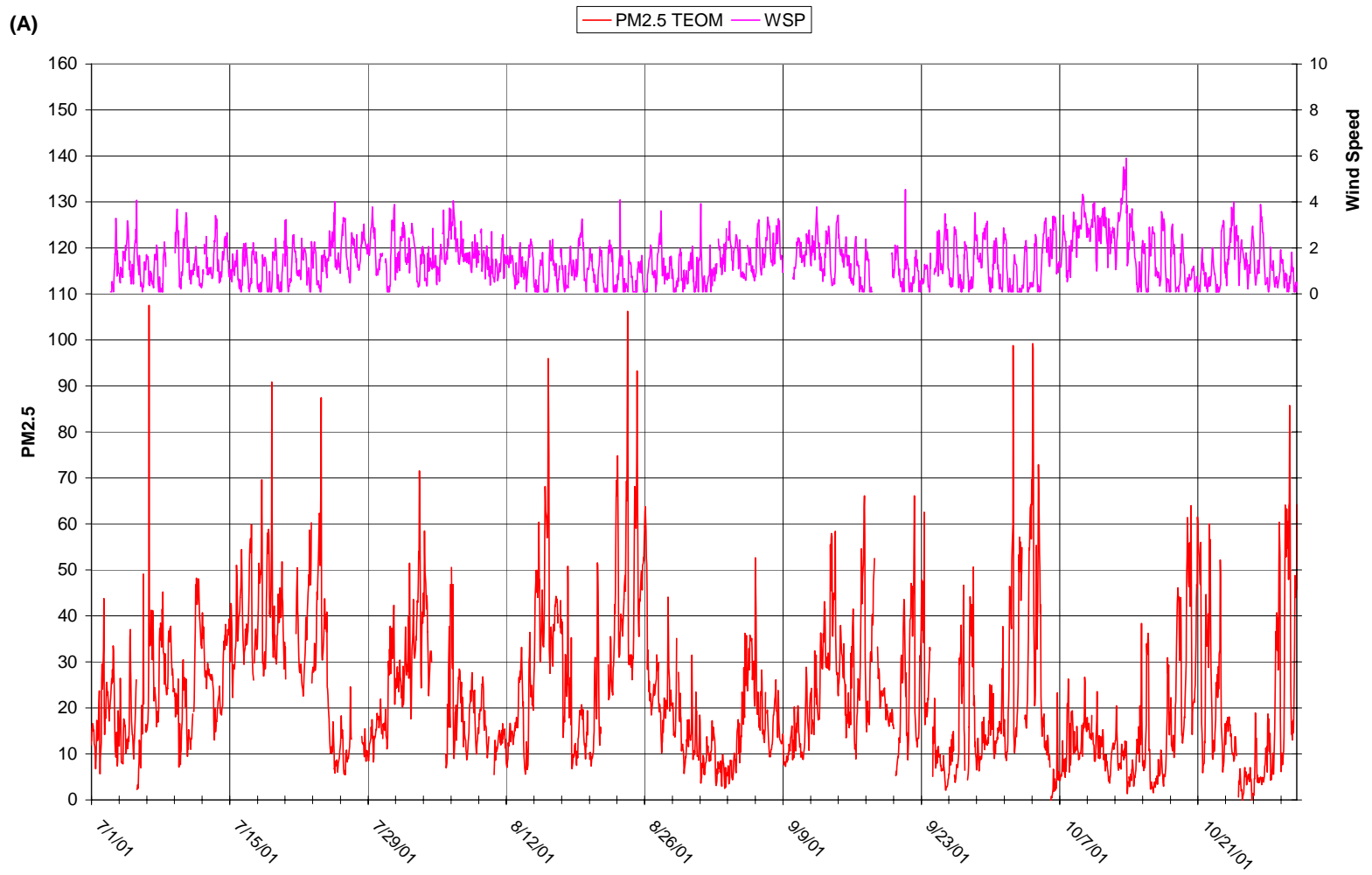

(B)
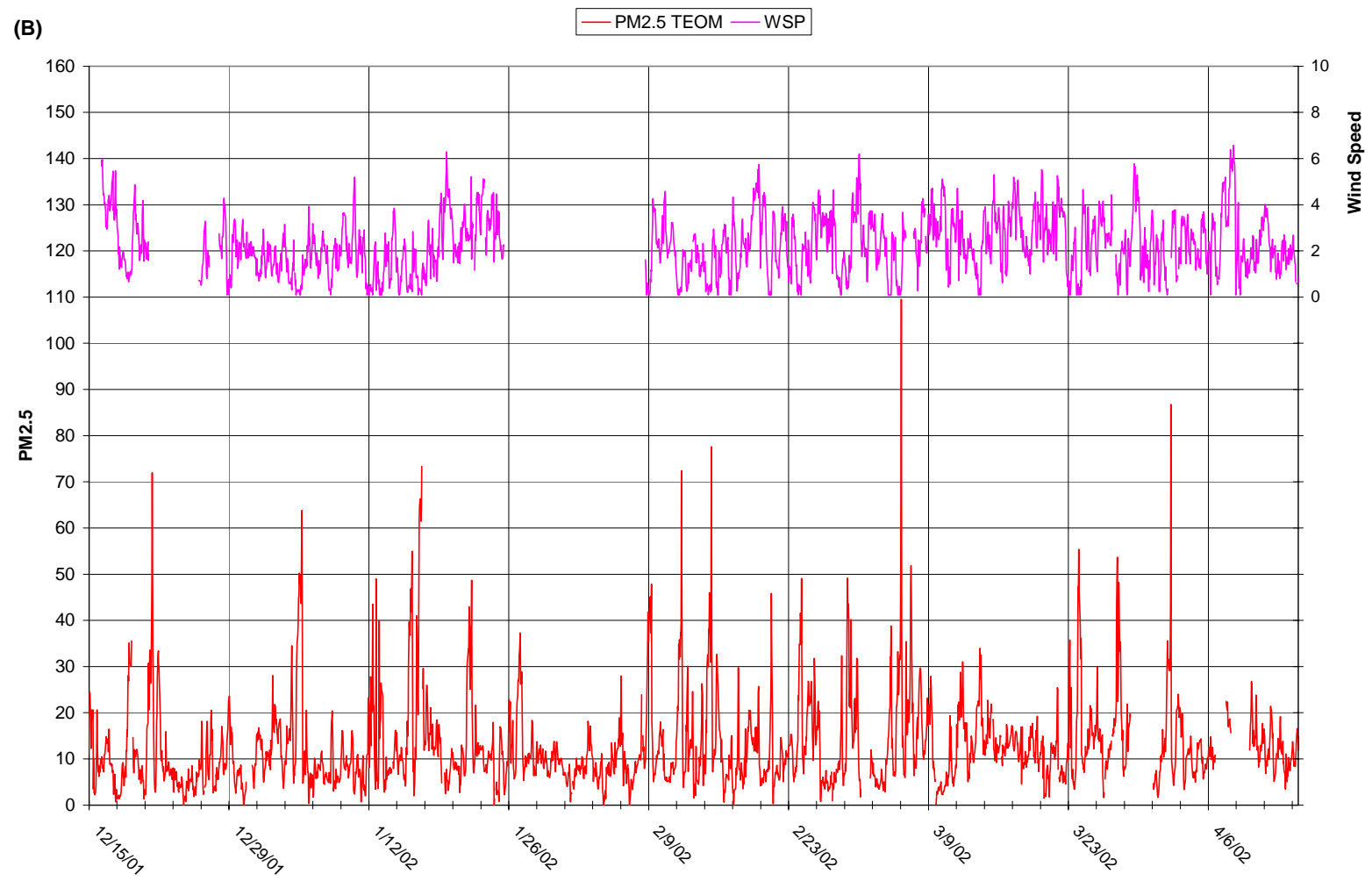

Figure 17. Hourly average TEOM $\mathrm{PM}_{2.5}$ concentration and wind speed over four-month periods: (A) 7/1/01 - 10/31/01 summer; (B) 12/15/01 - 4/15/02 winter. 
Figure 17B continues the plot of the $\mathrm{PM}_{2.5}$ and wind data over the four month period beginning $12 / 16 / 2001$. This winter period contains the lowest monthly $\mathrm{PM}_{2.5}$ concentrations of the study, in contrast to Figure 17A, which contained the highest monthly means. The two figures are plotted on the same scale for comparison. The time variation in the four-month winter period shown in the figure has some notable differences from the earlier summer data. Multiday clusters of elevated $\mathrm{PM}_{2.5}$ concentration, to the extent that they can be seen at all, are shorter, only 2-3 days in length. While several days do occur with high $\left(>60 \mu \mathrm{g} / \mathrm{m}^{3}\right)$ peak hourly concentrations, they are fewer in number and not consecutive. Daily minimum values generally fall below $10 \mu \mathrm{g} / \mathrm{m}^{3}$, in contrast with Figure17A, which has several multiday periods having no hourly concentration below $15 \mu \mathrm{g} / \mathrm{m}^{3}$. The $\mathrm{PM}_{2.5}$ time pattern is also reflected in the different of wind speeds in the figure, which corresponds to the difference in seasonal meteorology in this region. The winter weather in Birmingham is typically driven by recurring polar frontal systems which arrive from the Midwest, punctuated by parcels of tropical maritime flow from the Gulf of Mexico. The atmosphere is less stable overall, without the multiday stagnant episodes common to the summer months. Accordingly, the wind speeds plotted in the figure are higher overall, with few days having overnight wind averages under $0.3 \mathrm{~m} / \mathrm{s}$. Not surprisingly, these days generally correspond to those mentioned above with higher peak $\mathrm{PM}_{2.5}$ concentrations.

Figures 18 and 19 show further expanded portions of the data in Figures17A and B, still presented at hourly resolution. Figure 18 contains a two week period of Figure 17A that spans the $\mathrm{PM}_{2.5}$ "episode" of 8/21-26/2001 and the less active period before the next episode. The figure also contains hourly values of mean wind speed and wind direction. At this scale some typical features of both diurnal and multiday cycles can be seen. The first recurring feature is a peak in the $\mathrm{PM}_{2.5}$ concentration in the early morning, in this figure for hours beginning at either 6 or 7 AM. This feature is seen in all PM size measures (including $\mathrm{PM}_{2.5}, \mathrm{PM}_{10}$, ultrafine PM, particle number), and over all time periods. In addition to the morning peak, the general daily time pattern here is repeated on summer days with elevated $\mathrm{PM}_{2.5}$ concentrations. As will be described further below, the morning peak occurs typically about an hour after sunrise, and with time that tracks accordingly over the course of the year. While on some days this morning peak is distorted by other features (e.g., 8/26/01) or apparently absent (e.g., 8/19/01), it is generally the most obvious feature of the daily time sequence, and usually represents the daily maximum concentration, especially on "episode” days. The morning peak is typically about $2 \mathrm{hr}$ in duration, followed by a rapid drop in concentration to a minimum during the remaining daytime hours, followed by a rise in concentration in the evening leading to the next day's morning peak. The time course of the evening rise in concentration is variable and corresponds loosely with the wind speed time pattern, beginning several hours after the winds have calmed. Frequently the concentration buildup proceeds slowly through the daylight hours and reaches a plateau in the evening or early morning hours, followed by the sharp rise of the morning peak. Often there is secondary structure or earlier peaks in this overnight period, as is seen in the figure. For the time of this plot, these peaks are minor in comparison to the morning peak. 


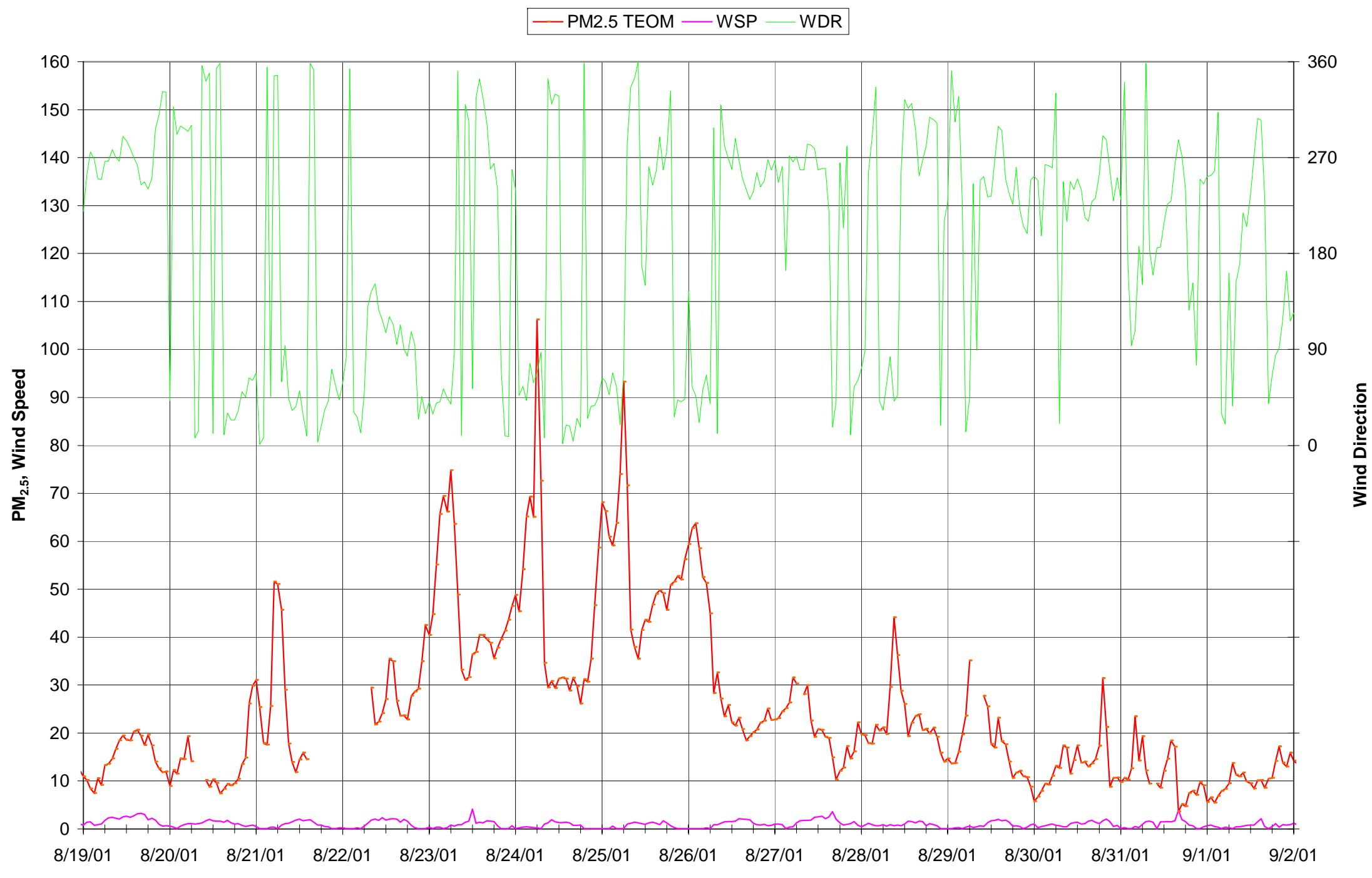

Figure 18. Hourly average TEOM $\mathrm{PM}_{2.5}$ concentration, wind speed and direction over two week period beginning 8/19/01. 


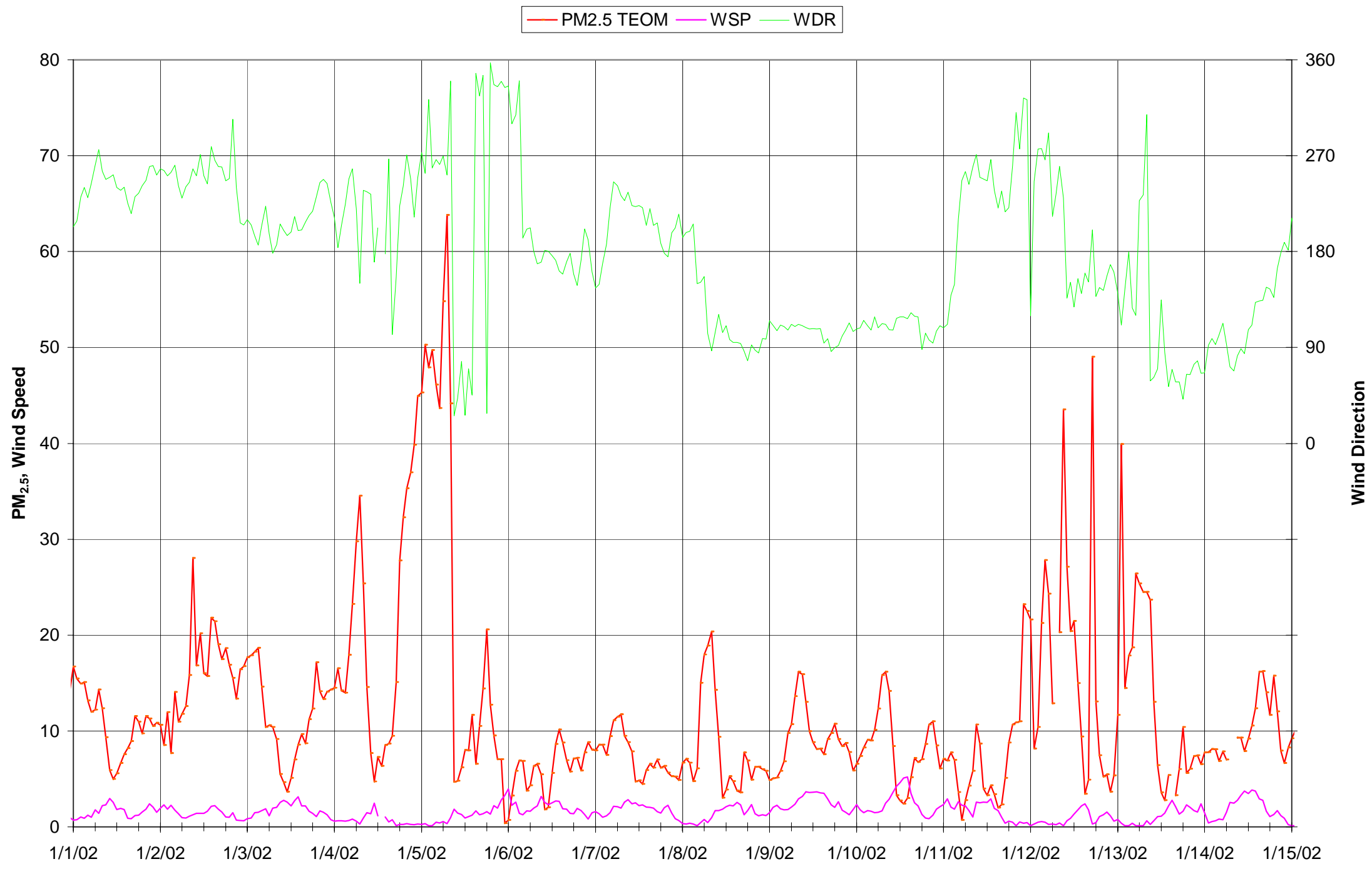

Figure 19. Hourly average TEOM $\mathrm{PM}_{2.5}$ concentration, wind speed and direction over two week period beginning 1/1/02. 
Figure 19 contains a corresponding two week period beginning on January 1, 2002. The daily time series in this figure, and during the winter period in general, are more variable than the summer pattern seen in the previous figure. The morning peak, which falls at 8 or 9 AM in this figure, is still visible on most days, but does not dominate the time series, especially on the majority of days which do not have peak $\mathrm{PM}_{2.5}$ concentrations above $30 \mu \mathrm{g} / \mathrm{m}^{3}$. On these days the relative contribution of other peaks is equal or greater than that of the smaller morning peak. There are several of these peaks in the figure, occurring at different times of the day, often coincident with changes in wind direction. These appear to be the result of plume transit across the site, though from this figure it would be difficult to assign directions to the source or sources responsible for most peaks.

The time scale is expanded once more in Figures 20, 21A and 21B, which present the data from several instruments at the full time resolution used in this study In addition to the SRI PM 2.5 TEOM, the plots show the output of the nephelometer, APS, and the $\mathrm{PM}_{10}$ TEOM operated by JCHD, all obtained at one minute intervals. For reference, the one-hour average $\mathrm{PM}_{2.5}$ and $\mathrm{PM}_{10}$ TEOM output data from the JCHD instruments as reported on the EPA AIRS database is also included. Figure 20 contains the data from these instruments for the three day period beginning on $8 / 23 / 2001$. The time pattern seen by all instruments is qualitatively the same, following the "summer episode" pattern described above. At the enhanced time resolution, the structure in the time series including the morning peak is more evident, as is the overnight plateau feature typified by the evening of $8 / 24$. This structure allows some insight into the question of the optimum instrumental time resolution. Clearly the fine structure in the time series data occurs on sub-hourly time scales, and the width of many of the fine peaks from both TEOMs is 15-30 minutes. A few features even shorter than this were detected by the APS and nephelometer, which are both able to operate with time response corresponding to the one minute data output rate. These include the peak at 8/24/01 4:33AM (4-5 minutes FWHM) and 8/25/01 11:40 AM ( $<3$ minutes FWHM). Such events are rare, and must correspond to a transient plume impact from a nearby source. In general, it seems that while the one minute data set obtained for this study will have some benefits in separating and assessing the impacts of sources with defined plumes at the measurement site, hourly average time resolution will be generally adequate to represent the daily time trace data. Figure 21A shows data from the same instruments over a 48 hr period during January 2002. Again, all instruments show similar patterns, including structure that is obscured in the hourly average time series plot of Figure 19. Figure 21B shows the wind speed and direction during this time period, as well as the outputs from the 8400S sulfate monitor, which were not included in the previous figures. The local winds were light and variable over this period, and most of the particulate peaks correspond to times when the wind was stagnant or shifting in direction. These days represent a typical winter pm episode at the site: after the passage of a frontal system and rain event, the atmosphere was stable, with overcast skies and low mixing depth, which apparently trapped the emissions of the local sources close to the site into meandering plumes which impacted the samplers repeatedly over the two day period. 


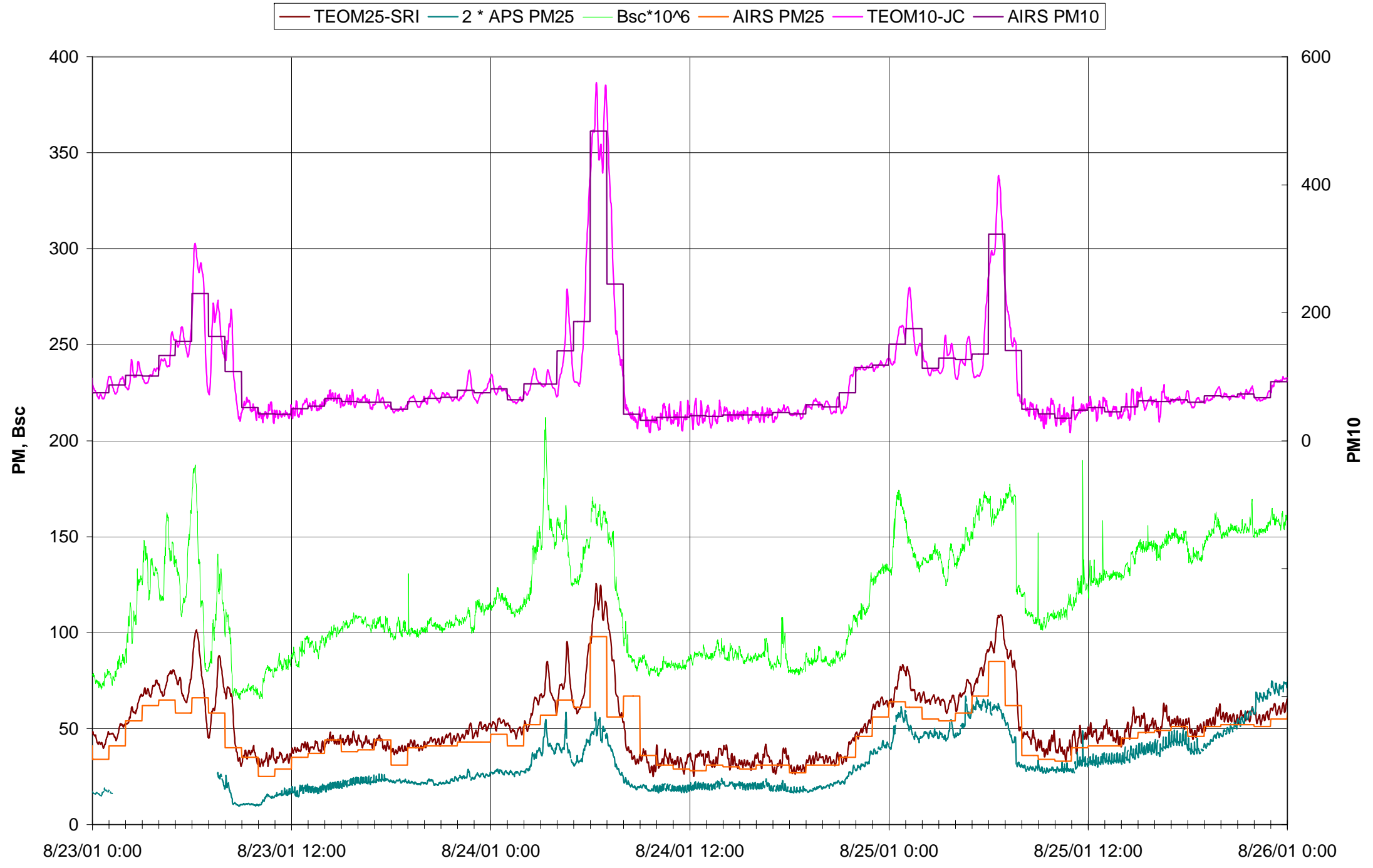

Figure 20. PM data from several instruments for 72 hour period beginning noon, 8/23/01. 
(A)

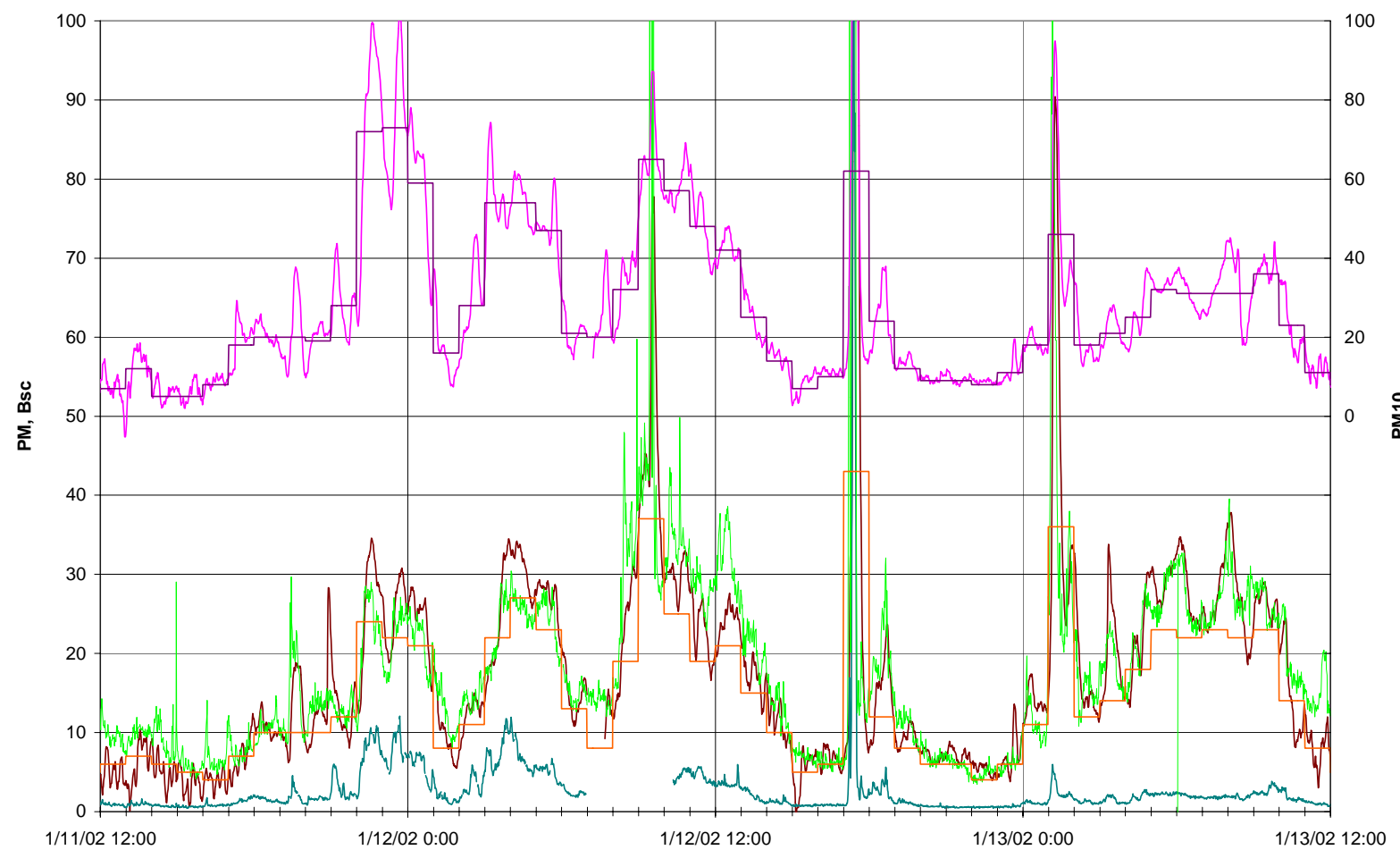

(B)

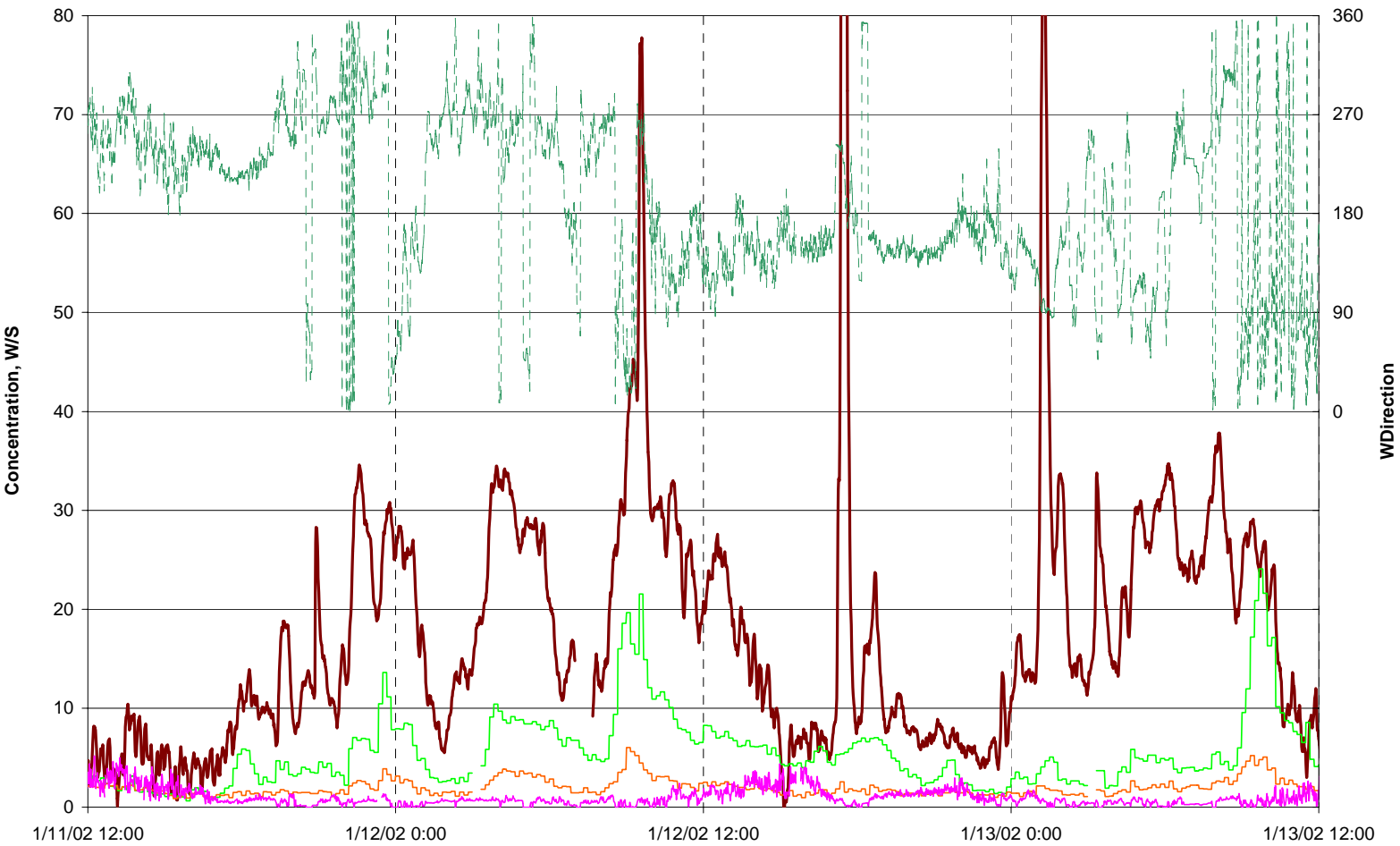

Figure 21. Real-time PM instrumental data for 48 hour period beginning noon, 1/11/02: (A) minute and hour average data; (B) TEOM, 8400S, and wind data. 
The 8400S data included in Figure 21B illustrate another pattern typical of the entire data period: neither of the two outputs track the $\mathrm{PM}_{2.5}$ monitor as closely as any of the instruments in the previous figure. The $\mathrm{PM}_{2.5}$ sulfate concentration appears to follow the broad trend of the total $\mathrm{PM}_{2.5}$ mass seen by the TEOM, but has little response to the material in the three largest peaks in the figure. The gaseous $\mathrm{SO}_{2}$ time series, on the other hand, shows peaks of its own, which are coincident with some but not most of the $\mathrm{PM}_{2.5}$ peaks. This suggests that some of the local $\mathrm{PM}_{2.5}$ sources (such as the two nearby coke generation facilities) may also be sources of $\mathrm{SO}_{2}$ and possibly of primary particulate sulfate as well. The $\mathrm{SO}_{2}$ and sulfate time traces clearly differ from the TEOM in the behavior of the morning peak. While the particulate sulfate often does increase at the time of the TEOM morning peak, the increase in sulfate is less consistent and proportionally much smaller than that of total particulate mass. Gaseous $\mathrm{SO}_{2}$, in contrast, frequently exhibits a pronounced prominent peak in the morning, but the time of day of this peak is less consistent, but generally occurs an hour or more after the particulate morning peak. The peak in Figure 21B at around $10 \mathrm{AM}$ on 1/13/02 is an example of this behavior.

\section{$\underline{\text { Seasonal }}$}

As described in the discussion of Figures 14 and 15, the data in this study suggest an underlying seasonal pattern in the average $\mathrm{PM}_{2.5}$ concentrations at the site, though month to month variations limit the conclusions possible from an 18 month study confined to portions of two calendar years. To add a longer-term perspective, the $\mathrm{PM}_{2.5}$ data from this study are plotted in Figure 22 in the context of four years of data taken at the site by JCHD and reported on the EPA AIRS database. The AIRS data in Figure 22A include monthly average concentrations measured by the FRM PM $\mathrm{PM}_{2.5}$ and TEOM PM 10 for the full 2001-2004 calendar years, and from the JCHD $\mathrm{PM}_{2.5}$ TEOM after its initial deployment in July, 2001. The figure also contains estimated PMc computed from the difference of the $\mathrm{PM}_{10}$ and $\mathrm{PM}_{2.5}$ TEOM averages. The same data are shown as month of year composite averages in Figure 22B, where the average for each device is computed over the months of its operation in Figure 22A. The four year data set gives stronger indication of a recurring seasonal pattern superimposed on a monotonic yearly decrease in ambient particulate concentrations. This pattern includes high concentrations in late summer and early fall, low concentrations in January and February, and intermediate concentrations in the spring, with suggestions of a secondary maximum in April or May. The $\mathrm{PM}_{2.5}$ during the project period (indicated by the SRI TEOM average in Figure 22B) follows the four-year pattern indicated by the other two $\mathrm{PM}_{2.5}$ monitors in the figure) except for the month of November, 2001, which had atypically high $\mathrm{PM}_{2.5}$ and the highest monthly $\mathrm{PM}_{10}$ of the entire four year period.

The seasonal pattern in the calculated PMc over the four year period is similar in general to the $\mathrm{PM}_{2.5}$ pattern. Monthly average PMc is typically 3-5 $\mu \mathrm{g} / \mathrm{m}^{3}$ lower than average $\mathrm{PM}_{2.5}$, especially in the winter months when both size fractions are lowest; there are several months, however, with higher PMc than $\mathrm{PM}_{2.5}$; these months occur in the fall or during the April- May periods that track higher for both size fractions. 
(A)

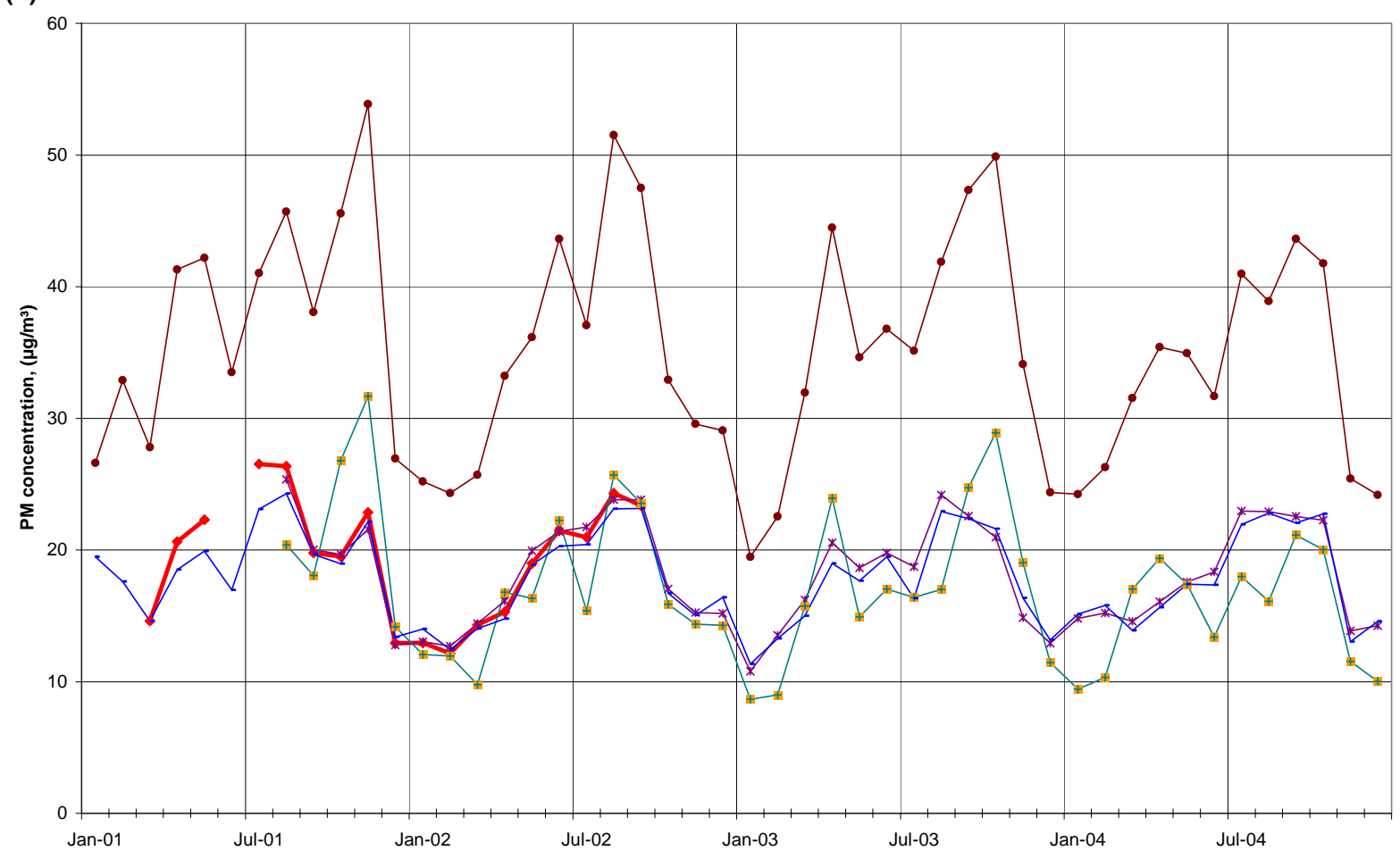

(B) $\rightarrow$ SRI TEOM ZC $\rightarrow$-AIRS TEOM25 $\rightarrow$ AIRS TEOM10 $\rightarrow$-AIRS TEOM PMc $\rightarrow$ AIRS FRM PM25

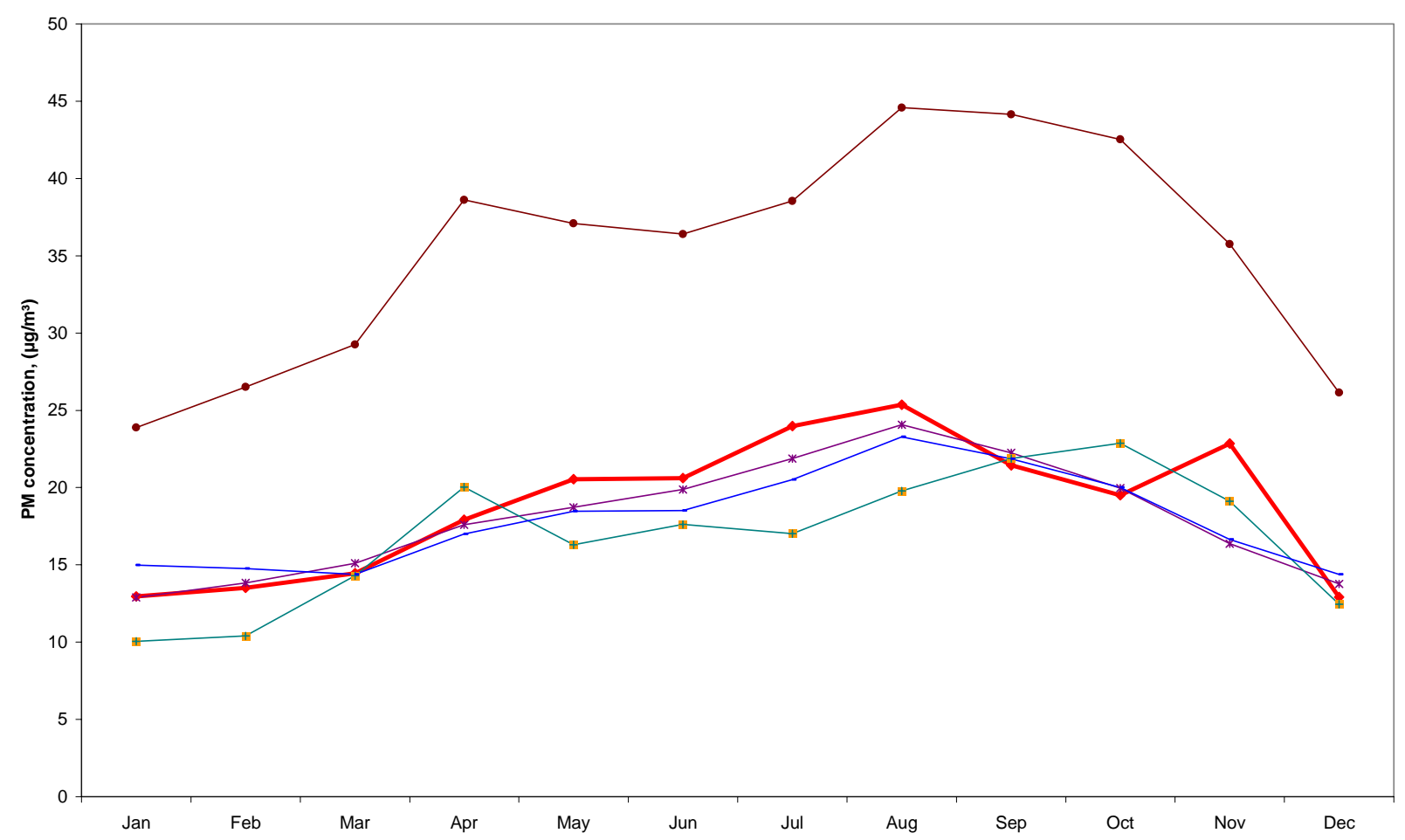

Figure 22. Monthly average project period TEOM PM 2.5 in context of 2001-04 AIRS PM $_{2.5}$, PM$_{10}$, and PMc data: (A) by month; (B) by month of year. 


\section{Day of week}

As described in the introductory paragraphs of this section, the time variation of ambient $\mathrm{PM}_{2.5}$ includes both irregular multiday cycles on the order of 3-8 days (which follow synoptic weather patterns) and systematic weekly cycles that follow regular variations in source emissions. Emissions-related concentration patterns are expected to show reduced concentrations of affected pollutants on weekend days, when mobile source activity is reduced, as is production activity at many stationary sources. This emission pattern is well documented for mobile sources in the Birmingham area as well as other urban areas. Our observation of stack plumes from point sources in the area suggests that several of these operate on five- or six-day production cycles as well. Both irregular and systematic weekly cycles are seen in the data from this project, and are not clearly distinguished in a visual presentation of the data. For example, the time series plots in Figures 17-19 show repeated clusters of elevated $\mathrm{PM}_{2.5}$ approximately a week in length, but the days with highest average $\mathrm{PM}_{2.5}$ in Figures 18-19 (8/25/01 and 1/5/02) are both Saturdays, which would be expected to have lower $\mathrm{PM}_{2.5}$ (as is the case, on average, over the project period).

In this section, systematic weekly cycles are assessed through collective "day of week" averages of instrument output data over the entire project period, if possible, or over the largest portion of the project period where applicable data are available. These averages were constructed from the set of valid hourly averages. For SRI data, the hourly averages are averages of shorter-term measurements free of error flags covering at least 54 minutes of one-minute data, $55 \mathrm{~min}$ of SMPS data, or 50 minutes of 8400 s cycle data). Hourly published AIRS or ARA data are used directly if unflagged. Figures 23-28 in this section contain plots day of week averages of several pollutants, as well as relative day of week averages, defined as the ratio of each day of week average to the overall period average of that pollutant. As a figure of merit for the relative effect of reduced weekend emissions, we also calculate a Work Week Ratio (WWR) for each pollutant. The WWR is the ratio of the mean of the Monday through Friday day of week concentrations to the mean of the Saturday and Sunday day of week concentrations. To first order, the WWR and the relative day of week averages for different pollutants may be compared to indicate differences in source contributions for the pollutants. Such comparisons, however, are strictly valid only if the data are averaged over the same time periods, which was not always the case. The discussion of the figures in this section addresses specific instances where the differing operating periods of some instruments is a consideration.

Day of week averages were also computed with a five hour time shift; that is, assigning a measurement to a day if it falls between 5:00 AM on that day and 4:59 AM the next day. The shifted day of week calculation has the effect of including the early morning concentrations of a pollutant with the previous day when the pollutant was probably emitted, but does not reassign the elevated concentrations during the morning peak. For most pollutants this calculation has the effect of increasing the Monday average concentration at the expense of the Saturday average (median change $4 \%$ for each), with smaller changes for other days, resulting in a median decrease of 3.3\% to the WWR when the shifted averages are used. The time-shifted calculations are obviously inapplicable to 24 hour filter measurements such as the FRM or speciation monitors which collect samples integrated over a fixed calendar day. 

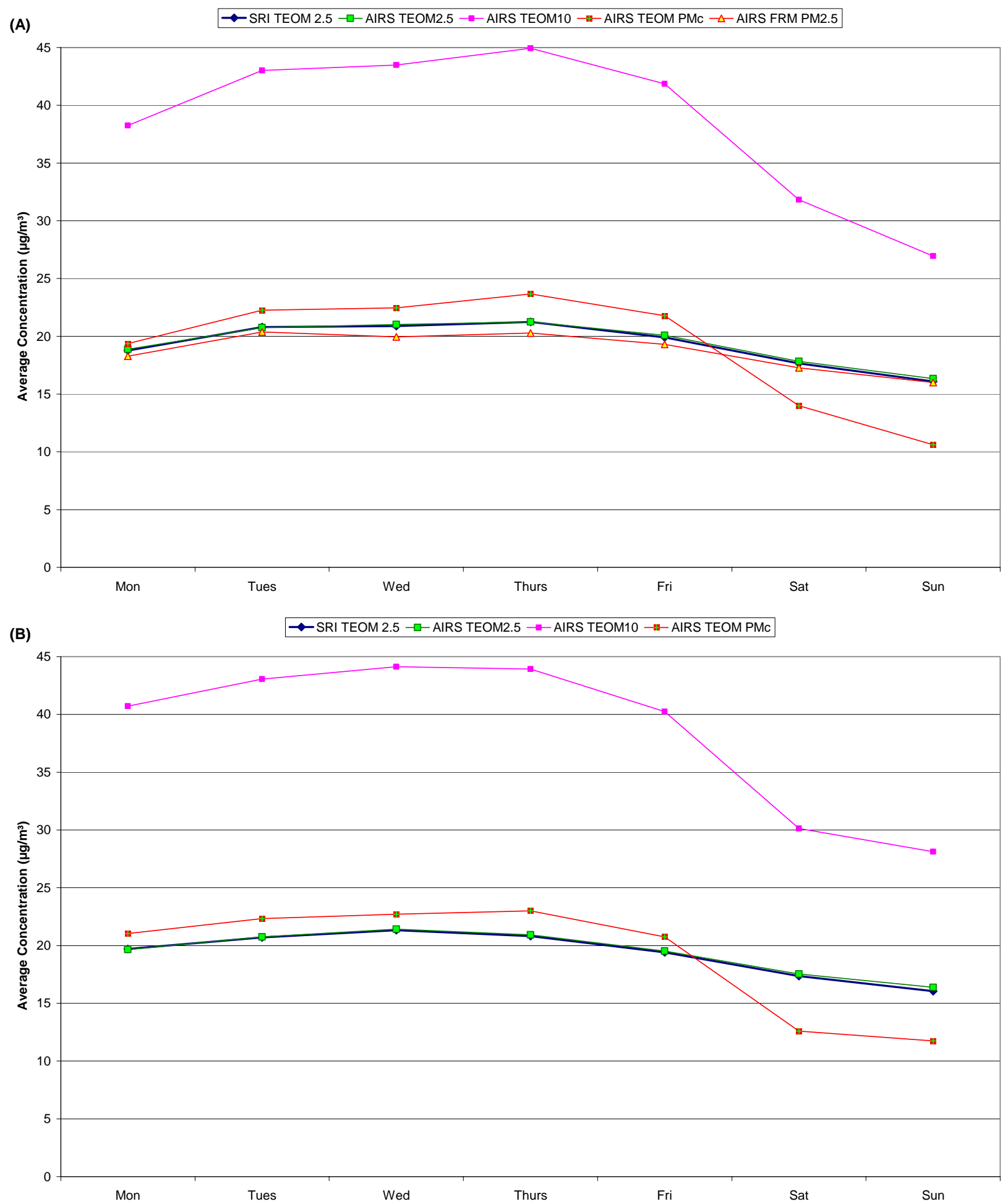

Figure 23. Day of week average SRI TEOM PM $\mathbf{P M}_{2.5}$ with AIRS $\mathbf{P M}_{2.5}, \mathbf{P M}_{10}$, and PMc data: (A) midnight to midnight; (B) starting at 5:00AM each day. 
Figure 23A presents day of week average concentrations measured by TEOM and FRM monitors at the site during the period of the project. The averages were taken over the 9158 hours during the project period for which valid data exist for all four of the instruments. In the figure the three $\mathrm{PM}_{2.5}$ instruments follow a common pattern: the highest daily averages fall on Tuesday through Thursday at approximately $20-21 \mu \mathrm{g} / \mathrm{m}^{3}$. Below this, in decreasing steps of about $1 \mu \mathrm{g} / \mathrm{m}^{3}$, fall averages for Friday, Monday, and Saturday, followed by Sunday averages slightly over 16 $\mu \mathrm{g} / \mathrm{m}^{3}$. The $\mathrm{PM}_{10}$ TEOM averages follow the same general order, but the falloff of the lowest three daily averages is more pronounced. Accordingly, the weekend deficit becomes more notable in the order $\mathrm{PM}_{2.5}<\mathrm{PM}_{10}<\mathrm{PMc}$. Figure 23B shows the same data when averaged with a five hour time shift as described above. The FRM data are omitted from this figure for the reason discussed there. After the time adjustment, Monday averages for all instruments are increased to approximately the Friday levels, while the Saturday concentrations drop closer to the Sunday levels. Work Week Ratios for $\mathrm{PM}_{2.5}, \mathrm{PM}_{10}$, and PMc, respectively, are 1.22 (1.21 for the AIRS $\mathrm{PM}_{2.5}$ TEOM), 1.46, and 1.80.

The day of week trends seen for $\mathrm{PM}_{2.5}$ in Figures 23A and 23B provide a reference for the weekly patterns followed by other pollutants or PM related quantities. This prototype weekly trend has comparable values for the work week which are higher than weekend days by a factor that can range from less than 1.1 to almost 2. Within these groups Monday averages may be lower (even for the time shifted calculations) and Saturday is generally higher than Sunday. This general pattern would be expected for a locally generated pollutant with atmospheric lifetime on the order of 12-24 hours and generation rates that are roughly the same on the normal work week days, reduced on Saturday and further reduced on Sunday. As described earlier, this emission pattern is observed for a range of pollutants from both mobile and stationary sources in the Birmingham area as well as other urban areas. The day of week trends and especially the weekend reductions are more clearly visible in Figure 24, which presents the data of Figure 23B as relative day of week averages. In addition, the figure contains size-integrated concentrations from the SMPS and APS that most closely correspond to $\mathrm{PM}_{2.5}$ for each instrument. These averages are not computed over the same time period as the other instruments, but the day of week averages do qualitatively track the prototype weekly pattern of the $\mathrm{PM}_{2.5}$ TEOMs.

Figures 25-28 present relative day of week average data from other instruments at the site in formats that allow comparison of the weekly trends for different pollutants or particulate fractions. The SRI PM 2.5 TEOM data are plotted in each figure for comparison. Since the data sets in these figures are reduced to hours contemporaneous to the operation of the specific instruments in each figure, the $\mathrm{PM}_{2.5}$ TEOM averages vary somewhat from figure to figure while following the prototype pattern described above. All averages incorporate the five hour time shift, which we judge more representative for calculations of WWR.

Figure 25 contains data from the Radiance 904 Nephelometer and R\&P 8400S sulfate monitor averaged over the 8688 common hours of operation of these two instruments and the TEOM. The nephelometer Bsc averages are less variable than the $\mathrm{PM}_{2.5}$, with a clear weekend deficit only on Sunday (WWR $=1.05$ as compared to 1.18 for the TEOM over this time frame). In contrast, the $\mathrm{PM}_{2.5}$ sulfate measured by the 8400 S tracks the day of week trend of the TEOM more closely during the work week, but with a weekend deficit roughly half that of the total $\mathrm{PM}_{2.5}$ measurement (WWR $\left.=1.10\right)$. Ambient $\mathrm{SO}_{2}$ concentrations, which are an auxiliary output of the $8400 \mathrm{~S}$ monitor, show a weekly pattern notably different from any of the other pollutants monitored at the site. During the work week, average concentrations are lowest on Monday, then 
peak on Tuesday about 15\% higher than the levels on the next three days, followed by a weekend deficit (WWR = 1.13) only slightly less than that of the $\mathrm{PM}_{2.5}$ concentration. The figure also shows $\mathrm{SO}_{2}$ data from the ARA gas monitor at the site (averaged over the project period but not over the same hours), which shows the same Tuesday peak as the $8400 \mathrm{~S} \mathrm{SO}_{2}$ measurement. Survey of both data sets shows the elevated Tuesday pattern to be recurring, rather than the artifact of one or two high days measured by either device. While we infer that this pattern reflects the operating pattern of one or more local emissions sources, we have no specific information to identify the sources responsible.

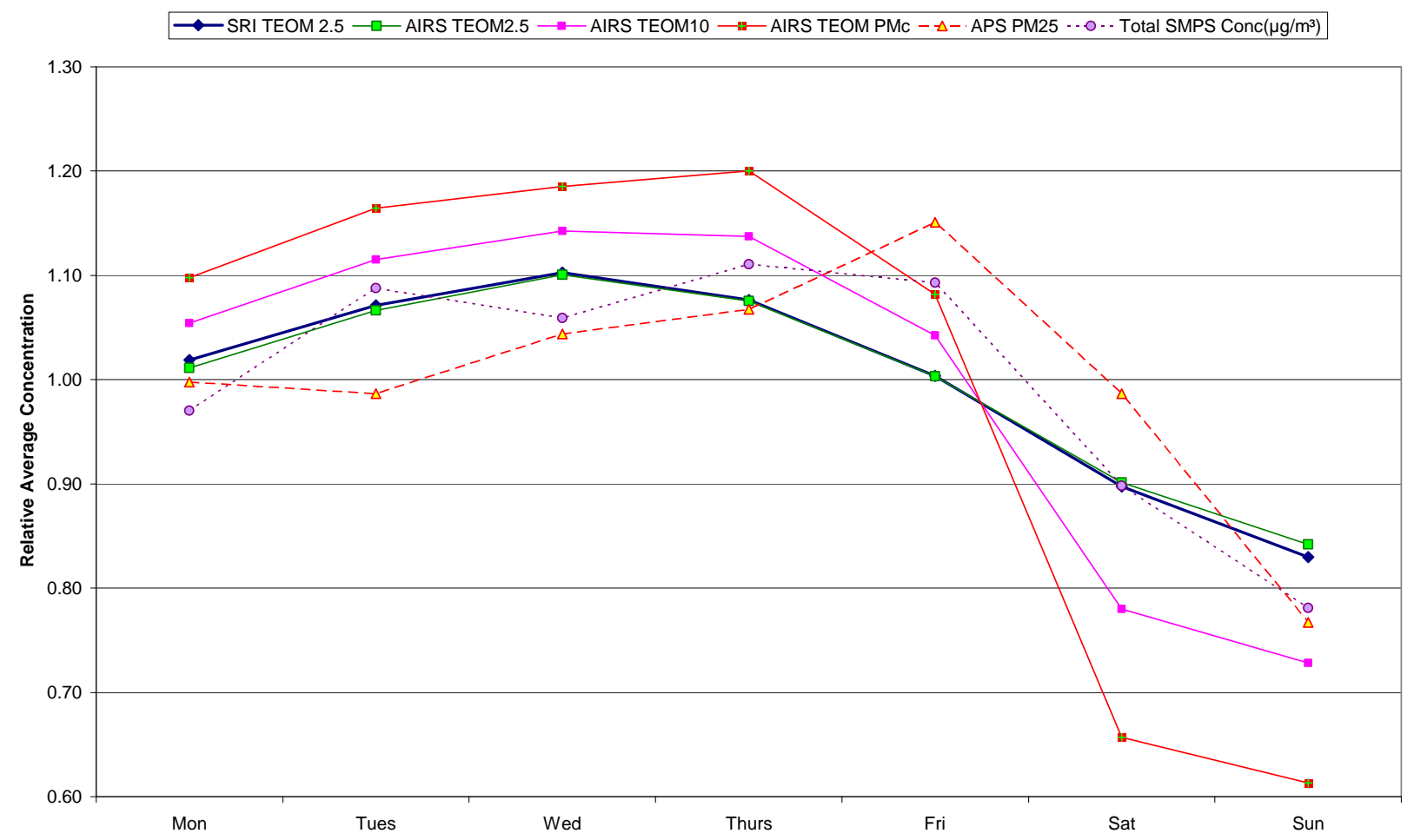

Figure 24. Day of week relative average $\mathrm{PM}_{2.5}, \mathrm{PM}_{10}$, and $\mathrm{PMc}$ data starting at 5:00AM each day.

Figure 26 contains data from the TSI 3496 SMPS averaged over the 7407 common hours of operation of this instrument and the SRI TEOM (of 8042 complete SMPS hours during the period). Shifted relative day of week averages are shown for several indicator size fractions as well as the TEOM for reference. All of the size fractions follow the general weekly pattern, but seem to fall in two clusters regarding weekend deficit. The total integrated particle mass (volume), total particle number, and integrated particle mass smaller than $100 \mathrm{~nm}\left(\mathrm{PM}_{100}\right)$ have WWR values of $1.23,1.23$, and 1.20 respectively, all comparable to the $1.20 \mathrm{WWR}$ value of the TEOM over this range of hours. The other cluster of particle size groups, with higher weekend deficit, includes the smallest and largest particles measured by the SMPS. This includes the integrated number in the first (N21) or first six (N30) channels of the size spectrum, and the integrated mass-weighted signal for particle diameter greater than $0.5 \mu \mathrm{m}$ (SMPS 0.5-1.0). These size groups, respectively, have WWR $=0.90,1.21$, and 1.46 . 


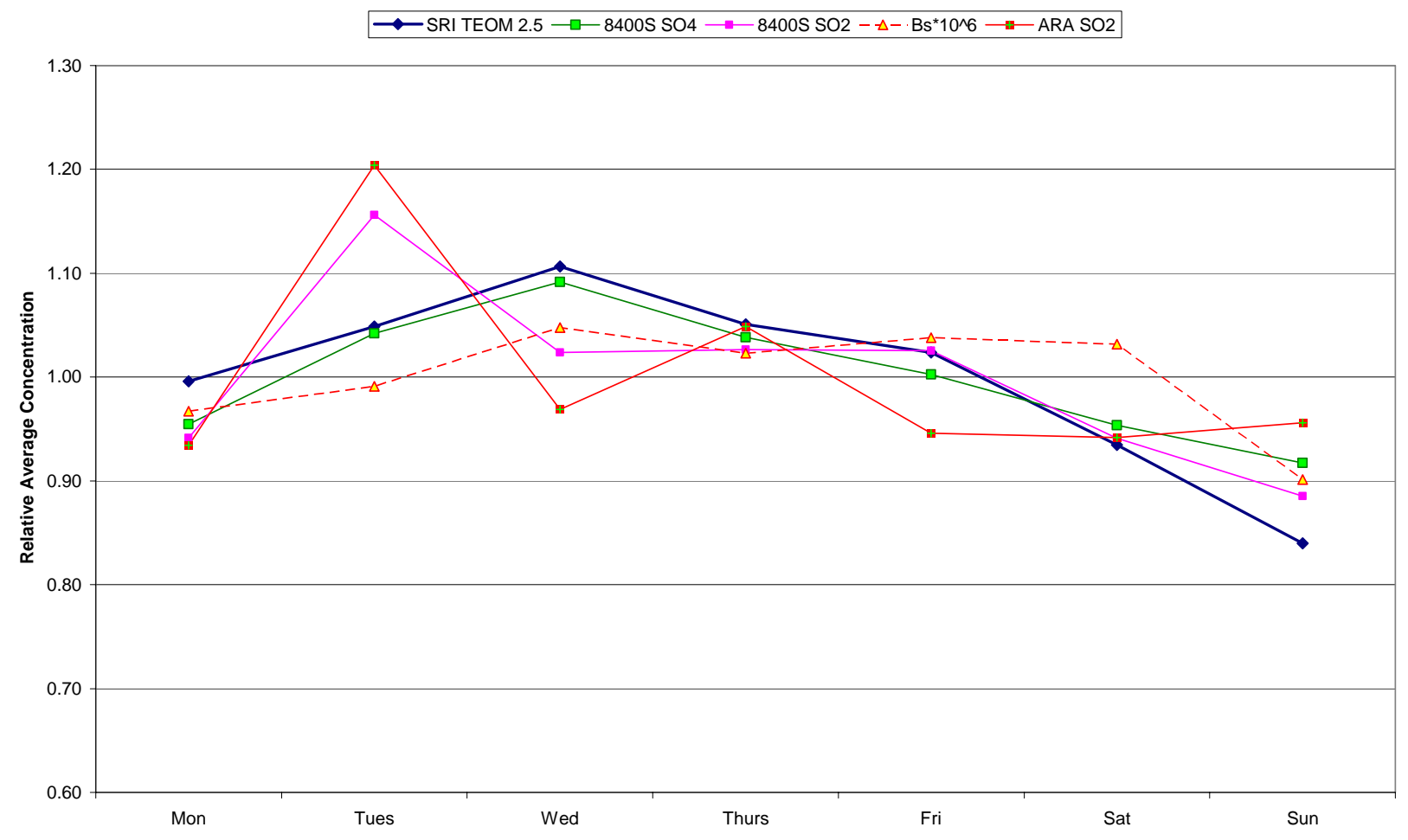

Figure 25. Day of week relative average data from nephelometer and $8400 \mathrm{~S}$ monitor.

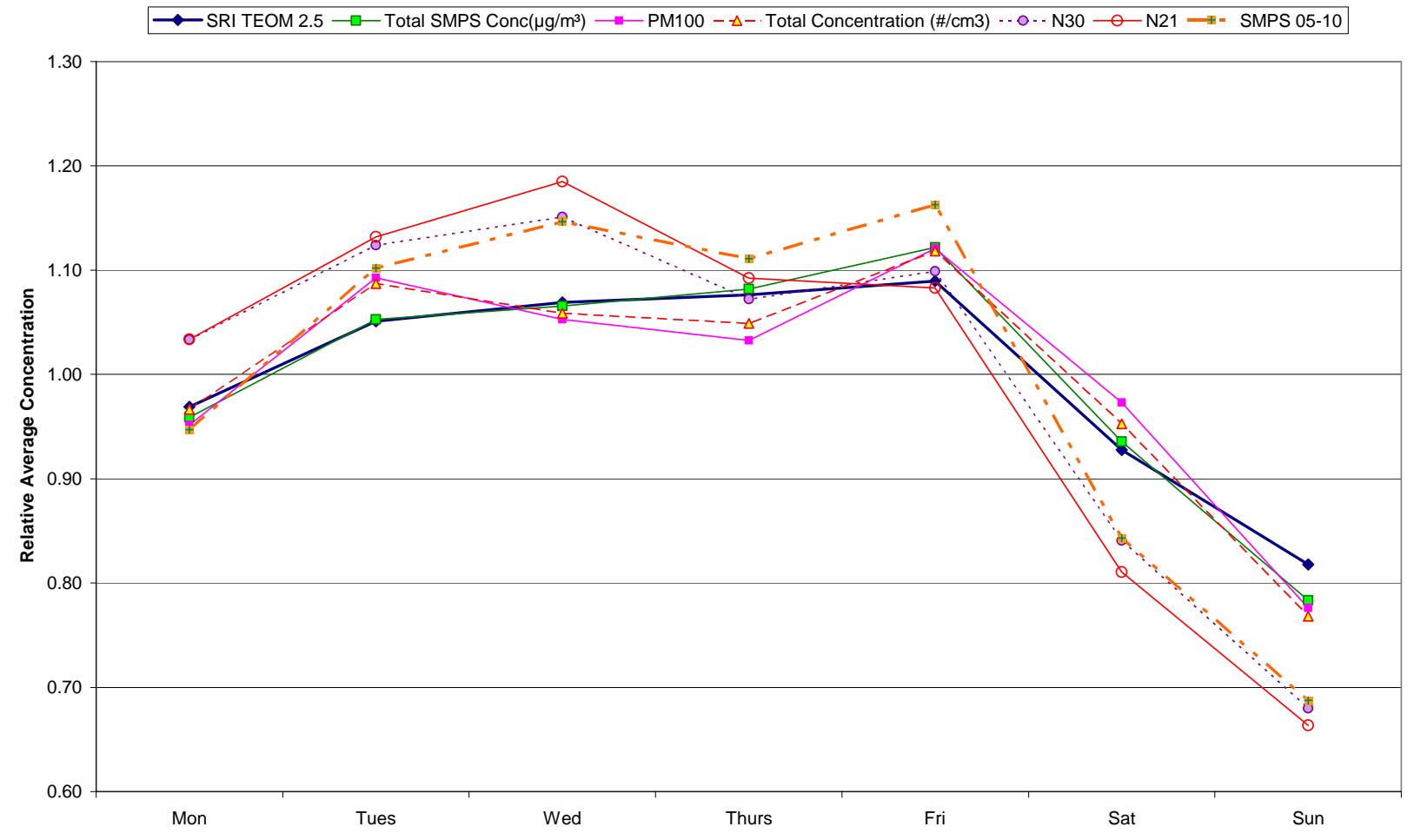

Figure 26. Day of week relative average submicron size fractions from SMPS. 
Figure 27 is an analogous plot using size fractions from the APS 3321 aerodynamic particle sizer. These data should be taken with more caution since the period of operation of this device (2452 hours, primarily during March - August, 2002) is more restricted than the other instruments shown. In addition to the SRI PM 2.5 TEOM, the calculated PMc averages from the AIRS TEOMs are presented for comparison. The figure shows considerable day-to-day scatter in the averages, with a weekend deficit that increases with particle size. WWR values are 1.07, 1.35, and 1.59, respectively, for the size fractions spanning $0.5-1.0 \mu \mathrm{m}, 1.0-2.5 \mu \mathrm{m}$, and $2.5-$ $10 \mu \mathrm{m}$.

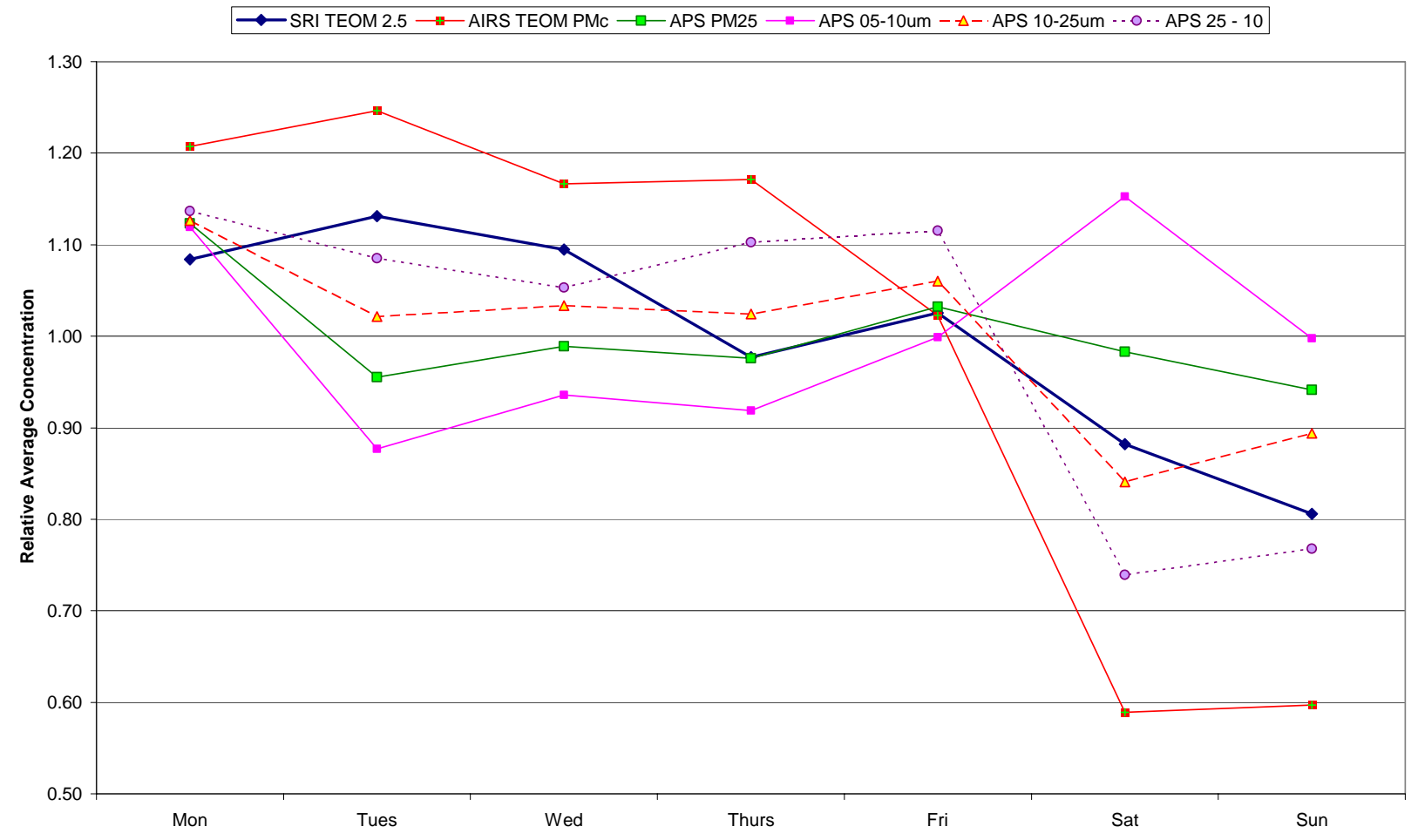

Figure 27. Day of week relative average APS 3321 size fractions (after March, 2002).

Figure 28 contains published ARA data from the onsite continuous gas monitors averaged over 11448 common hours of operation with the SRI TEOM. The number of valid hours for specific instruments varies within the range 10177-10730 hours, except for the ozone monitor (8904 hours). The weekly behavior of the gases varies significantly. The distinct elevated-Tuesday pattern of the $\mathrm{SO}_{2}$ monitor was described above. The weekly $\mathrm{CO}$ pattern most closely follows the prototype $\mathrm{PM}_{2.5}$ trend, with WWR of 1.16. Average NO has a high WWR (1.76) comparable to PMc, and follows a similar day of week pattern. NOy, the atmospheric reaction product of NO, follows a similar but less exaggerated pattern, with a lower WWR of 1.49. On days with high photochemical activity, NOy is a precursor to ozone, but the $\mathrm{O}_{3}$ day of week pattern is dissimilar to those of the nitrogen oxides and has a WWR less than unity $(0.90$, or $10 \%$ higher on weekends). We have no immediate explanation for this counterintuitive result. 


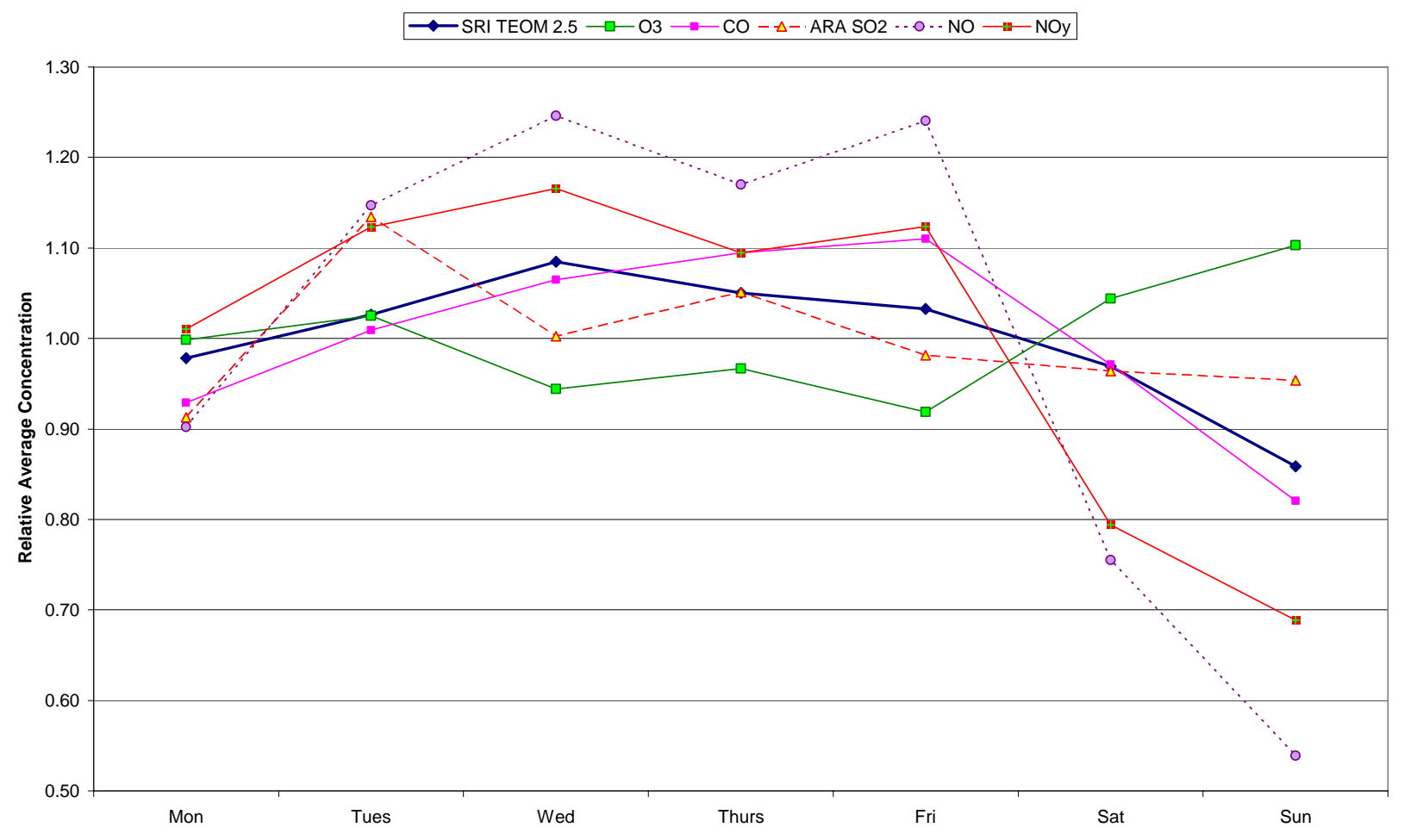

Figure 28. Day of week relative average concentrations from ARA gas monitor data.

\section{Time of day}

While repeating weekly time patterns can safely be attributed to variations in emissions source activity, both emissions and atmospheric factors are responsible for the observed diurnal pattern in pollutant concentrations, and the relative effects of these factors are not easily distinguished. The time pattern for each pollutant results from the interaction of sources and sinks for that pollutant in the local airshed. As described above, the composite source activity for locally generated pollutants generally follows a predictable time pattern for each pollutant, both daily and weekly. Often the emission time patterns are similar, and follow the typical work week. Thus, while the same emissions sources are generally responsible for multiple pollutants; even pollutants generated by a distinct set of sources may have similar emission time profiles. Likewise, the major atmospheric loss mechanism - dilution and dispersion by mixing with ambient air - imposes the same time pattern on multiple pollutants. There is a persistent daily cycle of higher dilution rates in the daylight hours when the average windspeed and mixing depth are higher, and lower dilution losses at night when the mixing depth is lower and wind speeds are reduced or stagnant. On the other hand, different pollutants - including different particle sizes - will have differing rates of change due to atmospheric reactions, photochemical transformations, or other loss mechanisms. The specific result of these source and sink factors is shown in the hour of day average plots shown in Figures $29-33$.

Hour of day averages are constructed from the same set of hourly averages used in the previous section for day of week averages. As in the data shown in Figures 23-28, when comparing the behavior of different pollutants the data averages may be further restricted to common operating times for the instruments involved. 


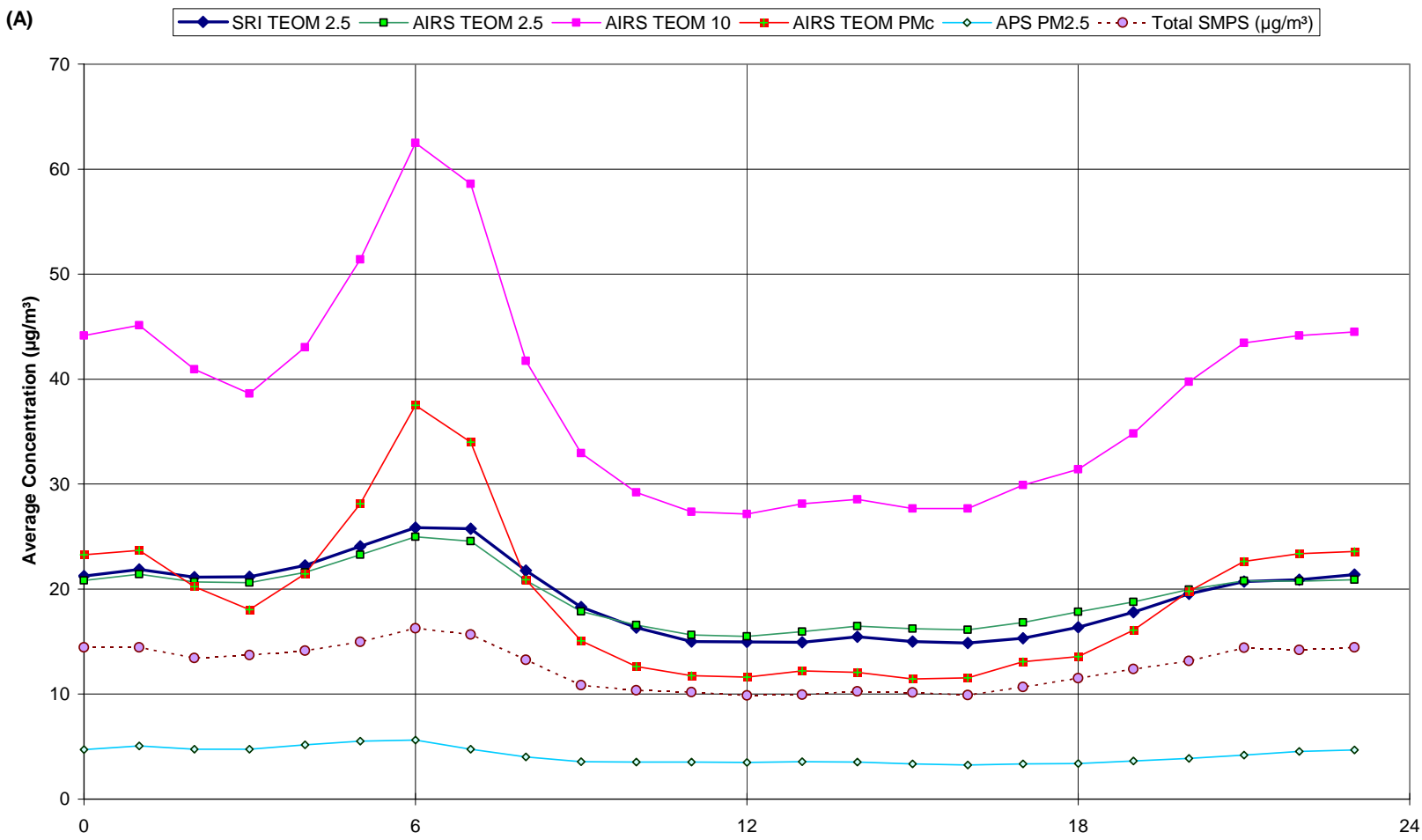

(B) $\longrightarrow$-SRI TEOM $2.5 \multimap \square-$ AIRS TEOM $2.5 \multimap-$ AIRS TEOM $10 \multimap-$ AIRS TEOM PMc $\bullet-$ APS PM2.5 $\cdots 0 \cdots$ Total SMPS $\left(\mu g / m^{3}\right)$

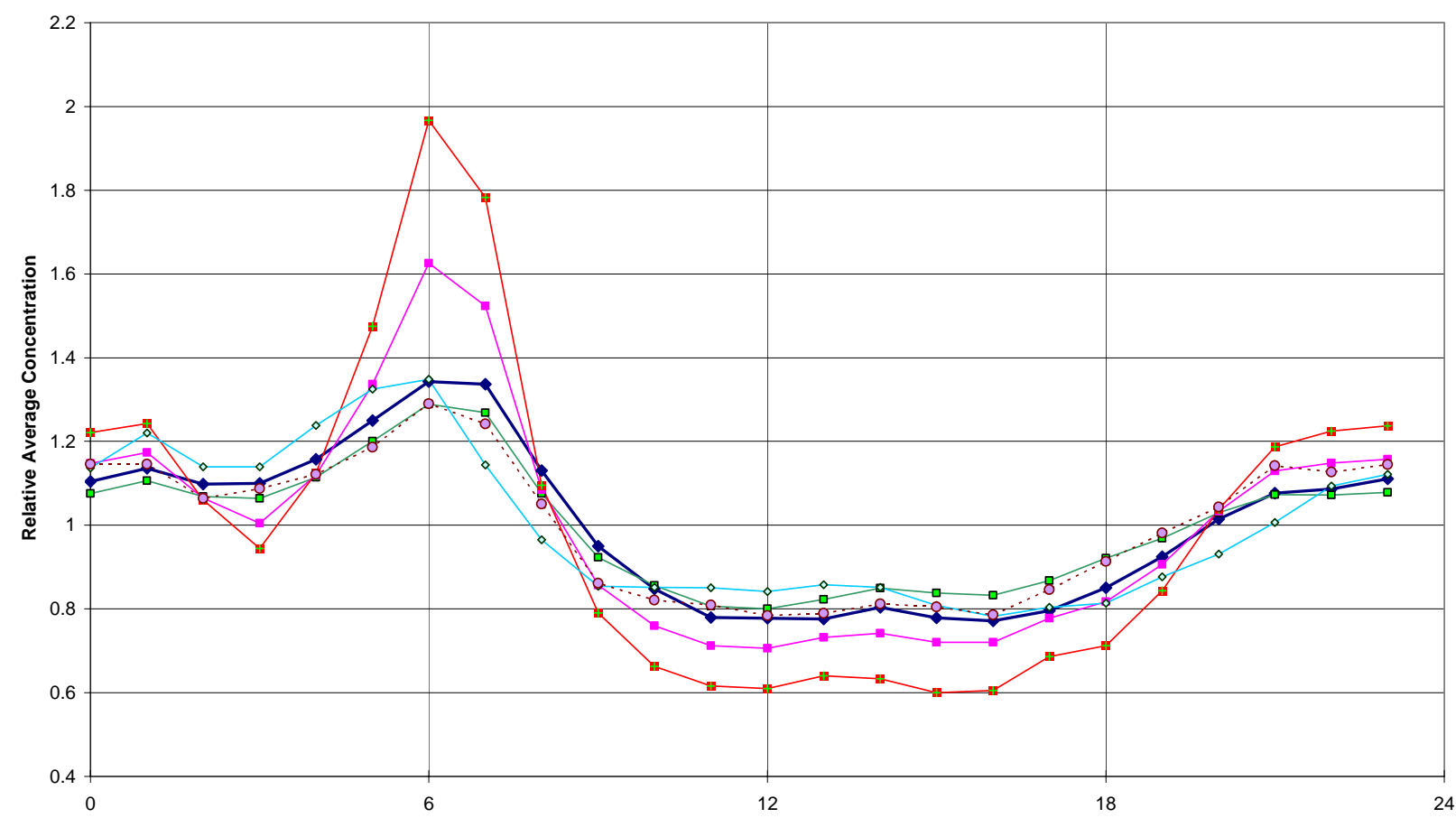

Figure 29. Hour of day average $\mathbf{P M}_{2.5}, \mathbf{P M}_{10}$, and PMc data from several instruments: (A) in mass concentration units; $(B)$ relative to average for each instrument. 
Hour of day average plots for the regulated PM size fractions are presented in concentration units as Figure 29A and in relative units as Figure 29B. These averages cover the 9432 hours of common operation of the three TEOM monitors. Also included are calculated PMc averages, as well as the integrated APS and SMPS concentrations shown in the weekly averages in Figure 24. The relative hour of day averages for the particle sizing instruments closely follow the pattern of the two $\mathrm{PM}_{2.5}$ TEOM units, which is distinct from the $\mathrm{PM}_{10}$ and PMc patterns. The hour of day averages in Figure 29B follow a general time trend with features common to most pollutants and particle size fractions. These include two periods of stable average concentrations: a midday period between 11 AM to 5 PM, and an overnight period between $10 \mathrm{PM}$ and 4 AM. The day/night ratio of these two plateau concentrations is a measure of the ratios of the formation rates divided by the loss rates for these periods. For pollutants that are relatively stable towards deposition or reaction loss (such as $\mathrm{CO}$ or $\mathrm{PM}_{2.5}$ ) the loss rates should be similar, and the day/night concentration ratios should be proportional to the relative formation rates during the stable periods. After the midday plateau there is a steady rise in concentration which may be associated with the reduction of atmospheric mixing after sundown as well as any increase in mobile emissions during the evening rush hour. The morning peak is represented by a broad peak between 4 and 8 AM with different relative heights for the fine and coarse size fractions. The morning peak may be associated in part with increased morning emissions during the period of low atmospheric mixing, and is terminated by the increased mixing due to the breakup of the stable atmospheric layers associated with overnight thermal inversions. This breakup can also be associated with fumigation effects where pollutants are returned to ground level after being trapped aloft in these stable levels overnight. While the mixing/dilution loss term will be the same for all pollutants, such fumigation effects will be sensitive to the height of any trapped emissions plumes, which in turn will depend on the location and exit temperature of the emissions sources. Other than this complication, the relative height of the morning peak for different pollutants should be an indicator of the relative emissions of each pollutant in the overnight and early morning period. Applying these concepts to Figure 29B, we note the higher morning peak for PMc compared to $\mathrm{PM}_{2.5}$ (relative to the overall average concentrations of each) and hypothesize that a greater portion of PMc emissions may occur during the overnight/early morning time period. Similarly, from its lower day/night ratio, we hypothesize a lower production rate for PMc relative to its loss rate during the daytime steady state periods than is the case for $\mathrm{PM}_{2.5}$.

Figure 30, analogous to Figure 25, contains data from the Radiance 904 Nephelometer and R\&P 8400S sulfate monitor averaged in relative hour of day units over the 8688 common hours of operation of these two instruments and the TEOM. The nephelometer Bsc averages follow the same general time pattern as the $\mathrm{PM}_{2.5}$ averages, but are less variable on an hourly basis, as shown by lower day/night ratio and lower morning peak than the $\mathrm{PM}_{2.5}$ reference curve. In contrast, neither the $\mathrm{PM}_{2.5}$ sulfate nor gaseous $\mathrm{SO}_{2}$ measured by the $8400 \mathrm{~S}$ follows the general trend. The sulfate averages show only a weak morning peak signature, possibly shifted to an hour later in the morning. The average concentration reaches a broad maximum in the early afternoon, and is lower overall in the night than the day. These features are reasonable if the sulfate is predominately a secondary pollutant that largely arises outside the local airshed. A remotely generated pollutant would not show the overnight accumulation pattern of locally generated pollutants. Further, an afternoon maximum is consistent with enhanced photochemical sulfate. Ambient $\mathrm{SO}_{2}$ concentrations as measured by the $8400 \mathrm{~S}$ monitor also are lower in the night than during the daylight hours. In the place of a morning peak at 6 to $7 \mathrm{AM}$, the $\mathrm{SO}_{2}$ 
average rises to a maximum after $8 \mathrm{AM}$, then falls gradually over the course of the day to a minimum around $6 \mathrm{PM}$ and remains low overnight. The daytime pattern in the figure is actually not seen in practice; typical daily plots of $\mathrm{SO}_{2}$ concentrations show one or more peaks per day with widths of 1 to 4 hours and falling at irregular times. The time of day average in Figure 30 is the composite of this collection of highly variable daily time sequences. The average $\mathrm{SO}_{2}$ data from the ARA gas monitor shows a similar hour of day pattern during the day, but rises after 6 PM to an intermediate plateau from midnight through about 6 AM. The reason for this difference is not clear; it is possible that the overnight $\mathrm{SO}_{2}$ concentrations are in fact different at the $10 \mathrm{~m}$ ARA inlet height and the $3 \mathrm{~m}$ inlet of the $8400 \mathrm{~S}$.

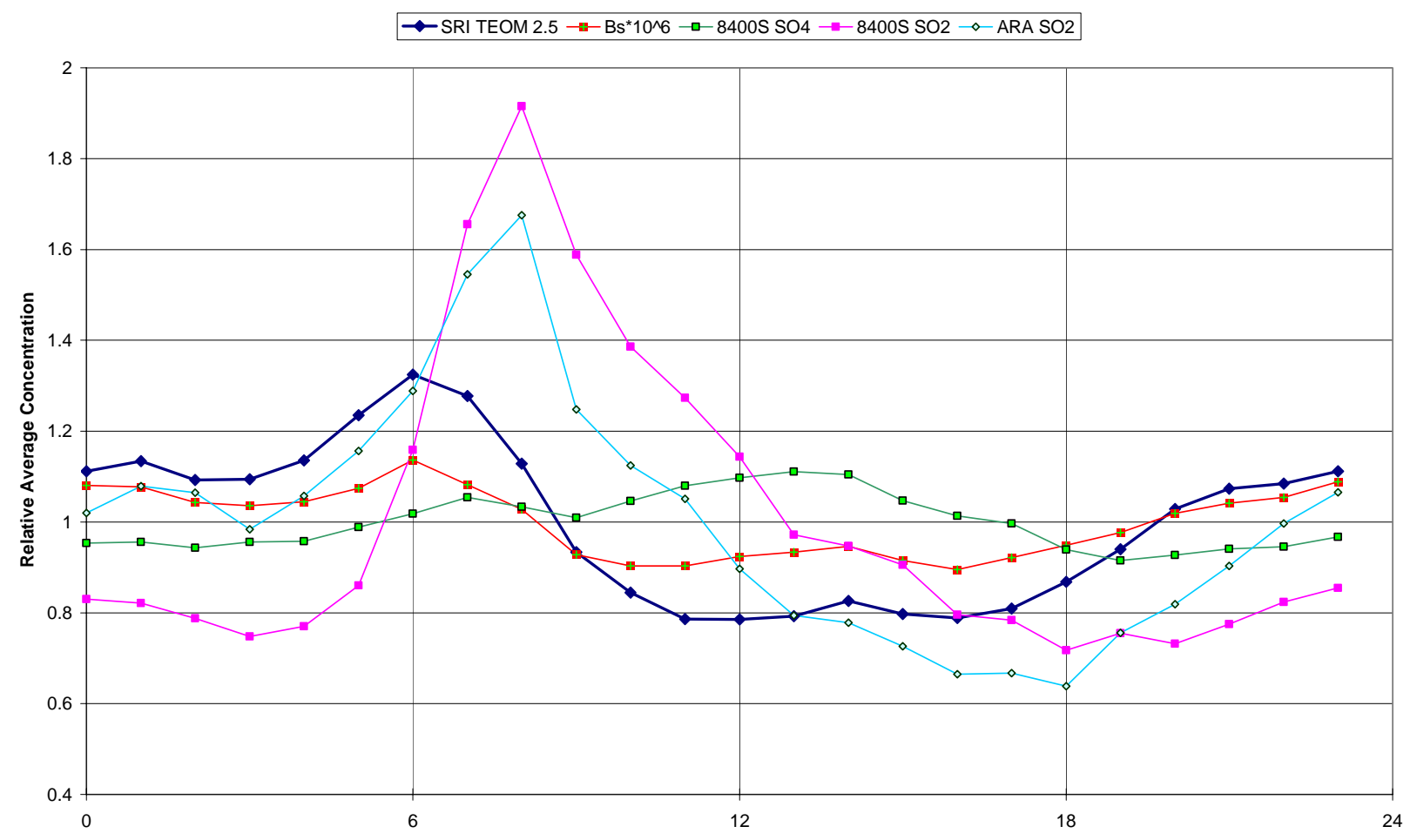

Figure 30. Hour of day relative average data from nephelometer and $8400 \mathrm{~S}$ monitor.

Figure 31, like Figure 26, contains data from the TSI 3496 SMPS averaged over the 7407 common hours of operation of this instrument and the SRI TEOM. Hour of Day averages from several indicator size fractions, as well as the TEOM for reference, are included in the plot. While all size fractions follow the typical pattern, there are significant differences in the behavior of the size groupings. The total integrated mass-weighted size distribution and the portion having diameter larger than $0.5 \mu \mathrm{m}$ (SMPS 0.5-1.0) both track the TEOM PM 2.5 closely over the day; this is not surprising since these SMPS size ranges represent a large portion of the $\mathrm{PM}_{2.5}$ concentration and are inherently highly correlated at all times. The smallest particles measurable in our operating protocol are in the first (21.3 nm median diameter) SMPS channel. These particles have a large morning peak ( 1.56 times the overall average of 55 per $\mathrm{cm}^{3}$ channel count) but instead of a rapid morning dropoff to minimum daily levels after $10 \mathrm{AM}$, this size range passes through an inflection around $11 \mathrm{AM}$ and slight maximum at noon before falling to a daily minimum after 4 PM. In the evening these particles rise to a plateau level, and after around 9PM begin to fall through the night to a minimum after 2 AM before rising to the next morning peak. 
The next two larger inclusive size groupings (N30 - number between 20-30 nm - and N20-50) show similar patterns, except that the daytime minimum averages are progressively lower, and the daytime secondary peak falls at progressively later times, and the overnight minimum averages are progressively higher. Thus the day/night ratio decreases as the ultrafine size band is extended, while the relative height of the morning peak remains at 1.56 . The next larger band of particles (N50-100), continues this trend, with morning peak of 1.59, the lowest day/night ratio, and no clear afternoon peak or early morning falloff. The mass-weighted $\mathrm{PM}_{100}$ essentially consists of the same particle set and follows the same time course. The total integrated particle number tracks the latter two bands, but with slightly lower morning peak and higher day/night ratio. The behavior of the average size bands in the figure is an indication of fairly rapid evolution of the ultrafine particle size distribution during the day with much smaller changes in the accumulation mode size range containing most of the particle mass.

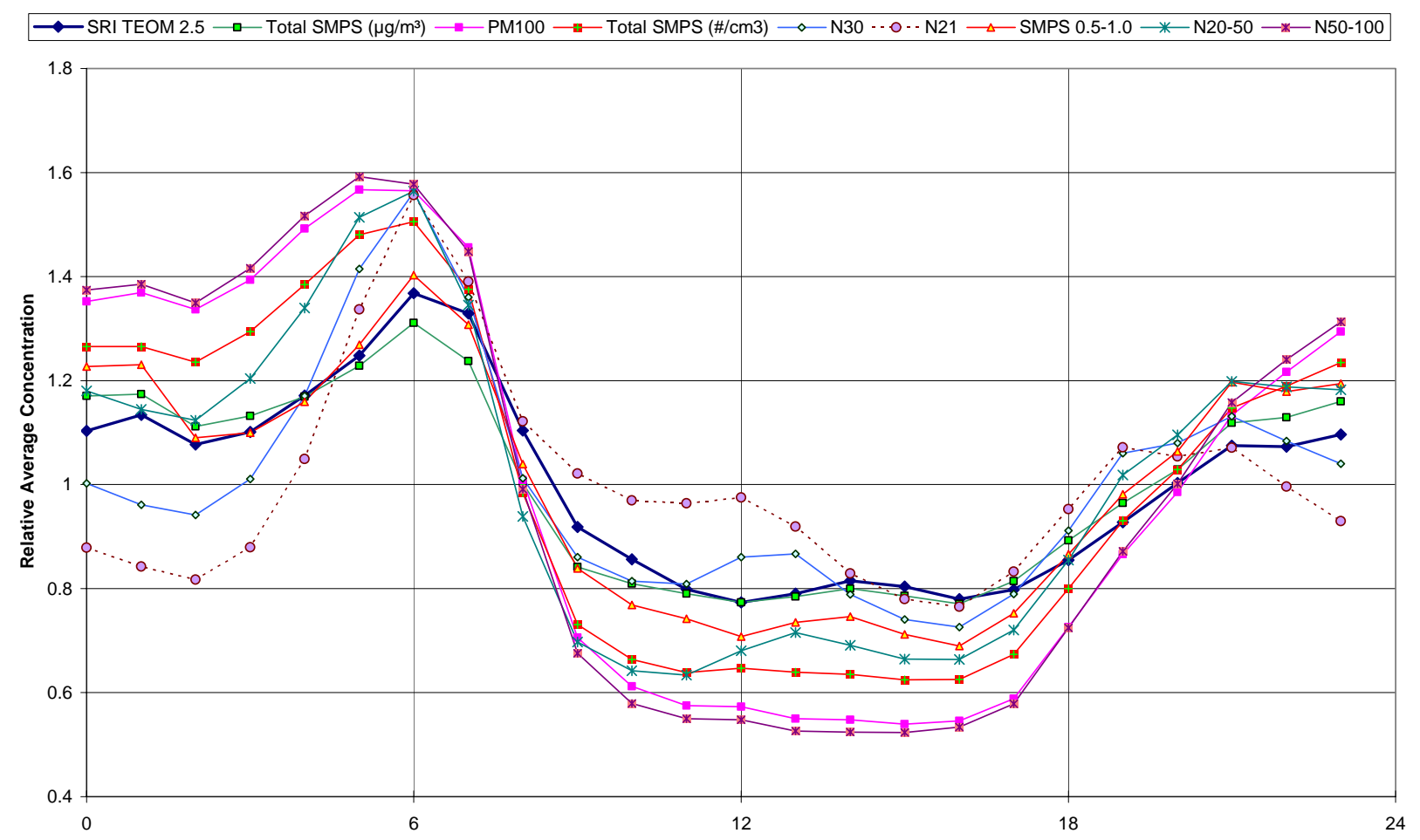

Figure 31. Hour of day relative average submicron size fractions from SMPS.

Like Figure 27, Figure 32 is an analogous plot using size fractions from the APS 3321 aerodynamic particle sizer, averaged over 2452 hours during March - August, 2002. As in Figure 27, the SRI PM 2.5 TEOM and the calculated PMc averages from the AIRS TEOMs are presented for comparison. Again, the size fractions spanning $0.5-1.0 \mu \mathrm{m}, 1.0-2.5 \mu \mathrm{m}$, and 2.5 $-10 \mu \mathrm{m}$ progressively follow a trend toward increasing morning peak concentration and decreasing day/night ratio as the mean diameter of the size band is increased. The larger size bands lie between the $\mathrm{PM}_{2.5}$ and $\mathrm{PMc}$ reference curves 


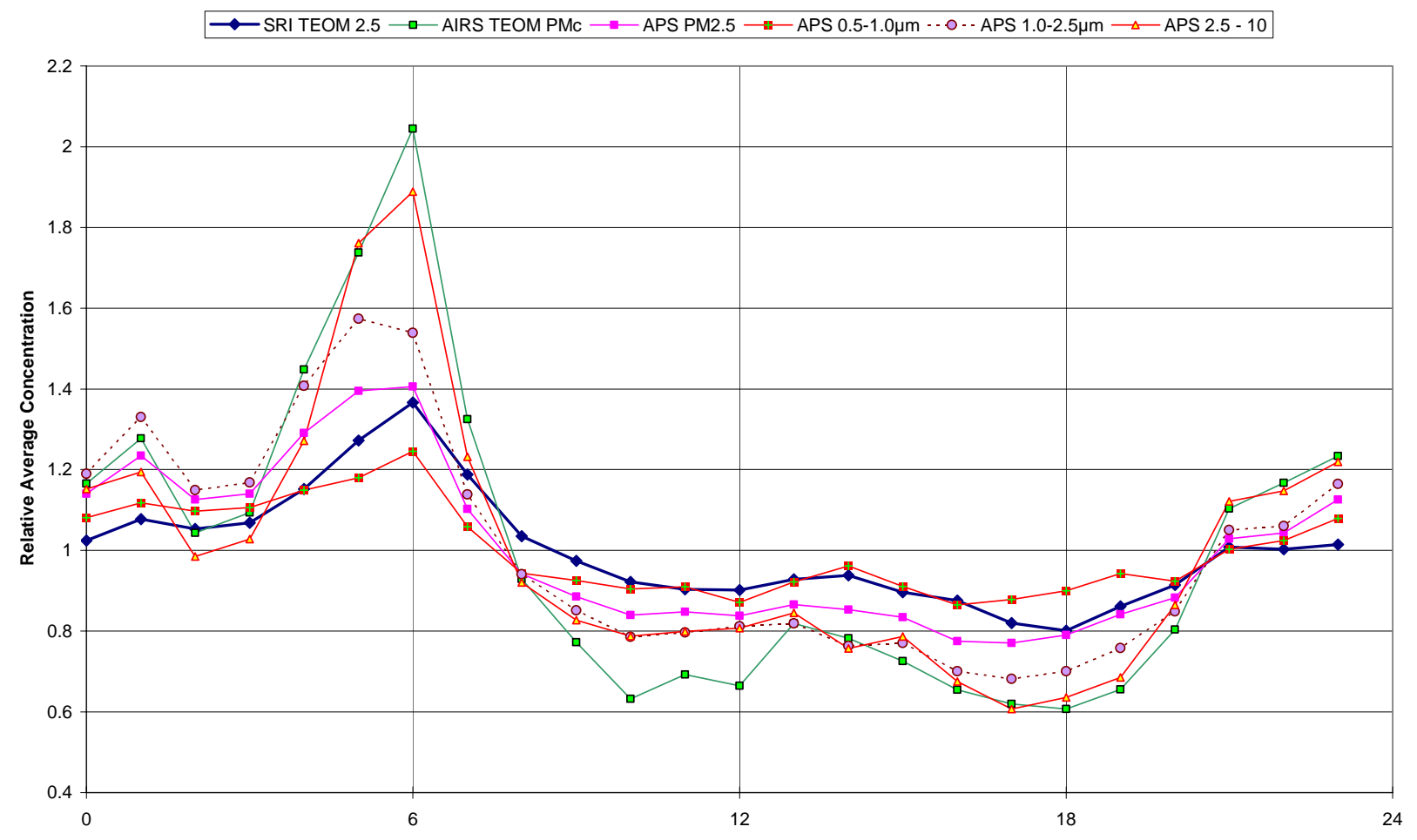

Figure 32. Hour of day relative average APS 3321 size fractions (after March, 2002).

Finally, Figure 33, like Figure 28, contains hourly average published ARA data from the onsite continuous gas monitors averaged over 11448 common hours of operation with the SRI TEOM, and a variable numbers of matching hours for the other instruments. As discussed in the weekly behavior of the gases, atypical hourly patterns are observed for $\mathrm{SO}_{2}$, discussed above, and for $\mathrm{O}_{3}$. The daily ozone pattern tracks the solar radiation pattern of the daylight hours, as should be expected for this secondary pollutant which is the product of photochemical atmospheric reactions. The daily $\mathrm{CO}$ pattern again most closely follows the prototype $\mathrm{PM}_{2.5}$ trend, with similar morning peak height but smaller day/night ratio. Average NO again has a time of day pattern most comparable to PMc, though even more exaggerated in this case, with the highest relative morning peak concentration (2.33 times average) and lowest day/night ratio of any of the pollutants included here. Again, its atmospheric reaction product, NOy, follows a similar but less exaggerated pattern.

Examination of the data presented in this section indicates that the pollutants with the greatest variability by day of week (e.g., NO and PMc) also show the greatest variability within the typical hour of day trend. Figures 34-38 examine the interaction of the diurnal and weekly time scales for these and other key pollutants. Figure 34 is an overlay of hour of day averages of the ARA NO monitor data for each day of the week, and the subsequent plots depict analogous data for $\mathrm{PM}_{10}, \mathrm{PMc} \mathrm{PM}_{2.5}$, particle sulfate, and $\mathrm{SO}_{2}$, each of which has distinctive behavior as discussed above in this section. Averages for Saturday and Sunday are labeled and plotted with bold lines in the figures. Individual weekdays are not generally distinguished. 


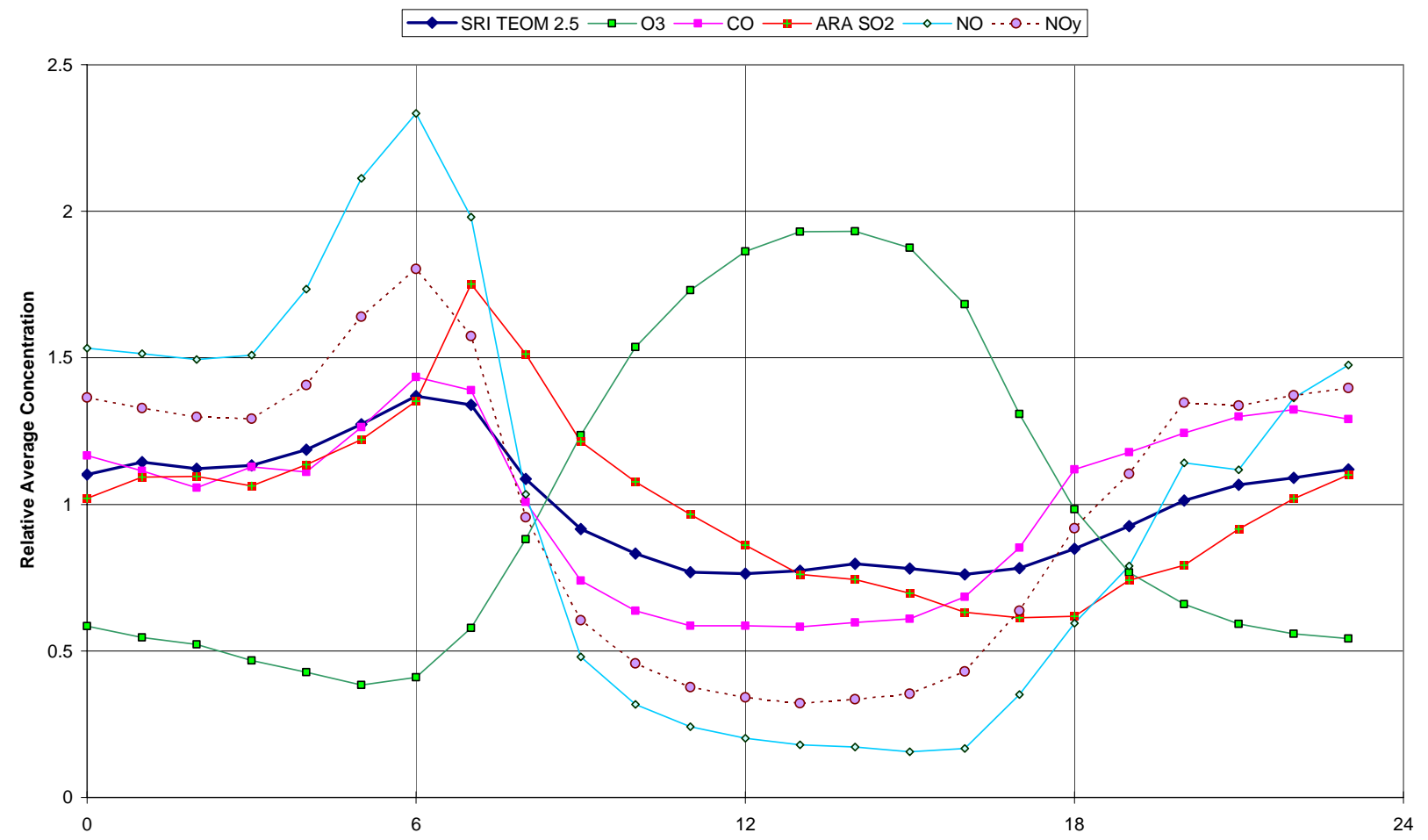

Figure 33. Hour of day relative average concentrations from ARA gas monitor data.

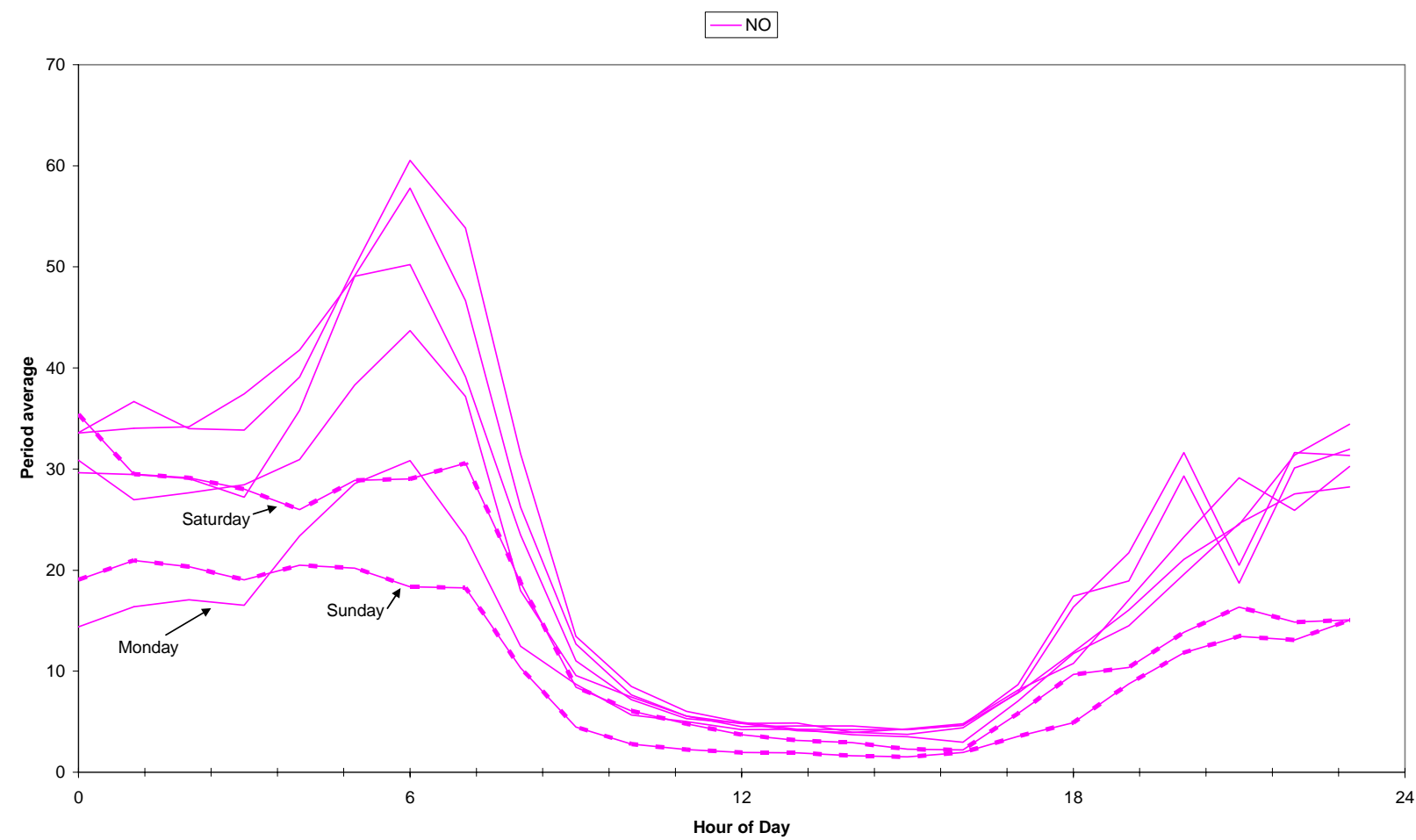

Figure 34. Hour of day ARA NO concentration average overlay for each day of the week. 
The weekday hour-of-day patterns in Figure 34 all follow the general trend seen in the weekaveraged composite NO plot in Figure 33, though there is day to day variation in the scaling of the morning concentration curves (midnight to noon) and hour to hour scatter in the evening averages on two of the days. Beginning on Saturday morning is a trend toward lower concentrations that progresses through the weekend. The Saturday morning peak is significantly lower than weekday values, barely rising above the downward slope of the overnight baseline, and the concentration falls progressively lower relative to the corresponding weekday value for the remainder of the weekend through the early morning period on Monday (also labeled on this figure), which is lower then the corresponding period of any day of the week. On Sunday the morning peak is essentially absent, and the reduced baseline on Monday causes the Monday morning peak to be $20 \mathrm{ppb}$ lower than the typical weekday value.

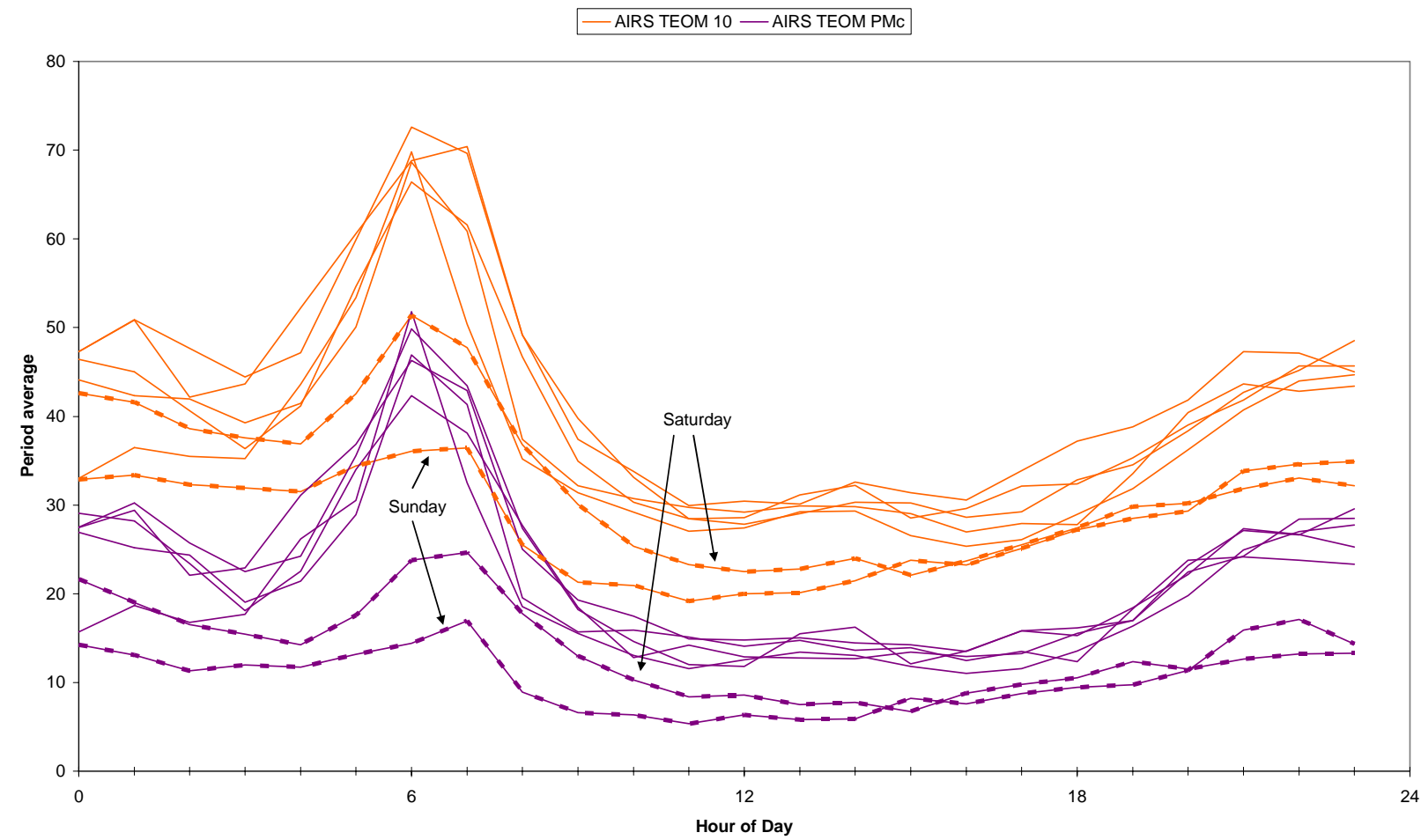

Figure 35. Hour of day $\mathbf{P M}_{10}$ and PMc concentration average overlay by day of the week.

The time behavior of NO in the Figure 34 is typical of that seen for several other pollutants. Analogous plots for $\mathrm{PM}_{10}$ and for calculated PMc (both presented in Figure 35) show similar features, including the height of the morning peak, which is again dramatically reduced on Saturday and nearly eliminated on Sunday. The second general effect is the progressive drop in concentration (relative to the average weekday value) over all times during the weekend, which is not reversed until early Monday morning. The association of the overnight concentrations with the concentrations of the previous day is seen most clearly on Monday and Saturday, the days for which the mean concentration levels change most from those of the previous day. This effect is the rationale for using the five hour time lag in the assignment of days for the day of week calculations earlier in this section. 


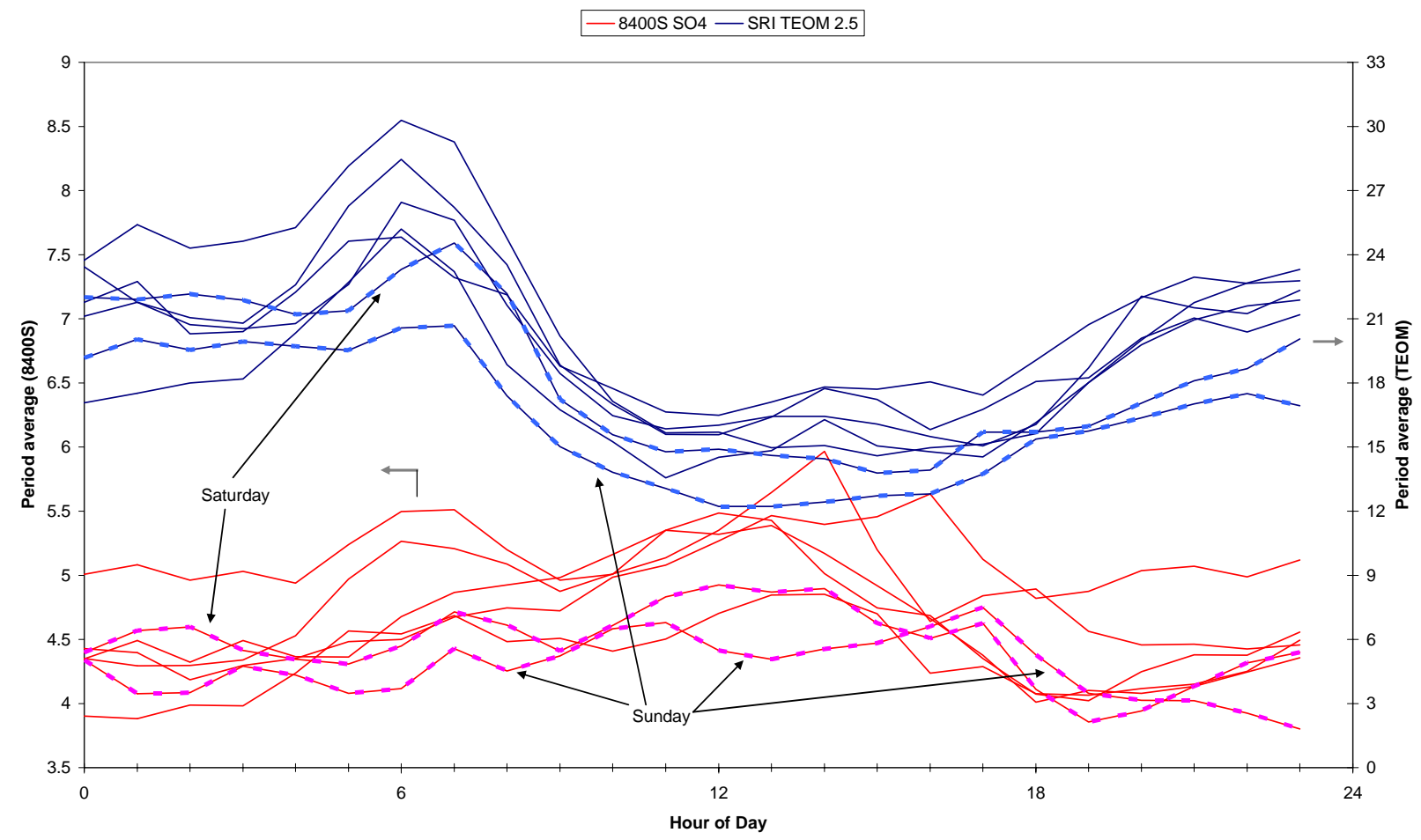

Figure 36. Hour of day $\mathbf{P M}_{2.5}$ and sulfate average overlay by day of the week.

Figure 36 presents a comparable plot for $\mathrm{PM}_{2.5}$ from the SRI TEOM and particle sulfate as measured by the $8400 \mathrm{~S}$ sulfate monitor. The average plots from the two instruments are shown on offset scales for visibility. The $\mathrm{PM}_{2.5}$ TEOM averages follow the general trends seen in the $\mathrm{NO}$ and PMc data, but with less day-to-day variation. The progressive reduction in hourly concentration over the weekend relative to corresponding weekday hours is seen on both days. While the Sunday morning peak is again essentially eliminated, the Saturday peak is within the range of the weekday values. The Saturday morning peak appears to occur somewhat later than on any of the weekdays, though at the one hour time resolution of the figure this is not clearly the case. Figures 34 and 35 show the same ambiguous suggestion that the Saturday morning peak may fall less than an hour later for NO and PMc respectively; however, none of the plots are conclusive. Such a weekend delay in the morning peak is quite plausible given the lower level of early rush hour mobile source emissions on Saturday. The sulfate data in the figure provide some insight into the atypical composite hour of day pattern seen in Figure 30. In Figure 36 the day to day scatter in the hour of day curves exceeds the relatively small variations in the individual curves. Within these variations, a credible pattern of lower Saturday and Sunday concentrations does persist, as well as a tendency toward slightly higher afternoon concentrations on all days other than Sunday. The hourly structure in these curves is otherwise inconsistent: while most days appear to show relative maxima in the morning and in the afternoon, the height and peak time of these features shift from day to day and do not seem to represent a repeating time pattern.

Figure 37 presents a comparable plot of the gaseous $\mathrm{SO}_{2}$ as measured by the $8400 \mathrm{~S}$ sulfate monitor along with the comparison $\mathrm{SO}_{2}$ data from the ARA monitor. As in the previous figure, the average plots from the two instruments are shown on offset scales for visibility. The irregularity in the daily plots for $\mathrm{SO}_{2}$ is even more pronounced than for the sulfate data. As 
mentioned in the discussion of Figure 30, the hour of day $\mathrm{SO}_{2}$ average represents a composite average of a time series with irregularly spaced peaks. The difference in the daily plots in Figure 37 indicates that the time pattern of these peaks is also irregular on a day of the week basis, but the overall envelope of daytime concentrations is followed on all days of the week. The Saturday and Sunday time sequences lay on the lower edge of the envelope of the weekday plots. The higher Tuesday concentrations are again seen by both instruments, and consist of higher average concentrations during almost all hours of the day after 8 AM.

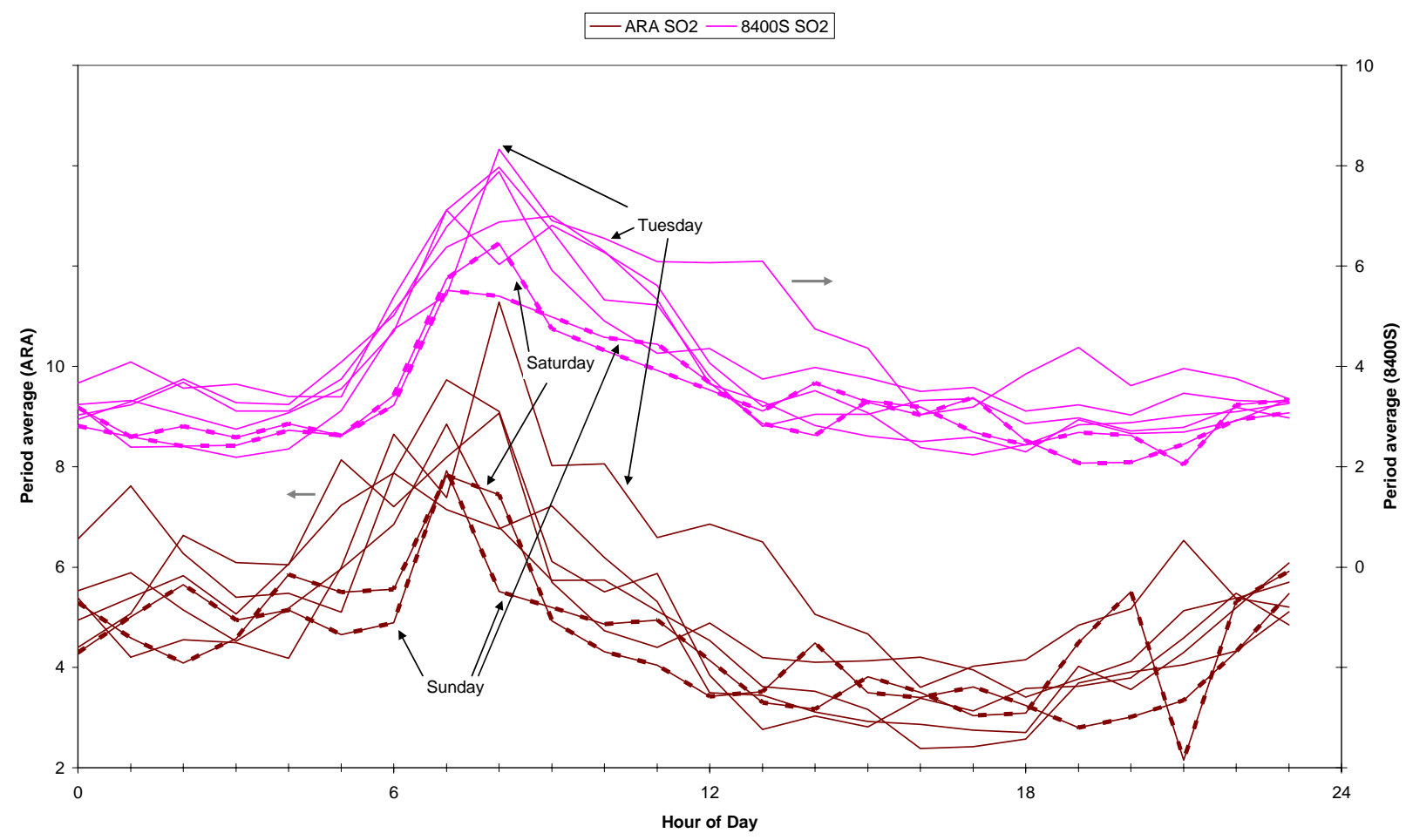

Figure 37. Hour of day $\mathrm{SO}_{2}$ average overlay for ARA and 8400S monitor data.

\section{Comparative Measurements of Particulate Properties and Composition}

$\underline{\mathrm{PM}_{2.5}}$ mass concentration measurements

During the period after initial installation of the $\mathrm{PM}_{2.5}$ TEOM and the end of the project period on 9/30/2001, 469 days of complete $\mathrm{PM}_{2.5}$ daily averages (calculated using $>21$ valid hourly averages of data) were obtained. Of these, 452 correspond to valid daily $\mathrm{PM}_{2.5}$ concentrations reported by JCHD from their $\mathrm{PM}_{2.5}$ FRM monitor at the site. Also reported on AIRS were 429 complete days of data from their $\mathrm{PM}_{2.5}$ TEOM after it began operation on 7/14/2001. Of these, 362 correspond to complete days of our TEOM. In addition, 400 days had over 12 valid hours of common data from the two $\mathrm{PM}_{2.5}$ TEOM monitors suitable for instrument comparison. Using this set of common hourly and daily average data, $\mathrm{PM}_{2.5}$ concentration comparisons for these three instruments can be made over six calendar quarters. The results of these comparisons are presented in Figures 38 - 43. 


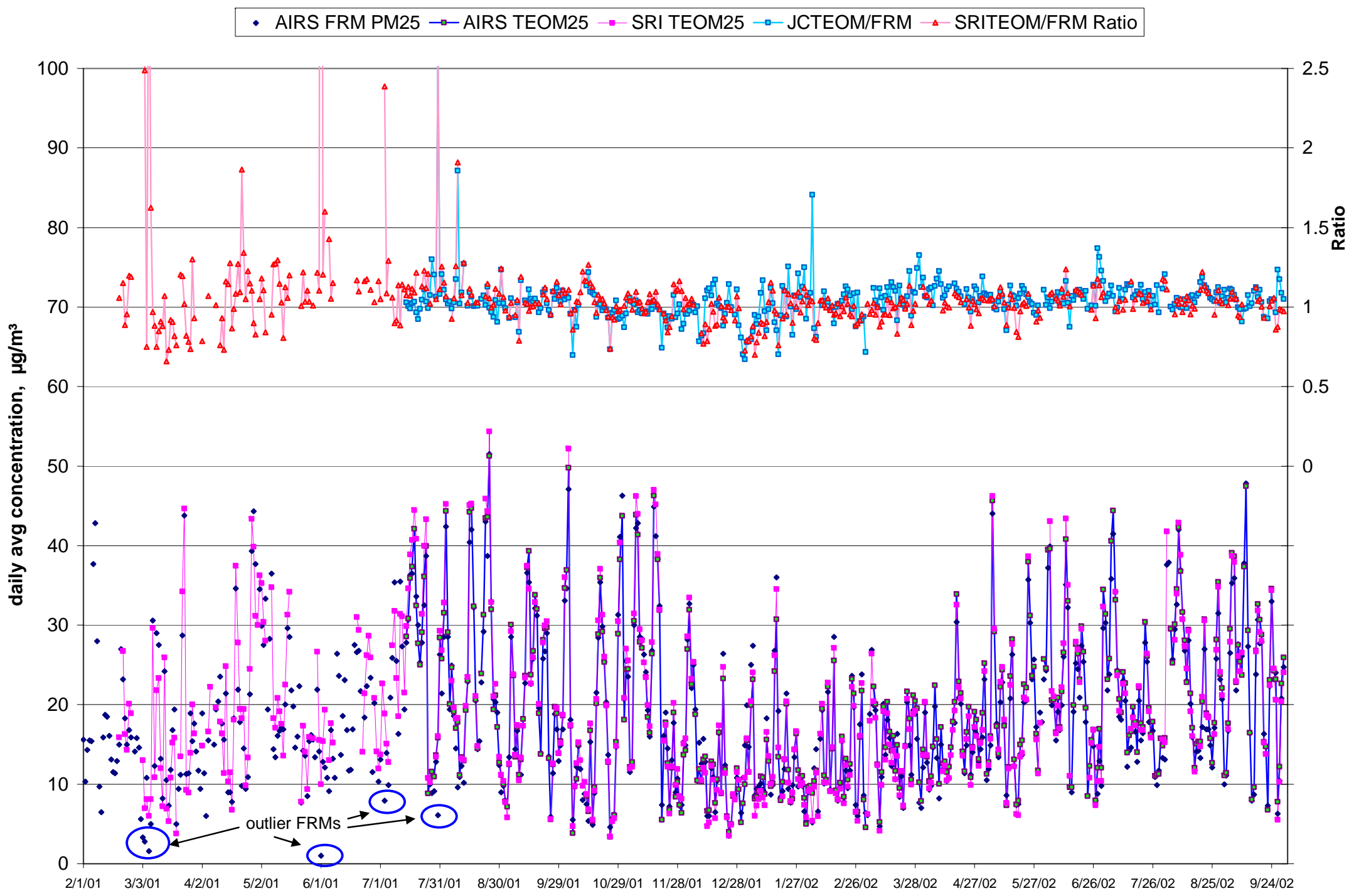

Figure 38. Daily average PM PM. $_{2.5}$ concentration as measured by SRI and JCHD TEOMs and JCHD FRM. Ratios of TEOM averages to FRM are also shown. 


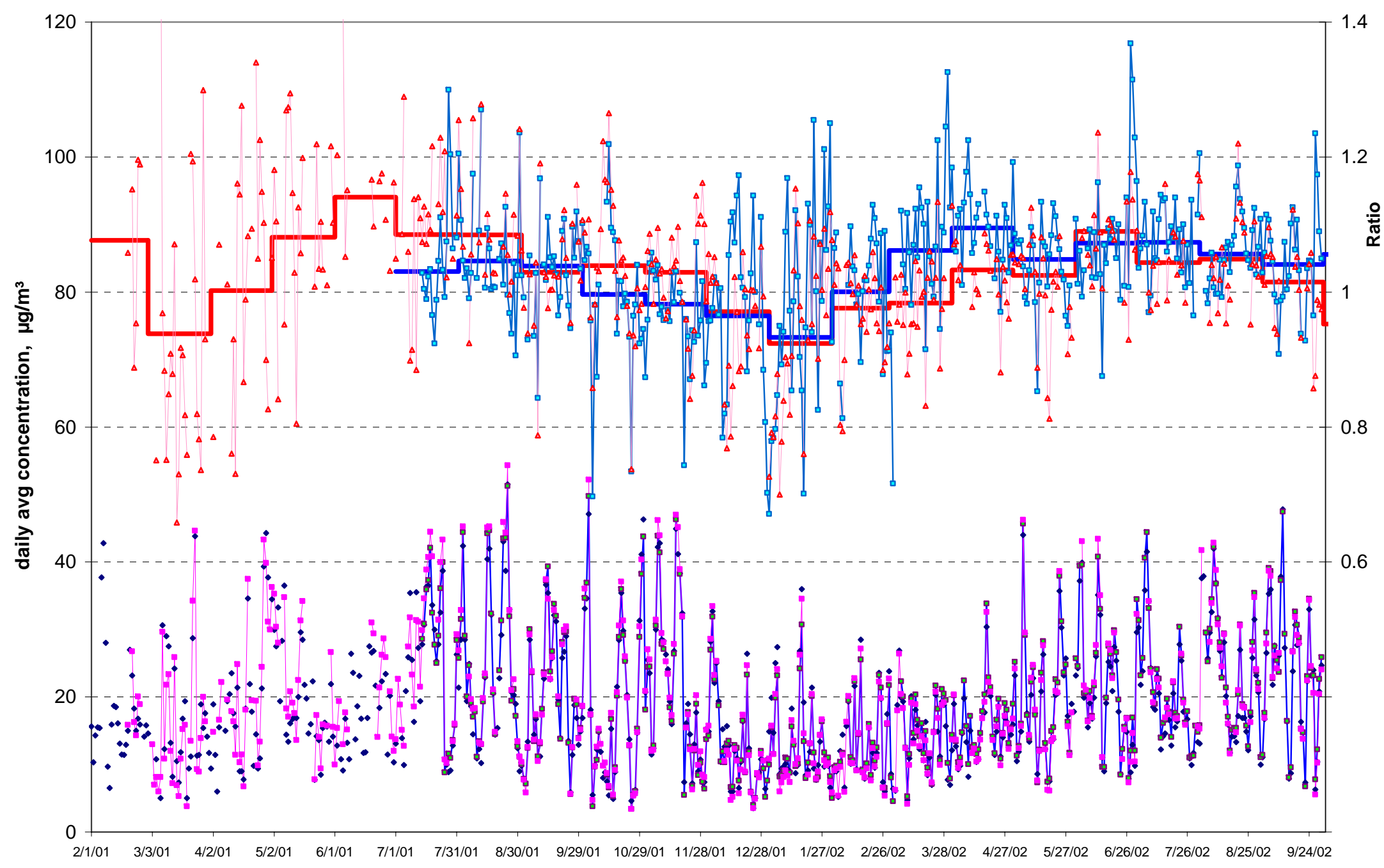

Figure 39. Daily average $\mathbf{P M}_{2.5}$ concentrations from Figure 38 with outliers deleted. Ratios of corresponding TEOM and FRM daily and monthly averages are also shown on expanded scale. 


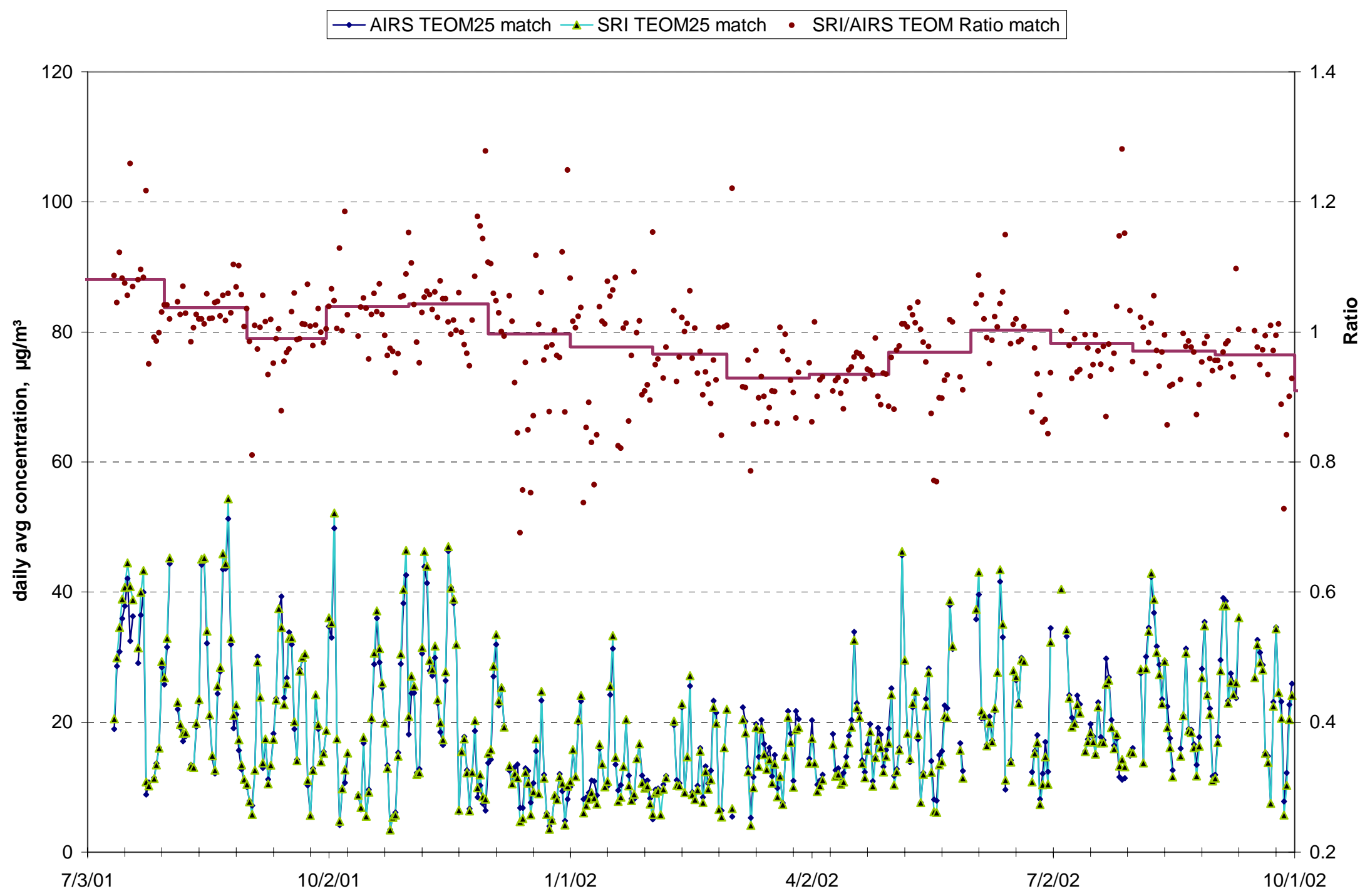

Figure 40. Daily average PM $\mathbf{P M}_{2.5}$ concentrations as measured by SRI and JCHD TEOMs. Ratios of daily averages for corresponding hours are also shown on expanded scale. 
Figure 38 presents daily average $\mathrm{PM}_{2.5}$ mass concentrations measured by the FRM and two $\mathrm{PM}_{2.5}$ TEOM monitors over the course of the project time period. On the second axis, the plot also contains the ratio of each daily average TEOM concentration to the corresponding daily FRM concentration. While the majority of the concentration ratio values fluctuate within the range 0.93-1.07, in the early portion of the project the scatter is larger and includes some clear outlier days. Comparison of the individual concentrations with data from other instruments indicates that most of the anomalous ratios can be attributed to low reported values for the FRM measurements. The most striking of these are marked on the figure. Of the set of ratios that lie outside the range $0.7-1.4$, nine FRM outliers were eliminated, two SRI TEOM days, and one JCHD TEOM day. On a few days it was not possible to determine the instrument responsible for the suspect ratio. Several less extreme deviations of the ratios from unity fall in the period after the JCHD TEOM was operational. On many of these days as well the two TEOMs agree with one another more closely than with the FRM.

Figure 39 presents the same daily averages and ratios without the outlier points on an expanded scale. To reduce the scatter in the ratios, monthly average ratios for each TEOM are plotted in the figure as bold histograms overlaying the daily average ratios. These are calculated over corresponding complete days as follows. For each month and device, daily averages are included only if valid corresponding FRM days (excluding missing or outlier days) are present. Monthly averages are calculated for each device using these coincident days, and the plotted monthly ratio for each TEOM is the quotient of these coincident monthly averages for that device and the FRM. The monthly ratios of both TEOMS follow a common trend that is roughly seasonal. This trend continues over four years in the published AIRS data for the JCHD devices, with lower ratios around December - January each year, and higher ratios in the spring and summer months. This is similar to the cycle of relative $\mathrm{PM}_{2.5}$ concentrations, though in each of the four years the minimum ratio occurs a month before the minimum concentration. Seasonal variations in the ratio of FRM and TEOM measurements have been reported in several studies, and are generally attributed to temperature-related differences in measurement of semivolatile materials and of particle-bound water.

Figure 40 contains a similar comparison plot for the two TEOMs. On the main axis of the figure are daily average values for the 400 days with over 12 simultaneous measurement hours of each TEOM. For comparison purposes only the corresponding hours are included in each daily average. On the second axis the SRI/JCHD TEOM ratios are plotted for both daily and monthly corresponding averages. The TEOM/TEOM ratios show somewhat less scatter than either TEOM/FRM pairing, with relative standard deviation (RSD) of $8.4 \%$ over the period of common TEOM operation compared to 9.2 and 9.5\%, respectively, for the SRI and JCHD TEOMS ratios to the FRM during this period. (During the period before July 13, 2001, the RSD of the SRI TEOM/FRM ratio was $17 \%$ even after elimination of the eleven outlier days in this period). The monthly ratios in the figure show longer term trends, but the pattern is less clearly seasonal in nature than in the TEOM/FRM cases. The SRI TEOM on average reads higher than the JCHD device during 2001, but lower throughout 2002. The ratio of the TEOMs seems to decline steadily to a monthly mean of 0.93 in March, 2002, then vary between that level and 1.0 for the remainder of the project period.

Figure 41 contains a scatter plot comparison of daily average $\mathrm{PM}_{2.5}$ concentrations for the two TEOMs relative to the FRM during the project period. Regression equations for each device are shown on the figure. The outlier days described above were eliminated prior to the plot and 
regressions. The scatter bands for both devices overlap closely, and the regression fits for both fall well within the scatter bands. The center of gravity of both sets of points lie a few percent above a 1:1 relationship, with the SRI TEOM marginally higher for higher concentration days. The fits are of comparable quality. The SRI TEOM regression as shown has lower $\mathrm{r}^{2}$, but this difference is entirely due to the greater scatter in the days before the JCHD TEOM became operational on 7/13/2001. If these earlier days are eliminated from the SRI TEOM data set, the fit $\mathrm{r}^{2}$ improves to 0.9781 .

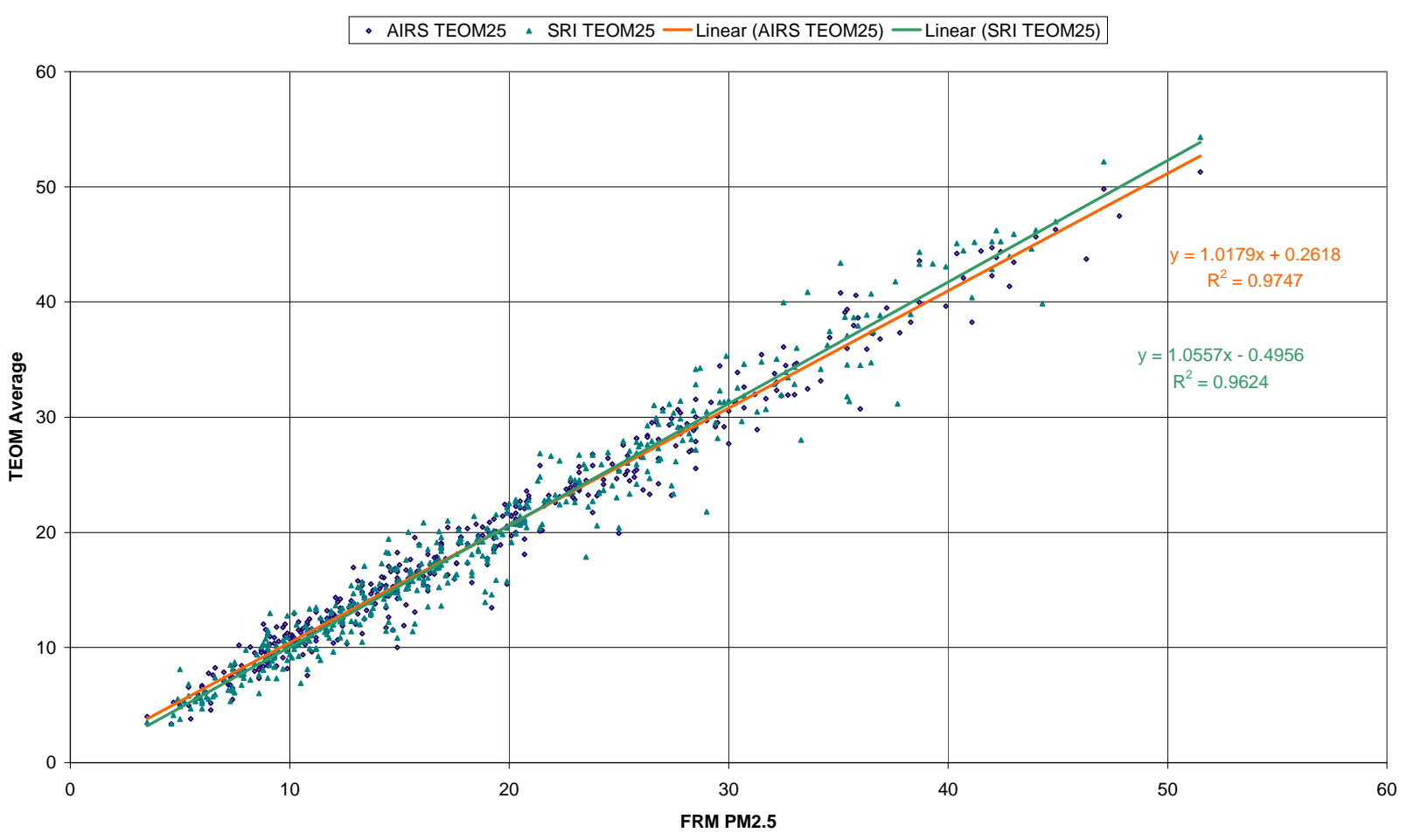

Figure 41. Comparison of daily average PM$_{2.5}$ TEOM concentrations with JCHD FRM over the project period.

Figure 42 contains a similar scatter plot comparison of daily average $\mathrm{PM}_{2.5}$ concentrations for the SRI TEOM relative to the JCHD TEOM. Two data sets are shown on the plot. The set labeled "SRI TEOM25 match" contains the data set from Figure 40: that is, averages of only corresponding hours from the 400 days having over 12 such common hours for both TEOMs. The other set contains daily average pairs from the 362 simultaneous "complete" days for both devices. Regression equations for each data set are again shown on the figure. The two fits are essentially identical; both suggest an offset of roughly $1 \mu \mathrm{g} / \mathrm{m}^{3}$ and $5 \%$ slope difference for the TEOMs. Figure 43 is a direct plot of the 9457 corresponding hourly averages used to construct the "SRI TEOM25 match" data set in Figure 42. There are several clear outliers in the hourly average set, generally associated with an upset or filter change on one of the devices. A reduced set is also plotted in the figure in which 35 points were eliminated, including hourly averages less than zero and those differing by more than $13 \mu \mathrm{g} / \mathrm{m}^{3}$. Regression fits for the original and culled sets are shown on the figure. The fit equation for the trimmed data set essentially overlays that for the daily averages. 


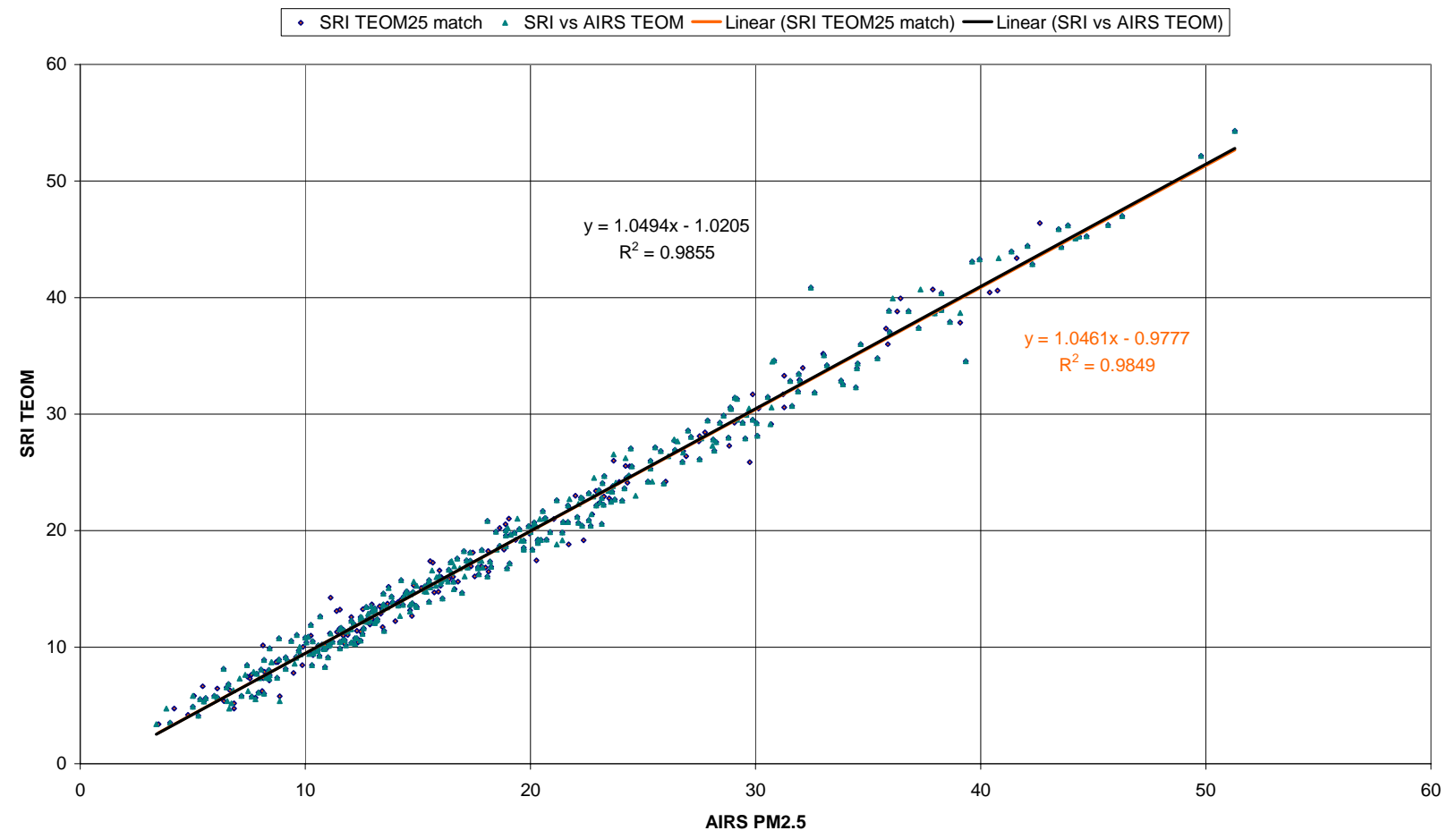

Figure 42. Comparison of daily average $\mathbf{P M}_{2.5}$ concentrations for corresponding hours measured by SRI and JCHD TEOMs over the project period.

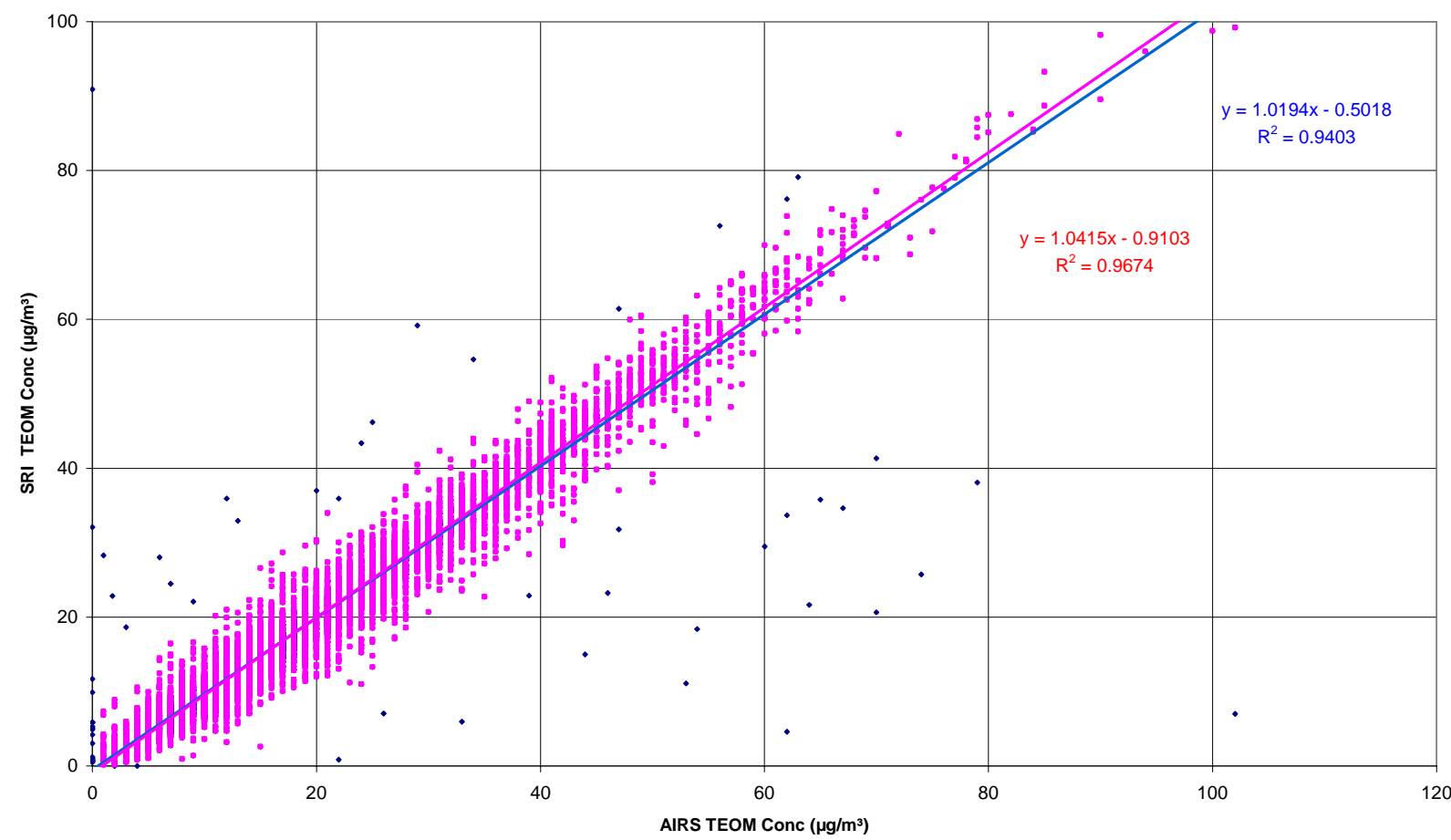

Figure 43. Comparison of corresponding hourly average $\mathbf{P M}_{2.5}$ concentrations measured by SRI and JCHD TEOMs. Outliers shown were eliminated for fit. 


\section{Submicron PM fractions and size distribution: SMPS data}

The particle sizing instruments add a valuable dimension to the suite of measurements at the site, since the measured $\mathrm{PM}_{2.5}$ is a mixture of particle sizes. Particles from specific sources have characteristic size distributions that often may differ enough to identify or estimate the contribution of the source to the ambient $\mathrm{PM}_{2.5}$ mixture at favorable times. The high time resolution available with the sizing instruments makes such assignments possible over the course of a study day.

Although neither of the two sizing instruments used is optimized for precise measurement of overall particle mass concentration, it is instructive to compare the integrated size spectra with the contemporary measurements of $\mathrm{PM}_{2.5}$ from the TEOM or FRM monitors at the site. This is shown in the next several figures for the total size spectrum and for several size fractions measured by the SMPS monitor. Figures 44 and 45 show daily average time series comparisons for fractions weighted on mass and number basis respectively, over the available days of the project period. Figure 44 contains daily average total mass-integrated size spectra for the days where complete ( $>21$ valid hours) SMPS data are available. The mass is calculated using the measured size range from $20 \mathrm{~nm}-1 \mu \mathrm{m}$ physical diameter and an assigned density of 1 . Both the size range and the assigned density will cause the SMPS mass to underestimate $\mathrm{PM}_{2.5}$. For comparison the total mass-integrated size averages time series is overlaid with ambient $\mathrm{PM}_{2.5}$; for completeness, FRM data are used in the figure, though the data from the two TEOMs are comparable. On the second scale are plotted the ratios of the calculated average total and $\mathrm{PM}_{100}$ mass to the ambient $\mathrm{PM}_{2.5}$, in this case measured by the project TEOM. The time series breaks into three periods. The longest period begins when the instrument began operation in the sampling shelter on July 2, 2001 through mid-July of 2002. This period was interrupted for over two months in February - April, 2002 when the instrument was removed from the site and repaired. During this extended time the ratio of total mass to $\mathrm{PM}_{2.5}$ scatters around a value of roughly 0.7 with no clear seasonal trend. Beginning in July the ratio steadily decreases for the remainder of the project period to a value near 0.3 at the end of September. This corresponds to the period of flow calibration failure which resulted in incorrect sample flowrate to the instrument. The initial measurements in April and May, 2001, were made with the temporary sampler configuration in the ARA shelter. During this period the mean average was higher, and the scatter considerably greater. The $\mathrm{PM}_{100}$ ratios on the figure show considerably greater day to day variability at all times, with an apparent span of lower ratios during the summer of 2001.

Figure 45 is a similar plot substituting the number-weighted total size spectrum for the mass weighted averages. The second number basis size fraction is N30 (between 20-30 nm), at the smallest portion of the measured size distribution. Both these size fractions show day to day scatter similar to the mass weighted ultrafine range PM100, and correlate much more closely to it and to one another than to $\mathrm{PM}_{2.5}$. Pairwise comparisons of these size fractions are plotted in Figures 46-47. In order to eliminate the systematic differences seen in the beginning and end of the time series, these correlations were calculated over the one year period from July, 2001 through June, 2002. The figures depict regressions of three mass-weighted and two numberweighted daily average size fractions against daily average TEOM PM 2.5 . The coefficient of determination decreases as the mass-based upper size limit decreases from 1000 to 500 to $100 \mathrm{~nm}$. Similarly, the coefficient of determination decreases with size for the number-weighted size fractions, and is poorer in general for number weighting. 


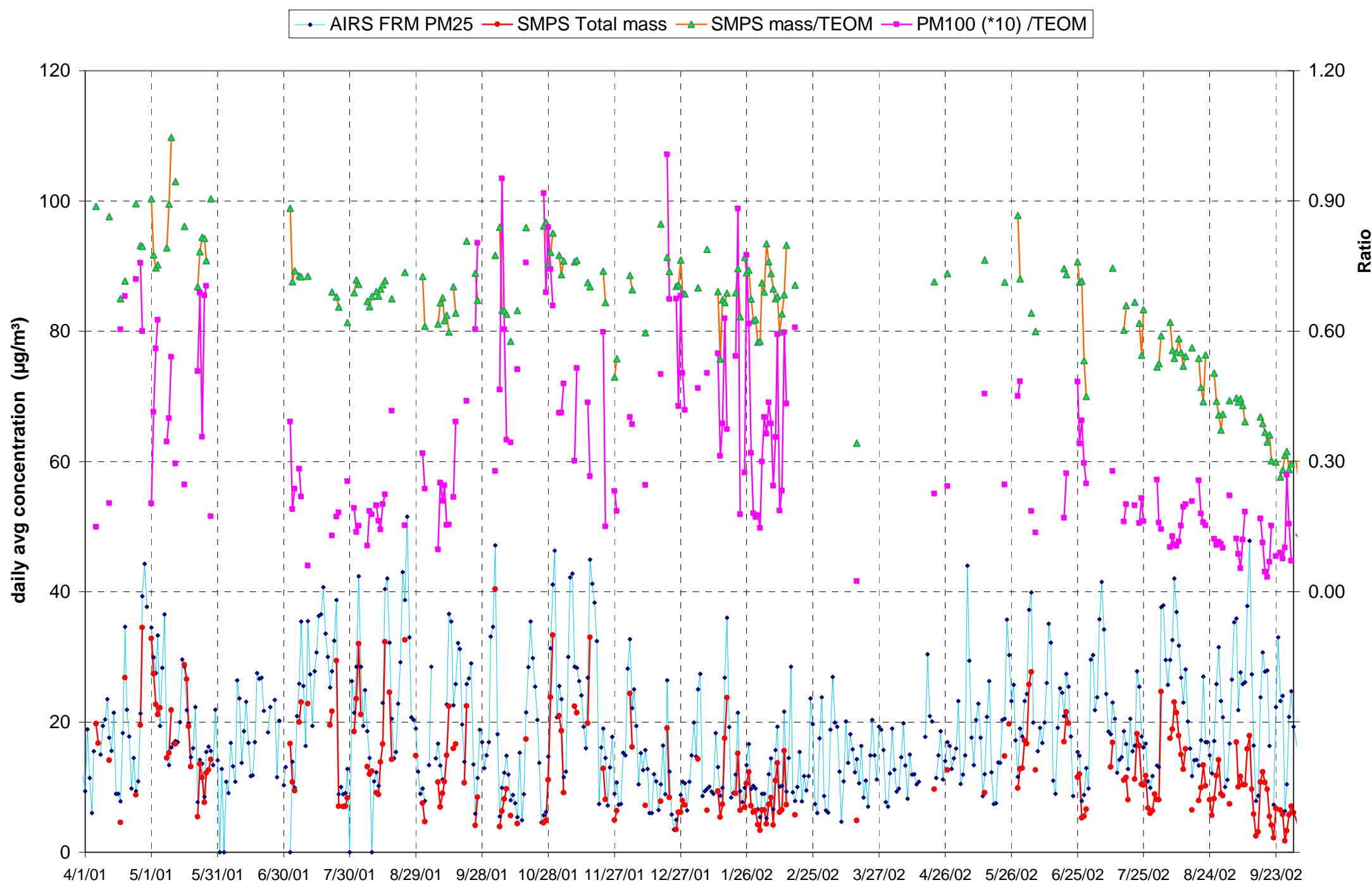

Figure 44. Daily average integrated SMPS mass plotted with PM $_{2.5}$ concentration from JCHD FRM. Ratios of SMPS total and $\mathbf{P M}_{100}$ mass averages to SRI TEOM PM $\mathbf{P M}_{2.5}$ are shown on second scale. 


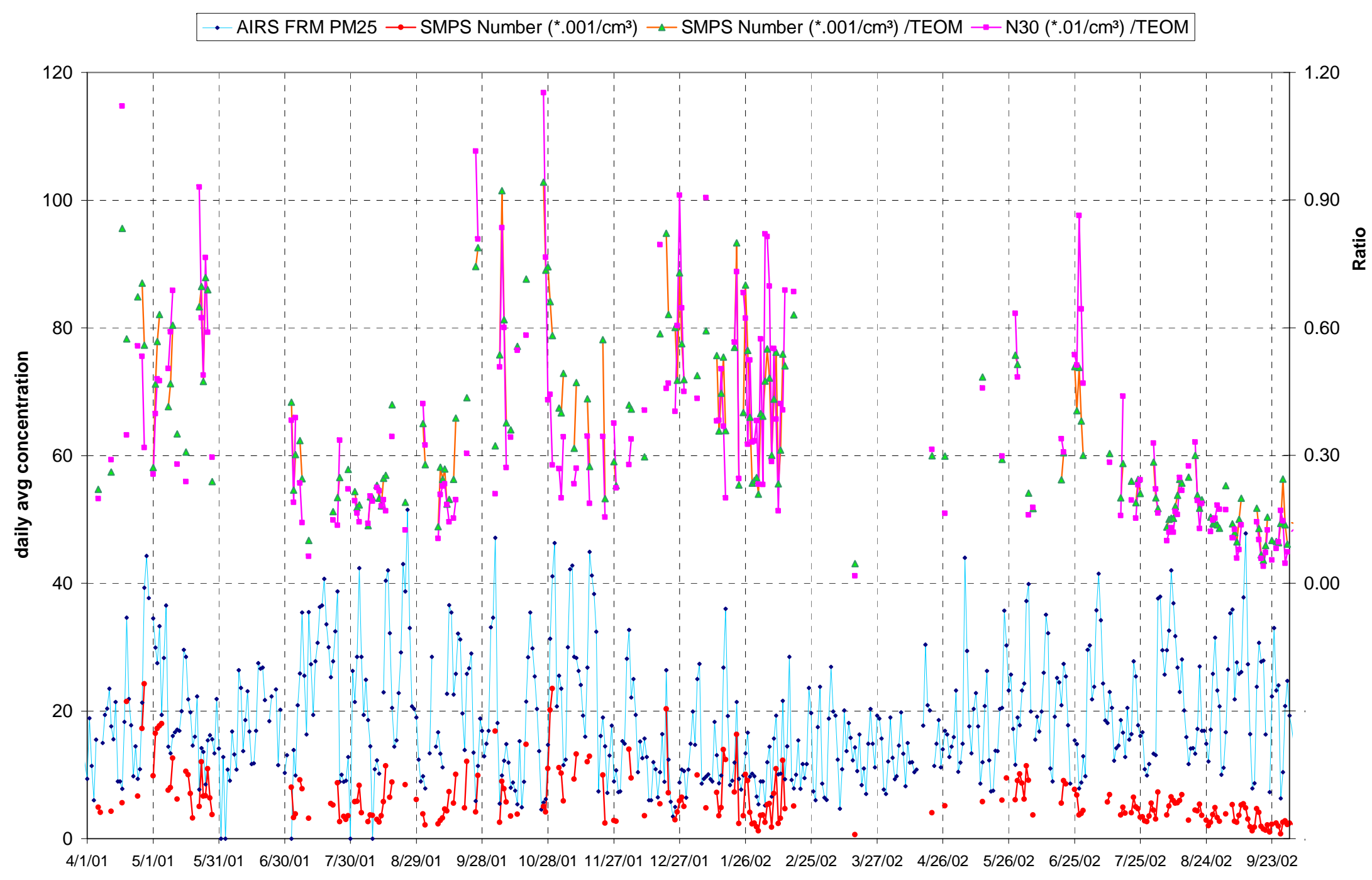

Figure 45. Daily average integrated SMPS number plotted with PM $_{2.5}$ concentration from JCHD FRM. Ratios of SMPS total and N30 number averages to SRI TEOM PM 2.5 are shown on second scale. 


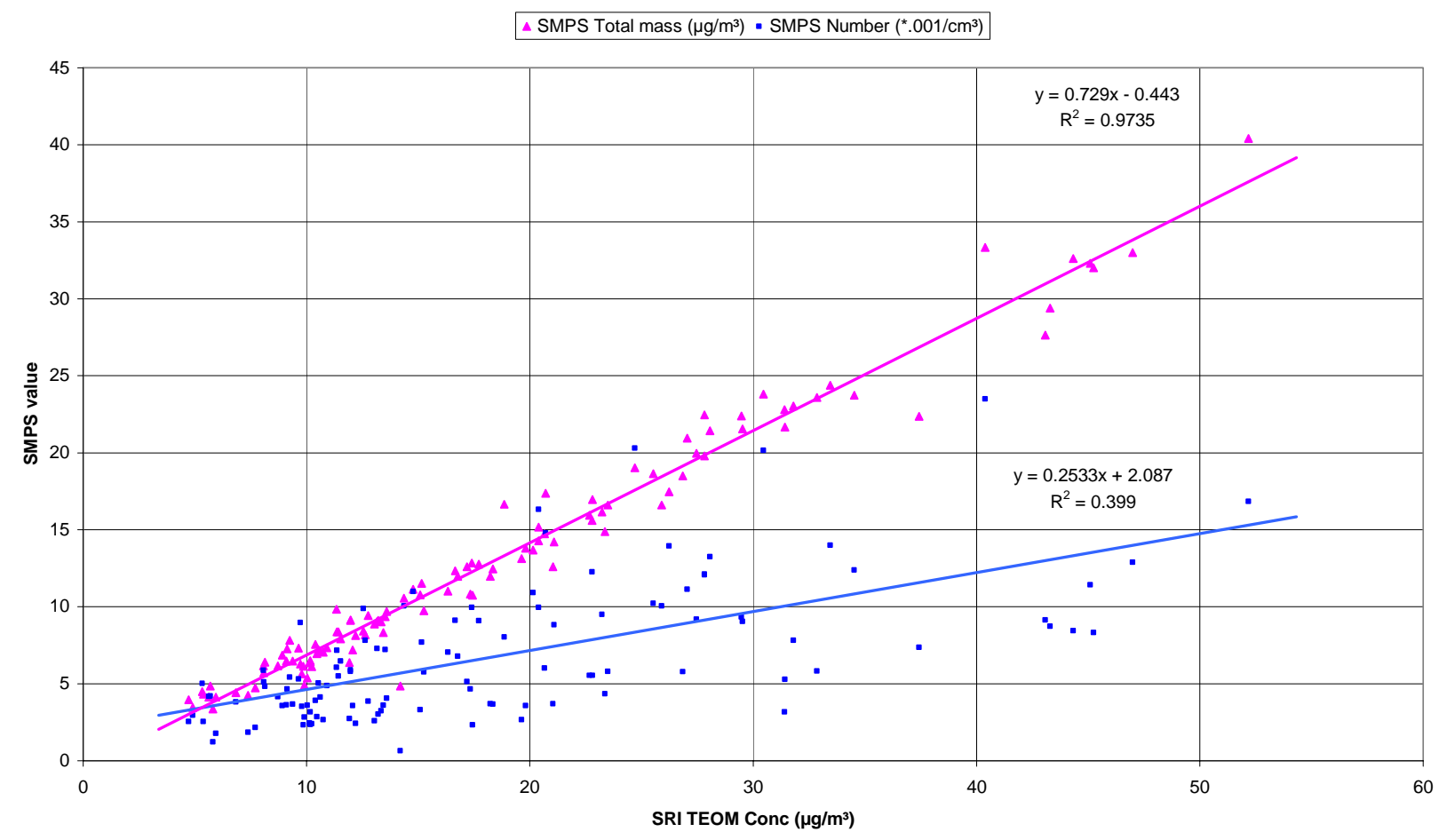

Figure 46. Comparison of daily average integrated SMPS mass and number concentrations to SRI TEOM PM P.. $_{2}$ over the year July, 2001 - June, 2002.

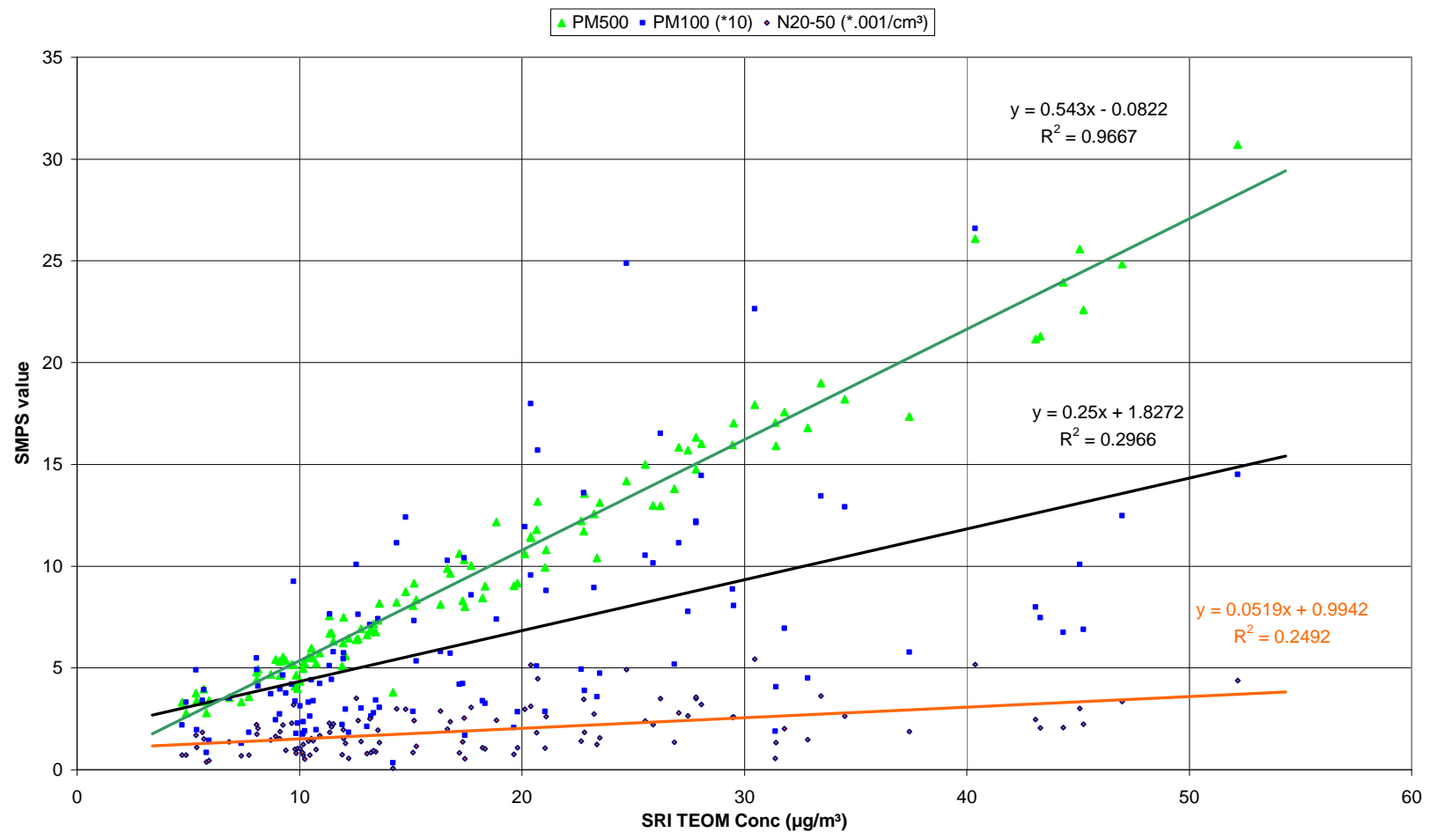

Figure 47. Comparison of daily average integrated SMPS size fractions to SRI TEOM $\mathbf{P M}_{2.5}$ over the year July, 2001 - June, 2002. 
Similar regressions were made for corresponding hourly averages of the SMPS and TEOM data over this time period. These are plotted in Figures 48-50. The same general trend with particle size is seen in the hourly average data; as seen in Figure 50, the smallest sizes are essentially uncorrelated with $\mathrm{PM}_{2.5}$ mass.

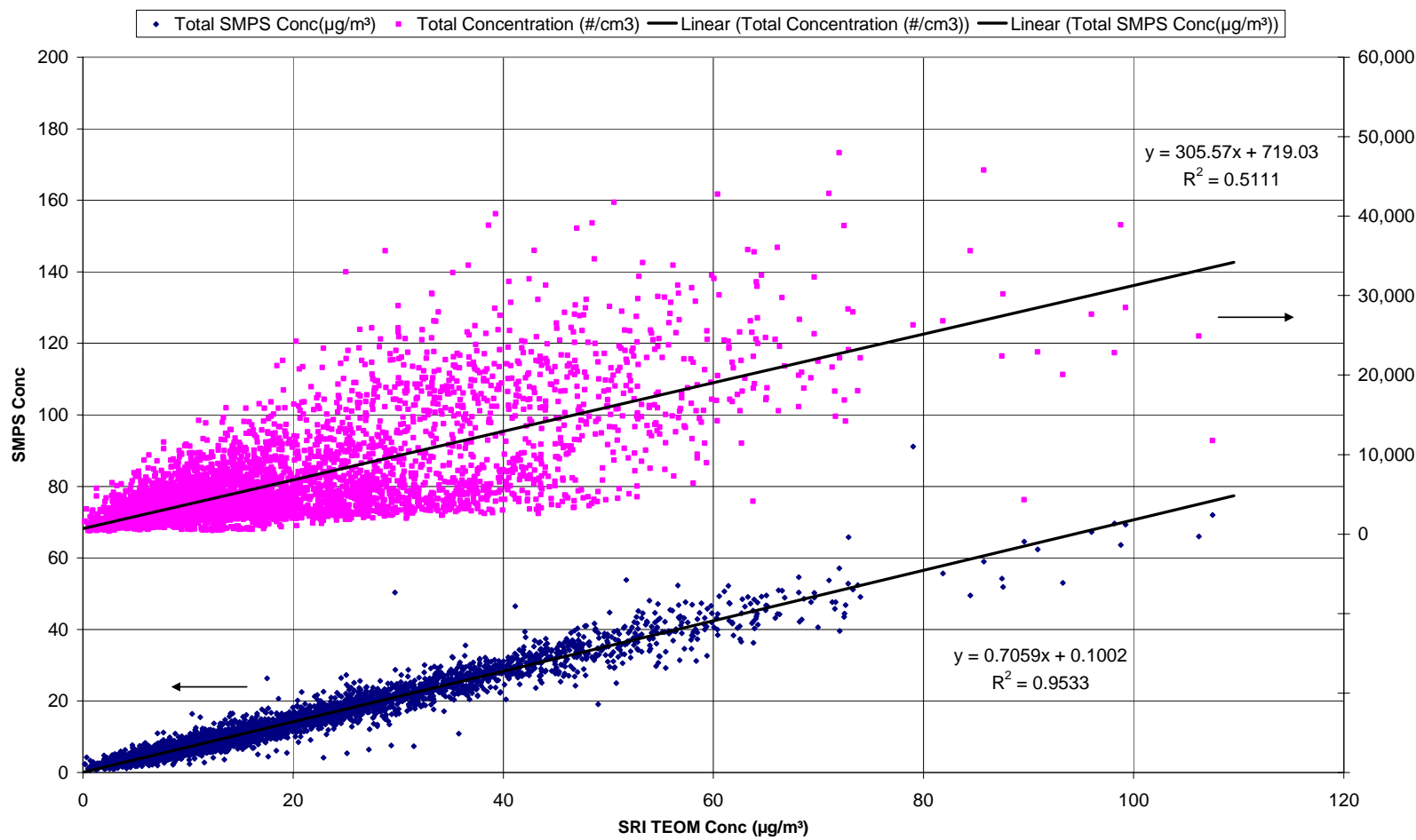

Figure 48. Comparison of hourly average integrated SMPS mass and number concentrations to SRI TEOM PM 2.5 over the year July, 2001 - June, 2002. 


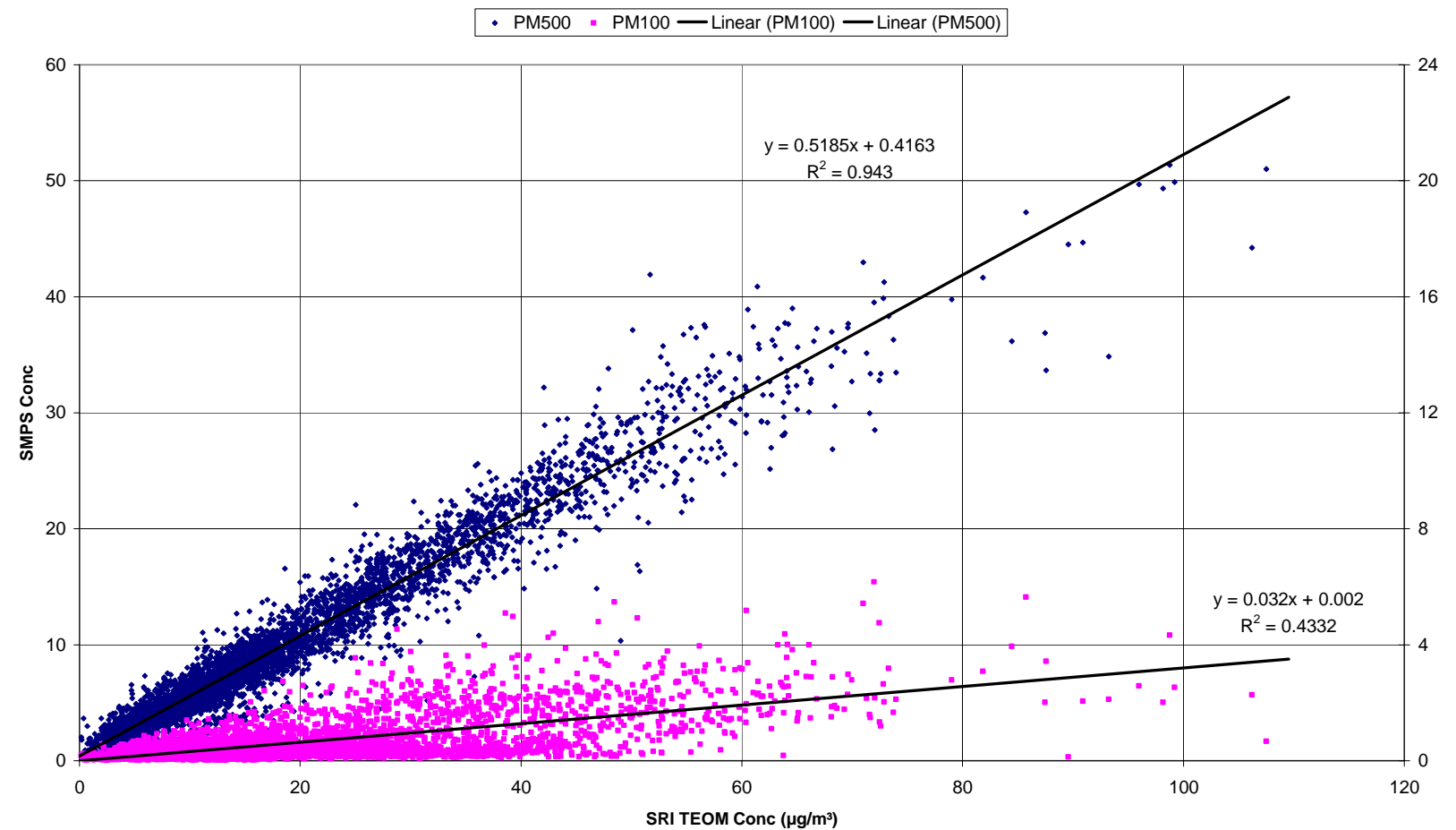

Figure 49. Comparison of hourly average integrated SMPS size fractions to SRI TEOM $\mathbf{P M}_{2.5}$ over the year July, 2001 - June, 2002.

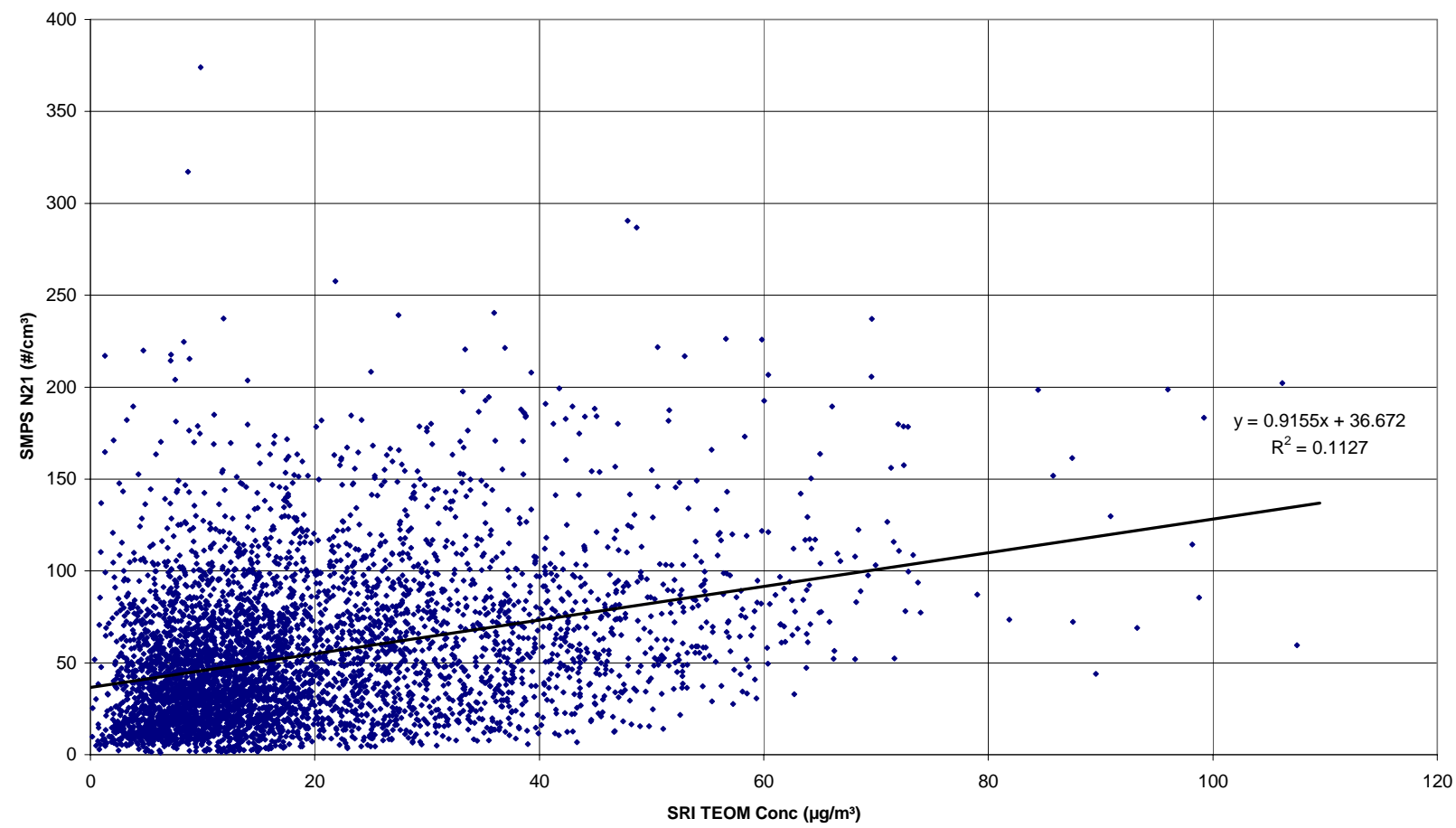

Figure 50. Comparison of hourly average SMPS number concentrations in lowest (21 $\mathbf{~ m m}$ midpoint) channel to PM $_{2.5}$ measured by SRI TEOM. 
Figure 51 shows the monthly variation of two integrated mass size fractions and measures of particle size over the project period. Averages are plotted for months with valid data for as few as $45 \%$ of available hours, so the values should be taken as indicative rather than quantitative.

The figure contains the both total integrated mass and the mass in the size range from $0.5-1 \mu \mathrm{m}$ physical diameter. This size band varies between 21 and $28 \%$ of the total mass, with an overall average value of $23 \%$. On the second axis of the figure are plotted the mass median and geometric mean diameter of the average monthly size distribution. Except for anomalous values in the month of October 2001, these average diameter values lie between 300 and $350 \mathrm{~nm}$. The mean diameters appear to vary seasonally, reaching the larger end of this range in the summer months and falling to lower values in the low - concentration winter months. As will be seen in the next slides, this shift represents a minor variation in the monthly average particle size distribution.

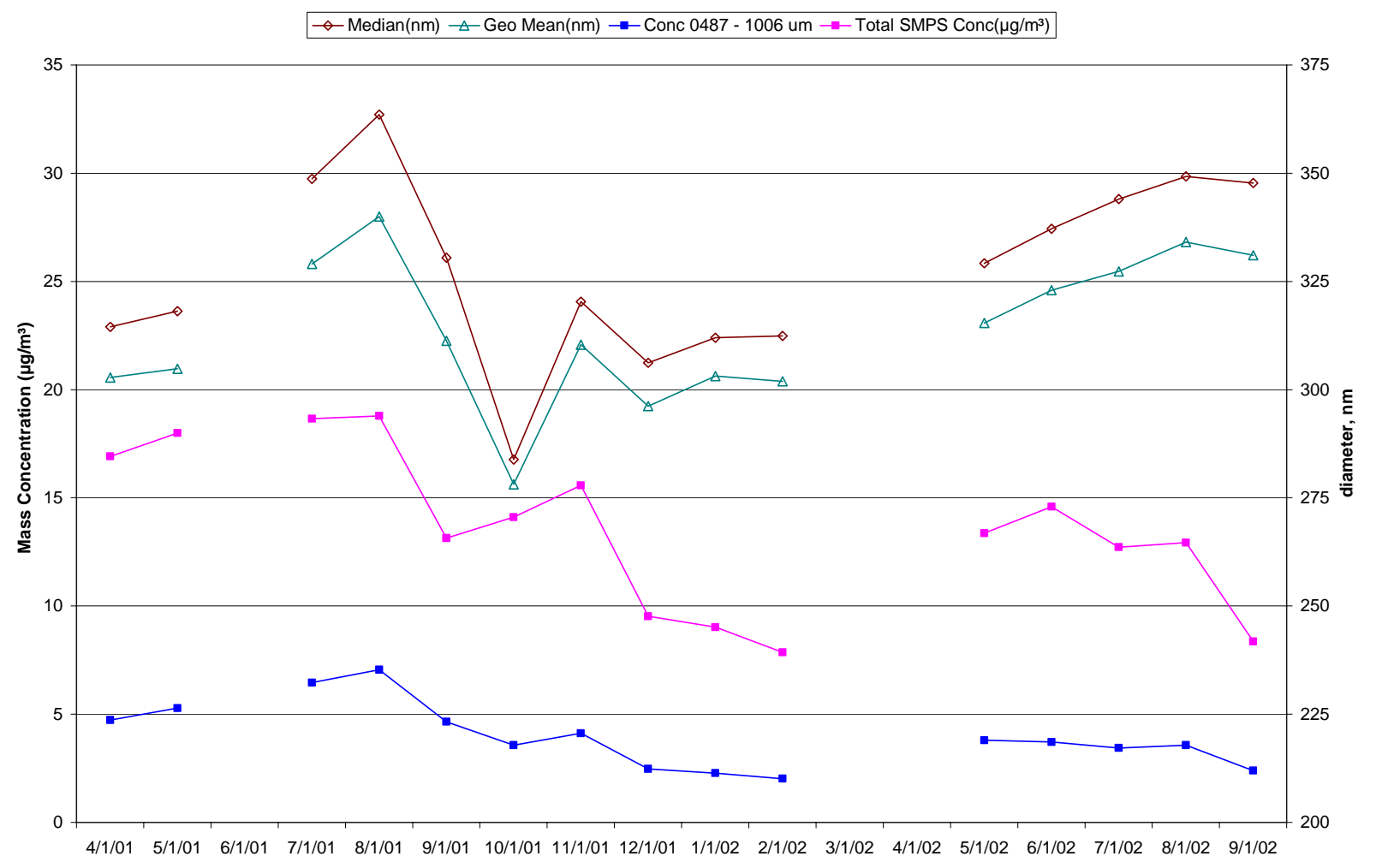

Figure 51. Monthly average SMPS concentrations and indicators of particle size for the project period.

Figure 52 is an overlay of monthly mean size distributions for the active sampling months of the project period. These are shown in differential concentration format with number and mass weighting, respectively, in Figures $52 \mathrm{~A}$ and B. While the overall concentration varies considerably from month to month, the overall shape of the distribution changes very little. This can be seen more clearly in Figures $53 \mathrm{~A}$ and B, which show the same distributions in relative form as cumulative percentage (of number or mass, respectively) less than the plot diameter. The distributions in the figure cluster tightly with either weighting. 

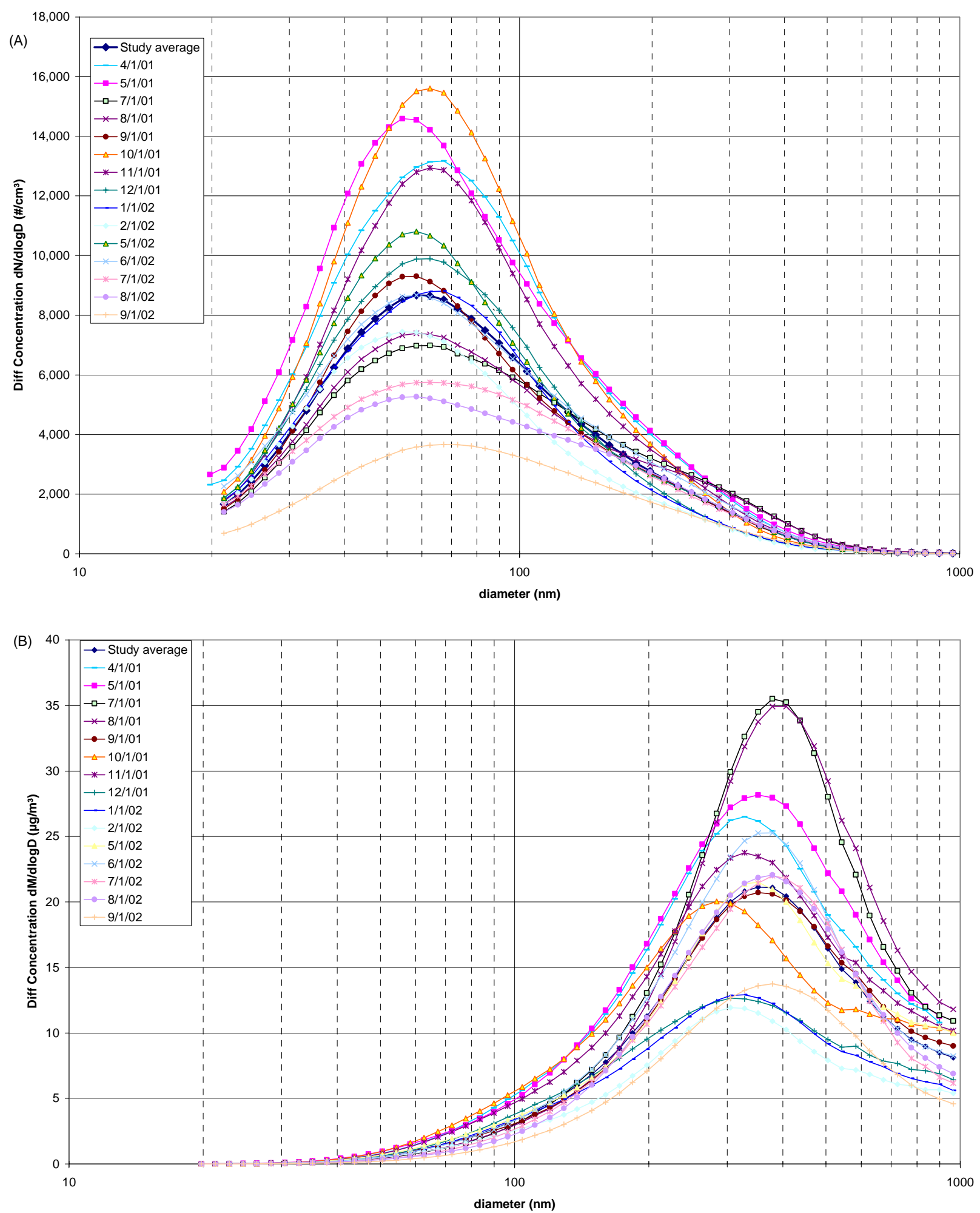

Figure 52. Average SMPS particle size distribution for project months in differential concentration format: (A) number weighted, (B) mass weighted. 

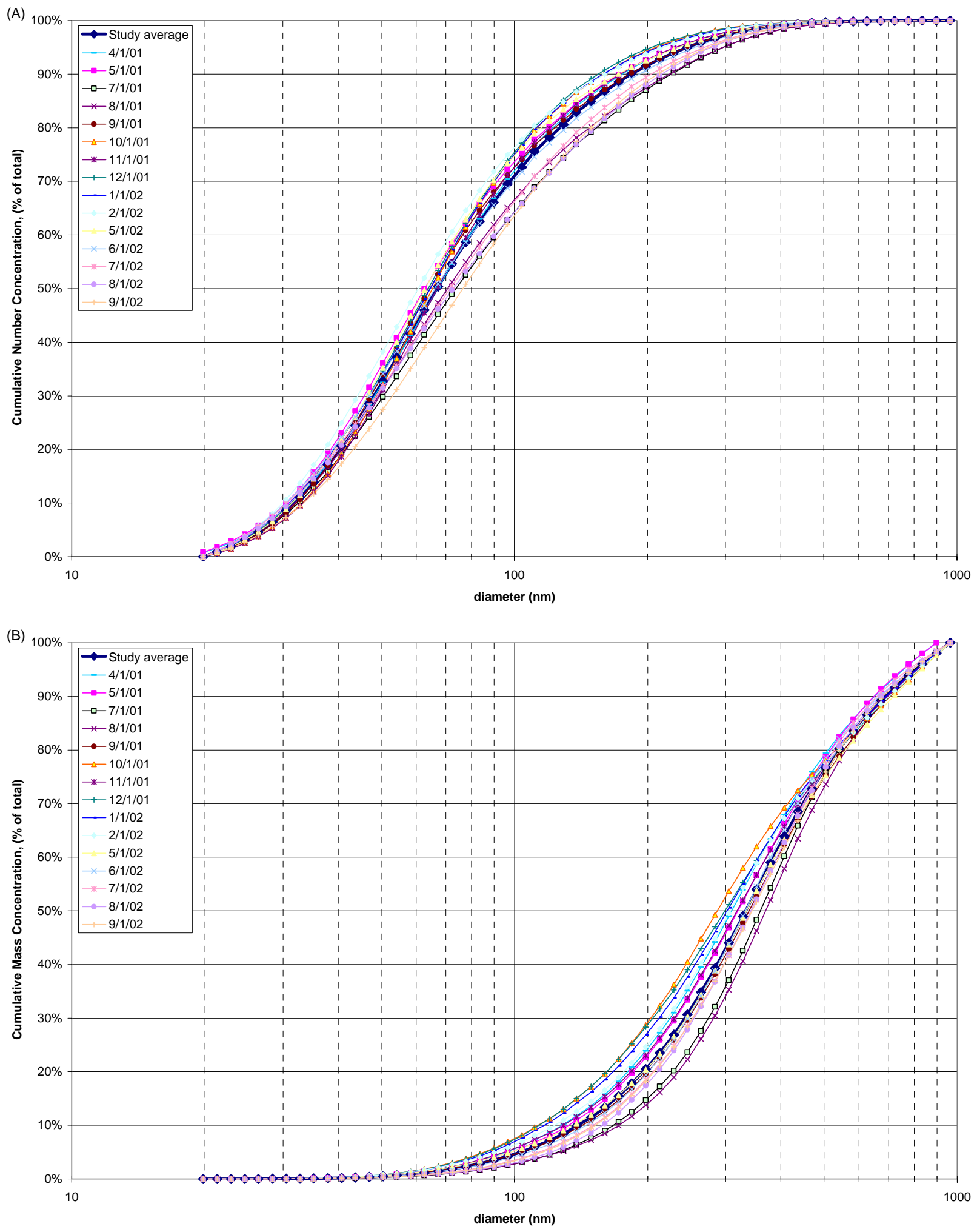

Figure 53. Average SMPS particle size distribution for project months in cumulative percent format: (A) number weighted, (B) mass weighted. 
As discussed in the time variation section above, individual submicron size fractions show different patterns of systematic variation over the average day period, leading to the conclusion that the shape of the overall size distribution should vary as a result. To illustrate this pattern, we generated composite hour of day average particle size distributions for comparison. These averages cover the period after the SMPS was installed in its final configuration on July 2, 2001. The earlier operating protocol gave spectra with slightly different boundaries which cause artifact discontinuities when averaged with size spectra from the main period. To avoid these effects, the earlier data were not included in the time of day composite averages. The size bands and mean diameters from these average distributions are plotted on Figure 54. The daily concentration pattern in the figure is repeated from the data previously shown in Figures 29 and 31, and show the typical daily features discussed there: the morning peak between 6 and 7 AM, followed by transitions to the daytime and overnight plateau levels. The mass median and mean diameter values vary systematically over a limited range, with minimum value immediately preceding the morning peak and maximum after the peak at the onset of the stable daytime concentration period.

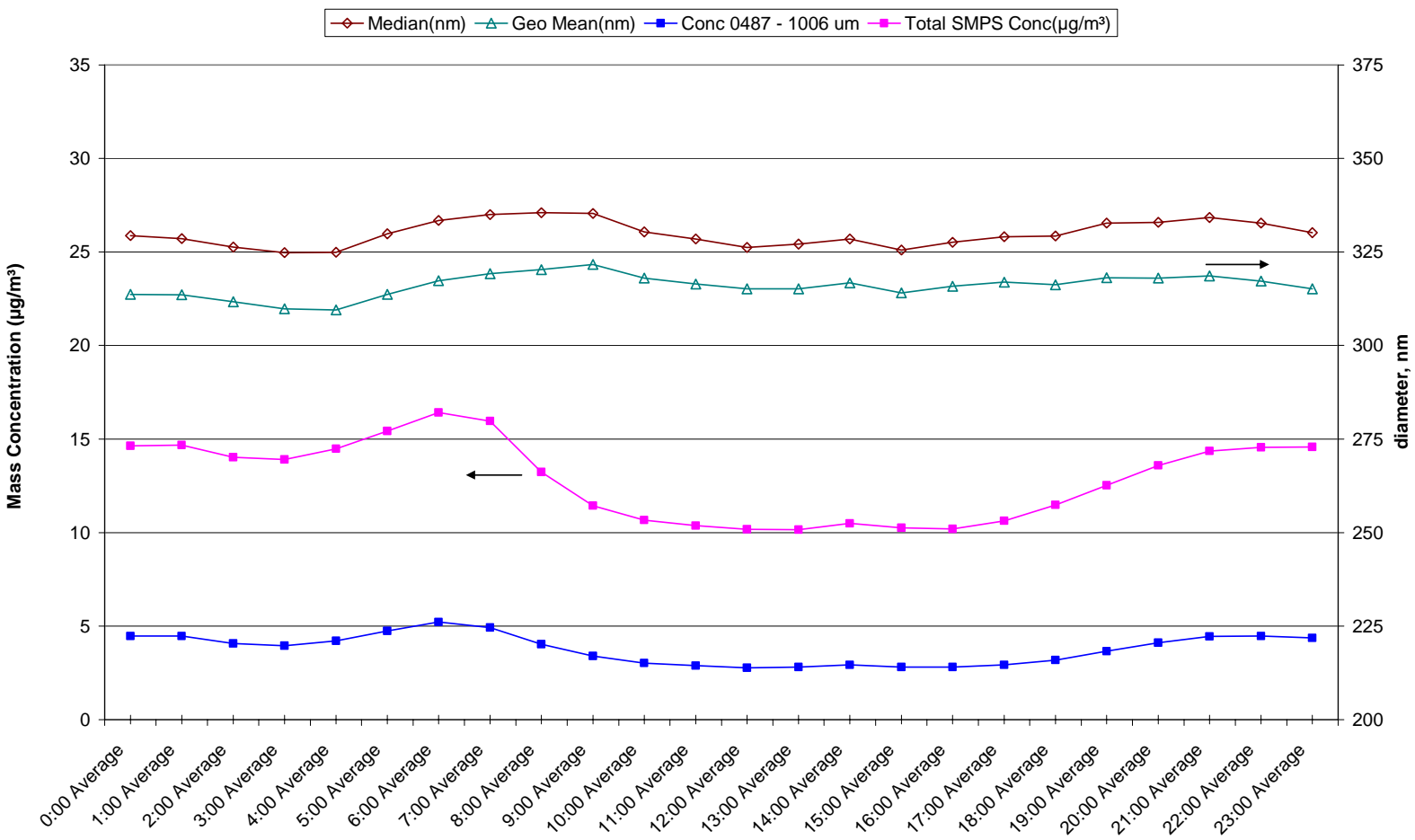

Figure 54. Hour of day average SMPS concentrations and indicators of particle size for the project period beginning July, 2001.

The corresponding hour of day size distributions are presented in Figures 55 and 56 . The presentation formats and diameter weighting for these figures are the same as the corresponding plots in Figures 52 and 53 above. The size distributions in these figures are even more uniform than the monthly distributions in the earlier set. The cumulative percent distributions in Figure $56 \mathrm{~A}$ and $\mathrm{B}$ are almost indistinguishable. The differential mass distributions form a family of non-intersecting, vertically displaced curves peaking in the same channel. There is a little more variety in the number weighted distributions, the peaks of which may differ as much as $20 \mathrm{~nm}$ from hour to hour. 

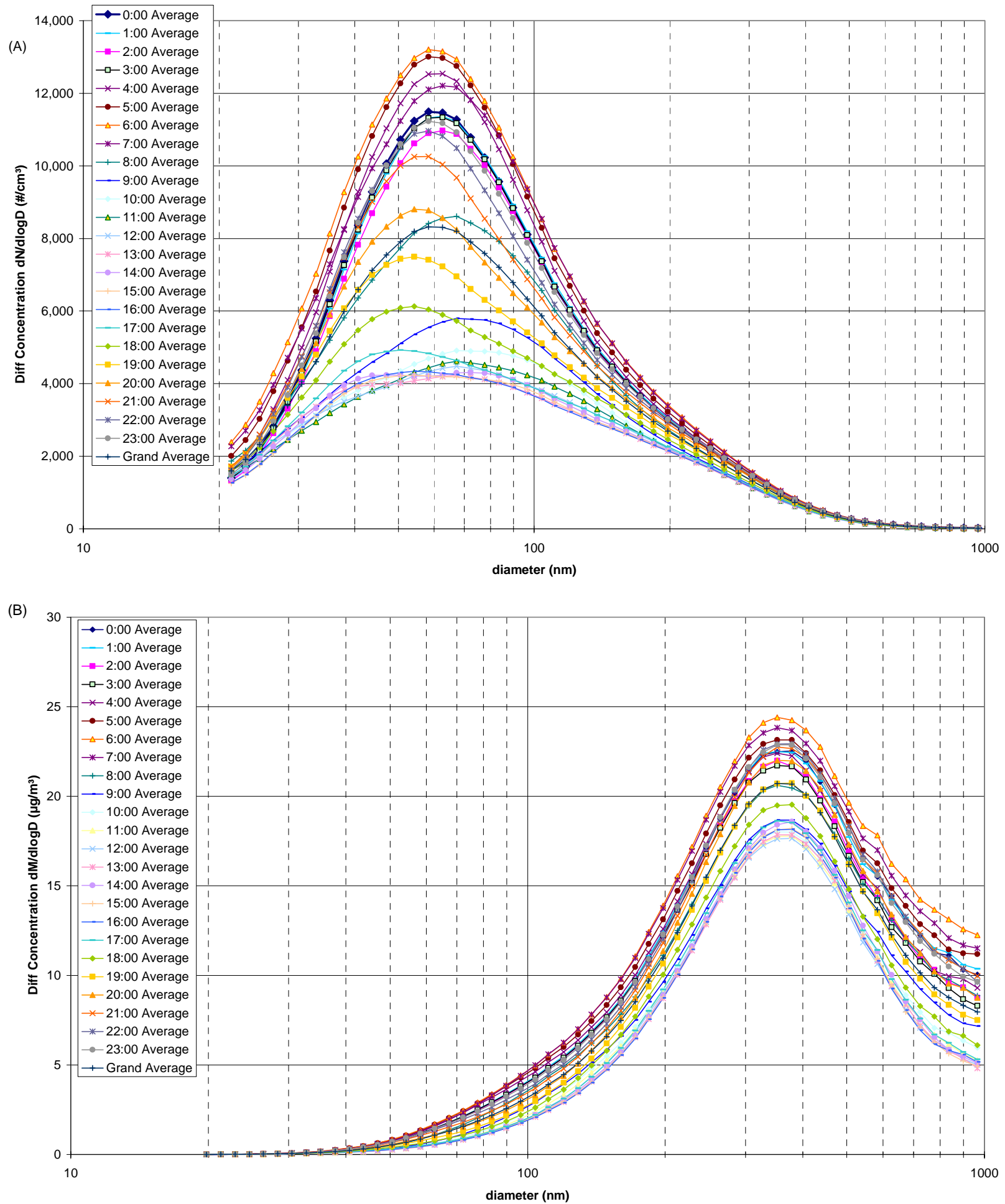

Figure 55. Hour of day average SMPS particle size distribution after July 1, 2001 in differential concentration format: (A) number weighted, (B) mass weighted. 

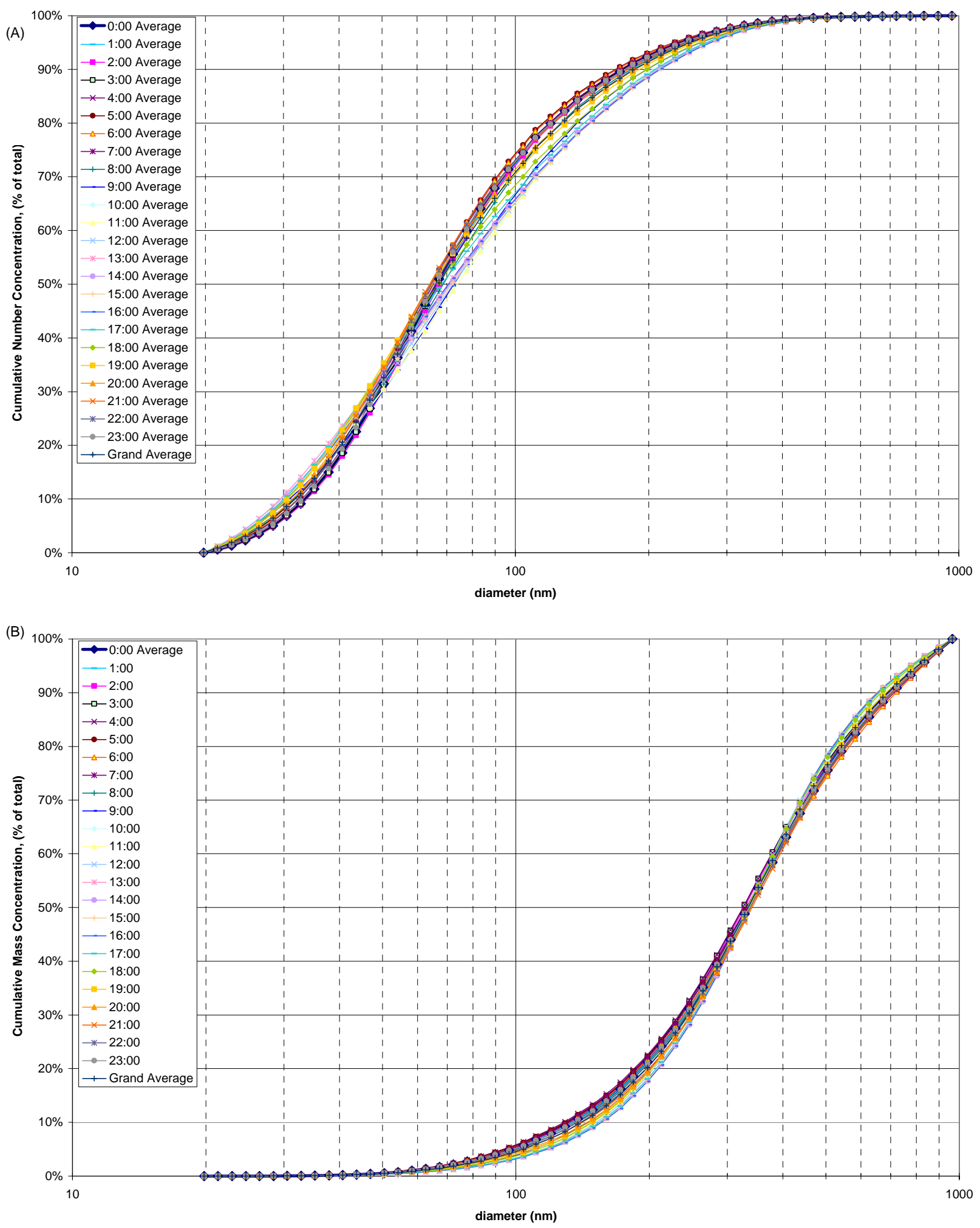

Figure 56. Hour of day average SMPS particle size distribution after July 1, 2001 in cumulative percent format: (A) number weighted, (B) mass weighted. 
This uniformity of size distribution is in part the result of "over-smoothing" in the composite averaging process, as short term or seasonal structure in the daily patterns are averaged out and lost. A more distinct daily pattern is preserved when constructing hour of day averages over a shorter period, such as a single season, or a single day. Figures 57-60 illustrate hour by hour size variations during a single summer day which are typical of the features seen in composite over the adjourning two month period. The day selected is $8 / 24 / 2001$, which is also the central day in the three day time series plot in Figure 20. As in the preceding figures, Figure 57 contains summary concentration and size parameters and the subsequent figures show hour-of-day average size distributions - in this case, averages over individual hours.

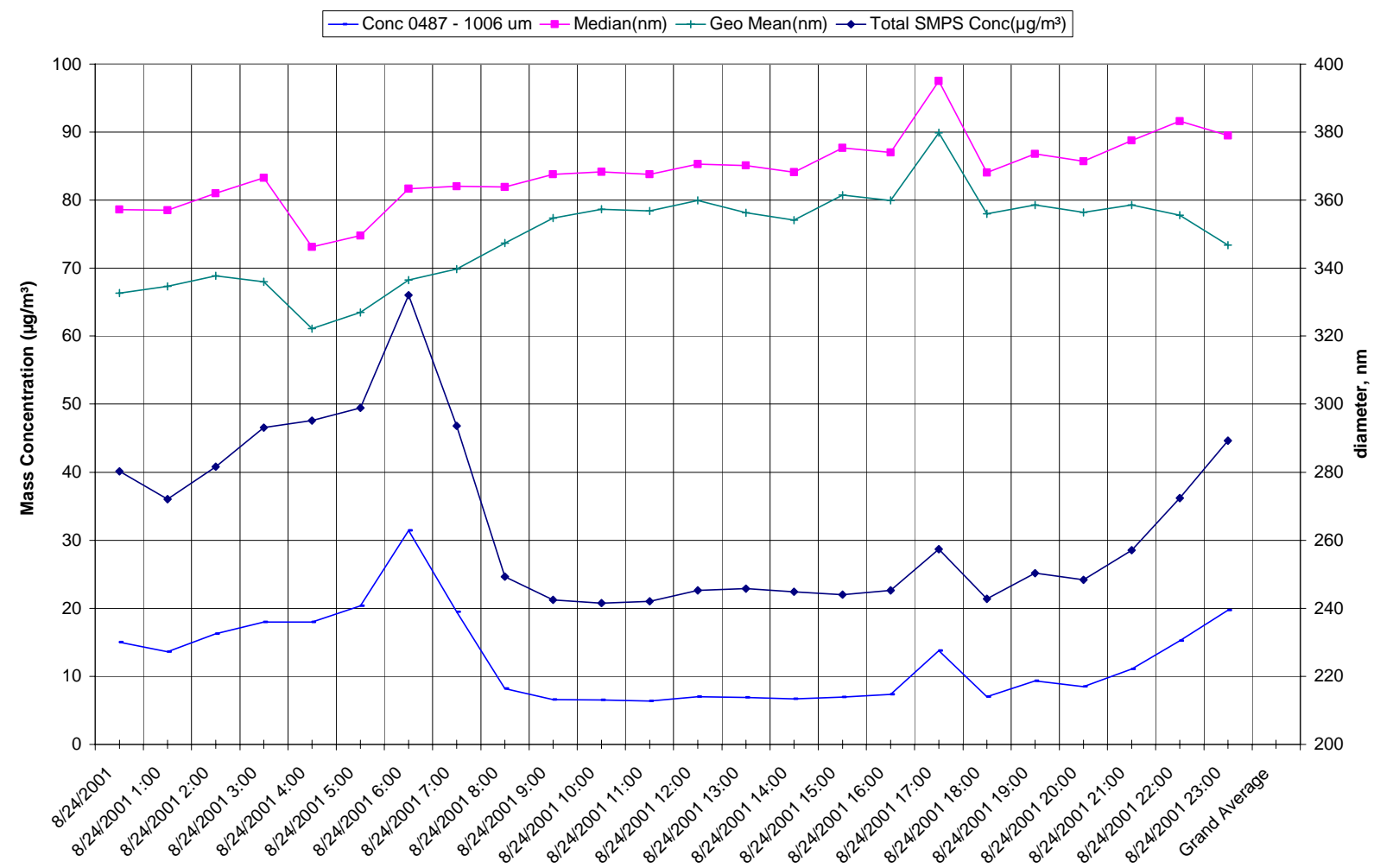

Figure 57. Hour of day average SMPS concentrations and indicators of particle size for August 24, 2001.

Figure 57, compared with the higher time resolution information in Figure 20, shows details that are typical of the summer period as well as individual events which would be lost in a composite multi-day average. The basic summer daily concentration pattern is evident, with morning peak, level daily concentrations, and a quasi-stable overnight concentration that actually is a composite of peaks from meandering plume impacts. This early morning structure is associated with changes in mean particle size, as is a narrow peak at 17:00 which is visible in the SMPS and nephelometer traces but not easily seen by the TEOMs or APS. 

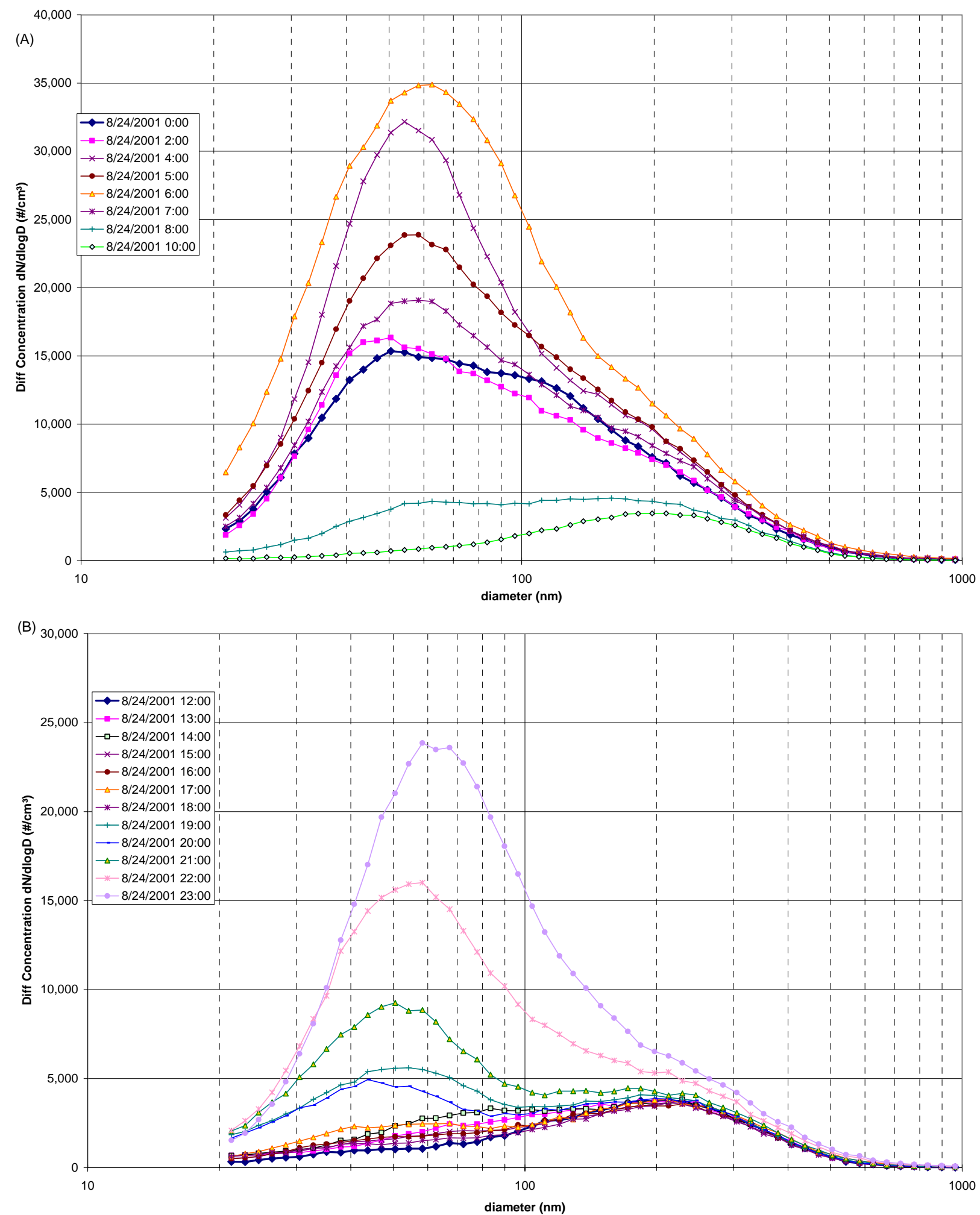

Figure 58. Hour of day average SMPS differential number weighted size distribution for 8/24/01: (A) hours before noon (B) hours after noon. 
Figures $58 \mathrm{~A}$ and B contain hourly average differential number size distributions over the course of the day, separated into morning and afternoon plots for visibility. The time sequence of the hourly spectra gives insight into the physical processes occurring during the day. In the early morning hours the plot is dominated by fluctuating levels of a fine aerosol with peak diameter around $55 \mathrm{~nm}$. These fine particles are typical of a freshly generated aerosol, whose source is necessarily nearby, as the local ventilation rate during these hours is low and variable; the observed concentration fluctuations are likely to be a combination of these variable factors. The morning peak at 6 AM contains increased concentrations at all sizes, which quickly decrease in number as the increased daytime atmospheric mixing begins. The $10 \mathrm{AM}$ size distribution is near the daily minimum, and spectra in Figure 58B for the remaining daytime hours remain near this range. The hours after $6 \mathrm{PM}$ are marked by an increasing ingrowth of an aerosol whose size distribution is broad and steadily shifts toward larger sizes as the day progresses. Figure 59 contains the same data on an expanded concentration scale, and reveals further details of the daytime pattern. First, all the distributions lie over an envelope size distribution that peaks at concentrations around 3500 particles $/ \mathrm{cm}^{3}$ at 200nm diameter. This residual size distribution is seen most consistently during the daylight hours when atmospheric dilution of the locally generated aerosol is greatest, and we interpret it as the best measure of the urban and regional background particle size distribution at the time. This aged background aerosol changes fairly slowly over this multiday stagnant period, and is combined with different amounts of locally generated aerosol mixtures which are also more variable in composition and size.

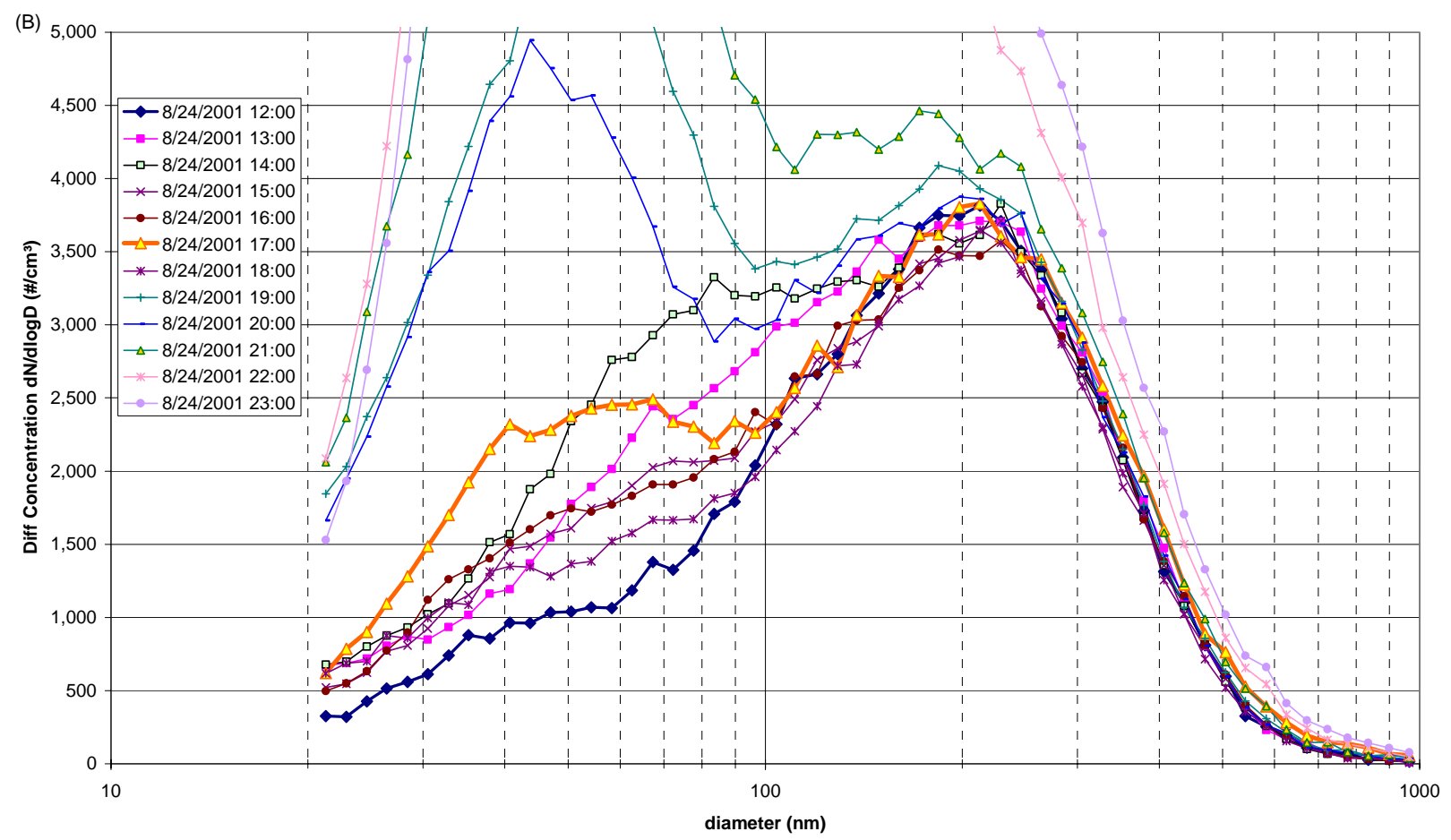

Figure 59. Expanded scale of SMPS differential number plot in Figure 58B, showing residual size distribution envelope.

At the expanded scale of Figure 59, we can see that during the daytime hours fluctuating amounts of local aerosol are added to the fine end of the size distribution. Except for the feature at 5 PM, which is highlighted in the plot, there is no notable component at larger sizes. 
Figure 60 displays the collection of hourly size distributions with mass concentration weighting. Due to the small number of samples in the average, there is considerable statistical scatter in the upper end of the size distributions, where the number of sampled particles is low. The 5 PM size distribution is again highlighted, showing the large particle portion of this component. Also visible is the large particle portions of the enhanced evening and overnight size distributions and of the 6 AM morning peak. The 6 AM distribution is enhanced at all sizes, but especially for particle sizes larger than $0.5 \mu \mathrm{m}$. Otherwise, the hourly average spectra show the "stacking" effect that is seen in the composite hour of day average distributions. All distributions follow the outline of the residual aerosol size distribution, but are incremented by varying contributions of locally generated aerosol. Above $100 \mathrm{~nm}$, the size distribution of these hourly increments is similar in shape, having a peak near $360 \mathrm{~nm}$ the residual aerosol but with slightly more "tailing" to large diameters. This similarity of the net local increment size distribution and the urban/regional background accounts for the overall time stability of the measured submicron particle size spectrum.

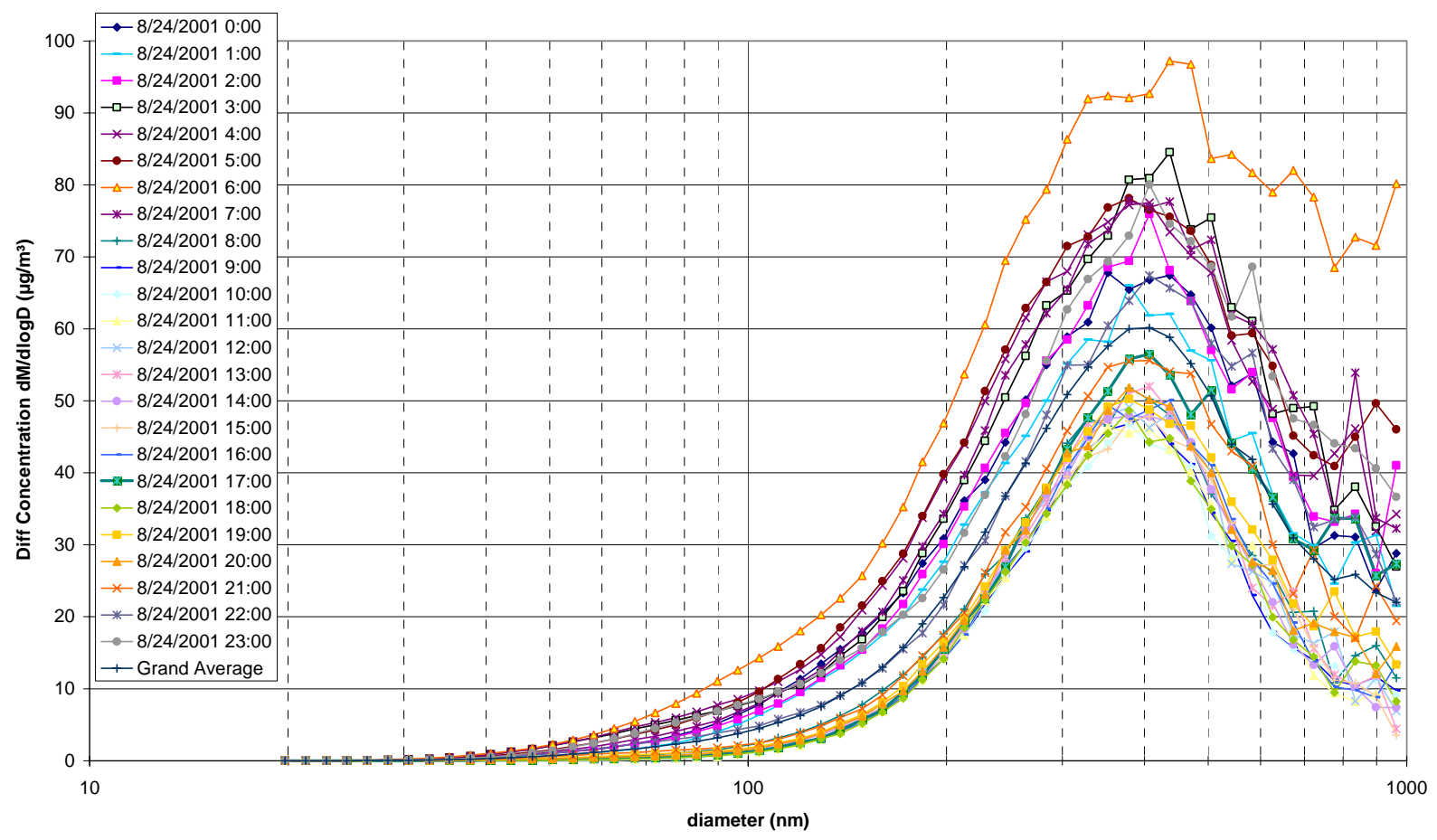

Figure 60. Hour of day average mass weighted SMPS particle size distribution for 8/24/01.

$\underline{\text { PM fractions and size distribution }>1 \mu \mathrm{m} \text { : APS data }}$

Like the SMPS, the APS is not optimized for precise measurement of overall particle mass concentration. Nonetheless, for this device also it is instructive to compare integrated size spectra with the contemporary particulate measurements of from the TEOM or FRM monitors at the site. For the APS the relevant comparisons are of $\mathrm{PM}_{2.5}$ with all measurable mass below $2.5 \mu \mathrm{m}$ aerodynamic diameter, and PMc with the corresponding APS size fraction. These comparisons are shown in Figures 61 and 62. 


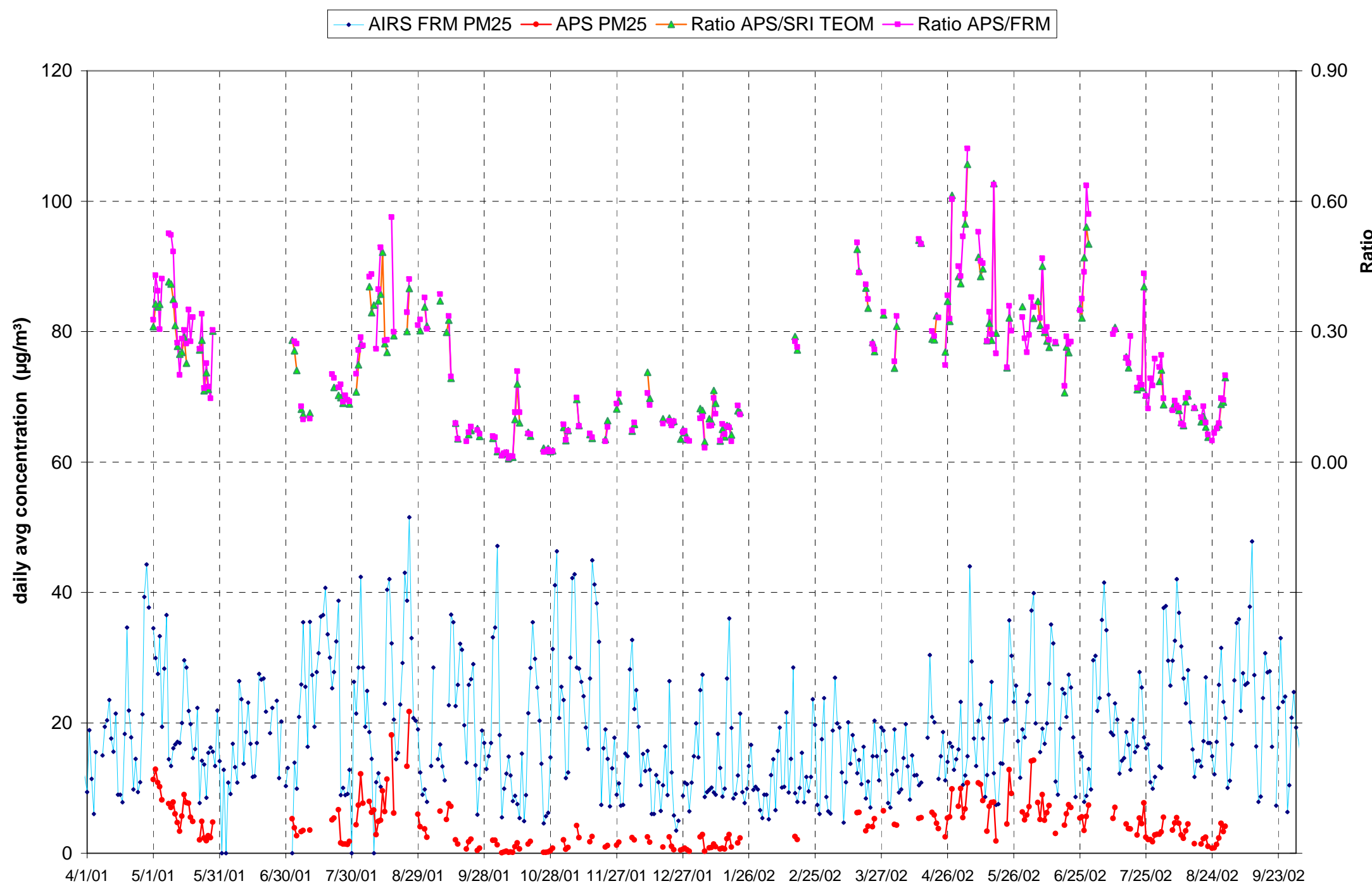

Figure 61. Daily average integrated APS mass below $2.5 \mu \mathrm{m}$ plotted with PM$_{2.5}$ concentration from JCHD FRM. Ratios of APS mass averages to PM $_{2.5}$ from FRM and SRI TEOM are shown on second scale. 


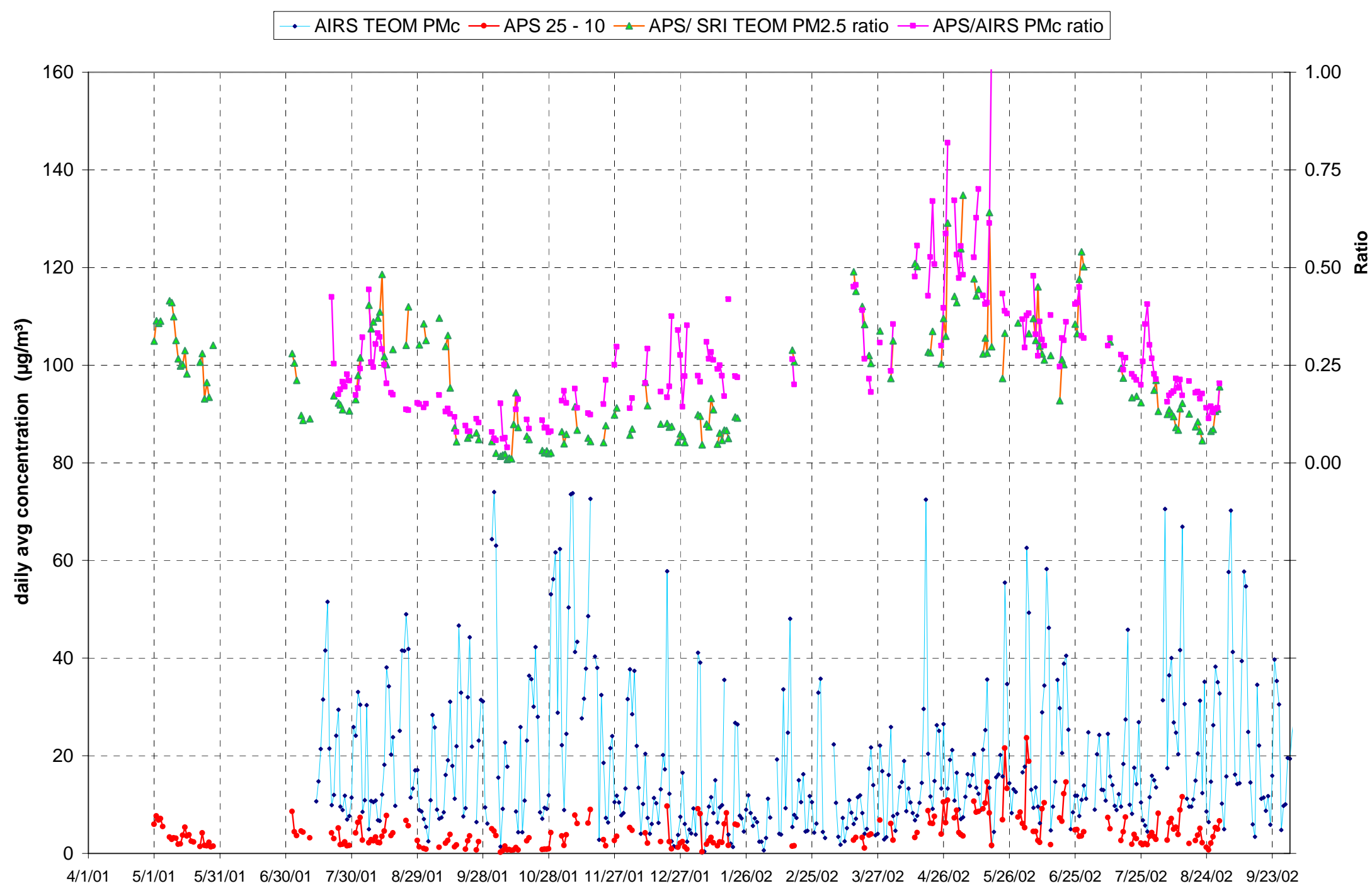

Figure 62. Daily average integrated APS mass between 2.5 and $10 \mu \mathrm{m}$ plotted with PMc calculated from JCHD TEOMs. Ratios of APS mass averages to PMc and SRI TEOM PM 2.5 are shown on second scale. 
Figure 61 shows daily average APS integrated particle size distribution data up to $2.5 \mu \mathrm{m}$, and the ratio of this quantity to the daily $\mathrm{PM}_{2.5}$ measured by the JCHD FRM and the SRI PM 2.5 TEOM. For comparison purposes, the FRM concentrations are plotted on the same scale with its APS equivalent. The figure is similar to the corresponding SMPS plot shown in Figure 44. As in that figure, daily averages are calculated for the TEOM and APS only for days having more than 21 valid measurement hours. This requirement led to elimination of several incomplete days as well as more extended gaps when the instrument was not present. It is evident from the figure that the ratio of the APS concentration to the $\mathrm{PM}_{2.5}$ reference instruments is quite variable. For some periods this can be explained in terms of instrumental operating factors; for others there is no clear explanation. As described in the experimental section, July and August, 2001, which had a sustained high ratio to the reference $\mathrm{PM}_{2.5}$, also represent a period of high dead time corrections. The abrupt drop in instrument response beginning on 9/13/01 is evident in the figure, as are later shifts in October that appear to relate to partial flow obstruction before the nozzle plugged on 10/31/01. Although the flow path was restored and calibration verified after November 2, the low efficiency persisted until the instrument was removed in January, 2002 for upgrade. After the upgraded 3321 unit was installed in February, 2002, it operated at sustained high response levels through June. Beginning in July, 2002 a gradual degradation of response can be noted in the figure; though the cause for this is unclear. At the end of August, the instrument developed a high level of artifact counts, and sampling was terminated. In summary, both versions of the APS experienced short-term scatter and long-term drift in relative response to ambient $\mathrm{PM}_{2.5}$ aerosol. The magnitude of this variation is shown in the pairwise comparison plot in Figure 63 below.

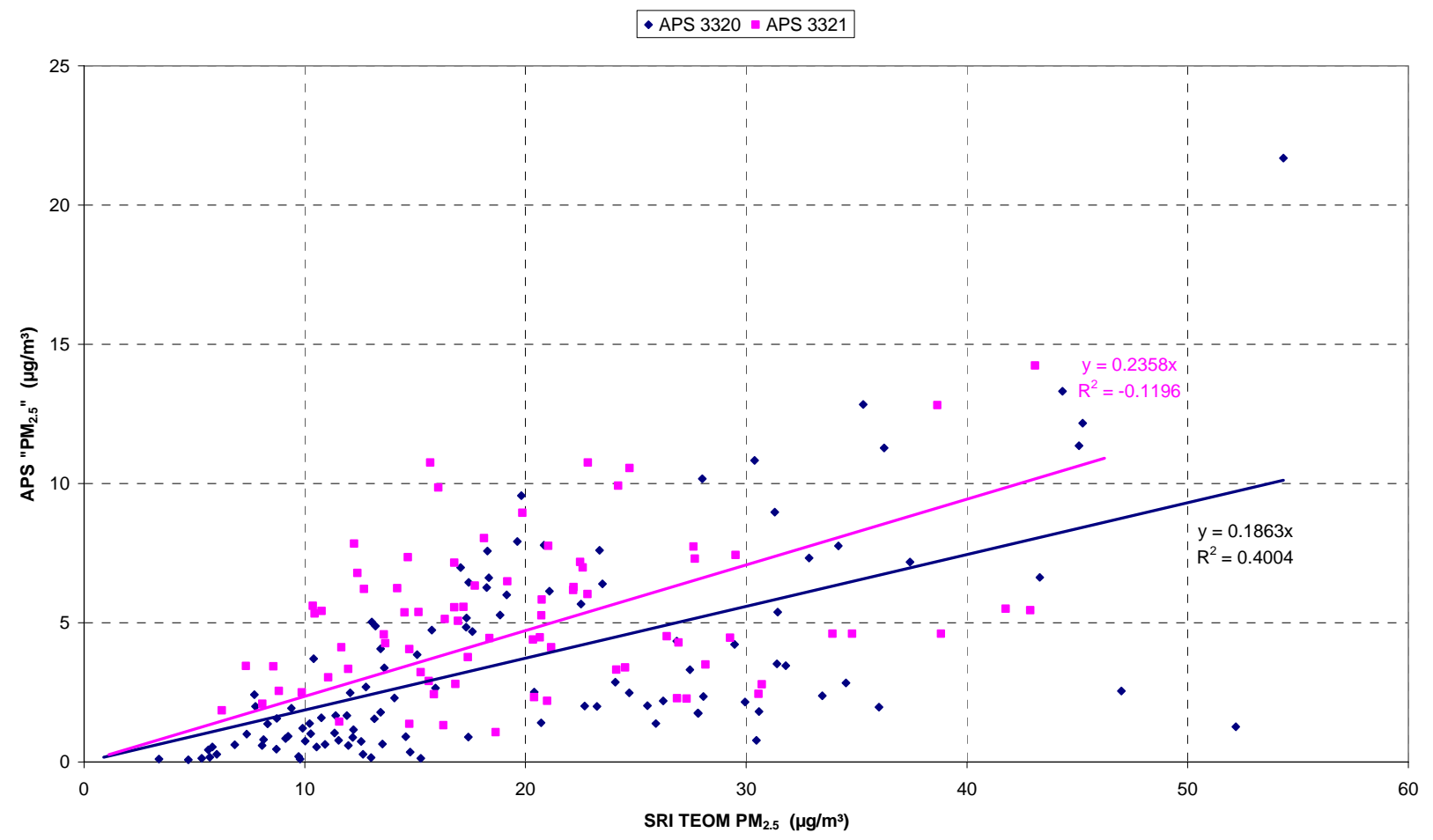

Figure 63. Comparison of daily average APS integrated mass concentrations $<2.5 \mu m$ to PM $_{2.5}$ from SRI TEOM before and after APS upgrade. 
In Figure 63, the daily average APS integrated " $\mathrm{PM}_{2.5}$ " particle size distribution for each instrument model is plotted separately against the corresponding TEOM daily average. While the figure contains regression trend lines for each device, these represent medians to the long term drift of the ratio rather than meaningful fits to the data. Though the scatter bands overlap considerably, the figure shows the generally higher response of the 3321 configuration. During periods with the highest response the $\mathrm{PM}_{2.5}$ ratio approaches 0.5 , which is probably physically realistic in view of the significant mass fraction smaller than $0.5 \mu \mathrm{m}$ which the APS does not measure. Clearly neither device is useful as a surrogate indicator of $\mathrm{PM}_{2.5}$.

The particle size range of greater importance for the APS is the coarse particle fraction, defined here as $2.5-10 \mu \mathrm{m}$ aerodynamic diameter. This fraction lies fully within the operating range of the APS, although transport losses and sampling biases can affect the accuracy of the measurements for the larger sizes. The comparison time series plot in Figure 62 and the pairwise plot in Figure 64 address the accuracy of the integrated APS size distribution for this size range. As before, the reference value for PMc is calculated from the difference of hourly averages from the Jefferson County $\mathrm{PM}_{10}$ and $\mathrm{PM}_{2.5}$ TEOM samplers. In Figure 62 the $\mathrm{PM}_{2.5}$ ratio is again plotted for comparison along with the ratio of the PMc measures.

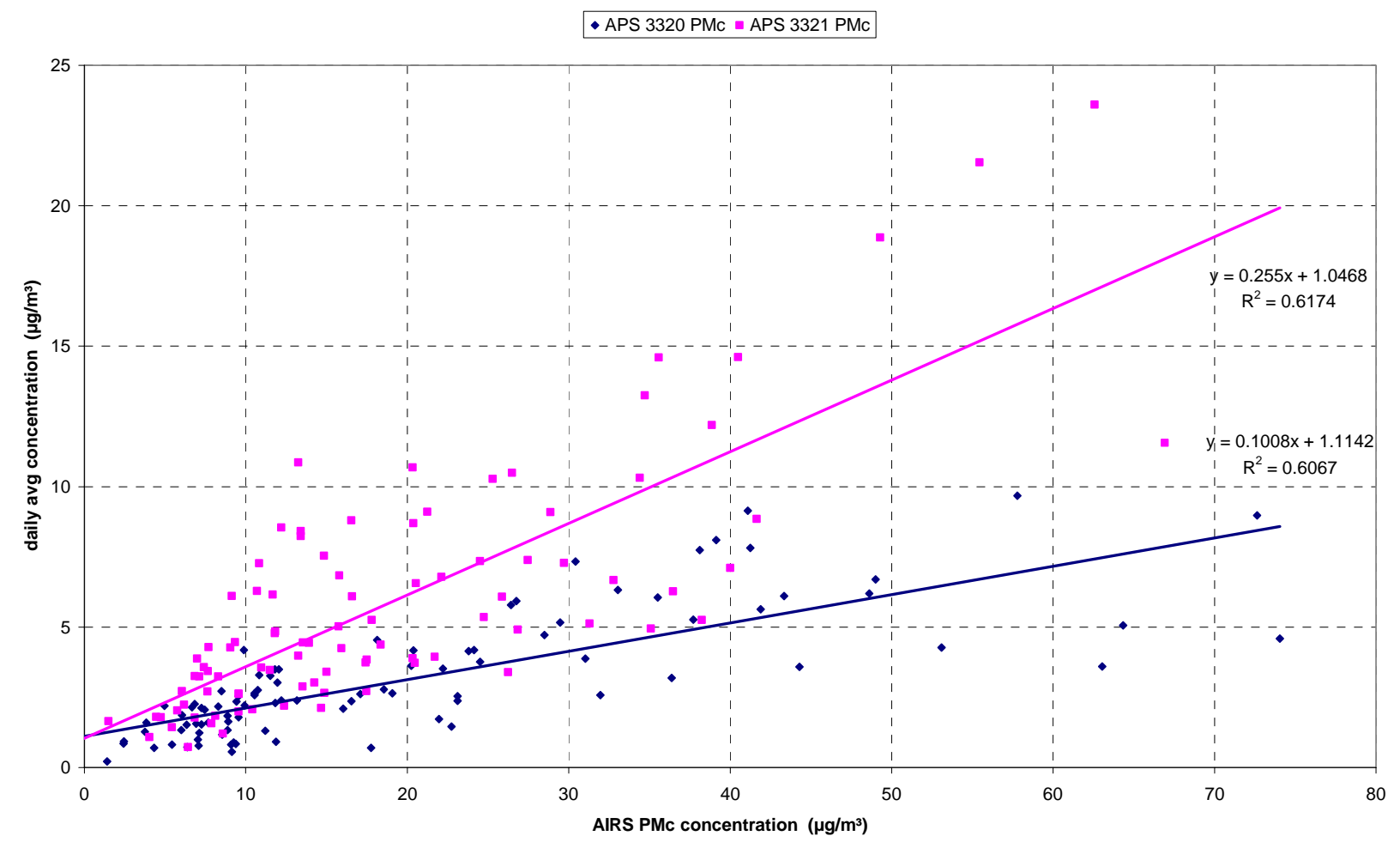

Figure 64. Comparison of daily average integrated PMc from APS before and after upgrade to PMc concentrations calculated from JCHD TEOMs.

The comparison plots in Figures 62 and 64 reveal some interesting trends. First, the APS averages are significantly lower than the reference PMc at all times. The aggregate response ratios of the 3321 and 3320 are 29\% and 13\%, respectively, of the TEOM PMc. It is not clear whether this low response is due to inefficiencies in sampling and transport line losses as well as instrument response. Second, the behavior of the two APS models is markedly different for this size fraction. Inspection of the ratios after January 2002 in Figure 62 confirms that the PMc ratio 
for the 3321 is highly correlated with the $\mathrm{PM}_{2.5}$ ratio, and presumably reflects variations in overall instrument response for all sizes. In the original 3320 configuration, the PMc ratio and $\mathrm{PM}_{2.5}$ ratio appear to drift independently. In mid-August, 2001, the PMc ratio drops abruptly while the $\mathrm{PM}_{2.5}$ ratio remains high, then does not follow the abrupt drop in the $\mathrm{PM}_{2.5}$ ratio in mid-September. In short, both configurations of the instrument experienced long-term changes in response to PMc that appear as excessive scatter in plots like Figure 64. Short-term correlations with PMc appear to be better, and in the case of the 3321 configuration, more predictable.

The changing response of the APS raises the question of the reproducibility of the measured size distributions over time. To assess this, monthly average size distributions were computed for the APS in the same manner as described above for the SMPS. The results of this process are shown in Figures 65 through 67 below. Figure 65A and B present particle size distributions for the 3320 and 3321 models in differential mass format for particle aerodynamic equivalent diameters up to $10 \mu \mathrm{m}$. For ease in comparing size distributions for different months, the same data are plotted in cumulative percent mass format in Figure 66A and B, and in a normalized differential format in Figure 67A and B, where all distributions are scaled by the size-integrated mean for that monthly average.

In each figure pair it is apparent that the APS 3320 monthly size distributions differ dramatically, while the APS 3321 size distributions have at least a similar shape. In these distributions, as shown in Figure 65B, the major feature is the submicron mode, which is underestimated in the figure (the rollover in the distribution at about $650 \mathrm{~nm}$ is an artifact of the rapid decrease in response for diameter smaller than this range - the SMPS data show that the real peak of this particle mode falls between $200-400 \mathrm{~nm}$ ). The size distribution has the typical minimum between 1 and $2 \mu \mathrm{m}$, then one or two coarse particle modes between 2.5 and $10 \mu \mathrm{m}$. For most of the months the first mode near $3 \mu \mathrm{m}$ is slightly higher than the second, near $7 \mu \mathrm{m}$. The Model 3320 size distribution for May, 2001 most resembles this pattern, with the $7 \mu \mathrm{m}$ mode pushed above the $10 \mu \mathrm{m}$ boundary of the plot. Most of the other monthly distributions in Figure 65A follow different patterns, some of which are physically unrealistic. After May, the submicron mode decreases and is replaced by a phantom mode at $1 \mu \mathrm{m}$. This apparently results from an instrumental artifact that involves mismeasurement of the particle time of flight. After the acceleration nozzle was cleaned, a submicron mode reappeared in the November size distribution, but again decreased in size over the final two months. In addition to these suspect features at the small end of the size spectrum, the size distributions show an increasing background contribution of apparent particles at the large particle end. Since a known artifact of the original 3320 geometry is a background of "ghost" large particles, this size region is also suspect. We cannot be optimistic that Model 3320 size distributions for any month after May are accurate in more than a general sense.

It is of note that the Model 3320 monthly average size distributions also show a progressive trend toward reduced submicron mode height combined with increased counts assigned to particles larger than $5 \mu \mathrm{m}$. This can best be seen in Figure 68B by the progression from February and March, 2002 through the cluster of similar distributions in the following months, to the average for October. While this could be a real trend in particle size, it could also be a sign of slow degradation of instrument performance. 

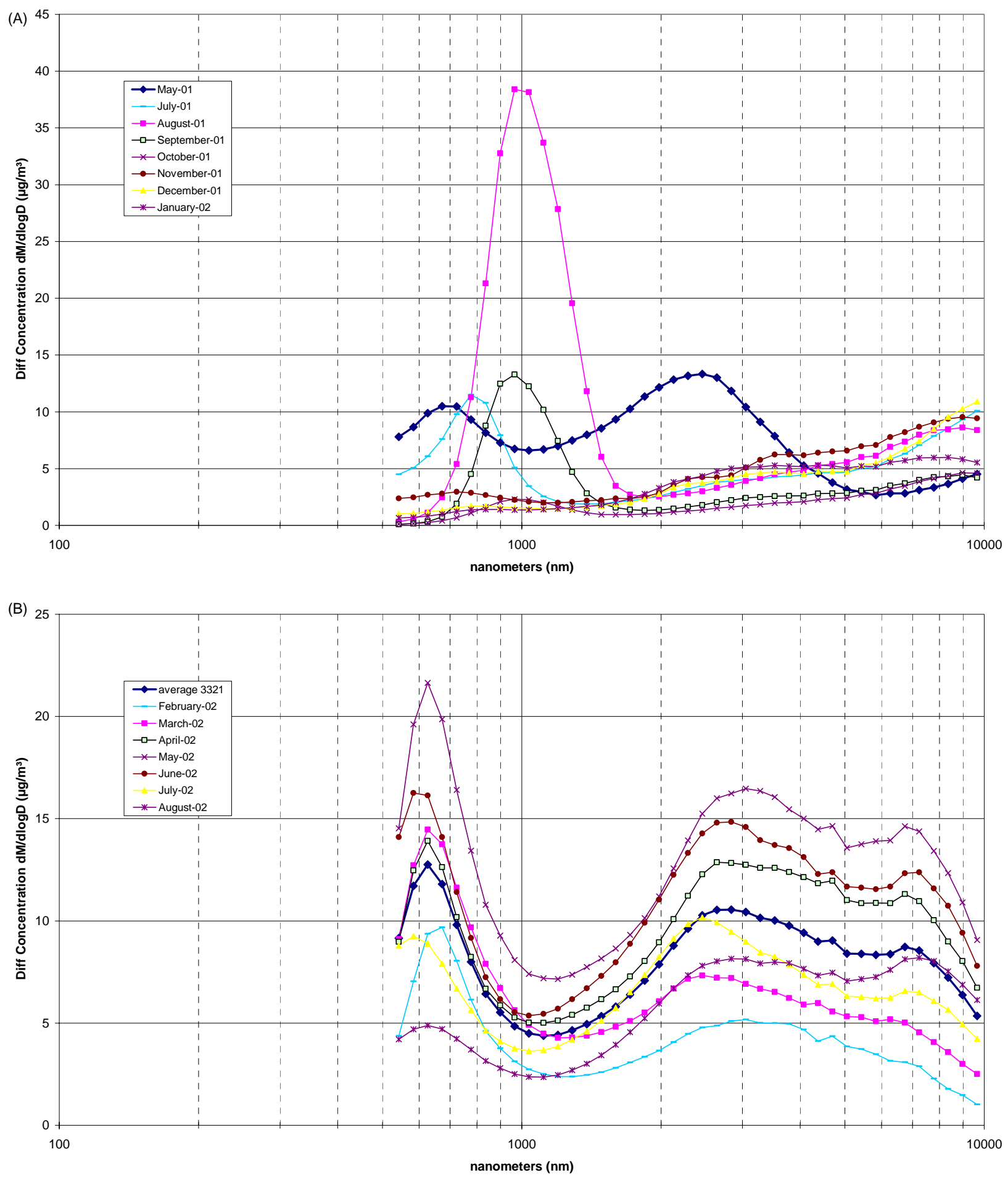

Figure 65. Average monthly differential mass weighted particle size distribution for APS instruments: (A) APS 3320, (B) upgraded APS 3321. 

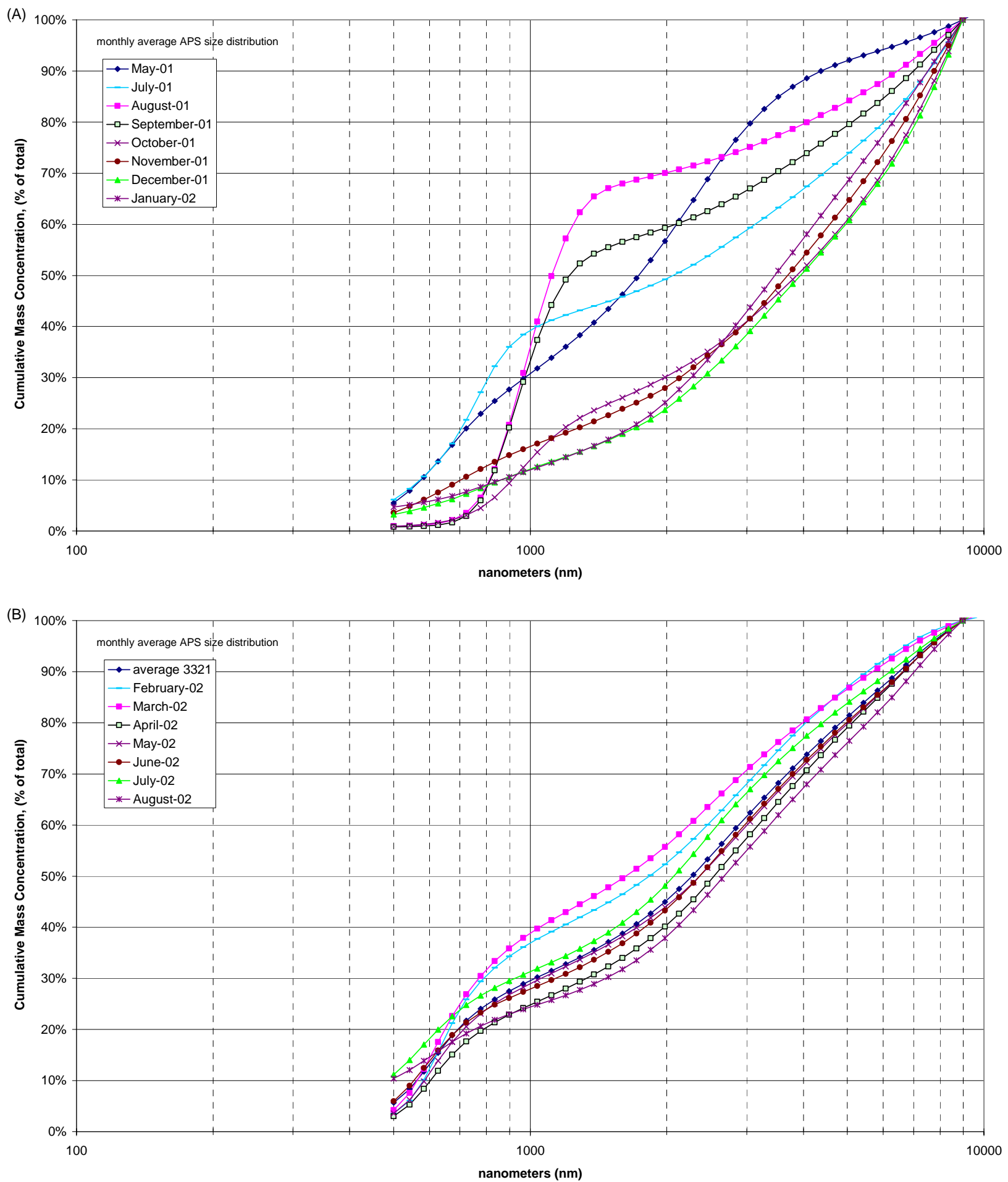

Figure 66. Average monthly cumulative percent mass weighted particle size distribution for APS instruments: (A) APS 3320, (B) upgraded APS 3321. 

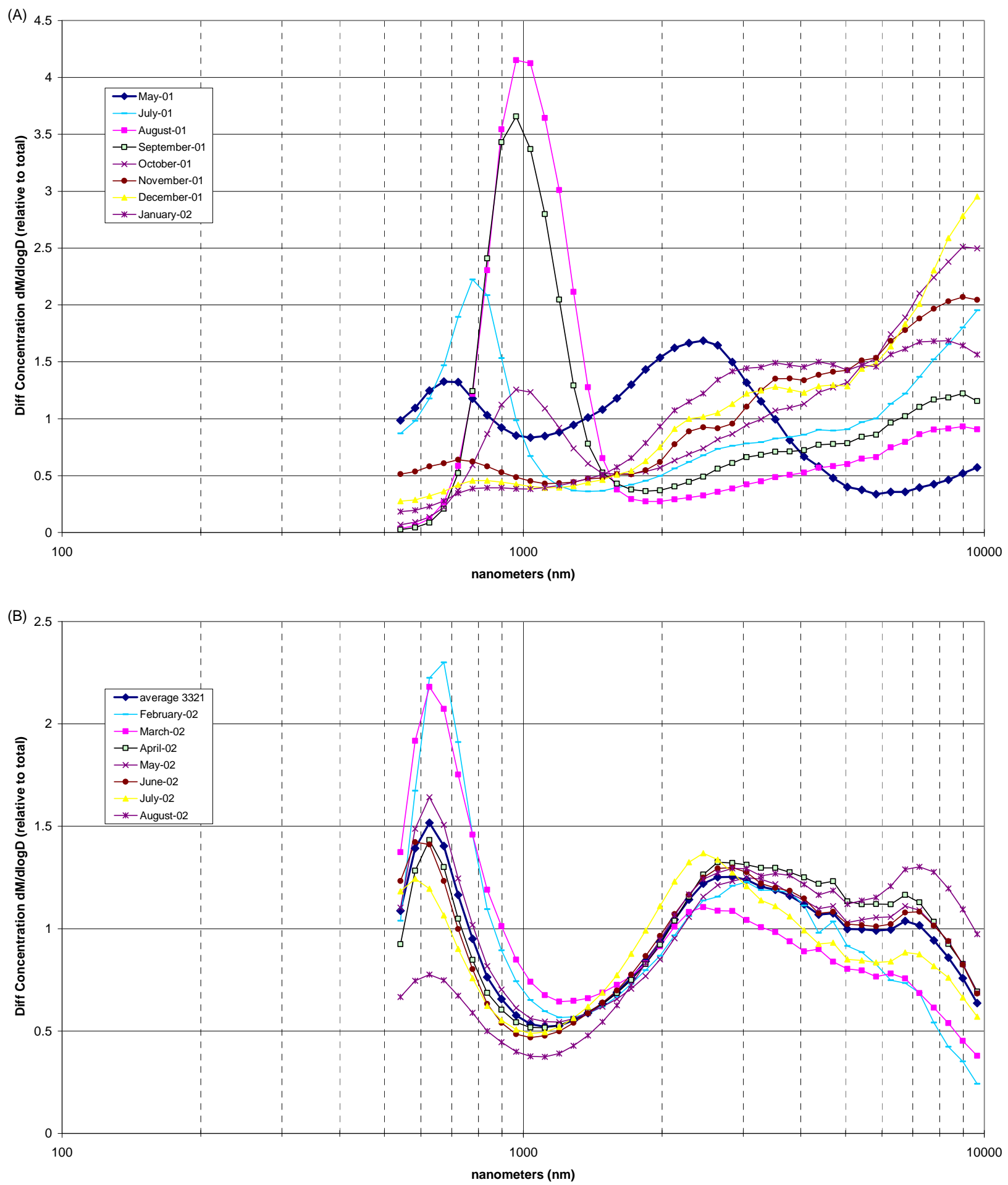

Figure 67. Normalized monthly differential mass weighted particle size distribution for APS instruments: (A) APS 3320, (B) upgraded APS 3321. 


\section{Particle light scattering coefficient: Nephelometer Data}

Figure 68 plots hourly average Nephelometer Bsc measurements during the project period. The figure also shows the JCHD PM . $_{2.5} \mathrm{FRM}$ data from AIRS, and the ratio of the two measurements. For clarity, the Bsc averages are plotted in units of $10^{-6} \mathrm{~m}^{-1}$, which scales on the same order of magnitude as the $\mu \mathrm{g} / \mathrm{m}^{3}$ TEOM mass concentration units, so the ratios have an unstated multiplier of $10^{-6} \mathrm{~m}^{-1}\left(\mu \mathrm{g} / \mathrm{m}^{3}\right)^{-1}$. During the first summer period the ratio of monitor outputs clusters rises above 2.5, then decreases to 1 during the winter season and rises the second summer, with considerable short-term scatter around what appears to be a seasonal component. These results are consistent with the known humidification behavior of ambient particles. Under conditions of higher relative humidity, particle- bound water will increase the overall particle size and scattering coefficient of the sampled aerosol, leading to higher relative scattering signal relative to the dry particles in the TEOM sample (in this case at $30^{\circ} \mathrm{C}$ ). This humidification effect should be greater in the warmer months when atmospheric moisture levels are higher. A scatter plot of the daily average Bsc against the contemporary FRM or TEOM average is contained in Figure 69. Alternately a nonlinear (e.g., quadratic) fit could be used to reflect that scattering is not strictly linear with particle mass when the particle size or composition also change.

$\underline{\mathrm{PM}}_{2.5}$ sulfate: R\&P 8400S sulfate monitor data

Figure 70 plots daily average $\mathrm{PM}_{2.5}$ sulfate mass concentration measurements during the project period as measured by the $8400 \mathrm{~S}$ ambient sulfate monitor along with data from 24-hr filter measurements from the ARA particle composition monitor (PCM) system, and the ratio of the two sulfate measurements. Also plotted on the figure is the ratio of the $8400 \mathrm{~S}$ averages to the mass concentration from the SRI PM 2.5 TEOM; for clarity, only the ratios and not the TEOM mass concentrations are plotted on this figure.

The $8400 \mathrm{~S} / \mathrm{PCM}$ ratio is scattered around a value near 1.0, as expected, but the scatter in the daily averages is more than is desirable and the fit suggests an offset of approximately $1 \mu \mathrm{g} / \mathrm{m}^{3}$. The comparison data is somewhat sparse due to the third- day PCM schedule coupled with the loss of some 8400 days with fewer than 21 valid hourly averages. The TEOM ratio scatters around a sulfate to total mass ratio of $21 \%$ for the overall monitoring period; with a seasonal trend to higher relative sulfate in the summer months. These instrument comparisons are shown more clearly on the scatter plots of Figures 71 and 72 below. 


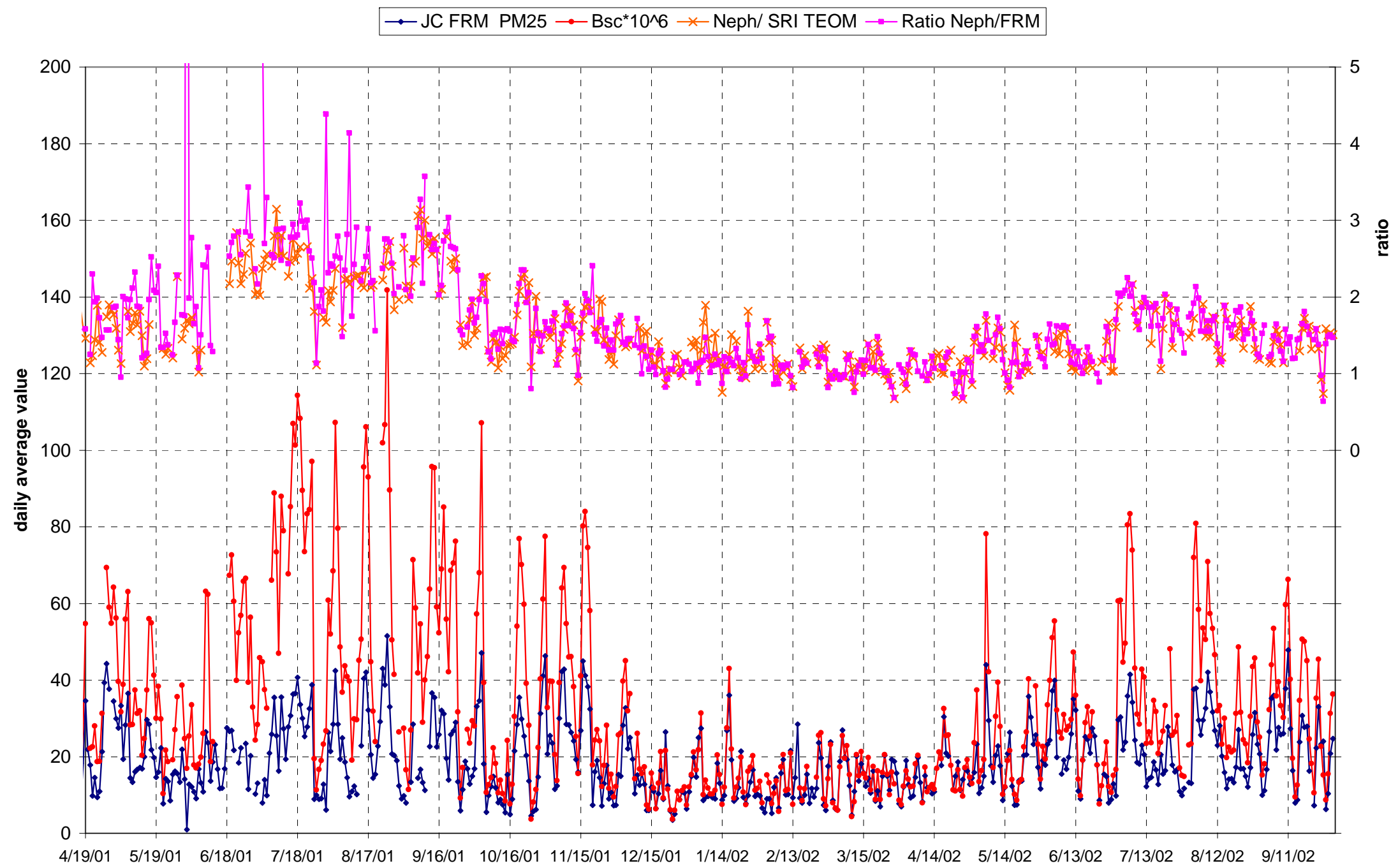

Figure 68. Daily average Nephelometer Bsc values compared to $\mathbf{P M}_{2.5}$ mass concentration from JCHD FRM. Ratios of Nephelometer Bsc averages to PM$_{2.5}$ from FRM and SRI TEOM are shown on second scale. 


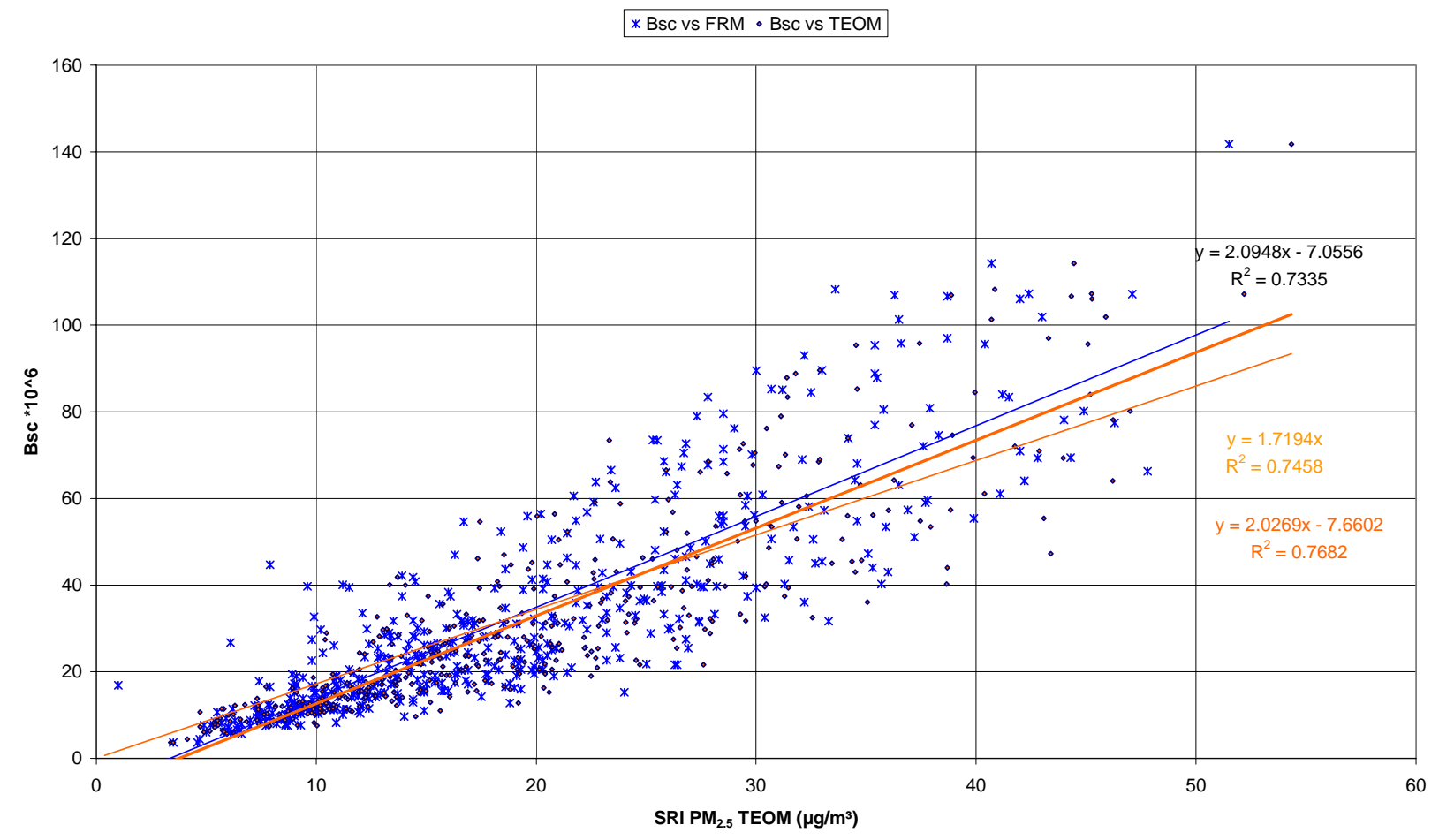

Figure 69. Scatter plot of daily average Nephelometer Bsc values compared to SRI TEOM and FRM PM 2.5 mass concentration. 


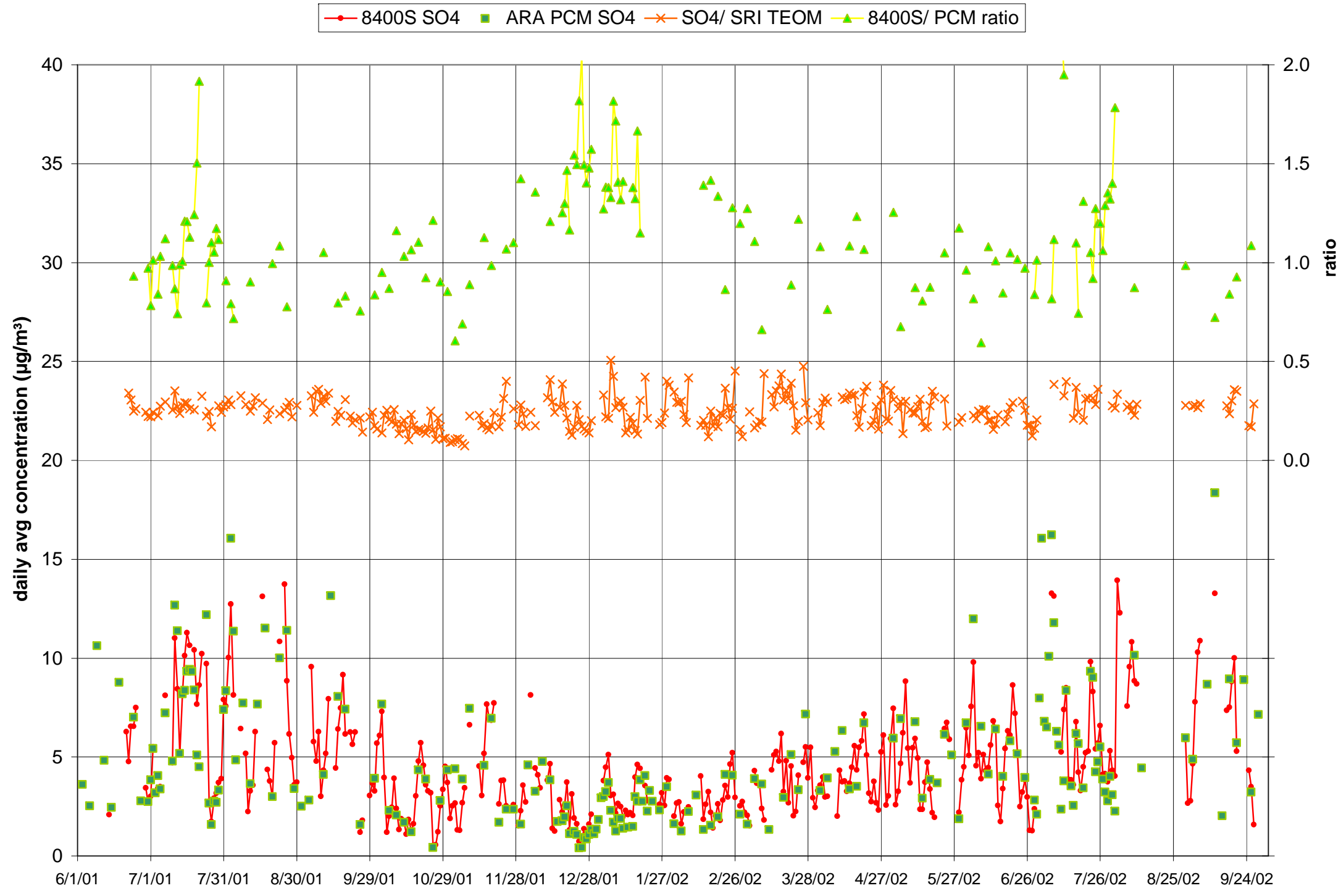

Figure 70. Daily average particle sulfate mass concentration measurements by SRI 8400S monitor compared to ARA PCM sulfate and SRI TEOM. Ratios of 8400S sulfate to PCM sulfate and to TEOM mass are shown on second scale. 


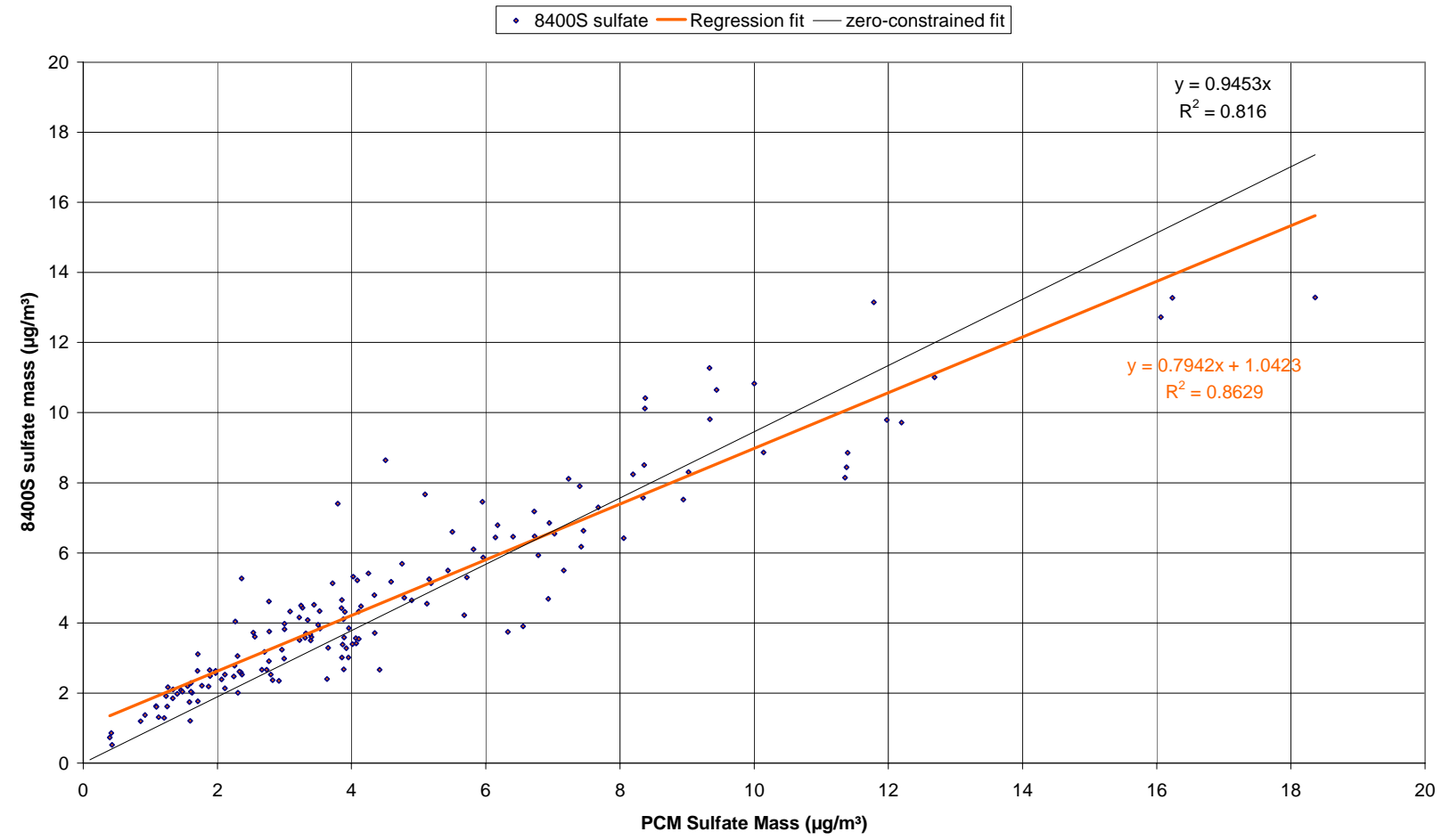

Figure 71. Scatter plot of daily average 8400 S sulfate mass concentration compared to ARA PCM sulfate over project period.

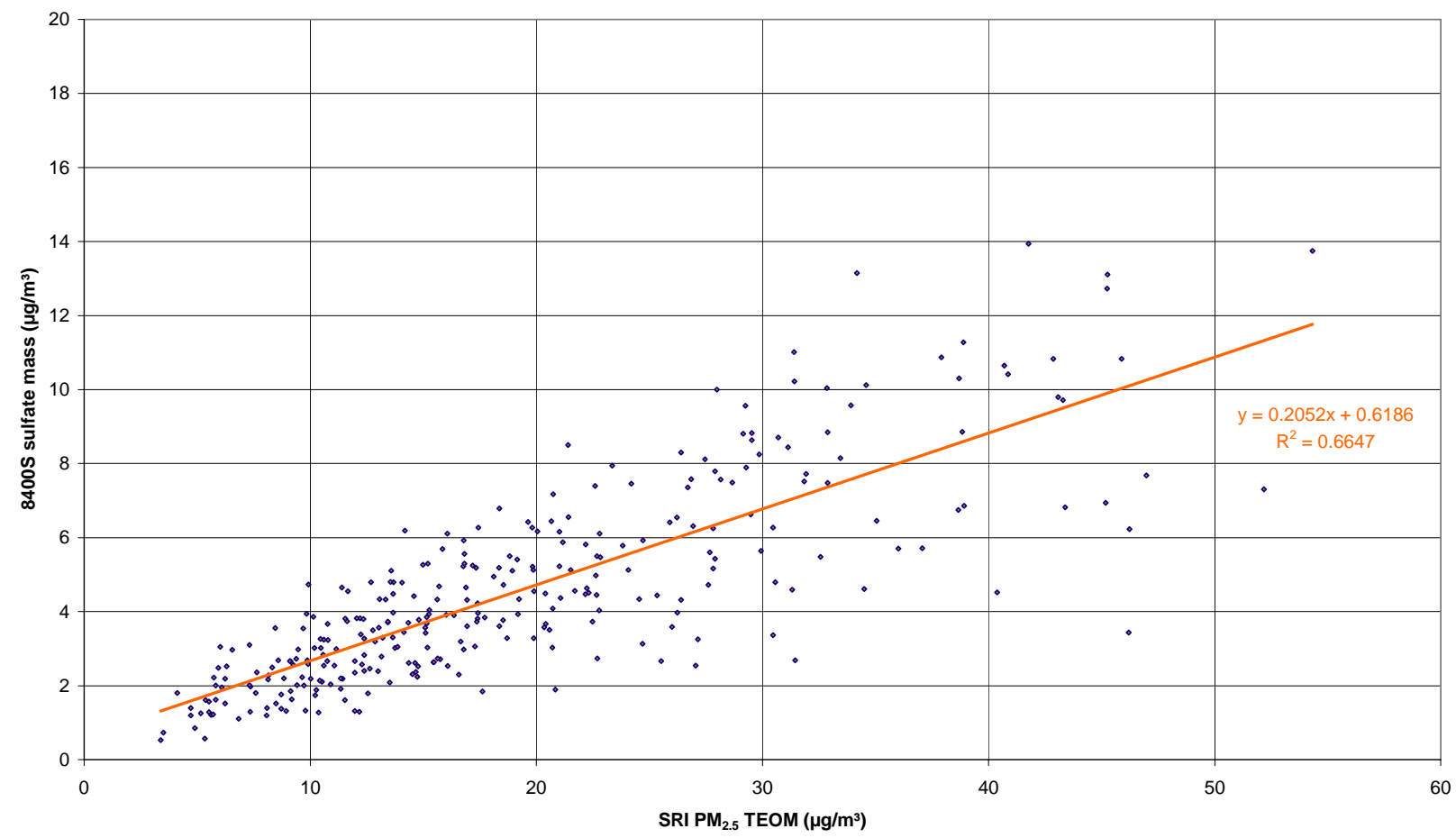

Figure 72. Scatter plot of daily average $8400 \mathrm{~S}$ sulfate mass concentration compared to SRI PM P.5 $_{2.5}$ TEOM over project period. 


\section{Application to Source Attribution, PM Transport, and $\mathbf{P M}_{2.5}$ Management Strategy}

During the course of the project several data analysis and modeling studies were conducted to test the potential of the North Birmingham site data for source attribution analyses. As described in the introductory section of this report, a key aspect of this site is its central location relative to several important classes of local and midrange emission sources. These were marked on the maps in Figures 2 and 3 of that section. As listed there, midrange sources include the three major coal-fired utility stations in the vicinity of Birmingham, (located at distances and bearings, respectively, of 24, 39, and $48 \mathrm{~km}$, and 292, 263, and 136 degrees, relative to North), and a cluster of sources to the southwest of the site associated with the iron and steel industry, including a coke plant, steel plant, and steel pipe mill. Key local industrial emission sources include three directional clusters of sources. Roughly in a line to the Northeast (bearing about $45^{\circ}$ ) are two coking plants, a mineral fiber plant, and a cast iron pipe foundry. To the Southeast (bearing from $115-135^{\circ}$ ), another mineral fiber plant and two iron and steel sources are located within $3 \mathrm{~km}$ of the site, roughly in line with one of the coal-fired power plants. Toward the Southwest (bearing from $230-245^{\circ}$ ) is a cast iron pipe mill 2-km distant, as well as are the more distant iron and steel industry sources described above. No significant local sources lie in the West to Northwest quadrant containing the remaining two coal-fired power plants.

Further discrimination of source classes in these directional clusters is possible using the available compositional information from the continuous gas and particulate monitors. The simultaneous sulfate and $\mathrm{SO}_{2}$ data available form the $\mathrm{R} \& \mathrm{P} 8400 \mathrm{~S}$ are especially useful in this regard, since major sources of $\mathrm{SO}_{\mathrm{x}}$ are relatively limited, and expected to primarily indicate coal combustion or coking. An indication of the potential usefulness of $\mathrm{SO}_{2}$ as a tracer species can be found in the directional data in Figure 73. This figure depicts the distribution of 10 minute average wind direction values (binned in $10^{\circ}$ increments) corresponding to valid $8400 \mathrm{~S}$ measurements during the period from July 2001 to January 2002. The measured wind direction frequency does not show a dominant prevailing direction, though frequencies corresponding to lower wind speeds are enhanced in the $30^{\circ}-240^{\circ}$ directions followed by the local topography. Also plotted is the fraction of measurements for each directional bin having $\mathrm{SO}_{2}$ concentrations greater than $12 \mathrm{ppb}$. This measure has a strong directional signature. The two peaks centered near $300^{\circ}$ and $270^{\circ}$ strongly suggest the two nearest coal fired utility plants; the third plant undoubtedly contributes as well to the peak at $125^{\circ}$, but the height of this peak and of the broad frequency contribution below $90^{\circ}$ suggest that the local industrial sources also contribute strongly to the number of high $\mathrm{SO}_{2}$ events from these easterly directions.

To illustrate the identification of contributions from individual sources using $\mathrm{SO}_{2}$ peaks as tracers, several individual events were surveyed and characterized. Figure 74 shows three such characteristic events during the period July 10-15, 2001. In the figure are plotted the $\mathrm{SO}_{2}$ and particle sulfate channels of the 8400S, corresponding 10 minute averages of the local mean wind direction and of the $\mathrm{PM}_{2.5}$ mass concentration from our TEOM, as well as averages of the CO and NOy* signals from the SEARCH monitors. The first such episode in the figure is the series of $\mathrm{SO}_{2}$ peaks on the morning of July 10 accompanied by significant peaks in reactive nitrogen species but no corresponding increase in $\mathrm{CO}$, $\mathrm{PM}$ sulfate or $\mathrm{PM}_{2.5}$ mass concentration. Trajectory analysis indicates the likely source of $\mathrm{SO}_{2}$ in this air mass to be the nearest coal-fired power plant at $293^{\circ}$. The measured gas and particulate species profile is also consistent with the fresh plume of a typical coal-fired power plant. 


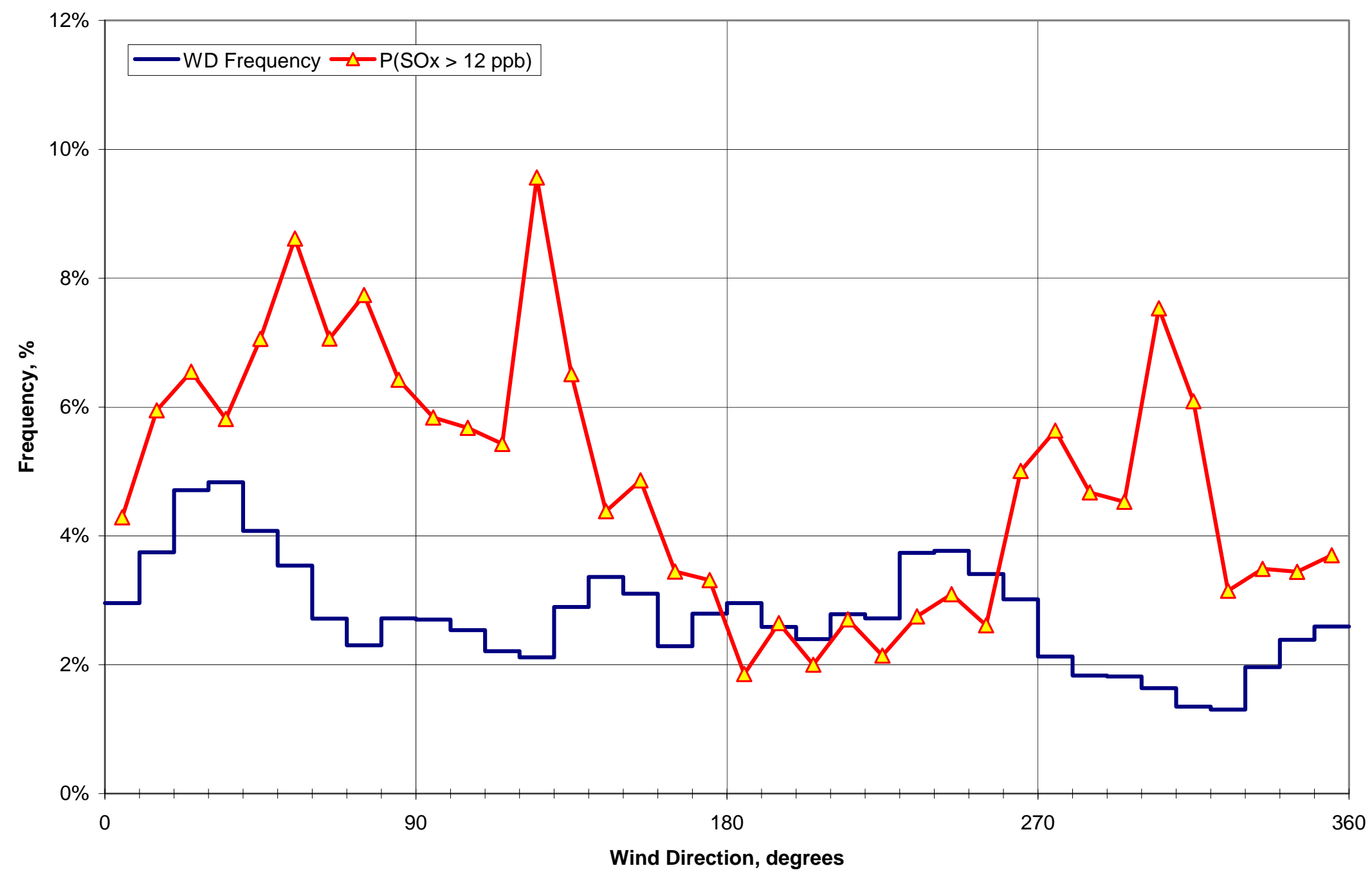

Figure 73. Ten minute average wind direction values (binned in 10 degree increments) corresponding to $8400 \mathrm{~S}$ data from July 2001 through January 2002. 


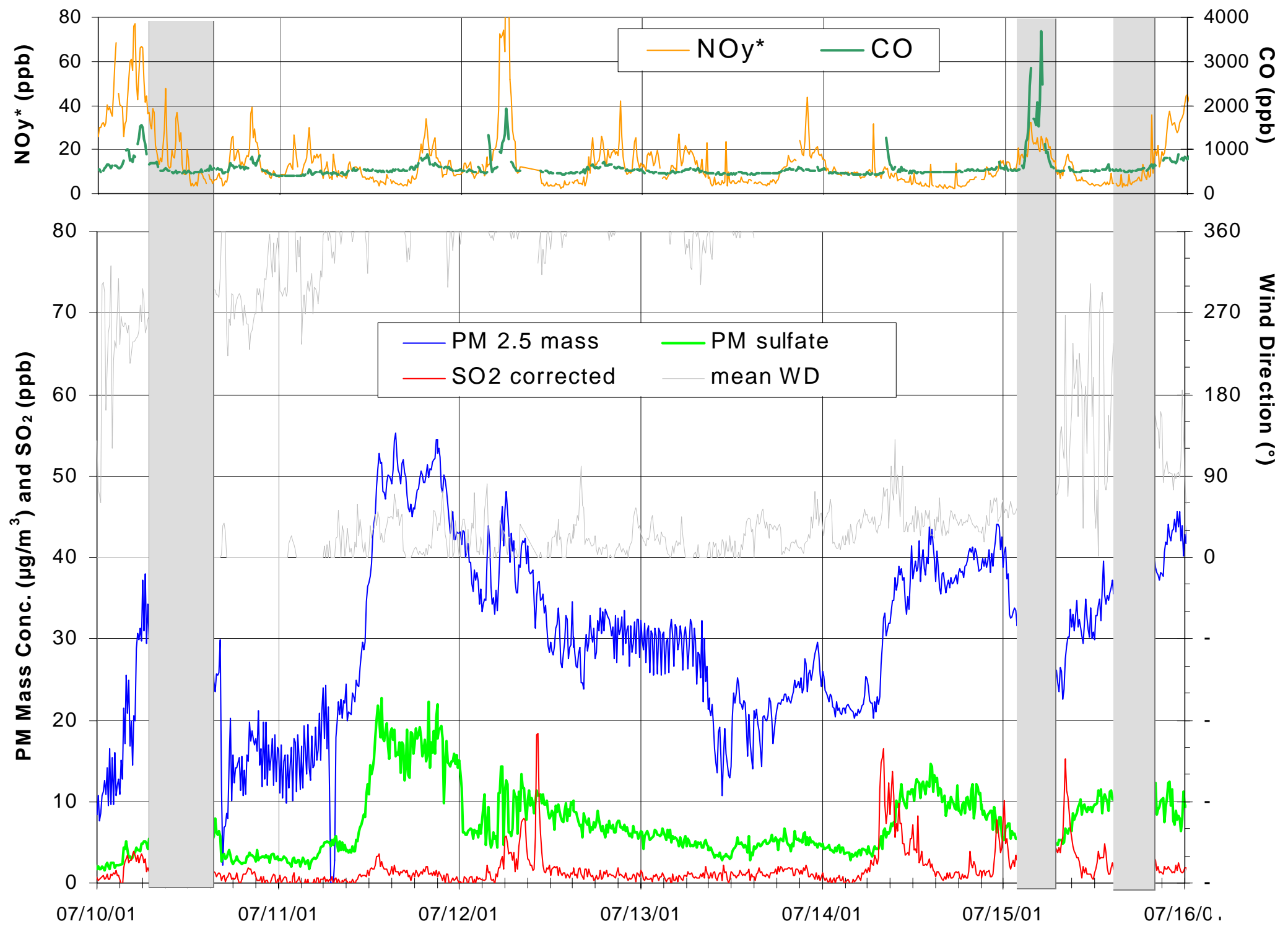

Figure 74. Three individual episodes during July 10 - 16, 2001 analyzed for source identification utility. 
Also highlighted in Figure 74 are two high $\mathrm{SO}_{2}$ episodes on July 15. The first episode occurs during the morning hours, in which a series of $\mathrm{SO}_{2}$ peaks occur. While NOy* signals generally track all these $\mathrm{SO}_{2}$ peaks, other pollutant measures do not, and fluctuate over much of this time period. However, the central $\mathrm{SO}_{2}$ peaks at 3:20 and 4:50 AM (CST) show clearly corresponding peaks in $\mathrm{CO}, \mathrm{PM}$ sulfate and $\mathrm{PM}_{2.5}$ mass concentration, suggesting a distinct, combustion-related source of the $\mathrm{SO}_{2}$ in these time periods. Back trajectories during this time range lie in the quadrant to the northeast of the site, where are located the collection of sources described above, including the two coke plants. It is likely that the measurements currently available at the site can track this source type, even in proximity to other plants of other industrial classes.

The third episode is a relatively isolated $\mathrm{SO}_{2}$ peak between 4:10 and 7:00 $\mathrm{PM}$ on the same day, which occurs without corresponding changes in $\mathrm{NOy}^{*}$ or $\mathrm{CO}$ levels, but is accompanied by corresponding peaks in $\mathrm{PM}_{2.5}$ mass concentration, and specifically in PM sulfate. Integrated concentration measurements of these three species show that the sulfate peak, as sulfate ion, would account for $77 \%$ of the added mass in the $\mathrm{PM}_{2.5}$ mass concentration. This high fraction places stoichiometric constraints on the possible sulfate species involved, since the counterions per sulfate must have total molar weight less than about 30 . Reasonable candidates are sulfuric acid, or ammonium bisulfate, or less probably $\mathrm{NaHSO}_{4}$. A candidate fully neutralized sulfate species is $\mathrm{MgSO}_{4}$, but it is not clear that the $8400 \mathrm{~S}$ would detect sulfate from this inorganic salt with reasonable efficiency. The device would, however, respond to sulfur or any condensed phase reduced sulfur compounds if such species were present. As might be anticipated, the SEARCH continuous carbon instruments (R\&P 5400 and aethelometer) show no increase in particulate carbon corresponding to the mass peak. In short, the source origin of the third episode is not completely clear. The air mass over this time period passed through a broad band of the Southeast quadrant before reaching the site, passing near (but not directly over) the nearby cluster of sources; however, the composition of the particulate species does not seem typical for them. We consider this event interesting, but unexplained.

Since our particle sizing instruments were operating during the last two of these episodes, we were able to investigate changes in the particle size distribution which may be characteristic of the specific emission sources attributed to the events. Figure 75 shows particle mass concentrations in several size bands over the period covered by Figure 74. Over the entire period, including the two episodes, most of the particulate mass falls in the two submicron size bands of $120-300 \mathrm{~nm}$ and $300-500 \mathrm{~nm}$. The smaller $(120-300 \mathrm{~nm})$ of these size fractions dominates in the morning episode, and to a lesser extent, in the background period before and afterwards. In contrast, the 300-500nm fraction is comparable in magnitude during the afternoon event, and slightly greater in the background period before and after this episode. (Similar shifts in the size fraction can be seen in the earlier broad episode of high particulate levels from July 11-13). In Figure 15 the SMPS size distribution for the two July 15 episodes is shown in more detail. During the morning episode (event 1) a broad background size distribution peaked at roughly 60 $\mathrm{nm}$ (number basis) is supplemented by a similarly broad distribution from the event. Given the low ventilation during this time frame, both distributions should be dominated by relatively fresh particles of local origin. The net event enhancement of the size distribution calculated from background subtraction is peaked near $80 \mathrm{~nm}$, and appears to be sparse above $300 \mathrm{~nm}$. The afternoon episode (event 2) shows a more clear contrast between the aged urban/regional background aerosol predominantly larger than $200 \mathrm{~nm}$ and a narrower event size distribution peaked around $120 \mathrm{~nm}$. The figure shows a mode at larger particle diameter for the second event and not the first; however, these features are less certain due to the uncertainty in the changing 
background near the events, complicated by an intervening period of instrument outage. In any case, the figure demonstrates that event particle size distributions may be determined for favorable cases, will have some utility in characterizing the primary particulate from local sources, and add a useful variable to assist in source attribution receptor analysis. 


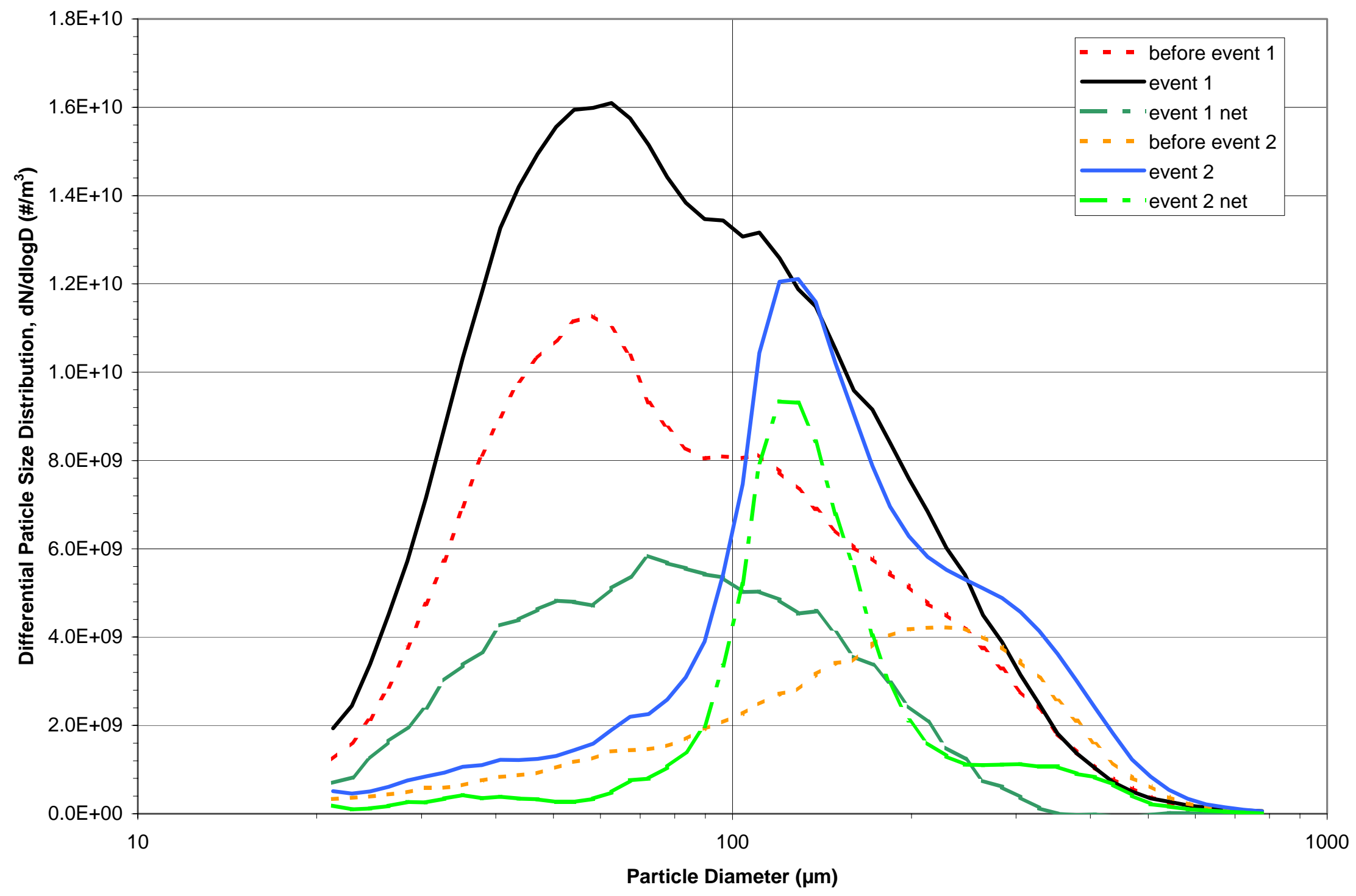

Figure 75. SMPS differential number size distributions for two individual events on July 15, 2001 


\section{Conclusions}

This report is the fruition of a two year investigation involving eighteen months of actual onsite monitoring of ambient fine particulate matter in the Birmingham, AL metropolitan area. As summarized below, all project objectives were addressed successfully. This project involved installation and use of a suite of particulate monitoring instruments, some of which were not in common use at the beginning of the project period. These measurements augmented and complemented existing measurements at the site under the Jefferson County Health Department and the SEARCH program, to the mutual benefit of all. Specific redundant and parallel measurements allowed method intercomparison and validation for key measurements, and the expanded data set has proved useful in characterizing time/transport properties, source attribution, and evaluation of PM management strategies.

Measurement method intercomparison data were developed for the following instruments or measurement quantities:

- $\mathrm{PM}_{2.5}$ mass concentration by $30^{\circ} \mathrm{C}$ TEOM/SES drier vs. $50^{\circ} \mathrm{C}$ TEOM vs. FRM

- Particle size distribution and fractions vs. mass concentration samplers

- Particulate sulfate by R\&P 8400S monitor vs. SEARCH PCM, EPA Speciation Monitor, and HSPH/SEARCH semicontinuous sulfate monitor

- Bscat by Nephelometer/drier vs. "smart heater" controller

Several relevant findings of this project regarding local fine particulate are summarized below.

The North Birmingham site experiences ambient $\mathrm{PM}_{2.5}$ concentrations in excess of the Ambient Air Quality Standard. While no day in our study exceeded the $65 \mu \mathrm{g} / \mathrm{m}^{3}$ daily average standard for $\mathrm{PM}_{2.5}, 60 \%$ of the days exceeded the $15 \mu \mathrm{g} / \mathrm{m}^{3}$ annual standard, $39 \%$ were over $20 \mu \mathrm{g} / \mathrm{m}^{3}$, and $15 \%$ over $30 \mu \mathrm{g} / \mathrm{m}^{3}$, resulting in a (non-calendar year) twelve month average of $18.5 \mu \mathrm{g} / \mathrm{m}^{3}$, consistent with the standard exceedences every year since the beginning of the project.

$\mathrm{PM}_{2.5}$ concentrations at the site follow a seasonal time course, with elevated levels in the summer months and lower concentrations in the December - February periods. The short-term time course of the $\mathrm{PM}_{2.5}$ concentrations likewise has characteristic summer and winter patterns, related to the seasonal meterological patterns. The summer period is characterized by recurring multiday periods having elevated $\mathrm{PM}_{2.5}$ in conjunction with lower mean daily wind speed and very low overnight wind speed. These occur most notably as clear, sunny, stagnant periods under the influence of stable high pressure cells which also lead to episodes of elevated ambient ozone. During these periods particulate levels follow a prototypical daily pattern, with a characteristic morning peak shortly after sunrise, falling quickly to fairly stable low levels during the daylight hours, then rising gradually to elevated levels overnight, then rapidly into the next morning peak. Winter multiday episodes are driven by a different regional weather pattern: they are less frequent, shorter in duration, and generally lower in concentration. While the day/night pattern is always evident, the morning peak behavior is less regular and may be absent.

During the stagnant episode periods with highest particulate concentrations, the impact of local sources is proportionally very high. This effect is seen in several study results, including: 
- a significant weekday/weekend difference, which varies with pollutant and particle size range. The greatest variation is seen in $\mathrm{PMc}$, NO and NOy , and the ultrafine particle size range $(<100 \mathrm{~nm})$.

- The morning peak is reduced in size on weekends as source emissions decrease. For the most reactive species above, the peak disappears entirely on Sunday mornings.

- recurring patterns of submicron particle size distribution, as a stable aged urban/regional aerosol with peak particle size around 200nm mixes with a changing amounts of fresh locally generated aerosol. The local particle mix is numerically dominated by a particle mode around 50nm typical peak diameter, but contains frequent bursts of micron-range and coarse particles in local source plumes.

$\mathrm{SO}_{2}$ has a different time pattern from the other pollutants, and is less in sync with daily atmospheric mixing patterns. It is apparently much more affected by local source emissions patterns, and accordingly is vary useful as a tracer for these sources, which include several nearby industrial sources as well as the coal-fired utilities near the metropolitan area.

Other sources can be identified by combustion tracers (CO, NO/NOy) as well as characteristic particle size distributions.

Source attribution will likely be an important part of developing management strategy for $\mathrm{PM}_{2.5}$. This is especially true of the North Birmingham site, which among the local monitoring stations has the highest average concentrations. The site is clearly impacted by nearby sources, but the highest source contributions occur during stagnant periods and are not directly tied to the responsible sources by measured wind directions. 